A NEW MICROVASCULAR SLEEVE ANASTOMOSIS

THESIS

for the Degree of

\title{
MASTER OF SURGERY
}

submitted to the University of Cape Town by

Francois J. Duminy M.B., Ch.B (Cape Town) 
The copyright of this thesis vests in the author. No quotation from it or information derived from it is to be published without full acknowledgement of the source. The thesis is to be used for private study or noncommercial research purposes only.

Published by the University of Cape Town (UCT) in terms of the non-exclusive license granted to UCT by the author. 
MT 617.413 DUM $90 \mid 37$. 
for

Ingrid, Zeanne \& Cara 


\section{INDEX}

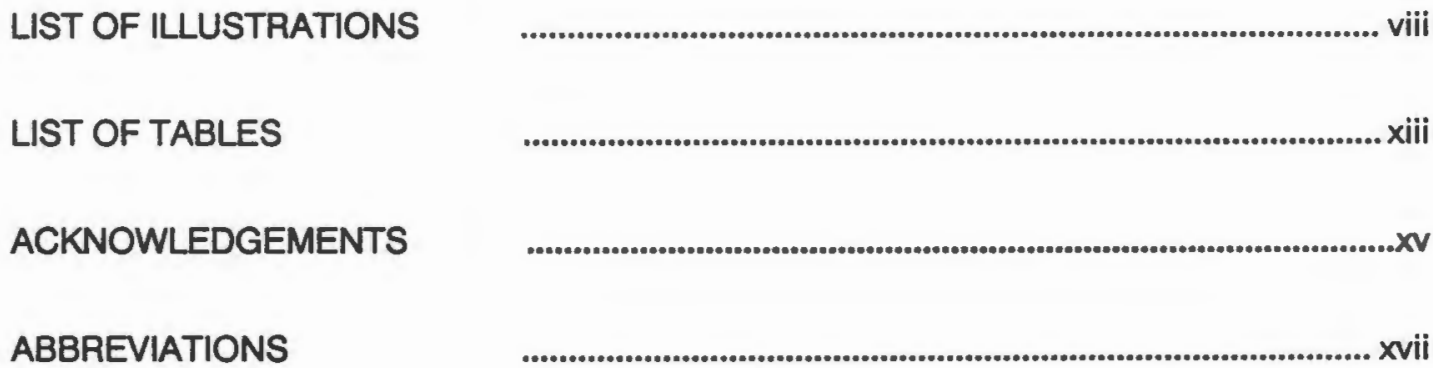

CHAPTER I

INTRODUCTION \& HISTORICAL REVIEW

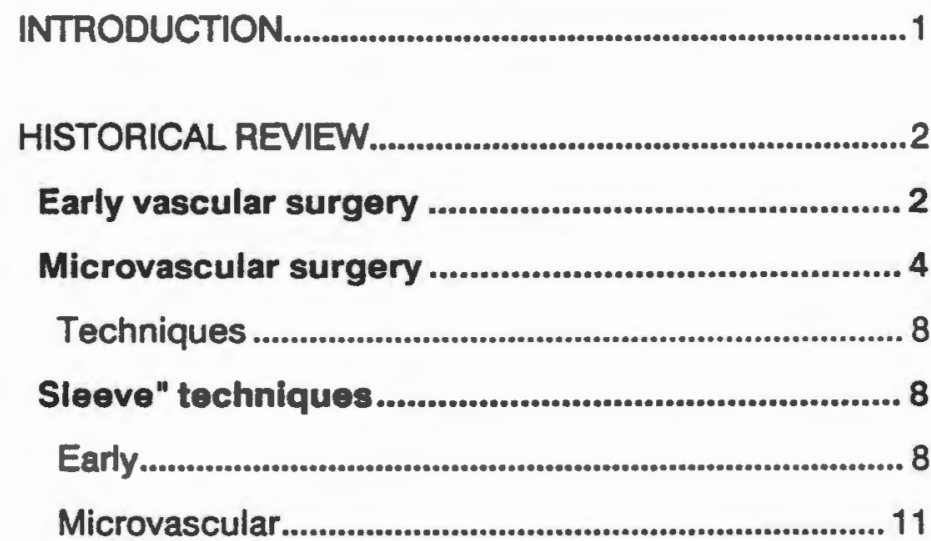

CHAPTER II

QUESTIONS \& OVERVIEW OF APPROACH

PREVIOUS EIEA TECHNIQUES ...................................15

Question 1 .............................................................. 16

Question 2 ............................................................ 18 
Index

THE BASIC QUESTION.....................................................22

Advantages of the EIEA .......................................... 22

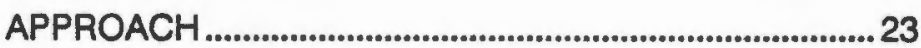

CHAPTER III

THE NEW ANASTOMOSIS

EXAMINATION OF EXISTING TECHNIQUES .................27

The Lauritzen Technique ....................................... 28

The Meyer Technique ............................................. 34

The Hyland Technique .............................................. 38

ANALYSIS OF RESULTS................................................... 41

DESIGN OF NEW TECHNIQUE..................................... 42

Approach............................................................. 42

Problem-Orientated Planning ................................ 44

Jump-outs ......................................................................... 44

Invagination ........................................................................... 44

Entwinement................................................................... 50

Backwall pick-up.............................................................. 52

Extra sutures.................................................................... qq

Summary........................................................................... 55

The Asymmetrical Suture Concept........................ 56

CHAPTER IV. SURGICAL TECHNIQUES FOR THE NEW ANASTOMOSIS

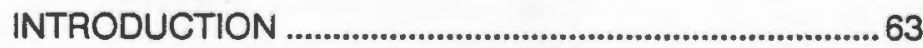

ANASTOMOSIS OF STANDARD MICRO-ARTERIES.....63

Preparation of the vessels..................................... 64

Dissection .......................................................................... 64 
Background material ....................................................66 65

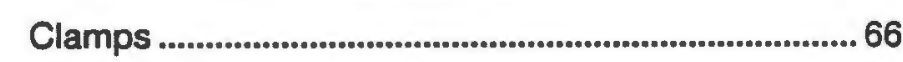

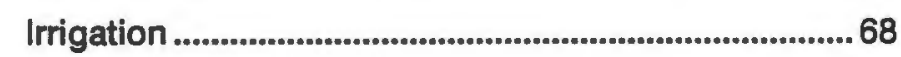

Debridement.........................................................................69

Dilation .................................................................................. 72

Placing the sutures .................................................. 73

Invagination .............................................................. 79

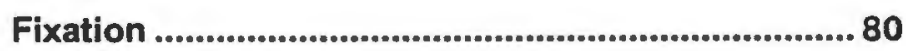

Adventitial advancement ........................................ 81

Preparation for clamp

release .................................................................. 82

Clamp release ............................................................. 85

IMMEDIATE ASSESSMENT OF ANASTOMOSIS ............86

Patency ................................................................ 86

Intraluminal Occlusion ................................................87 87

Extramural Obstruction ................................................. 87

Intramural Obstruction .................................................87 87

Downstream spasm. ....................................................... 88

Intimal dissection ..........................................................88

Haematoma ..................................................................... 88

Intermural Obstruction ................................................... 88

Adequacy.............................................................. 90

Leaking...........................................................................90

Stenosis ....................................................................... 90

Distortion ....................................................................91

MODIFICATIONS FOR OTHER VESSELS ......................93

Standard microveins $(1-2 \mathrm{~mm})$............................. 93

Very small vessels $(<0.4 \mathrm{~mm})$............................... 93

Small vessels $(2-3 \mathrm{~mm})$........................................ 94

Vascular interposition grafts ................................. 94

Autologous vein............................................................. 94

Synthetic material............................................................99

Vessels of dissimilar sizes....................................96 
Index

Arteriovenous shunts . .......................................... 97

Non-vascular tubular structures .............................99

INTRODUCTION

THE EIEA IN DIFFERENT VESSEL SIZES \& TYPES.... 100

INITIAL TESTING IN RAT FEMORAL ARTERIES...........100

Aims........................................................................ 100

Methods and materials ....................................... 100

Results ................................................................... 101

Discussion............................................................ 102

Summary ................................................................ 103

STANDARD MICROVESSELS......................................103

Aims............................................................................. 103

Methods and materials ........................................ 103

Results .................................................................... 104

Discussion................................................................... 107

Summary ............................................................. 108

VERY SMALL VESSELS (<0.4 MM) ..............................109

Aims.............................................................. 109

Methods and materials ........................................ 109

Results ................................................................... 111

Discussion ................................................................ 114

Summary ...........................................................116

LARGER VESSELS (2-3MM)

Aims............................................................................ 117

Methods and materials ......................................... 117

Results ................................................................. 118 
Discussion ............................................................. 120

Summary................................................................. 120

PRIMATE VESSELS ......................................................120

Aims ......................................................................... 120

Methods and materials ........................................ 121

Results.................................................................. 121

Discussion .............................................................. 122

Summary................................................................. 122

VENOUS ANASTOMOSES..........................................123

Aims ................................................................... 123

Methods and materials ......................................... 123

Results......................................................................... 124

Discussion .......................................................... 126

Summary............................................................... 126

UNUSUAL VESSELS - RAT PORTAL VEIN ...................127

Aims ................................................................. 127

Methods and materials ...................................... 127

Results................................................................. 128

Discussion ......................................................... 130

Summary................................................................. 130

CHAPTER VI. COMPARATIVE STUDIES

EIEA VS ETEA IN RAT FEMORAL ARTERY .................133

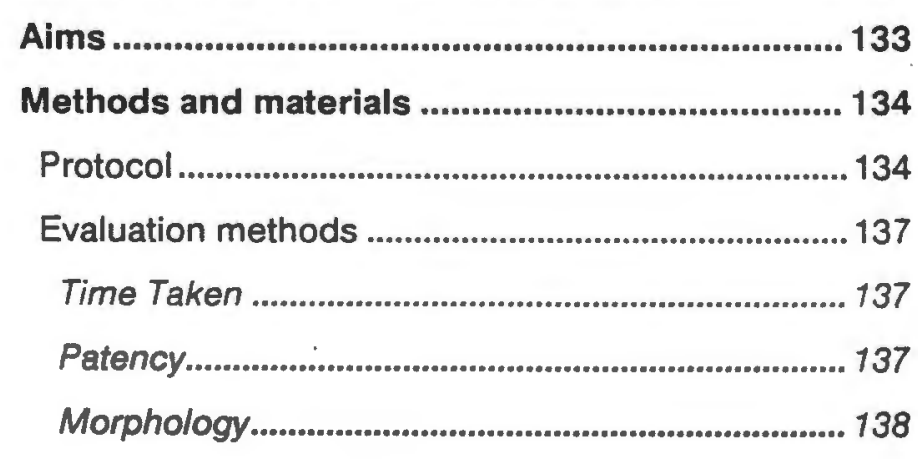


Index

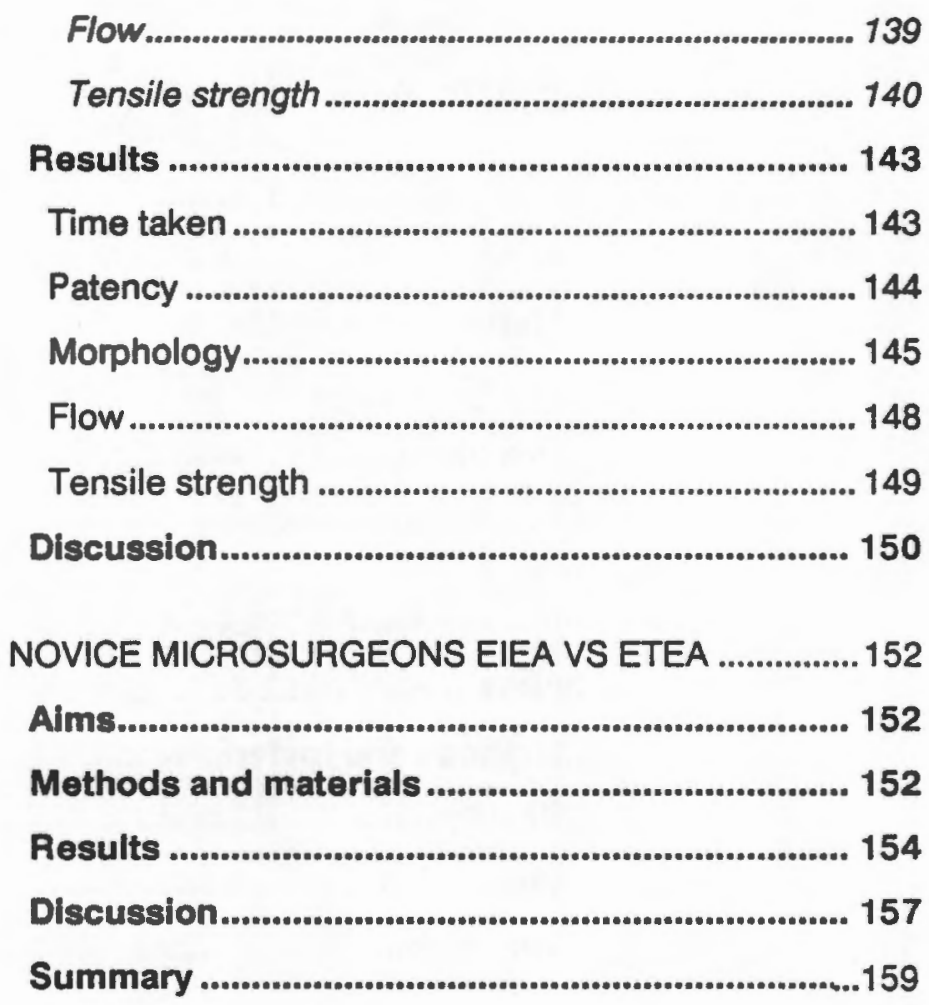

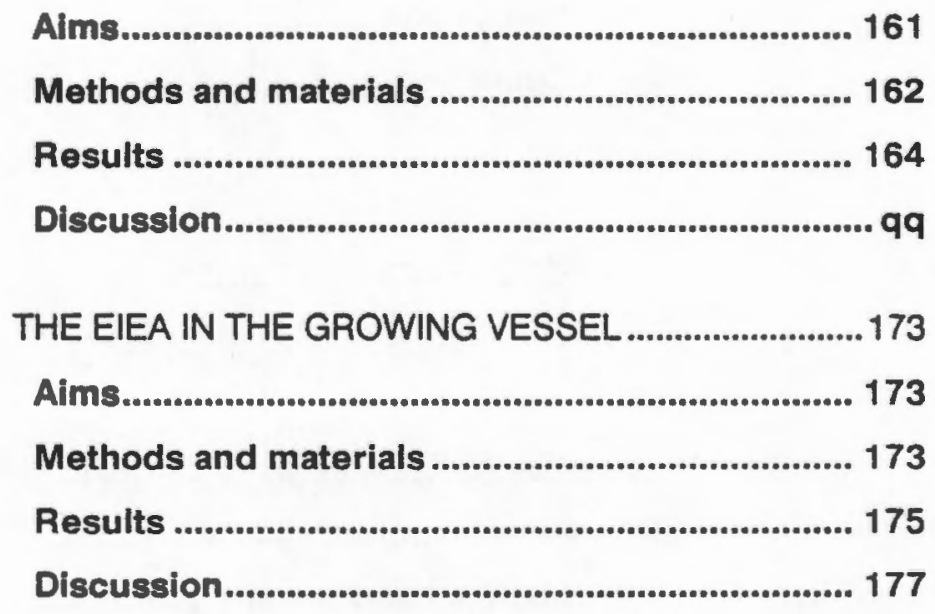


Intimal damage \& exposure of sutures to blood stream in small vessels. 180

Intussusception 181

MECHANICAL ASPECTS ............................................183

Stenosis 183

Flow 183

Shortening. 183

Tensile strength 184

BIOLOGICAL ASPECTS 184

Hoaling 184

Aneurysms. 185

Vessel growth 185

SURGICAL ASPECTS 185

General .186

Timesaving 186

Simplicity. 186

Clinical microsurgery 186

Applications 186

Expectations 189

Experimental microsurgery. 190

Applications 190

Expectations 191

FUTURE RESEARCH..................................................191

Vascular surgery ................................................... 191

Larger vessels ....................................................... 191

Assoc. with adhesives .............................................. 191

Immediate removal of sutures 
Index

Smaller vessels
"Mini-Micro" .............................................................. 193
Lymphatic surgery................................................. 193
CONCLUSION............................................................. 194

APPENDIX A

THE POLISHED-NEEDLE CANNULA

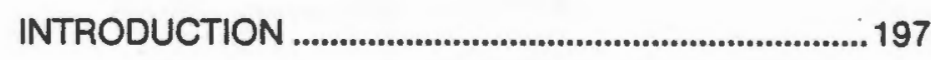

MATERIALS AND METHODS........................................199

Polishing method..................................................... 200

USING THE CANNULA..............................................

Irrigation ................................................................. 203

Counterpressure \& Backwall Protection .............qqq

Hydrodilation ............................................................. 205

DISCUSSION...............................................................206

APPENDIX B THE PRODUCTION AND USE OF CORROSION CASTS FOR

THE EVALUATION OF EXPERIMENTAL MICROVASCULAR ANASTOMOSES

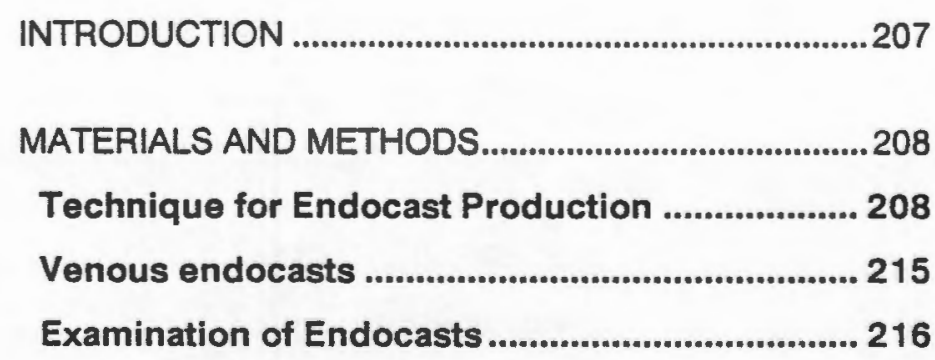


RESULTS ................................................................219

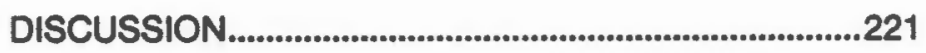

SUMMARY ..........................................................................222

APPENDIX C THE CORROSION CAST EVALUATION OF EXPERIMENTAL MICROVASCULAR ANASTOMOSES IN RAT FEMORAL ARTERIES .......................

APPENDIX D

BIBLIOGRAPHY 
Index 


\section{ILLUSTRATIONS}

Fig. 1.1

Fig. 1.2

Fig. 1.3

Fig. 2.1

Fig. 2.2

Fig. 3.1

Fig. 3.2

Fig. 3.3

Fig. 3.5

Fig. 3.6

Fig. 3.8

Fig. $\mathbf{3 . 9}$

Fig. 3.10

Fig. 3.11

Fig. 3.12
The anastomosis of J.B.Murphy .9

The method of Payr .10

The variety of sleeve methods 12

Looped suture detail .20

Entwined loops causing occlusion .21

Obliquely cut EKG paper measurement guide. .28 Lauritzen technique, all steps. .29 Measuring suture-separation angle. .30

"Tenting" of RV due to disparate suture separations .33

The method of Meyer et al. .35

Hyland's method .39

Effect of gripping forceps too hard .43

The suction-cup invaginator. .47

Methods of towline fixation 49

Flat loop (good) vs Twisted loop (bad) 51 
Illustrations

Fig. 3.13

Fig. 3.14

Fig. 3.15

Fig. 3.16

Fig. 3.17

Fig. 3.18

Fig. 3.19

Fig. 4.1

Fig. 4.2

Fig. 4.3

Fig. 4.4

Fig. 4.5

Fig. 4.6

Fig. 4.7
Acland's counterpressure ring device compared

with the polished-needle cannula

The asymmetrical suture concept 56

Showing the movement of the FV's end when the

2nd suture is tied before invagination. . .57

Three different suture configurations capable of providing dynamic counter traction .58

"Drawing board" design of the new EIEA 59

Only one suture on any single transverse section of the vessel. .61

"Skewing" to anastomose vessels of differing diameters. .62

Grasping the unimportant tissue on either side of the proposed tear before applying the tearing force. .65

The vessel stumps are carefully grasped in a double approximating-clamp .66

The correct position for the clamps. .68

The method for removal of the adventitia. .71

Acland's vessel stretching forceps. .73

The BV75-4 needle [Ethicon] and the Dm 211 needle [SSC] . .74

The deep looped-suture is placed first with the polished-needle cannula in attendance. .75 
Fig. 4.8

Fig. 4.9

Fig. 4.10

Fig. 4.11

Fig. 4.12

Fig. 4.13

Fig. 4.14

Fig. 4.15

Fig. 4.16

Fig. 4.17

Fig. 4.18

Fig. $\mathbf{5 . 3}$

Fig. 5.5

Fig. $\mathbf{5 . 9}$

Fig. 5.10

Suturing method for the new sleeve anastomosis. . .76

Suturing method for the new sleeve anastomosis .77

Suturing method for the new sleeve anastomosis 78

Suturing method for the new sleeve anastomosis .80

Suturing method for the new sleeve anastomosis .81

The RV adventitia is advanced over the cut end of the vessel.

Areas of stress may cause puckering or folding of the vessel walls. 84

"Peanut" swab or a sterile cotton bud (pus swab) is rolled over the anastomosis in the opposite direction to that of the blood flow. 89

The stenosis seen in these casts has always been uniformly smooth and streamlined. .91

Causes of distortion in a line of interrupted sutures. .92

Arteriovenous fistula construction using the EIEA 96

Distribution of EIEA operating times. .105

Distribution of EIEA operating times by novice microsurgeons.

Distribution of diameters of mouse femoral arteries which were reconstituted by the new EIEA.

Vessel diameter vs. operating time in mouse femoral arteries. 
Illustrations

Fig. 5.14

The helical shape of the rat's portal vein.

Fig. 5.16

Corrosion cast of the portal vein of the rat following sleeve anastomosis of the vessel.

Fig. 6.1

The vessel was divided between the deep branch and the superficial epigastric artery.

Fig. 6.2

The "posterior wall first" technique described by

Harris, Finseth \& Buncke (76).

Fig. 6.3

The system by which measurements were obtained

from the photographs of corrosion casts.

Fig. 6.4

Preparation of the vessel for tensile- strength

measurement.

Fig.s 6.5

The apparatus used for measurement of tensile

strength of the vessels.

Fig. 6.7

The distributions of operating-time data for the two

different types of anastomoses

Fig. 6.10

Comparative corrosion casts made in the femoral

arteries of a rat which had an EIEA in one limb [A]

and an ETEA in the opposite limb [B]

Fig. 6.11

A pair of vessels in which the typical mild fusiform

stenosis of the sleeve anastomosis and a large

aneurysm of the ETEA vessel can be seen.

Fig. 6.13

The flow rates in 12 pairs of vessels with

postoperative periods ranging from 1 to 32 days.

Fig. 6.14

Tensile strength in rat femoral arteries. 150 
Fig. 6.18

Fig. 7.1

Fig. 7.2

Fig. 7.3

Fig. 7.4

Fig. 7.5

Fig. 7.6

Fig. 7.7

Fig. 7.8

Fig. 7.9

Fig. 7.10
EIEA time vs. ETEA time showing correlation due to variable skill factors.

Anatomical preparation for obtaining vascular

specimens for SEM. 163

The special container for freeze-drying specimens. .164

The sleeve anastomosis at 3 hours, SEM study .166

The step between the endothelial surfaces of the FV

and the RV is filled with cellular and particulate deposit.

After 8 days endothelial cells have grown over clot in the step area. The endothelial lining is almost complete in this area.

The endothelial surface overlying the step area at

28 days.

A SEM photomicrograph of a corrosion cast of a sleeve anastomosis in the rat femoral artery at 68 days post operation.

The contralateral vessel of the same animal (Fig 7.7)

was anastomosed by means of an ETEA.

Vessels were anastomosed in animals weighing $150 \mathrm{~g}$ and harvested at $450 \mathrm{~g}$ body mass.

The femoral arteries of Long-Evans rats weighing $150 \mathrm{~g}[\mathrm{~A}]$ and $450 \mathrm{~g}[\mathrm{~B}]$ 
FG. 8.1

FG. 8.2

FG. 8.3

FIG. A.1

FG. A.2

FIG. A.3

FIG. A.4

FIG. A.5

FIG. A.6
The "step" in the sleeve anastomosis compared to

that which may occur in the ETEA.

Extracts from medical report on Case 1.

Proposed oblique cut of FV end for largar

vessels. 192

A hand-blunted needle [A] and a commercially

available blunt needle [B] 198

The set of polished needles. 199

The two-pronged fork which results from grinding

too much away. 200

Steps of the polishing process. 201

Introducing the polished needle cannula into the vessel to flush out blood. .203

Hydro-dissection by means of the polished needle cannula. 204 
FIG. B.2

FIG. B.3

FIG. B.4

FIG. B.5

FIG. B.6

FIG. B.7

FIG. B.8
The suture-needle is passed through the myocardium from left to right around the butterfly needle.

The ends of the suture are passed around the needle in opposite directions and tied again on top of the needle and in the drop of glue.

The axially-rotatable stand, specially made for photographing corrosion-cast specimens.

A dark background which was formed by the shadow in a deep hole in the background material 218

Magnified images of the corrosion casts could be used for obtaining accurate measurements.

Measurements were taken from photographic enlargements.

A corrosion cast of a small vessel showing the depressions made by endothelial cell nuclei on the surface of the cast. 
Illustrations 


\section{TABLES}

Fig. 3.4

Fig. 3.7

Fig. 3.8(b)

Fig. $\mathbf{5 . 1}$

Fig. 5.2

Fig. 5.4

Fig. 5.6

Fig. $\mathbf{5 . 7}$

Fig. $\mathbf{5 . 8}$

Fig. 5.11

Fig. 5.12

Fig. 5.13

Fig. 6.8

Fig. 6.9
Lauritzen technique, results. .32

Meyer's method, results .37

Results when using the method of Hyland et al. . .40

Results of new EIEA in rat femoral artery. . .101

Analysis of operating times in 89 EIEAs. .104

Analysis of EIEA operating times by novice microsurgeons. 106

Patency rates for microvascular anastomoses in other published studies. 108

EIEA - Operating time in $1 \mathrm{~mm}$ arteries 109

The EIEA in mouse femoral artery. .111

EIEA in porcine femoral artery. 119

Results of EIEA in Chacma baboon arteries. 122

The EIEA in rat femoral veins. 125

Analysis of comparative operating-time data for

EIEA vs. ETEA. 144

Comparative patency rate of EIEA vs. ETEA 
Tables

Fig. 6.12

Analysis of results obtained from measurements of corrosion casts.

Fig. 6.15

Patency rates compared in vessels anastomosed by novice microsurgeons. 154

Fig. 6.17 Analysis of Novice operating times. 156

Fig. 7.12

Results of measurements obtained from corrosioncasts of vessels which had been anastomosed in the young growing animal. 


\section{ACKNOWLEDGEMENTS}

A number of people and institutions have rendered assistance and support in this work and I wish to thank them for their contributions. My sincere thanks to the following:

Dr J.P. Lowe, Consulting Medical Officer of Goldfields of South Africa, who introduced me to the operating microscope and has done much to support and further my interest in the field of microvascular surgery.

Dr C.C. Freed, visiting consultant to the Leslie Williams Memorial Hospital, who guided my early efforts in recording my work. His encouragement and support lent much impetus to this work.

Professor Harry J. Buncke Jr., Chief of the Transplantation and Replantation Centre of the Ralph K. Davies Medical Centre in San Francisco. It was my great privilege to spend three months in the Microsurgery laboratory of that organisation and it was in that stimulating environment that I developed the basis of the new sleeve anastomosis. The enormous vigour, enthusiasm and enterprising spirit of Professor Buncke make him an example I would wish to emulate in my career. I would also like to thank him and his associates, Doctors Bud Alpert and Leonard Gordon, for their interest and suggestions in the early stages of this research project and for the valuable insight into clinical microsurgery I was allowed to obtain during their ward rounds and in their operating rooms.

Leonard Newlin and Beatrice Vasconez, who were always willing to assist me and who made me feel at home in their laboratory and in the City of San Francisco.

Professor Enrique Zea Longa, Brazilian Neurosurgeon, whose innovative thinking stimulated my use and development of resin corrosion casts for the evaluation of anastomoses.

Dr Austin of the University of the Witwatersrand Animal Laboratories for the assistance he gave me during the part of this research which was carried out at The Leslie Williams Memorial Hospital in Carletonville. Ben Rademeyer and his Laboratory ladies also deserve a special vote of thanks for the excellence of their animal husbandry during that period. 


\section{Acknowledgements}

Dr-Johan Van der Spuy, Consultant in charge of the Trauma Unit, Groote Schuur Hospital, whose encouragement, guidance and logistical support were directly responsible for the completion and presentation of this work at this University. He can hardly be adequately thanked here.

Professor John Terblanche of the Department of Surgery of The University of Cape Town, who opened his research facilities to me and employed me in his department in a position which allowed my research to progress.

Professor Rosemary Hickman, who, with her skills in the planning, execution and presentation of surgical research, has guided much of my passage through this work.

My microsurgical colleague, Gert Engelbrecht for the warm and friendly manner in which I was accepted in his laboratory domain and for the stimulating exchange of technical ideas and innovations arising from his hard-won experience and from our shared enthusiasm for microvascular surgery.

Messrs. Willem Ryneveldt and Nolan Hendricks have won my respect and gratitude by their willingness and ability to supply expert technical assistance in both the mundane peripheral chores and the challenging intricacies of experimental surgery.

Vivien Johns, for frequent administrative troubleshooting and George Graham for ordering the equipment and materials which I required and for making those items which could'nt be ordered.

Phillipa Johnson, Jules Pasacovitch and Sue Abraham for processing, printing and improving the diagrams and photographs without ever complaining despite their enormous workloads.

Dr John Knottenbelt for introducing me to computers and for helping me to experience a faint glimmer of understanding of the most basic statistical methods.

My friends and colleagues, Drs Pieter Pike, John Travlos, Murray Wallace, Patrick Crisp, Duncan Black, Keith Cronwright, Sadick Saban, Phillip Nel, Brndt Sido and Tristan de Chalain who served as "novice microsurgeon" guinea pigs. A special vote of thanks also goes to Tristan de Chalain for introducing me to the wonders of the Scanning Electron Microscope and to Dane Gernicke and his colleagues at the U.C.T. EM Laboratory for their teaching and their ready assistance.

Lisa Duminy who spent much of her valuable time in proofreading and correcting the draft copy. 
This study and thesis would not have been possible without the financial assistance provided by the Goldfields Foundation of Goldfields of South Africa. I am deeply grateful to the Board of Directors of that Foundation for the support that they have given to this work and to the teaching of microsurgery at this university.

Both time and comfort were sacrificed by my wife, Ingrid and my daughters Zeanne and Cara during the pursuit of these studies. They travelled halfway round the globe to be with me, lived in cramped accommodation and moved to new homes twice in the process. I am deeply grateful to them for the love and joy they have continued to provide in spite of difficult circumstances.

I have left until last the most difficult person to thank. My father, J.J.E. Duminy M.Sc B.Ed. (U.C.T), lifelong student and educator, has worked tirelessly in reading, correcting and rereading this thesis innumerable times. To do this he not only travelled long distances every day, but also learned to use a word processor and a computer spreadsheet after his 70th birthday. He has always been, and will always remain more than any man could want in a companion and a father. 
Acknowledgements 


\section{ABBREVIATIONS}

Feeding Vessel

RV

Receiving Vessel

MI 



\title{
CHAPTER I. \\ INTRODUCTION AND HISTORICAL REVIEW
}

\author{
We shall not cease from exploration \\ and the end of all our exploring \\ will be to arrive where we started \\ and know the place for the first time.
}

Thomas Stearns Eliot

\section{INTRODUCTION}

In 1896 J.B. Murphy of Chicago became the first surgeon to successfully anastomose a completely disrupted human artery. He used an anastomotic technique in which the proximal stump of the artery was placed within the distal stump and held there by sutures (135).

In 1978 two surgeons, W.E. Meier of Zurich (131) and Claes Lauritzen of Gothenburg (112), working independently, developed methods which were based on the same principle for effecting microvascular anastomoses.

Lauritzen, who produced a number of publications describing the evaluation and application of his method (107-116), based his support for the method on its technical simplicity and the significant timesaving when compared with other methods.

The term "sleeve" anastomosis, based on its appearance, was coined by Harry J.Buncke Jr., of San Mateo, California ${ }^{1}$, and first used in a publication by Lauritzen.

Since the introduction of this principle to microvascular surgery, a number of workers have used it in experimental and clinical practice $(65,83,87,100,133,142,176,178,179,197,203-207)$. 
Reviewing the work of these researchers and clinicians, I was struck by the diversity of methods which were employed to anastomose microvessels by means of the "sleeve" principle (see Fig. 1.3 ). I fully supported Lauritzen's effort to simplify microvascular anastomoses, but I had certain difficulties in executing the technique he had proposed. It occurred to me that others might have had similar problems and that this might have been responsible for the variety of methods used.

It was against this background that I made it my task to identify the weaknesses and strengths of a number of proposed methods and to use the knowledge and experience thus gained to attempt the development of a new method which would be consistently reliable.

\section{HISTORICAL REVIEW}

\section{EARLY VASCULAR SURGERY}

Before Murphy's time there had been little success in the field of arterial vascular surgery and surgical intervention was limited almost entirely to the ligation of compromised arteries. A notable exception might be that of Cosmas and Damion, saints in Little Asia (circa 300 A.D.), who were reported to have replaced the gangrenous leg of a man with the healthy leg of his recently deceased slave (81). Hess points out that this could only have been achieved by means of some form of vascular surgery!

In the 2nd century A.D. Ruphus of Ephesus and Galen were known to use vascular ligatures, as was Atius of Amida (500-550 A.D.) (81). Antyllus treated aneurysms by. ligating the vessels proximal and distal to them and then opening the aneurysmal sacs. In 1710 Anel placed one ligature proximal to the sac without disturbing it and in 1785 John Hunter advocated placing the ligature well above the aneurysm. His ligature for a popliteal aneurysm was placed in the area which later became known as Hunter's canal (72).

Ligatures, as rediscovered by Pare in the middle of the $16^{\text {th }}$ century $(72,81)$, remained the only method for preventing exsanguination from damaged vessels until Hallowell, in 1759, repaired a damaged vessel by transfixing the edges of the vessel wound with metal pins and then tying a thread around them $(71,161)$. 
In 1762 Broca reported a case in which a longitudinal incision of an artery was successfully repaired by means of sutures $(135,161)$. The first artificial anastomosis between two different vessels was devised by Nikolai Eck in 1877, when he created a portacaval shunt. This technique was lost for many years and was rediscovered after the advent of decompressive surgery for portal hypertension (72).

Jassinowski of Odessa reported important work in the suture repair of vascular lacerations in $1889(135,71,147)$. Heidenhain closed a centimetre-long opening in the axillary artery by means of a catgut suture on 28 May 1894 (135).

Based on the work of these and other surgeons such as Billroth, the closure of wounds in veins by means of sutures had become an accepted surgical procedure at the time of Murphy's arterial anastomosis in 1896 (135).

Briau and Jaboulay reported the successful circumferential suture of a carotid artery in a donkey in 1896 (71). On October $7^{\text {th }}$ of the same year Murphy, as mentioned above, became the first surgeon to successfully repair a human vessel, which had been totally severed, when he reconstituted the femoral artery of a young man who had been shot in the groin. The patient had distal pulses after the operation and made an uneventful recovery (135). The anastomosis he used, was based on invagination of the proximal stump into the distal stump of the artery.

After this success there was considerable interest in the repair of severed arteries and much associated controversy with regard to the need to avoid intimal penetration and the intraluminal placement of suture material. This controversy was "settled" in 1899 by Julius Dorfler, who showed that penetration of the intima did not necessarily induce thrombosis, if sterile silk thread was used (72).

The field of vascular surgery in the first decade of the $20^{\text {th }}$ century was dominated by Alexis Carrel who, with his colleague, Guthrie, laid the foundations of modern vascular surgery $(35,33,31,32,34)$. His work with Guthrie and others, including the aviator Charles Lindbergh, was so wide-ranging, that Sterling Edwards in his preface to his biography of Carrel, wrote: "If you think you've invented a new technique in heart or blood vessel surgery, you'd better check to see if Dr. Alexis Carrel did not try the same thing 50 years ago." (72).

The triangulation (or stay-suture) method of vascular anastomosis, devised by Carrel (35), is used extensively with little modification to this day. 
It is interesting to note that Carrel's initial judgment was that the intima should not be disturbed by sutures $(35,34,31)$, but that he was later persuaded by the work of Dorfler and others to abandon this premise. His examination of the different techniques for vascular anastomosis yielded uniformly successful results with the method of Dorfler and he therefore adopted it from 1905 onwards (71).

Following the Carrel era there was little development in the basic techniques of vascular surgery until the second half of the $20^{\text {th }}$ century. Despite the fact that the intervening world wars might have provided a great need for the expertise of vascular surgeons, most of the vascular injuries were treated by ligation $(93,161,211)$. The work of Crafoord and Nylin in the surgical treatment of congenital coarctation of the aorta (48) appears to have heralded a revival of interest in vascular surgery (72).

Carrel had recognised the need for the anastomosis of very small vessels in transplantation of tissues. His solution to the problem was to dissect the small vessel free to its union with the parent trunk and then to excise a "flange" of the larger vessel surrounding the mouth of the smaller vessel. This flange, or "Carrel patch", could then be anastomosed by "macro" techniques to a hole in the wall of a large vessel in the recipient animal $(31,34)$.

O'Brien writes that Schumacker and Lowenberg reported high patency rates with anastomoses of canine arteries as small as $3.2 \mathrm{~mm}$ in diameter in 1948 (147). Thal et al performed anastomoses in vessels of less than $3 \mathrm{~mm}$ in diameter between the internal mammary and the coronary arteries of dogs in 1956, thus paving the way for today's coronary bypass operations (185). Seidenberg et al developed a technique for the anastomosis of small arteries in 1958. Despite the non-availability of small sutures and the fact that they had not used magnification, they reported that the successful anastomosis of vessels ranging from $1.5 \mathrm{~mm}$ to $4 \mathrm{~mm}$ in diameter became the rule in their practice (169).

\section{MICROVASCULAR SURGERY}

\section{THE PIONEERS}

The microscope was first used in experimental surgery by Carl-Olof Nylen in 1921. In the autumn of that year he used a primitive binocular microscope on a clinical case of chronic otitis and in the next year his chief, Gunnar Holmgren, developed the idea further by introducing a 
Zeiss stereoscopic microscope to otology (143). In 1946 Perrit was the first surgeon to use the microscope in ophthalmic surgery (147).

The introduction of this instrument into the field of vascular surgery by Julius $\mathrm{H}$. Jacobson and Ernesto L. Suarez in 1960 was tantamount to a quantum leap in the anastomosis of small vessels. They wrote:

"A $1 \mathrm{~mm}$. error in suture placement results in thrombosis in a $2 \mathrm{~mm}$. anastomosis but has little significance when one works on large vessels."

Aided by magnification of up to $25 \mathrm{X}$, they performed anastomoses on the carotid arteries of 20 dogs and 6 rabbits and reported a $100 \%$ patency rate up to 4 months after the operations (94). (The vessels averaged 3.2 and $1.4 \mathrm{~mm}$ in diameter respectively). Their success in the laboratory and the subsequent clinical application of their techniques (93) gave rise to an "explosion" in microvascular surgery.

In a paper published in 1963, Jacobson described the instruments which had been miniaturised and the techniques which had been developed for microvascular surgery (93). It appears that a $7 / 0$ silk suture was the smallest available at the time $(93,118)$, but that smaller sutures (" $1.5 \mathrm{ml}$. suture on an ultrafine needle") were in the process of being developed (93).

In 1963 Kleinert and Kasdan reported on the salvage of numerous devascularised parts of the upper extremity by means of small vessel anastomosis aided by magnification. They were not successful with complete replantations, but saved fingers and a thumb which had been deprived of an arterial supply (98).

In 1965 Harry J. Buncke was the first to apply the techniques of microvascular surgery to the field of plastic and reconstructive surgery (147). Together with his wife, Dr. Constance M. Buncke, and W.P. Schultz he performed experiments in the fields of replantation and transplantation of digits and of other tissues, which paved the way for the current clinical successes in these fields $(22,24,23)$. Their vision of the future of microvascular surgery may be summarised by the following excerpts from one of their papers (24):

"With the continued perfection of techniques and instruments these repairs [microvascular] will be performed with ease in a reasonable period of time by any surgeon who is interested in this phase of reconstructive surgery." 
and

"The successful transplantation of a block of composite tissue by reanastomosing the microvescular pedicle has untold experimental and clinical possibilities."

They made another great contribution to the rapid advance of microvascular surgery by developing smaller sutures and needles than any previously available. This they did by metalising the end of a nylon fibre 10 microns in diameter to create a needle-thread combination in which the needle was only slightly thicker than the thread (24).

When the difficulties associated with the anastomosis of small vessels had been overcome by the introduction of the microscope and by the production of delicate suture materials and. instruments, surgeons turned their attention to the clinical applications of their new skills.

In 1906 Carrel and Guthrie wrote:

"If these permanent results are possible, if no trophic troubles occur and if the functions of the transplanted or replanted limb are satisfactory, then it will be legitimate to employ similar operations in human surgery. Of course it is a mere suggestion at present, and further investigation may show that it is quite impossible." (32)

It is therefore interesting that the areas receiving the greatest attention were those of replantation of accidentally amputated structures and the transfer of tissue from one part of the body to another. An obvious prerequisite would be the anastomosis of the vascular pedicle of the tissue to vessels in the vicinity of the "recipient" area.

Krizek et al (105) performed successful experimental free flap transfers in 1965, as did Strauch and Murray in 1967 (177). Cobbett performed the first successful transfer of a great toe to replace an amputated thumb in April 1968 (209). Komatsu and Tamai reported the first successful replantation of : a severed thumb in 1968 (99).

Buncke was the first to report success in a clinical soft-tissue flap transfer operation, when in 1972 he and Capt. Donald McClean transplanted omentum to a $6^{\prime \prime} \times 8^{\prime \prime}$ defect in the scalp of a 29yrold man, anastomosed the omental vessels to the superficial temporal vessels and covered the vascularised transplanted omentum with a meshed split-thickness skingraft taken from the right hip (130). In February 1972 they and their team, like Cobbett, successfully transplanted the great toe of a $30 y$ rold fireman to his dominant right hand to replace the thumb he had lost in a powersaw accident. Six months later the "thumb" had $30^{\circ}$ of flexion in the distal interphalangeal joint and a 
good range of motion at the metacarpo-multangular joint. His grip strength was $80 \%$ of that of his left hand and he had protective sensation to pain, heat and cold at the tip of the digit. Forceful functional pinch had been restored (28).

In the same year Harii and Ohmori et al transferred a number of free vascularised skin flaps and published their results in 1974 (75).

Reconstructive surgeons now fervently sought anatomical areas with well-defined, axial blood supply, which could be used routinely as donor areas for free transfer to other areas on the same individual. The possibility of autologous transfer of tissues from anatomical areas in which their presence was not vital, to others in which they might help to preserve function or viability, was the greatest motivating force for the development of microvascular surgery.

Either Daniel and Taylor (52) or O'Brien et al (146) led the way in 1973, when they independently performed free transfers of the well-known groin flap, which had faithfully served reconstructive surgeons as a pedicle flap. Many other suitable donor sites for flaps were defined and used in the years that followed and the work continues $(75,17,53,25,26,12,84,151)$.

The development of replantation techniques was intimately associated with those of free-flap surgery. In many cases replantation was augmented by simultaneous free-flap transfer (personal observations).

Associated with the transfer of free vascularised tissue flaps and the replantation of amputated body-parts, a need has developed for methods to monitor them. Survival rates could possibly be improved by timeous intervention against impending compromisation of their arterial supply or venous drainage, if adequate forewarning of such events were available $(80,27$ [p.29 \& pp.399424]).

The possibilities for free-flap transfer may be vastly expanded when adequate management of immune responses can be performed safely enough to lend feasibility to the heterogenous, homozygous transplantation of tissues. To this end research efforts are also directed towards the preservation of flaps for extended periods of time (187). 


\section{TECHNIQUES}

The techniques used by Jacobson (93) were a direct transposition or adaptation of the methods developed by Carrel and used in the surgery of large vessels. He used continuous-suture methods for all his vascular anastomoses.

Microvessels were too small for 3 stay-sutures and in 1967 Cobbett (45) suggested an adaptation for microvascular surgery of the method of Smith ${ }^{2}$ wherein the stay-sutures were placed $120^{\circ}$ apart to allow the posterior wall to fall away from the anterior wall. This helped to prevent the complication of incorporating the posterior wall in an anterior-wall suture (118).

Numerous methods and devices for the anastomosis of small vessels were developed over the following years $(138,139,79,6,7,90,97,59,74,182,125,77,78,167,195)$. Few, if any, of the mechanical "implantation-fixation" devices $(138,139,97)$ have gained wide popularity in clinical surgery. However, contributions of great value in the fields of instrumentation, suture technique and knowledge of the subject have been made by surgeons such as Buncke $(21-28,129,130$, $11,76,77,188)$, O'Brien $(146,78,147,178,159,160,148)$ and Acland $(1-10,125,172)$.

At present interrupted-suture methods are the most commonly employed in microarterial anastomoses, but there is a continuous search for improvement and simplification of anastomotic techniques. The latest experimental methods involve the use of Laser technology (195) and also the use of physiological adhesives (179).

\section{"SLEEVE" TECHNIQUES}

\section{EARLY}

As already mentioned, Murphy developed a technique for anastomosing blood vessels by inserting the proximal stump into the distal stump (135). His method is illustrated in Fig. 1.1. Among his reasons for choosing this method were the following:

1. The sutures did not have to penetrate the intima.

2. There was no suture material exposed to the blood stream. 
Murphy's experiments were called "classic" by Rich and Spencer (161) and are referred to in nearly every major historical review of vascular suraerv (118.72.81).

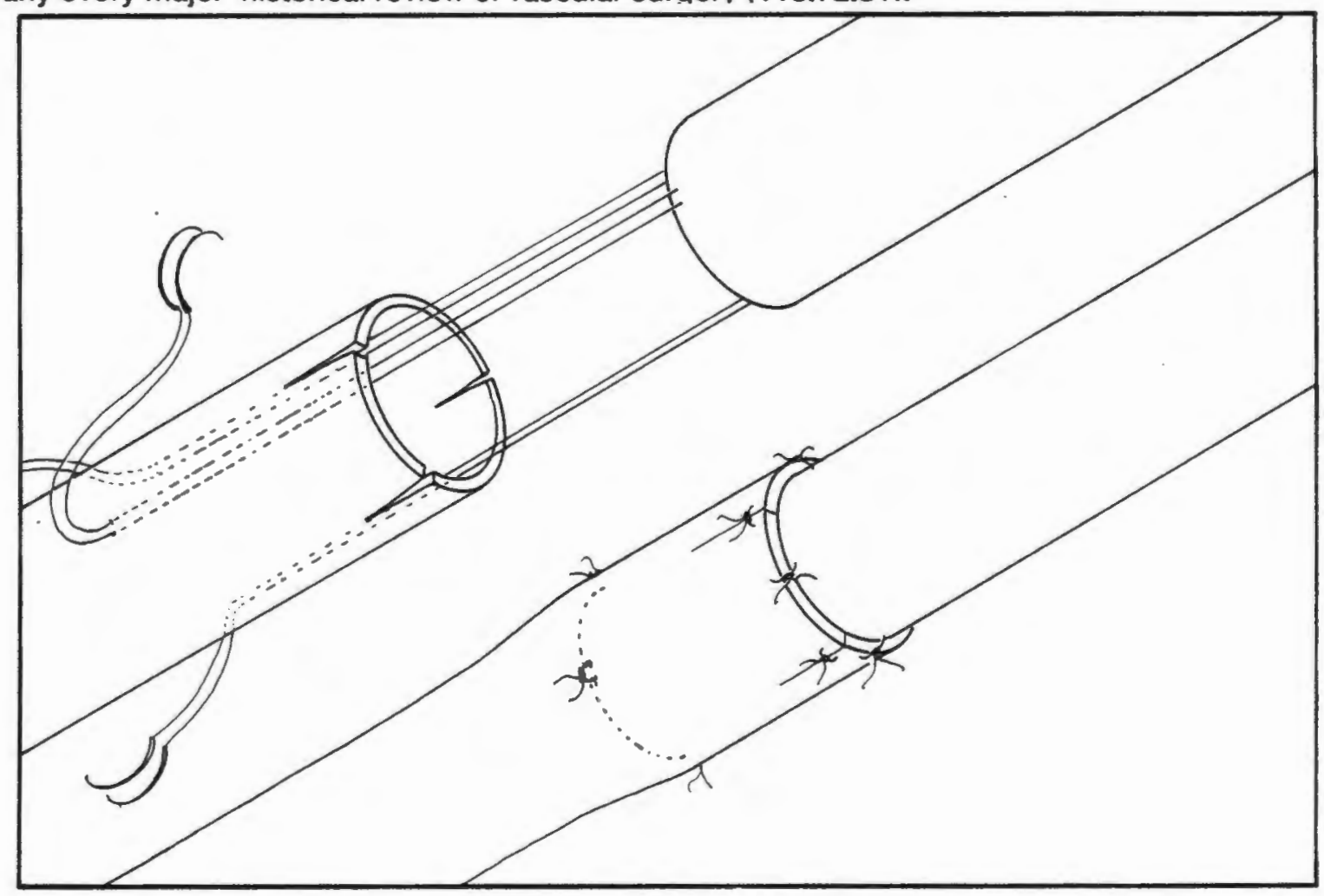

FIGURE 1.1

The anastomosis of J.B.Murphy

A few years later, in 1900, Payr introduced a method in which he passed a magnesium ring over the proximal vessel and then everted the vessel edges over the outside of the ring. This arrangement was then "plugged" into the end of the distal vessel and a ligature tied around the vessels and the magnesium ring $(154,81)$.

The method ensured intima-to-intima contact around the entire anastomosis (see Fig. 1.2). The magnesium was absorbed after some time, thus leaving the vessel unencumbered by foreign material.

Payr's method has recently been adapted for microsurgery by Naoshi Kamada and Roy Calne, who have substituted plastic tubing for the magnesium and have used the method extensively in experimental hepatic transplantation in rats (97). 


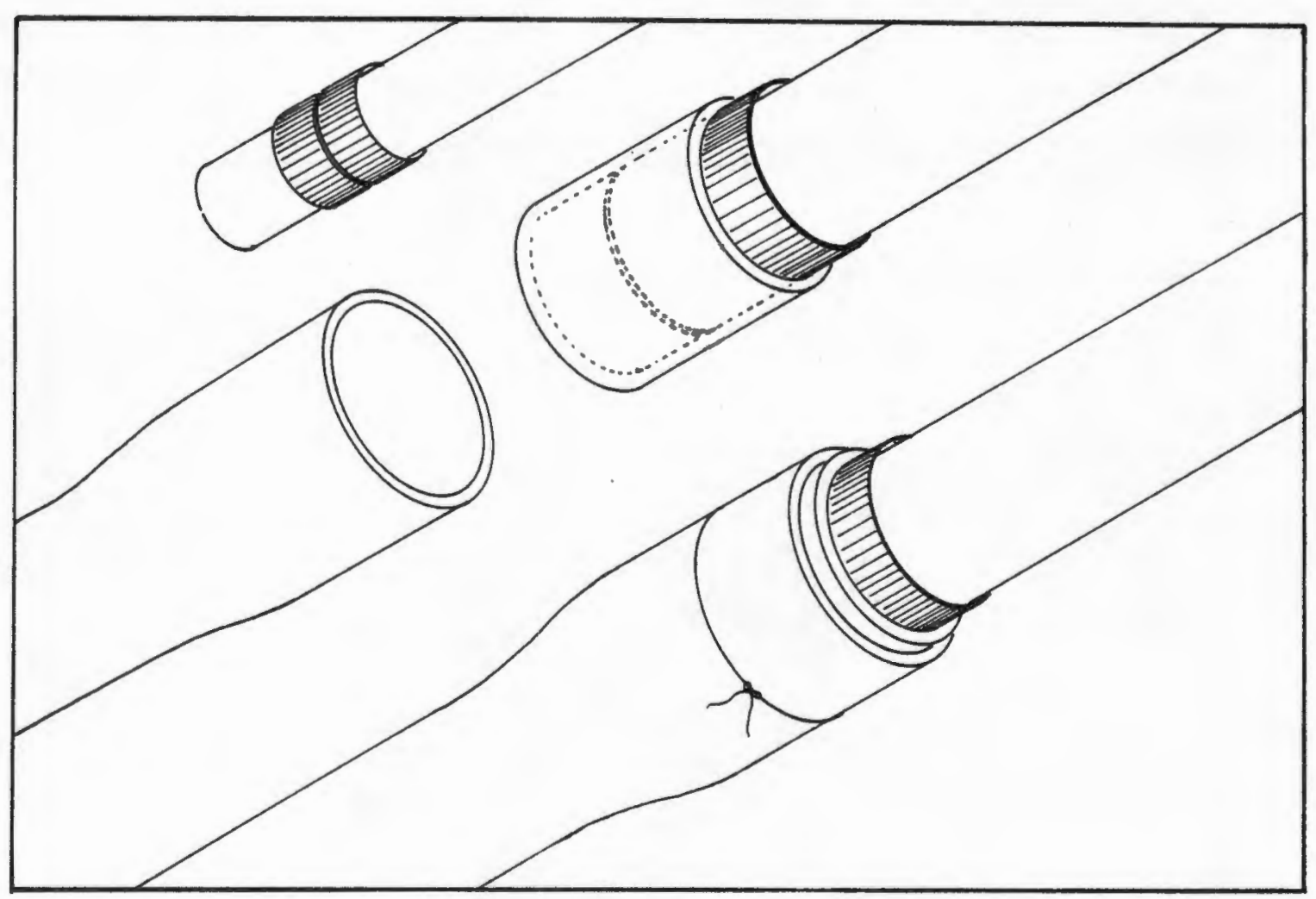

FIGURE 1.2

The method of Payr

In 1901 Bougle ${ }^{3}$ presented a telescoped anastomosis by means of which he successfully reunited transsected canine carotid arteries. Lauritzen, from whose work (107) this reference was obtained, points out that the method was very similar to the one that he had re-invented.

In 1906 Hubbard used Murphy's method to perform arteriovenous anastomoses (86) following Carrel's suggestion that compromised tissues might benefit from a reversal of the circulation through them $(33,34,35)$.

It is therefore clear that around the start of the present century methods had already been established by which one vessel could be placed within another to successfully effect an anastomosis. However, after it became apparent that vessels could be successfully anastomosed despite the exposure of suture material to the blood stream and despite damage to the intima by suture needles, these invagination methods or telescoping techniques were no longer used. 


\section{MICROVASCULAR}

In 1978 Lauritzen (112) and Meier (131) independently described microvascular anastomoses which were based on the sleeve principle. The title of Lauritzen's paper was: "A new and easier way to anastomose microvessels." and that of Meier's: "Zum anastomosenproblem kleiner Arterien." They both achieved high patency rates and Lauritzen commented on the fact that these anastomoses were achieved in a shorter time than that taken for the conventional end-toend anastomosis (ETEA). Meier, comparing blood flow through the vessels before and after 12 of his anastomoses, found that there was no change in 4 cases, an improvement in 4 cases and a mild decrease in flow in the remaining 4 cases. After histological examination of the vessels he concluded that this method of anastomosis did not, as might have been expected, cause significant stenosis of the vessel.

Lauritzen followed his first publication with four further papers in the following two years, 1979 \& $1980(108,110,109,111)$. In these he investigated his sleeve anastomosis further by histological methods and by biomechanical comparisons with standard techniques. He also described its use in clinical microsurgery. His findings indicated that the sleeve method was a viable alternative for microvascular anastomosis and that it held the advantages of timesaving and simplicity.

V.E. Meyer et al of Zurich (133) were stimulated by the work of W.E. Meier and also investigated the sleeve principle during the same period and published their findings in 1980 . They used their own technique and found that it too was a possible alternative to conventional anastomoses. At the time of publication they had already used their method in the replantation of a human thumb.

Other workers followed with their own evaluations of the sleeve principle. Some of them used methods which deviated from those already described $(41,87,176,178,204-207)$; some of these methods are shown in Fig. 1.3. Hyland (87) suggested a modification of the Lauritzen method and produced favourable results with his technique.

Stamatopoulos et al (176) used a previously undescribed technique and also produced satisfactory results. Sully et al (178) used yet another method and produced less satisfactory patency rates than their predecessors. They did not recommend their technique for clinical use. Choi et al obtained results with the sleeve anastomosis which prompted them to recommend it for use in experimental renal transplantation in rats (42). 


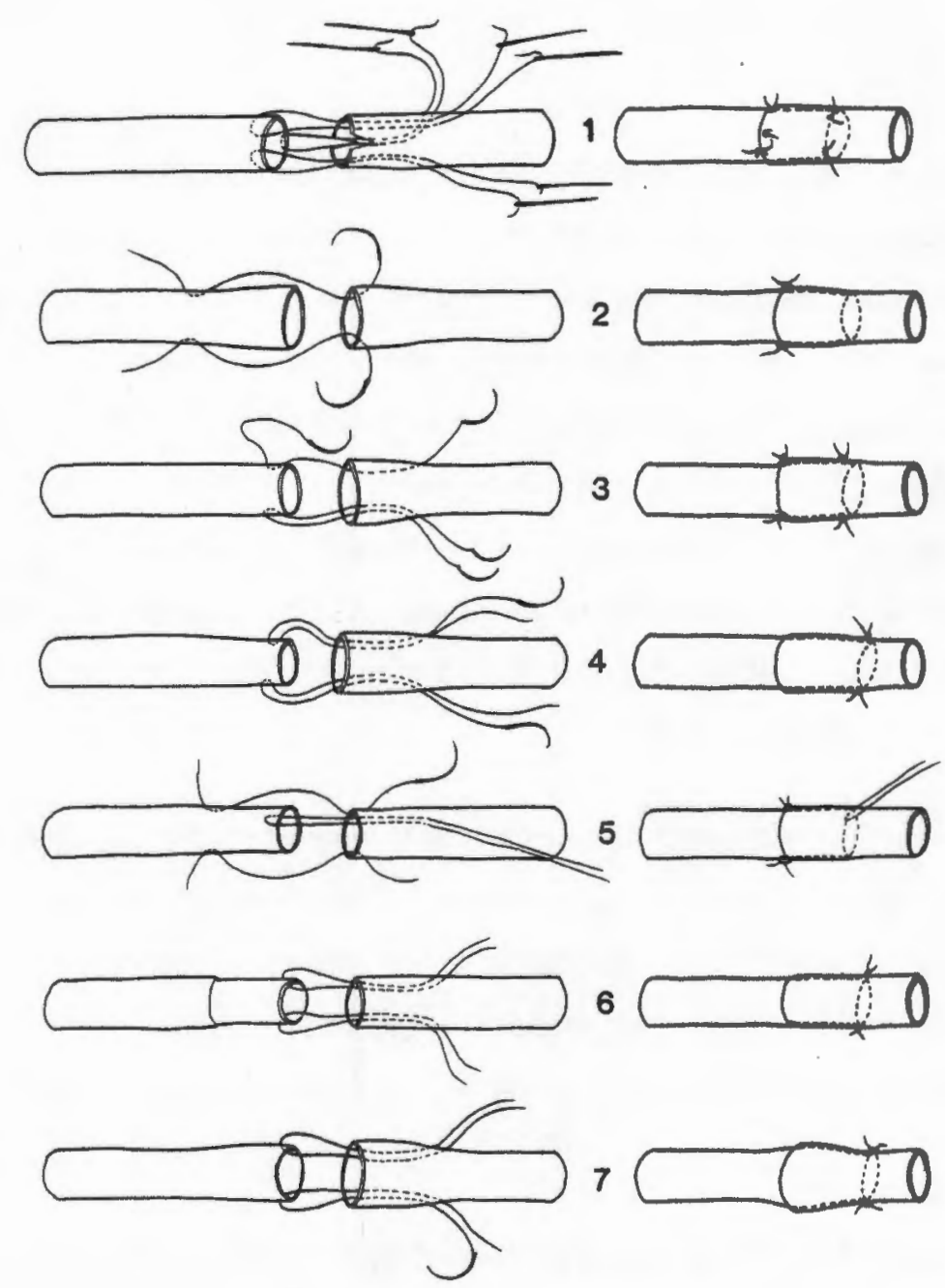

FIGURE 1.3

The variety of sleeve methods described by: 1. Murphy 2. Lauritzen 3. Meier 4. Meyer5. Hyland 6. Stamatopoulos 7. Sully

Wieslander, in association with various other workers, invested much effort in his comparative investigation of the Lauritzen sleeve anastomosis (203-207). He showed that the healing process in the sleeve anastomosis or end-in-end anastomosis (EIEA) was histologically similar to that in the end-to-end anastomosis (ETEA) (203). Patency rates were not significantly different in any of his studies, but he did find a higher incidence of partial occlusion of the vessel on the 
$7^{\text {th }}$ postoperative day in the EIEA (206) and a large reduction in flow through the EIEA in the first 3 postoperative days (204).

Another study by Wieslander et al revealed that platelet aggregation at the anastomotic site was greater in the ETEA than in the sleeve anastomosis (207).

Other workers investigated the feasibility of using the sleeve anastomosis in other structures and in other ways. Nordgren and Cohen found the method to be quick and easy for the production of arteriovenous shunts in dogs (142) and Nakayama et al have used it successfully for the same purpose in human haemodialysis patients (137). Fronticelli et al used it with success to anastomose the bile duct in hepatic transplantation in the rat (65) and Sugiura et al have used it in association with fibrin glue for the anastomoses in venous interposition grafts (179).

The application of the sleeve anastomosis in clinical microvascular surgery has been reported in limited numbers of cases by Lauritzen et al (110), Meyer et al (133), Vilkki (197) and in a large number of cases by Nakayama et al (137) and Prof. Zhong-Wei Chen. ${ }^{4}$

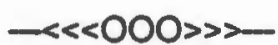

NOTES

1. C.Lauritzen, personal communication.

2. Smith S.: Studies in experimental vascular surgery. Surgery 18:627-, 1945. (from 118).

3. Bougle J. La suture arterielle. Arch Md Exp Anat Path XIII, 205. (Ref. from Lauritzen, 107). 
4. At the Fifth congress of the European Section of the International Confederation for Plastic and Reconstructive Surgery, held in Stockholm, $16^{\text {th }}$ to $20^{\text {th }}$ June 1985. 


\title{
CHAPTER II \\ QUESTIONS \& OVERVIEW OF APPROACH
}

\author{
"Clever people seem not to feel the natural pleasure of \\ bewilderment, and are always answering questions when \\ the chief relish of life is to go on asking them."
}

Frank Moore Colby(1926)

\section{PREVIOUS EIEA TECHNIQUES}

Since 1978 many of the aspects and attributes of the EIEA have been studied and evaluated. It is, however, to be noted that, as shown in Fig. 1.3, most of the workers who originally set out to evaluate the concept of EIEA used suture placement methods which differed from that which had been described by Lauritzen (112).

Small vascular structures are highly intolerant of poor Surgical technique and afford the surgeon very little room for recovery or "cover-up" manoeuvres where mistakes or errors of judgment have been committed. Consequently microvascular surgery is taught as a highly disciplined system of procedures, in which attention is given to every detail of every movement of the surgeon.

There are at present only a few correct ways in which to perform any one of the group of manoeuvres constituting microsurgical technique. However, a number of different methods for "skinning the EIEA cat" have emerged and this plethora of methods poses the following two major questions:

1. Did other workers encounter difficulties in using Lauritzen's original technique and therefore try to develop alternatives? 


\section{Chapter 2 - Question and Overview of Approach}

2. Was any one of the new methods an improvement on the Lauritzen technique?

\section{QUESTION 1}

Did other workers encounter difficulties in using Lauritzen's original technique and therefore try to develop alternatives?

The following quotations may give an indication of the problems occasionally experienced by Lauritzen in the course of using his own technique.

In the Abstract of the primary article (112) he wrote:

"A new technique for microvascular anastomosis, telescoping one vessel into the other requiring only 2 sutures is described."(MI)

In a letter to the Editor (114), replying to adverse criticism of the "sleeve" anastomosis by Sully et al (178), he stated :

"Further, they introduced intraluminal sutures and then added extra sutures to make sure that the blood stream had no chance to adjust the two vessels into proper position."(MI)

He went on to say :

"The sleeve anastomosis uses two or three sutures, all of them extraluminal." (MI)

In a "discussion" of a paper by Wieslander and Rausing (206), Lauritzen said (113) :

"As in this paper, having to add extra sutures to prevent leaking from an anastomosis between two equally sized vesseis usually is an indication of an improperly made anastomosis. The authors describe that they put two sutures in at 120 degrees apart and then allowed the blood stream to flow. This is a technical error, and it must be rather unusual in such a situation that the anastomosis does not leak or that the sleeve proximal stump does not jump out." (MI)

In a paper (109):

"In this series 3 stitches instead of two were used ..."

In another paper (108): 
"On the other side the sleeve anastomosis was made using 3 evenly spaced stitches."

...and later in the same paper:

"Within the first postoperative hours, however, the proximal end of one of the sleeve anastomoses slid out and three of the end to end anastomoses began to leak." (MI)

In a case report (110):

"Two sutures were used at first, but when changing the flap position the recipient artery jumped out. After reinsertion 2 additional sutures were placed giving sufficient stability."

The foregoing quotations imply that Lauritzen himself occasionally experienced problems in effecting a "stable" anastomosis. The same is likely to occur in the hands of other workers.

It is also clear that, as shown in the clinical case study, the stability or lack thereof is not always predictable. "Slipping-out" of the feeding-vessel (FV) stump, or the "jump-out" phenomenon, can be a most disastrous complication in microsurgery for the following reasons:

(A) The pulsating jet of blood trequently strikes the microscope lens cover rendering the operating field partially invisible for as long as it takes to clean up.

(B) The open ends of the vessels lie in a pool of clotting blood, from which they have to be retrieved, sometimes blindly. Such a situation is fraught with the following dangers :

1. thrombus formation within the vessel;

2. mechanical damage to the vessel during retrieval attempts;

3. clamps have to be re-applied, which may cause intimal damage; and

4. the vessels have to be re-manipulated into their telescoped position, again with the possibility of mechanical damage.

Lauritzen points out that the two sutures in his anastomosis should be placed exactly 180 degrees apart (112). Theoretically this would cause the intraluminal pressures on either side of a plane passing through the two sutures to be exactly equal, thus forcing the FV open inside the lumen of the receiving-vessel (RV) and denying it the opportunity of slipping out on either side 
of the suture plane. However, the required degree of accuracy is difficult to attain and to maintain.

The possibility and the unpredictability of a "jump-out" must be even greater in the hands of surgeons who are less experienced in the use of this form of anastomosis. It is therefore not surprising that many who have been "once bitten" elect to try a slightly different method.

A second less obvious problem is that of intimal damage occurring in the inner vessel during the manipulations required to insert the FV into the receiving stump. Lauritzen advocates the use of one or both limbs of a fine forceps to push the FV into the receiving stump (112) and the technique obviously works well for him, but in the hands of surgeons with only slightly less dexterity, this manoeuvre, taking place within the confines of a $1 \mathrm{~mm}$ blood vessel, can be extremely hazardous to the delicate intimal layer of the FV.

Once again a hint of the problem's existence may be found in the published work of Lauritzen (108):

\footnotetext{
"The single thrombosis found in the sleeve anastomosis series $(n=42)$ may be explained by additional manipulations needed to establish flow immediately postoperatively. The operating time in this case was 45 $\min . "(M B)$
}

These two complications which may arise with employment of the Lauritzen technique detract from its most elegant simplicity and tend to negate its main advantage of timesaving.

It may, therefore, be concluded that, if Lauritzen experienced occasional problems with his own technique, those surgeons who were less familiar with it, would have done so too.

\section{QUESTION 2}

Was any one of the new techniques an improvement on the original technique in the hands of the average microsurgeon?

The question of defining "improvement" is in itself wide-ranging. A modification which distinctly facilitates one part of the whole procedure may constitute a retrogression in another component. In such cases the summation of apparent gain and apparent loss may yield an improvement which is only apparent. 
A substantive improvement would be one in which nothing is lost while even the smallest gains are made.

The technique of Lauritzen makes full use of all the ascribed advantages of the EIEA and it is therefore extremely difficult to improve upon its adherence to those principles. It is noteworthy that not one of the modifications or different methods pretends to improve upon the utilisation of advantages. Of further interest are the methods which glibly abandon some of the advantages in pursuit of fewer mechanical complications. An example of such a method is the one described by Hyland et al (87), in which the problems of the manual invagination of the vessel ends and the "jump-out" are both addressed by means of a third suture, which acts as a guiding suture. This guiding suture may be considered to constitute:

1. An extra suture which takes extra time.

2. An assault upon the intima of the FV, as the suture is depicted as passing through the entire wall-thickness of the FV.

3. Additional intimal trauma areas, which are exposed to the blood stream at those areas where the suture twice passes through the wall of the receiving vessel. As the inner blood vessel retracts after the removal of the guiding suture, those holes and areas of intimal damage will be exposed.

The other four techniques $(131,133,176,178)$ have as a common factor looped sutures, which hold the end of the FV deep inside the stump of the RV (Fig. 2.1). There are always at least two loops placed 180 degrees from each other, each -

1. passing through the wall of the RV 1 to 2 vessel diameters from the cut end;

2. taking a bite of the end of the FV near its cut end; and

3. coming back through the lumen of the RV and passing through its wall adjacent to the first pass.

Two of these methods have the bite of the end of the FV passing tangentially through the media $(133,176)$, while the other two fail to avail themselves of the opportunity of avoiding the presence of foreign material in the active vascular lumen by passing their loops through the full thickness of the FV's wall $(131,178)$ 
In one of each of these loop-method pairs additional sutures have been employed to hold the end of the RV to the side of the FV $(131,178)$. This practice increases the operating time and may prevent adjustment between the vessel walls once they are subjected to the stresses of pulsatile flow in their lumina (114).

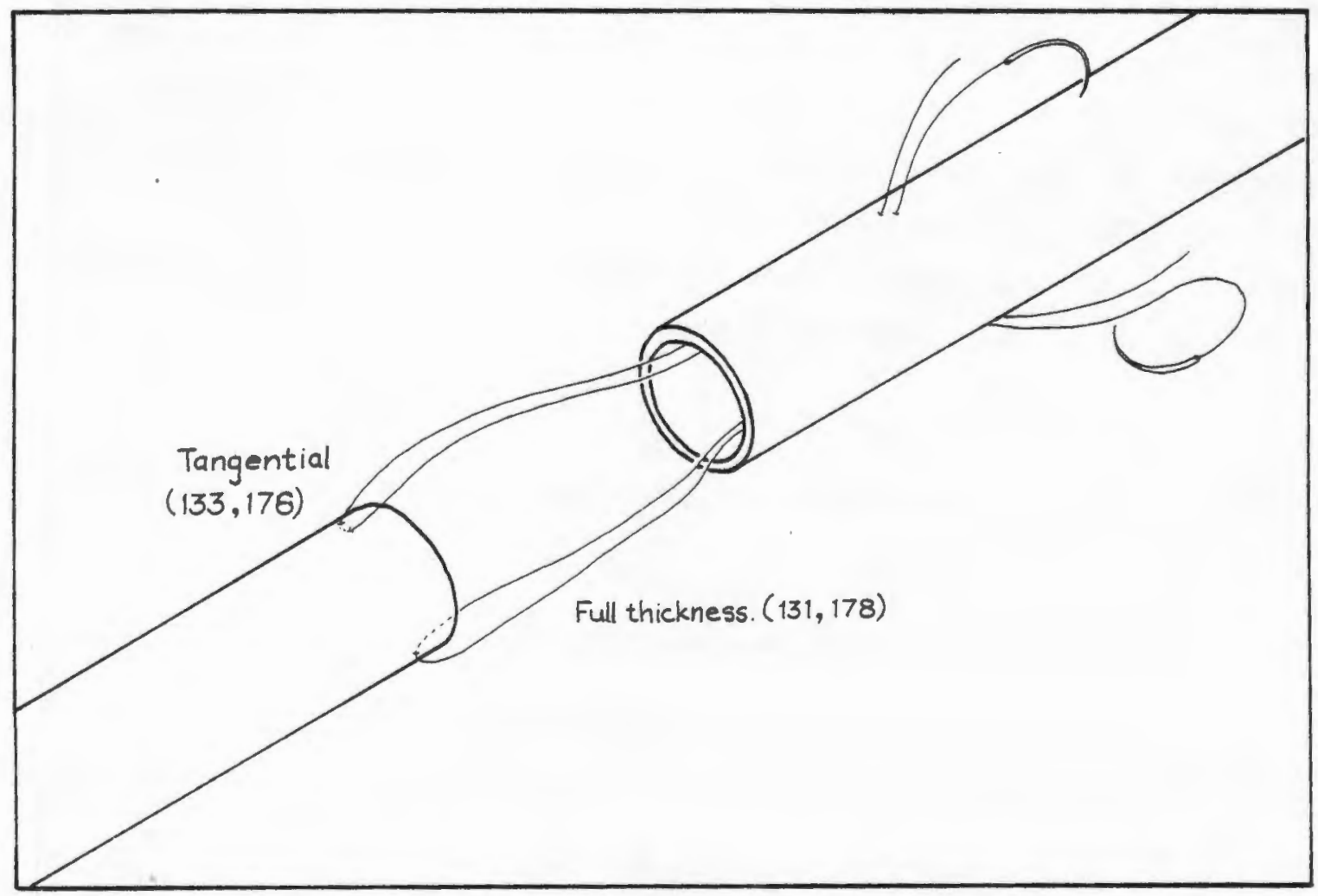

FIGURE 2.1

Looped suture detail

In all the methods using two looped sutures deep in the lumen of the RV, there is a real danger of getting elements of the loops entwined within the lumen while placing the sutures. Such an occurrence gives rise to difficulty in sliding the FV into the RV stump and may, when the sutures are firmly tied, cause complete occlusion of the RV (Fig. 2.2). The only way to correct an intraluminal entwinement is to remove at least one loop completely and then to replace it with a new one, thus losing time and increasing mechanical damage in the process.

When extra sutures have not been used to fix the end of the RV to the side of the FV and the loops have not been placed exactly 180 degrees apart on both stumps, the RV sometimes slips back to expose a slightly lax edge of the FV and this results in a torrential leak. 
There is, therefore, only one of these four methods which embraces the advantages of the EIEA as effectively as Lauritzen's method and that is the method of Meyer et al. Like Lauritzen they stress the importance of placing the sutures exactly 180 degrees apart and indicate that there are complications if the desired accuracy is not achieved. Unfortunately their method of suture placement is more complicated and has the inherent danger of intraluminal entwinement.

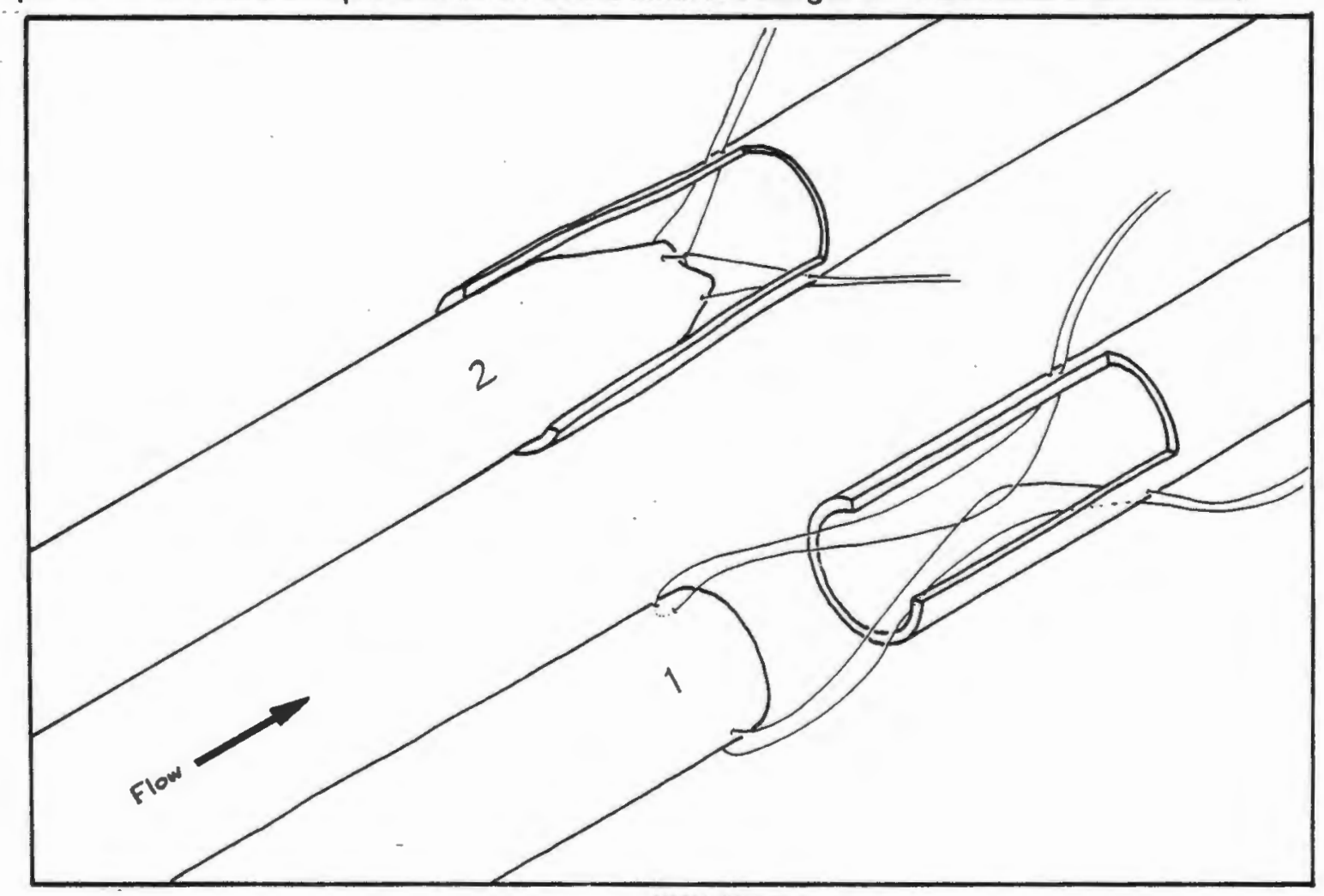

FIGURE 2.2

Entwined loops causing occlusion

It may, therefore, be concluded that not one of the methods, new or modified, represents a real improvement on the technique of Lauritzen, although the technique of Hyland et al (87) is a laudable attempt. 


\section{THE BASIC QUESTION}

\section{ADVANTAGES OF THE EIEA}

The inherent advantages attributable to the EIEA concept are the following:

1. The absence of foreign material (suture) exposed to the blood stream.

2. The absence of intimal trauma by needles or suture material in the areas of contact between blood and intima.

3. A significant decrease in the total number of sutures used, leading to:

a) less scarring of the vessel wall;

b) less trauma of manipulation;

c) timesaving;

d) suture material saving; and

e) less impairment of vessel growth.

4. Prevention of intimal separation.

5. Prevention of aneurysm formation at the point of anastomosis.

The answers to our first two questions have shown that the methods proposed by Lauritzen and by Meyer et al both embrace these principles, but that each of them has, especialiy in less experienced hands, potential for a serious mechanical complication, which could negate the positive aspects of the concept and even ruin the repair completely.

If a new concept can simplify a procedure, the most benefit from its use will be gained by those who are experiencing difficulty with the execution of the procedure in its standard form.

"A new and easier way to anastomose microvessels" (112) should therefore be of primary benefit to the population of novice microsurgeons and those who have, for lack of regular 
practice or for physical reasons, less than the keenest microsurgical skills. It is a pity that in their hands the EIEA will probably be fraught with greater difficulties than will the standard ETEA techniques.

This brings us to the question around which this thesis is based:

Is it possible to design and develop a suture-placement technique which will: effect an EIEA,

embrace all the conceptual advantages,

eliminate the existing mechanical complications

and

be clinically as effective as a standard ETEA

so that it will truly be:

A New and Easier Way to Anastomose Microvessels?

\section{APPROACH}

"If a way to the Better there be, it exacts a full look at the

Worst."

Thomas Hardy(1901)

As suggested by Hardy (albeit in a slightly different context), the existing EIEA methods of Lauritzen, Meyer and Hyland were carefully re-examined. By repeatedly practising each technique in the rat femoral artery, with meticulous attention to every detail provided in the original descriptions, it was possible to become reasonably proficient in the execution of each of 


\section{Chapter 2 - Question and Overview of Approach}

the techniques. During this period of practice the mechanical complications described in the previous section occurred a few times in each group.

The "mistakes" or technical difficulties leading to the development of each of the complications were identified and noted wherever possible. In several cases, especially with the Lauritzen technique, a seemingly excellent anastomosis fell prey to the dreaded "jump-out". Similar catastrophes occurred on two occasions after the guiding suture of the Hyland technique had been removed for more than 20 minutes. Problems with the Meyer technique were usually discovered before release of the clamps, but here also there were two episodes of totally unpredicted catastrophic bleeding.

During the exercise it became possible to make definite but subtle prospective mistakes which would predictably lead to one of the complications.

Armed with the knowledge of both the spontaneous and contrived errors and of the pitfalls, it became possible to embark upon the design of a new technique.

When this technique had been designed, it had to be tested to determine whether:

1. there were any hidden pitfalls;

2. the anastomosis compared favourably with the standard techniques in terms of patency, overall function and operating time;

3. there were fewer complications experienced by less accomplished microsurgeons, when compared to the previous EIEA methods; and

4. there were other yet unexplored applications to which the new anastomosis might be suited because of its unique characteristics.

The bulk of this thesis will be devoted to the development of the new anastomotic technique and descriptions of the various experimental systems designed to test the anastomosis, together with the results obtained.

A new microsurgical instrument of great simplicity and even greater value was designed to assist in the anastomotic technique. This instrument has subsequently been found to be well suited to a number of other tasks. To evaluate and compare the results of different types of experimental anastomoses, a simple new method involving corrosion casts and photographic 
experimental anastomoses, a simple new method involving corrosion casts and photographic techniques was developed. These methods and materials will each be described in an appendix.

$-==<<000>>==-$ 
Chapter 2 - Question and Overview of Approach 


\section{CHAPTER III}

\section{THE NEW ANASTOMOSIS}

"It is common sense to take a method and try it: if it fails, admit it frankly and try another."

- Franklin D. Roosevelt

As mentioned in the previous chapter, the first step in the development of the new technique was a careful analysis of the pitfalls encountered in the existing procedures. Only those techniques which utilised the full potential of the EIEA concept were studied $(112,133)$. An exception was made for the Hyland technique (87), which was modified slightly to minimise its loss of advantages and then included in the study, as it was the only method which had been designed to address the same problems I intended to tackle.

Having studied the existing techniques and having noted both their advantages and shortcomings, I could then proceed to attempt the design of a technique which would satisfy all the criteria for a good EIEA.

\section{EXAMINATION OF EXISTING TECHNIQUES}

I had, before the commencement of this study, performed the EIEA numerous times by means of each of the three methods. The procedures were carried out on the femoral arteries of adult male Sprague-Dawley rats. The external diameters of the vessels ranged from $0.8 \mathrm{~mm}$ to $1.2 \mathrm{~mm}$ on rough estimation, using obliquely cut $1 \mathrm{~mm}$ squared EKG paper ${ }^{1}$ as a guide (Fig. 3.1 )

No attempt was made to measure operating time, the accent in all cases being placed on accuracy of every step of the procedure. There was also no attempt made to study the anastomoses over a longer period; in fact, the animals were killed $40 \mathrm{~min}$. after the completion of the anastomoses while they were still deeply anaesthetised. A note on each step was 
dictated throughout the procedure to eliminate the role of the "retrospectoscope." The results immediately after clamp release and within the following 30 minutes were also recorded.
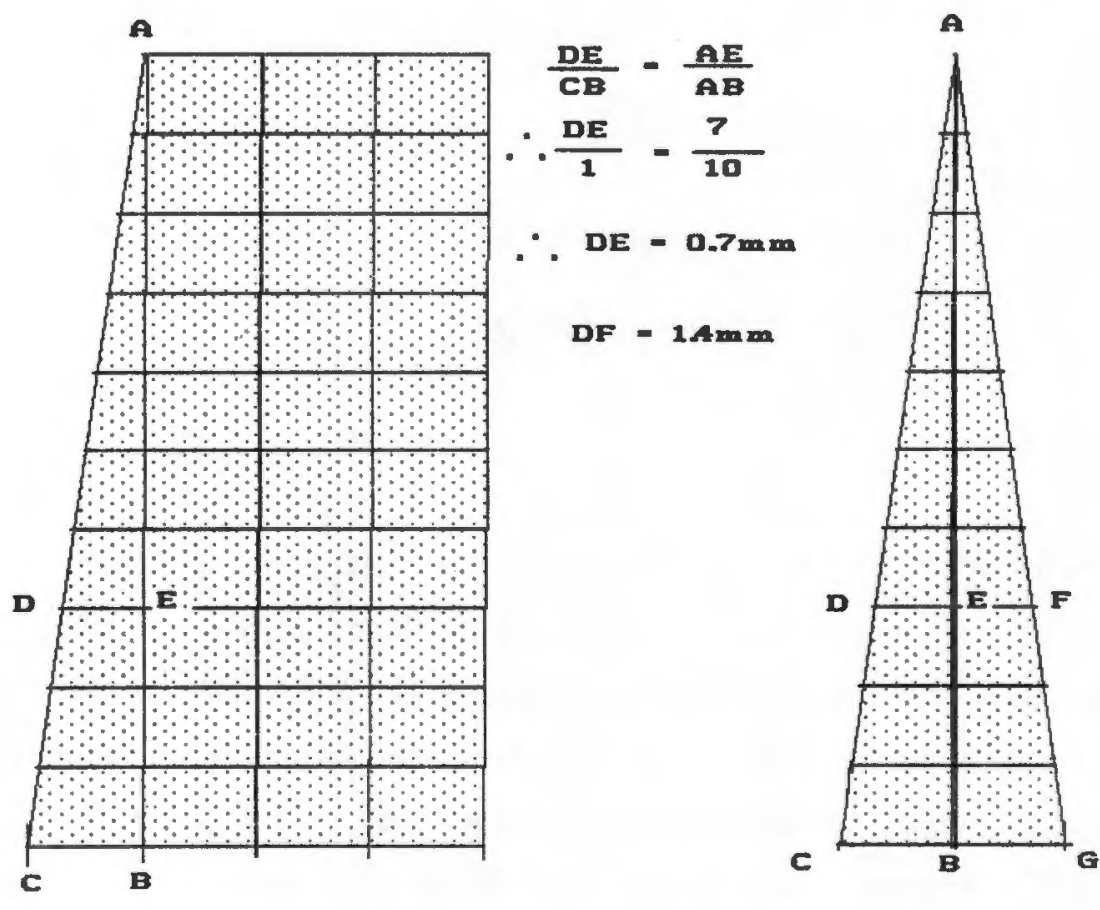

FIGURE 3.1

Obliquely cut EKG paper measurement guide.

\section{THE LAURITZEN TECHNIQUE}

This technique (Fig. 3.2) consists of the following steps compiled from different publications by Lauritzen $(112,115)$ :

* place the vessel ends in an approximating bar- clamp ;

* $\quad$ clear the adventitia from the vessel ends;

* dilate both stumps with Acland's vessel dilators; 

vessel, 1 to 2 vessel diameters from the cut end, passing towards the end through adventitia and media only, not breaching the intima, then going on to pass through the wall of the receiving vessel in the same way as the second half of a normal end-to-end anastomosis suture (i.e. from inside to outside);

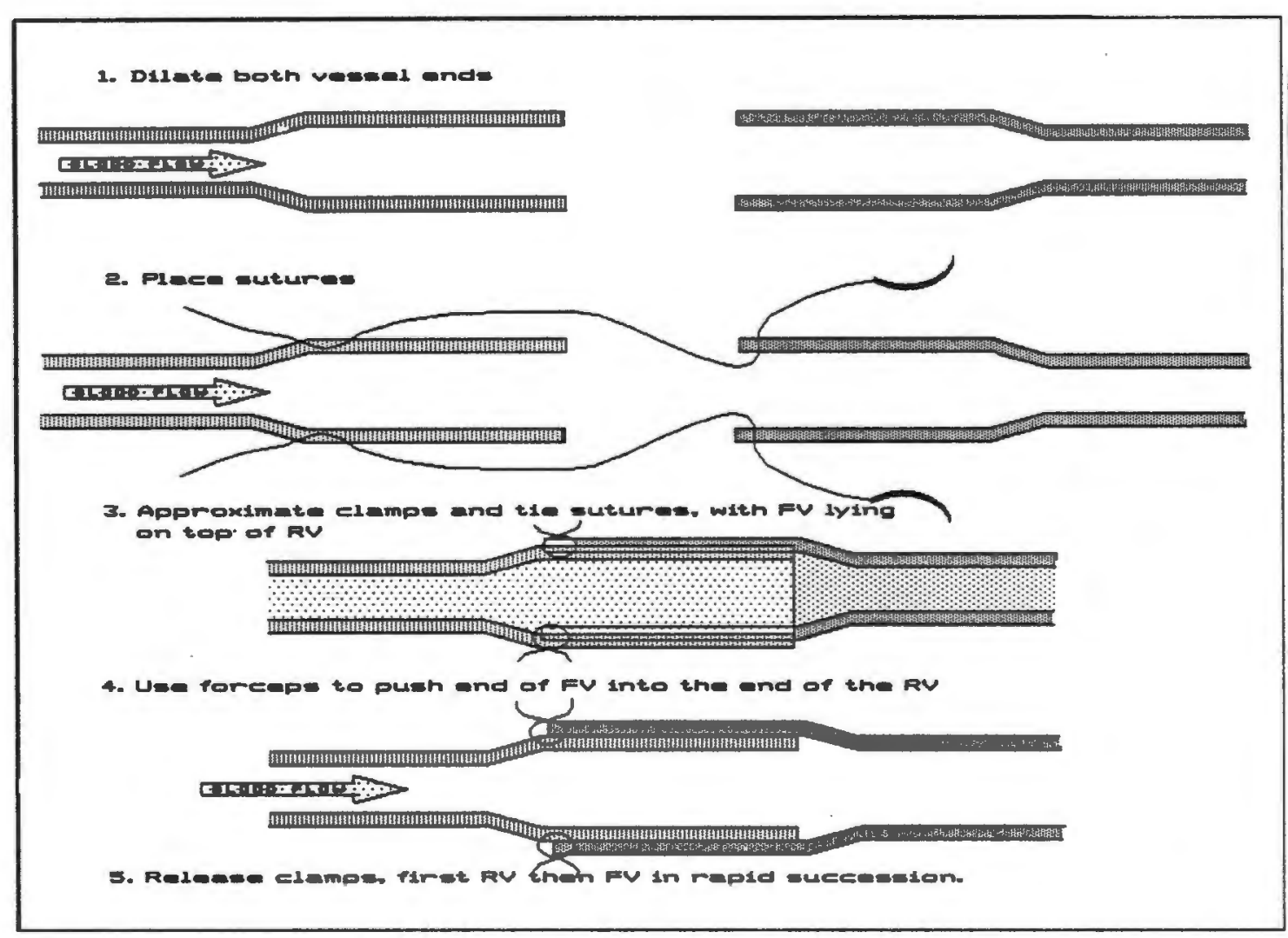

FIGURE 3.2

Lauritzen technique, all steps.

* place a similar suture exactly 180 degrees from the first suture;

* approximate the clamps on the bar so that the vessel ends overlap each other by 1 to 2 vessel diameters;

* $\quad$ place the feeding vessel stump on top of the receiving vessel stump (see Fig. 3.2 Step 3); 
* tie both sutures;

* using two pairs of fine jeweler's forceps, one for handling each stump, pick up the feeding vessel end and tuck it into the receiving vessel;

* ensure that all wrinkles and folds in the feeding vessel are straightened out by probing with a limb of the fine forceps; and

* release the clamps, first the receiving vessel clamp, followed immediately by that of the feeding vessel.

By using this method 32 anastomoses were made. The first 20 were made as close to perfect as my capabilities would allow. The last 12 had small deliberate imperfections introduced so that their consequences might be witnessed.

The suture-separation angle was calculated roughly by measuring the distance between the completed sutures and then comparing this distance with the measurement across the collapsed vessel (see Fig. 3.3).

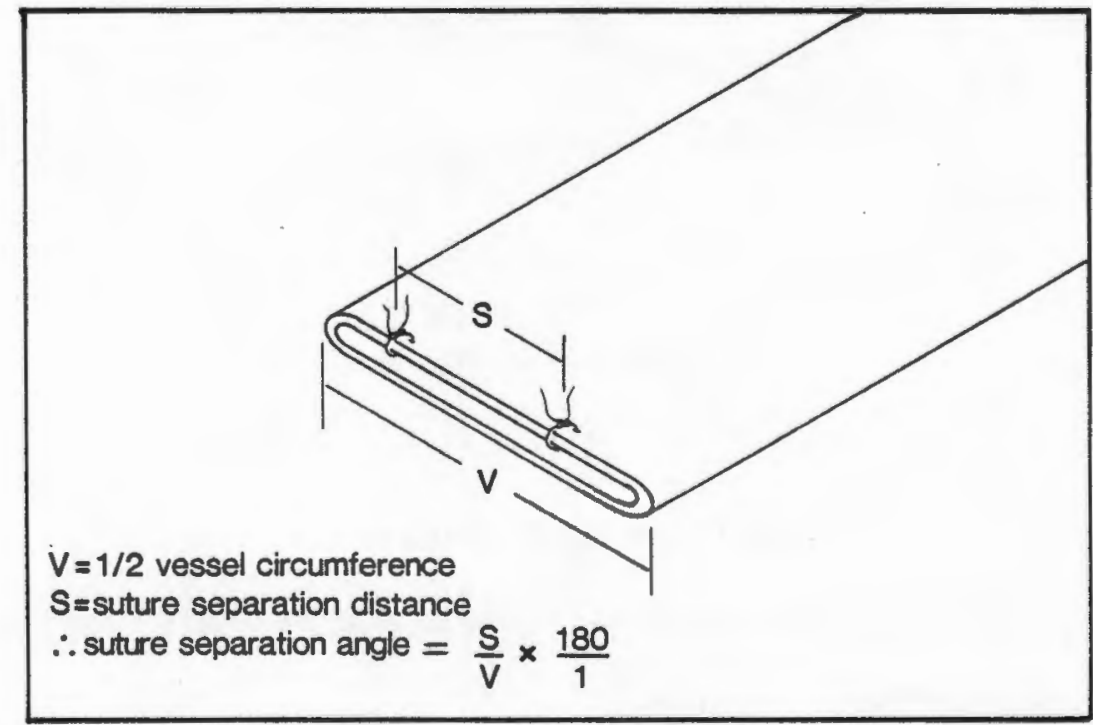

FIGURE 3.3

Measuring suture-separation angle. 
Intra-operative notes and results are shown in Fig. 3.4

The following points were noted in the first twenty anastomoses, all of which were carried out with the greatest possible care :

Four (20\%) developed complications in the first 30 minutes.

In two of those four cases, \#4 \& \#14, there was no reason to suspect that anything might go awry.

There were five cases (25\%) in which some difficulty was experienced with insertion of the feeding vessel into the receiving vessel.

Three of those five, \#2, \#7 \& \#19, had overlaps longer than 1.5 vessel diameters.

In one of the five cases, \#11, there was no accountable reason for the difficulty. During the process of insertion the superficial bite of the suture in the feeding vessel was torn out and the suture had to be replaced. Thereafter the insertion was reattempted and was eventually successful despite difficulties. Within 30 minutes post clamp release the vessel was totally occluded, probably due to excessive manipulation with resultant intimal trauma.

The jump-out which occurred in \#20 might be attributable to the fact that the sutures were not 180 degrees apart, but in vessels of the magnitude of $1 \mathrm{~mm}$ diameter it should be taken into consideration that 10 degrees is spanned by a distance of less than one tenth of a millimetre. (In comparison it should be noted that the sutures of a standard end-toend anastomosis are placed 45 degrees or more apart in such a vessel). The $10^{\circ}$ error in suture placement was therefore not gross.

In cases \#15 to \#20 the adventitia of the receiving vessel was not trimmed. Cases \#15 to \#19 indicated clearly that trimming of the RV adventitia was not important for the success of the anastomosis. (The \#20 jump-out could logically not be attributed to the lack of adventitial trimming). 
Chapter 3 - The new EIEA: Development \& Design

\begin{tabular}{|c|c|c|c|c|c|c|c|c|c|c|c|c|c|c|}
\hline CASB & \begin{tabular}{|l} 
ADVERII \\
TRIKIIII \\
PV
\end{tabular} & & \begin{tabular}{|l} 
YBSSE \\
DILA \\
PV
\end{tabular} & & $\begin{array}{l}\text { PIRST } \\
\text { SUIVI } \\
\text { PV }\end{array}$ & & $\begin{array}{l}\text { SECO } \\
\text { SUYU } \\
\text { PY }\end{array}$ & $\mathbf{R V}$ & $\begin{array}{l}\text { DBGREES } \\
\text { APART }\end{array}$ & $\begin{array}{l}\text { OYBR - } \\
\text { LAP }\end{array}$ & $\begin{array}{l}\text { YUCR } \\
\text { III }\end{array}$ & $\begin{array}{l}\text { KBCH. } \\
\text { DAYAGB }\end{array}$ & $\begin{array}{l}\text { RBSULT } \\
\text { IMBDUTH }\end{array}$ & $\begin{array}{l}\text { RBSULT } \\
30 \text { KIII }\end{array}$ \\
\hline $\begin{array}{l}1 \\
2 \\
3 \\
4 \\
5 \\
8 \\
9 \\
8 \\
9 \\
10 \\
11 \\
12 \\
13 \\
14 \\
15 \\
16 \\
19 \\
18 \\
19 \\
20\end{array}$ & $\begin{array}{l}y \\
y \\
y \\
y \\
y \\
y \\
y \\
y \\
y \\
y \\
y \\
y \\
y \\
y \\
y\end{array}$ & $\begin{array}{l}! \\
! \\
! \\
! \\
! \\
! \\
! \\
! \\
! \\
! \\
1 \\
1 \\
1\end{array}$ & $\begin{array}{l}y \\
y \\
y \\
y \\
y \\
y \\
y \\
y \\
y \\
y \\
y \\
y \\
y \\
y \\
y \\
y \\
y\end{array}$ & $\begin{array}{l}y \\
y \\
y \\
y \\
y \\
y \\
y \\
y \\
! \\
! \\
! \\
! \\
! \\
y\end{array}$ & 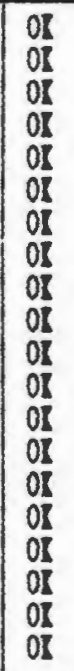 & $\begin{array}{l}01 \\
01 \\
01 \\
01 \\
01 \\
08 \\
01 \\
01 \\
01 \\
01 \\
01 \\
01 \\
08 \\
08 \\
08 \\
08 \\
01 \\
01 \\
01 \\
01\end{array}$ & 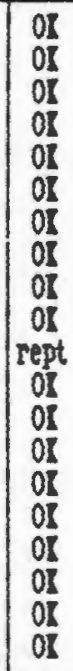 & 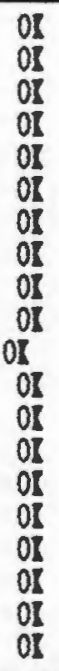 & $\begin{array}{l}175-185 \\
170-180 \\
175-185 \\
175-185 \\
175-185 \\
170-180 \\
175-185 \\
175-185 \\
170-180 \\
175-185 \\
175-185 \\
175-185 \\
175-185 \\
190-180 \\
170-180 \\
195-185 \\
175-185 \\
175-185 \\
175-185 \\
165-175\end{array}$ & $\begin{array}{l}1.5 \\
2.0 \\
1.2 \\
1.5 \\
1.5 \\
1.5 \\
2.0 \\
1.3 \\
1.5 \\
1.7 \\
1.5 \\
1.2 \\
1.7 \\
1.3 \\
1.5 \\
1.5 \\
1.3 \\
1.5 \\
1.8 \\
1.5\end{array}$ & 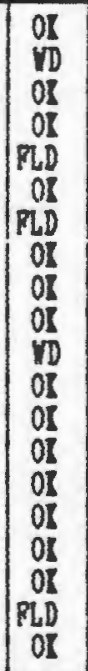 & 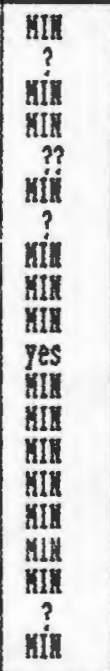 & 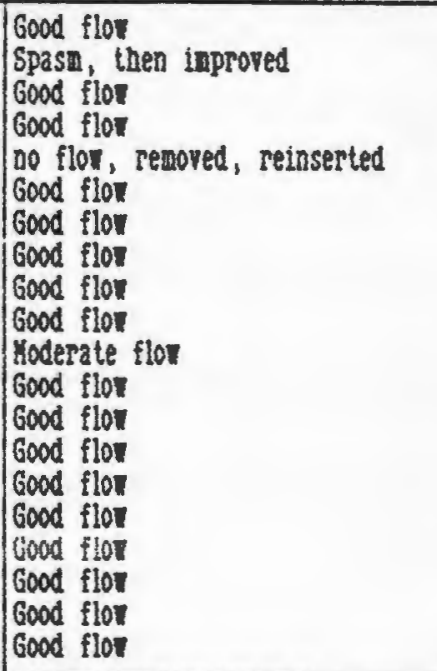 & 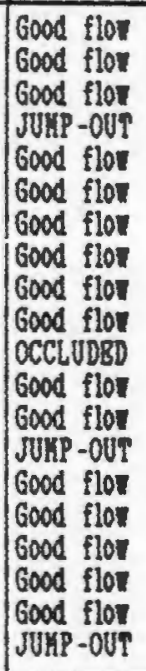 \\
\hline $\begin{array}{l}21 \\
22 \\
23 \\
24 \\
25 \\
26 \\
29 \\
28 \\
29 \\
30 \\
31 \\
32\end{array}$ & $\begin{array}{l}y \\
! \\
1 \\
1 \\
1 \\
y \\
! \\
! \\
y\end{array}$ & $\begin{array}{l}y \\
y \\
y \\
y \\
1 \\
1 \\
1 \\
1 \\
1 \\
1 \\
1\end{array}$ & $\begin{array}{l}1 \\
y \\
y \\
y \\
y \\
y \\
y \\
y \\
y\end{array}$ & $\begin{array}{l}y \\
y \\
y \\
y \\
y \\
y\end{array}$ & $\begin{array}{l}01 \\
01 \\
01 \\
01 \\
01 \\
01 \\
01 \\
01 \\
01 \\
01 \\
01 \\
01 \\
01 \\
01\end{array}$ & 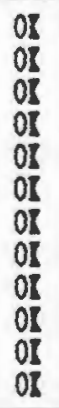 & $\begin{array}{l}01 \\
01 \\
01 \\
01 \\
01 \\
01 \\
01 \\
01 \\
01 \\
01 \\
01 \\
01 \\
\text { or }\end{array}$ & $\begin{array}{l}\text { or } \\
\text { of } \\
\text { of } \\
\text { oI } \\
\text { oI } \\
\text { of } \\
\text { of } \\
\text { of } \\
\text { of } \\
\text { of } \\
\text { of }\end{array}$ & $\mid \begin{array}{c}175-185 \\
175-185 \\
175-185 \\
175-185 \\
175-185 \\
165-175 \\
160-170 \\
155-165 \\
140-150 \\
160-1 \\
175-185 \\
180\end{array}$ & $\begin{array}{l}1.5 \\
1.5 \\
1.3 \\
1.5 \\
1.5 \\
1.5 \\
1.5 \\
1.5 \\
1.7 \\
1.5 \\
1.0 \\
0.7\end{array}$ & 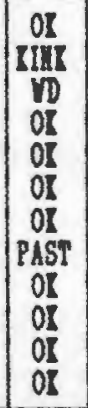 & 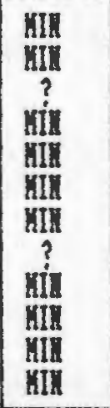 & 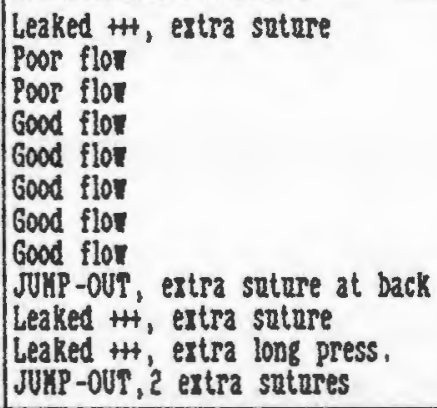 & 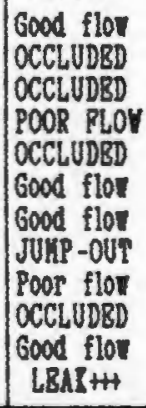 \\
\hline
\end{tabular}

FIGURE 3.4

Lauritzen technique, results.

In cases \#21 to \#32 specific mistakes or omissions were made to observe their effects. The following facts were noted:

Failure to dilate the feeding vessel resulted in excessive leaking, which in this case (\#21) required an extra suture to achieve control. Lauritzen has pointed out that both vessels should be dilated $(112,115,113,116)$.

If the inner vessel is not dilated, it will be less compliant than the outer vessel, thus leading to a loss of the desired close apposition between the vessel walls and this predisposes to leaking. An undilated vessel also has a greater wall-thickness to lumen ratio, which could mean that relatively more of the cut edge, un-covered by intima, is exposed to the blood stream. The exposed sub-intimal tissues would be thrombogenic. 
Failing to dilate either of the two vessels could give rise to complicated insertion as well as excessive stenosis at the anastomotic site. It is definitely of vital importance to dilate the receiving vessel (\#22 \& \#23).

If the adventitia of the feeding vessel was not trimmed, \#24 \& \#25, the anastomosis appeared normal after clamp release, but in both cases there was a marked deterioration within the next 30 minutes. This poor performance can almost certainly be ascribed to the thrombogenic effect of the damaged adventitial fronds wafting in the blood stream.

In cases \#26 \& \#27 an attempt was made to place the sutures less than 180 degrees apart by a small margin. Surprisingly, cf. \#14 \& \#20, there were no complications!

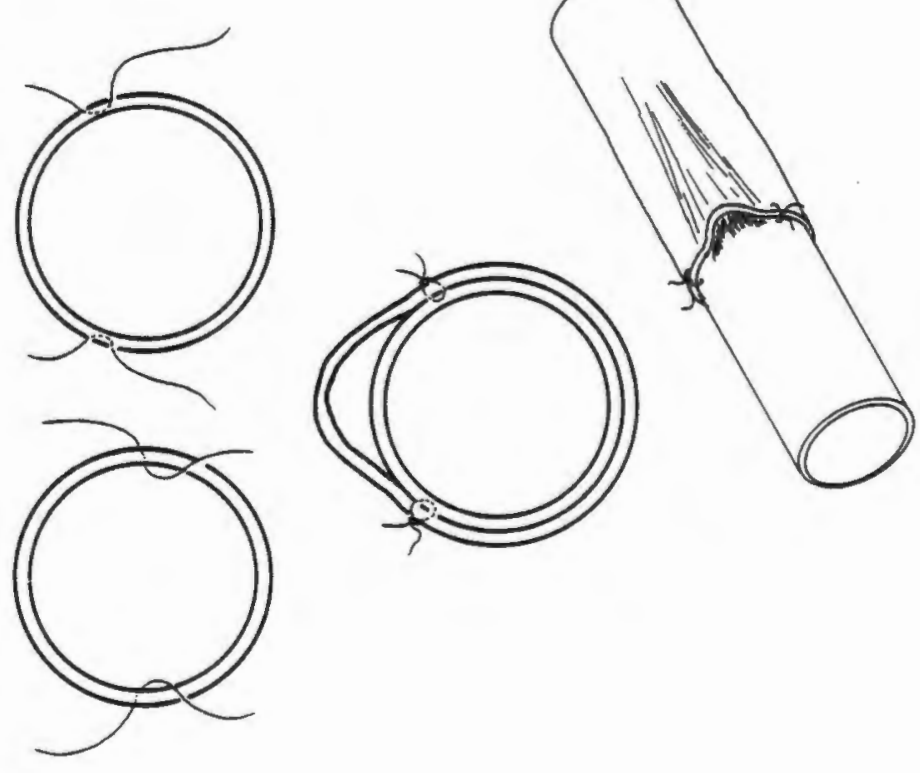

FIGURE 3.5

"Tenting" of RV due to disparate suture separations; - leak channel develops.

When, however, the error was a little more exaggerated as in \#28 \& \#29, the jump-out became inevitable. At the same time difficulties arose with insertion of the FV, as it tended to overshoot and slip past the back of the RV. This complicated the retrieval and insertion of the $F V$, thus increasing the possibility of trauma. 
Chapter 3 - The new EIEA: Development \& Design

In \#30 the mistake was made of placing the sutures closer, together on the FV than on the RV. As expected, this had the effect of slightly "tenting" the RV above the FV, thereby creating a leak channel which could not be corrected by better intermural apposition, but required a fibrin plug to seal it (Fig. 3.5).

In the last two cases the overlap was made shorter than usual. At 1 vessel diameter there were initial difficulties which were resolved, but at 0.7 diameters of overlap problems continued despite extra sutures.

In summary, this exercise revealed the following:

Jump-outs can be unpredictable and may occur in cases where meticulous technique has been applied.

Manual insertion of the $F V$ can sometimes be difficult and may give rise to mechanical damage.

Both vessels must be dilated.

FV adventitia must be trimmed.

RV adventitia need not be trimmed, unless it is obviously excessive.

A $30^{\circ}$ error in suture separation is likely to cause a jump-out.

Errors in the parallelism of suture passes lead to excessive leaking.

\section{THE MEYER TECHNIQUE}

The following description of the steps in this technique was extracted from the publication by Meyer et al (133)(see Fig. 3.6):

* The vessels are held in a double approximating clamp.

* The adventitia is trimmed, more so on the FV than on the RV.

* Both vessels are dilated, with special attention to the RV. 
* Stumps are irrigated with heparinised Ringer's solution.

* The first suture pierces the RV wall about 2 vessel diameters from the cut end, passing into the lumen from outside and emerging through the open end of the RV. It then takes a superficial bite of only the outer layers of the FV, near its cut end. From there it passes back through the open end of the RV and through its wall very near the first pass.

* The suture is cut, leaving a long U-shaped loop of suture material in situ.

* A second similar suture is placed exactly $180^{\circ}$ from the first.

* The clamps are approximated and the sutures are gently tied, thus invaginating the FV into the RV.

The clamps are removed.

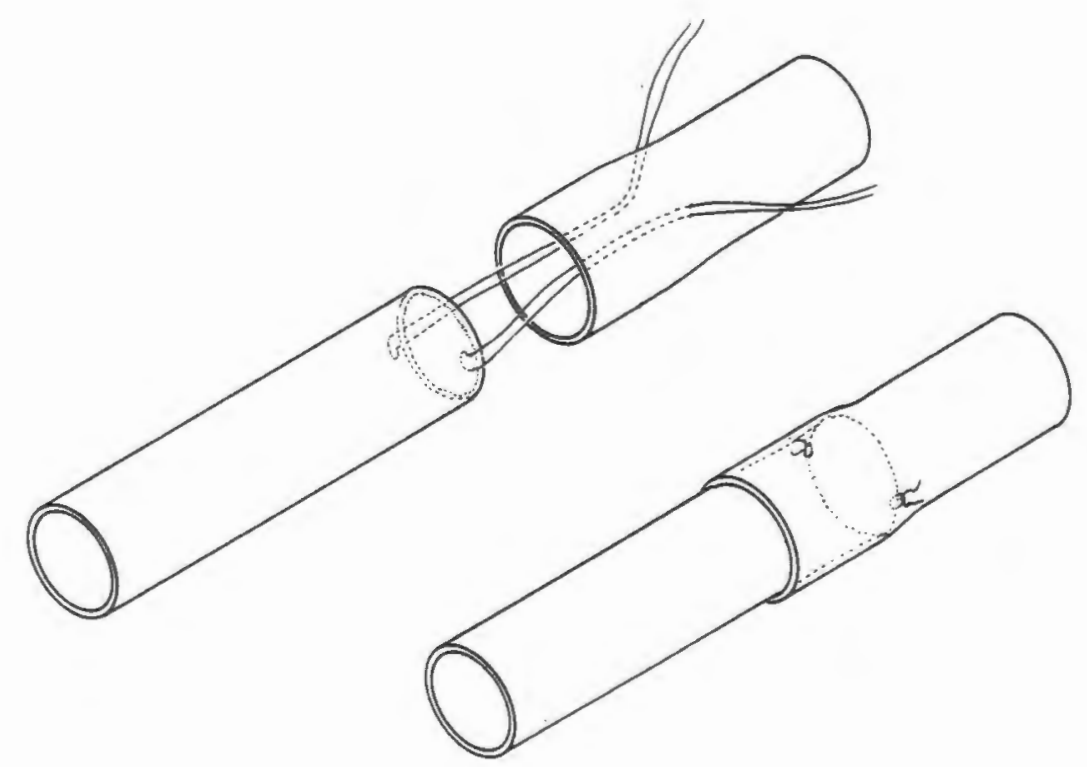

FIGURE 3.6

The method of Meyer et al. 
Twenty anastomoses of this type were executed in the rat femoral artery. Deliberate mistakes were made in the last 8 procedures.

Measurements were made in the same way as those for the Lauritzen technique. Results are displayed in Fig. 3.7.

The following points were noted in the first twelve carefully performed procedures:

There were 3 technical complications (25\%), 2 of which were remediable.

One anastomosis failed completely owing to a back-wall pick-up in one suture pass.

Entwinement of sutures within the lumen could be identified while slipping the FV into the RV. It could then be corrected by removing the entire suture loop and replacing it with a new one, thus taking extra time for the anastomosis.

Even when time was lost by having to replace a suture, the total time taken for the anastomosis was considerably less than would be required for a conventional ETEA.

In cases \#7 to \#12 the RV adventitia was left un-trimmed without any adverse effects.

In the "mistake" procedures :

Case \#13 showed again that it is harmful to the sleeve anastomosis to omit trimming of the $F$ adventitia.

Case \#14 functioned well despite omitting dilation of the FV, but this was probably fortuitous.

The entwinement produced in case \#15 (with some difficulty!) showed that the lumen of the FV could be completely occluded by the tight crossing of the sutures as they are pulled up to invaginate the vessels. 


\begin{tabular}{|c|c|c|c|c|c|c|c|c|c|c|c|c|c|c|}
\hline CASE & $\begin{array}{l}\text { AOVE } \\
\text { TREH } \\
\text { FY }\end{array}$ & $\begin{array}{l}\text { HTIT } \\
\text { HING } \\
\text { RY }\end{array}$ & $\begin{array}{l}\text { VESS } \\
\text { OILA } \\
\text { FY }\end{array}$ & & $\begin{array}{l}\text { FIRST } \\
\text { SUTUR } \\
\text { FY }\end{array}$ & & $\begin{array}{l}\text { SECO } \\
\text { SUTU } \\
\text { FY }\end{array}$ & $\begin{array}{l}\text { OHO } \\
\text { URE } \\
\text { RY }\end{array}$ & $\begin{array}{l}\text { OEGREES } \\
\text { APART }\end{array}$ & $\begin{array}{l}\text { OYER- } \\
\text { LAPP }\end{array}$ & SLIP & $\begin{array}{l}\text { HECH. } \\
\text { OAHAGE }\end{array}$ & $\begin{array}{c}\text { RESULT } \\
\text { IKHEOIATE }\end{array}$ & $\begin{array}{l}\text { RESULT } \\
30 \text { HIN }\end{array}$ \\
\hline $\begin{array}{c}1 \\
2 \\
3 \\
1 \\
5 \\
6 \\
7 \\
8 \\
9 \\
10 \\
11 \\
12\end{array}$ & $\begin{array}{l}y \\
y \\
y \\
y \\
y \\
y \\
y \\
y \\
y \\
y \\
y \\
y\end{array}$ & $\begin{array}{l}Y \\
y \\
y \\
y \\
y \\
y \\
y \\
y \\
y \\
y \\
y \\
y\end{array}$ & $\begin{array}{l}y \\
y \\
y \\
y \\
y \\
y \\
y \\
y \\
y \\
y \\
y \\
y\end{array}$ & $\begin{array}{l}y \\
y \\
y \\
y \\
y \\
y \\
y \\
y \\
y \\
y \\
y \\
y\end{array}$ & $\begin{array}{l}\text { OK } \\
\text { OK } \\
\text { OK } \\
\text { OK } \\
\text { OK } \\
\text { OK } \\
\text { OK } \\
\text { OK } \\
\text { OK } \\
\text { OK } \\
\text { OK } \\
\text { OK }\end{array}$ & $\begin{array}{l}\text { OR } \\
\text { OK } \\
\text { OK } \\
\text { OK } \\
\text { OK } \\
\text { OK } \\
\text { OK } \\
\text { OK } \\
\text { OK } \\
\text { OK } \\
\text { OK } \\
\text { OK }\end{array}$ & $\begin{array}{l}\text { OK } \\
\text { OK } \\
\text { OK } \\
\text { OK } \\
\text { OK } \\
\text { OK } \\
\text { OK } \\
\text { OK } \\
\text { OK } \\
\text { OK } \\
\text { OK } \\
\text { OK }\end{array}$ & $\begin{array}{l}\text { OK } \\
\text { OK } \\
\text { OK } \\
\text { OK } \\
\text { OK } \\
\text { OK } \\
\text { OK } \\
\text { OK } \\
\text { OK } \\
\text { OK } \\
\text { OK } \\
\text { OK }\end{array}$ & $\begin{array}{l}175-185 \\
175-185 \\
170-180 \\
165-175 \\
175-185 \\
165-175 \\
170-180 \\
175-185 \\
170-180 \\
175-185 \\
175-185 \\
165-175\end{array}$ & $\begin{array}{l}2.0 \\
1.8 \\
1.6 \\
2.0 \\
1.6 \\
1.6 \\
2.0 \\
2.2 \\
1.8 \\
1.8 \\
2.0 \\
2.2\end{array}$ & $\begin{array}{l}\text { OK } \\
\text { OK } \\
\text { OK } \\
\text { NOGO } \\
\text { OK } \\
\text { OK } \\
\text { OK } \\
\text { OIfF } \\
\text { OK } \\
\text { OIfF } \\
\text { OK } \\
\text { OK }\end{array}$ & 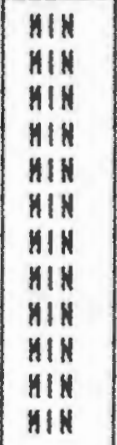 & 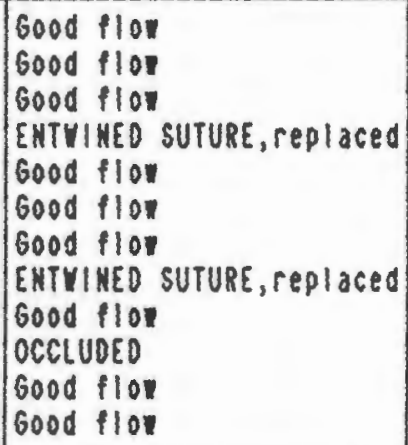 & 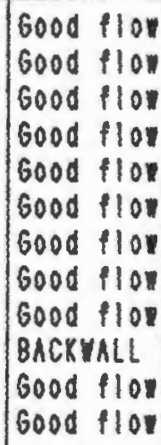 \\
\hline $\begin{array}{l}13 \\
11 \\
15 \\
18 \\
17 \\
18 \\
19 \\
20\end{array}$ & $\begin{array}{l}y \\
y \\
y \\
y \\
y \\
y \\
y \\
y\end{array}$ & $\begin{array}{l}y \\
y \\
y \\
y \\
y \\
y \\
y \\
y\end{array}$ & $\begin{array}{l}y \\
y \\
y \\
y \\
y \\
y \\
y \\
y\end{array}$ & $\begin{array}{l}y \\
y \\
y \\
y \\
y \\
y \\
y \\
y\end{array}$ & $\begin{array}{l}\text { OK } \\
\text { OK } \\
\text { OK } \\
\text { OK } \\
\text { OK } \\
\text { OK } \\
\text { OK }\end{array}$ & $\begin{array}{l}\text { OK } \\
\text { OK } \\
\text { OK } \\
\text { OK } \\
\text { OK } \\
\text { OK } \\
\text { OK }\end{array}$ & $\begin{array}{l}\text { OK } \\
\text { OK } \\
\text { OK } \\
\text { OK } \\
\text { OK } \\
\text { OK } \\
\text { OK } \\
\text { OK }\end{array}$ & $\begin{array}{l}\text { OK } \\
\text { OK } \\
\text { ENT } \\
\text { OK } \\
\text { OK } \\
\text { OK } \\
\text { OK } \\
\text { OK }\end{array}$ & $\left|\begin{array}{l}175-185 \\
170-180 \\
175-185 \\
160-170 \\
110-150 \\
175-185 \\
175-185 \\
175-185\end{array}\right|$ & $\begin{array}{l}1.8 \\
2.0 \\
1.8 \\
1.8 \\
2.0 \\
1.2 \\
1.0 \\
0.8\end{array}$ & $\begin{array}{l}\text { OK } \\
\text { ok } \\
\text { NOGO } \\
\text { OK } \\
\text { OK } \\
\text { OK } \\
\text { OR } \\
\text { OK }\end{array}$ & $\begin{array}{l}\text { HIN } \\
\text { YIN } \\
\text { HIN } \\
\text { HIK } \\
\text { HIN } \\
\text { HIN } \\
\text { HIN } \\
\text { HIN }\end{array}$ & $\begin{array}{l}\text { Good floy } \\
\text { Good flor } \\
\text { ho flor- sut. replaced } \\
\text { Good flor } \\
\text { lEAk } \\
\text { Good flor, sone leak } \\
\text { Good flor, sone leak } \\
\text { LEAK +t+ }\end{array}$ & 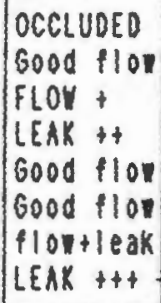 \\
\hline
\end{tabular}

FIGURE 3.7

Meyer's method, results

The angle between sutures seems to be important, as stressed by Meyer (133), in that a deviation from 180 degrees results in excessive leaking. This was demonstrated in cases \#16 \& \#17. There was, however, nothing similar to the jump-out phenomenon.

The anastomosis did not tolerate short overlaps well. In case \#20 with a $0.8 \times$ diameter overlap the leaking could be stopped only by placing additional sutures. The reason for the leaking was the fact that the outer RV could move freely over the surface of the FV and therefore had slipped back to make an even shorter overlap. If the RV was more dilated than the $F V$, it could slip back so far in some places that there was virtually no overlap.

In summary, this exercise revealed the following:

with this technique there are no jump-outs; 
invagination of the $F V$ into the $R V$ is simpler, with less potential for mechanical damage to the intima of the feeding vessel;

it is better, but not essential, to dilate both vessels;

trimming of FV adventitia is essential;

RV adventitia need not be trimmed;

short overlaps are not tolerated by this method; and

the dangers of suture entanglement and backwall pick-up are the worst pitfalls in the execution of this type of EIEA.

\section{THE HYLAND TECHNIQUE}

Hyland et al (87) described a modification of the Lauritzen technique, in which they employed an extra suture U-loop, similar to those used by Meyer, except for the fact that it passed directly through the wall of the FV. This suture (Fig. 3.8) is placed in the middle of the anterior wall of the vessel and is designed to assist with the invagination of the FV and to prevent the "jump-out." The suture is not tied and is removed when the anastomosis has "settled".

I took the liberty of slightly changing the technique to prevent intimal damage in the FV by the additional suture. The suture loop was therefore modified to pass tangentially through the wall of the FV, without penetrating the intimal layer.

The rest of the suture placement and securing technique was exactly the same as that described by Lauritzen. 


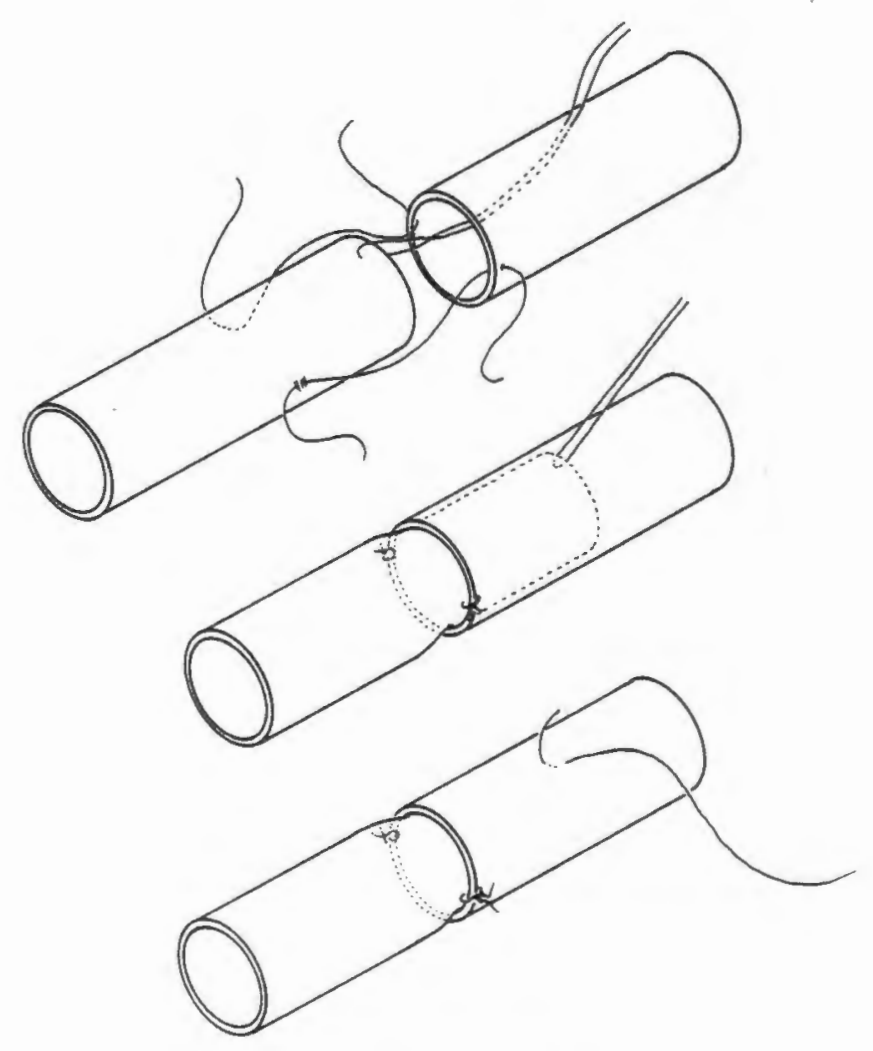

FIGURE 3.8

Hyland's method

Using this method 16 anastomoses were performed in the rat femoral artery. Deliberate mistakes were made in the last 6 procedures. Measurements were made in the same way as those for the other techniques. Results are tabulated below.

The functional results combined with the ease of execution made this technique the most attractive of the three.

Vessel \#8, which became occluded within $30 \mathrm{~min}$ of guide-suture removal, had developed a thrombus arising from the site where the guiding suture's end had been pulled through the wall of the RV during its removal. The FV had withdrawn by one twentieth of a millimetre thus exposing the damaged area.

In cases \#11 to \#13, where deliberate suture separation-angle errors were made, the anastomoses functioned normally. 
This anastomosis also tolerated short overlaps without developing leaks (cases \#14 to \#16).

\begin{tabular}{|c|c|c|c|c|c|c|c|c|c|c|c|c|c|c|c|}
\hline $\begin{array}{l}\text { CASE } \\
\text { No. }\end{array}$ & $\begin{array}{l}\text { AOYE } \\
\text { TRIK } \\
\text { FY }\end{array}$ & $\begin{array}{l}\text { NTIT } \\
\text { HIHG } \\
\text { RY }\end{array}$ & $\begin{array}{l}\text { VESS } \\
\text { DILA } \\
\text { FY }\end{array}$ & $\begin{array}{l}\text { EL } \\
\text { TINE } \\
\text { RY }\end{array}$ & $\begin{array}{l}\text { FIRS } \\
\text { SUTL } \\
\text { FY }\end{array}$ & & $\begin{array}{l}\text { SECO } \\
\text { SUTU } \\
\text { FY }\end{array}$ & & $\begin{array}{l}\text { THIRO } \\
\text { SUTUR } \\
\text { FY }\end{array}$ & $\begin{array}{l}\text { DEGREES } \\
\text { APART }\end{array}$ & $\begin{array}{l}\text { OYERLAP } \\
\text { (mn) }\end{array}$ & SLIP & {$\left[\begin{array}{l}\text { neCH } \\
\text { OAHA }\end{array}\right.$} & RESULT & $\begin{array}{l}\text { RESULT } \\
30 \text { HIN }\end{array}$ \\
\hline 1 & Y & N & $Y$ & $y$ & OK & OK & OK & ok & 0 & & & ok & HIN & Good 1100 & cood flor \\
\hline 2 & $Y$ & r & $Y$ & 1 & OK & OK & ok & ok & & & & OK & HIN & Good floy & $300 d$ flor \\
\hline 3 & $Y$ & N & $Y$ & $y$ & OK & OK & OK & ok & 0 & & & OK & 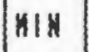 & Good flot & Good flon \\
\hline 1 & $Y$ & N & $Y$ & $Y$ & OK & OK & OK & OK & 0 & & & OK & $n \in N$ & Good flor & 60001101 \\
\hline 5 & $Y$ & N & $Y$ & $Y$ & OK & OK & ox & ok & OK & & & OK & HIN & 6004 floy & 60001100 \\
\hline 6 & $Y$ & N & $y$ & Y & OK & OK & ok & ok & or & & 2 & OK & Hin & $600 d f 101$ & $600 d f 101$ \\
\hline 1 & $Y$ & $N$ & $Y$ & $Y$ & OK & OK & ox & or & or & & 1. & ox & $n \mid N$ & Good flou & 6000 flor \\
\hline 8 & $Y$ & $N$ & y & Y & OK & OK & ox & ox & 0 & & & OK & nin & $600 d$ flor & OCCLUDEO \\
\hline 9 & $Y$ & $n$ & Y & Y & ok & OR & OK & OK & 0 & & & ox & nIN & 6000 flop & 6000 flox \\
\hline 10 & $Y$ & $N$ & Y & Y & OK & OK & OK & ox & OR & & & ox & $n \in N$ & 60001104 & 6000 flor \\
\hline 11 & $Y$ & H & $Y$ & $Y$ & & OK & & ox & & & & 0 & & of Plon & $300 d+108$ \\
\hline 12 & Y & $H$ & $Y$ & $Y$ & 0 & OK & 0 & OK & & & & Ox & N & Good t101 & $600 d 1101$ \\
\hline 13 & $Y$ & $n$ & $Y$ & $Y$ & OK & OK & OK & OK & 0 & & & OK & HIN & Good flor & Good flor \\
\hline 11 & $Y$ & $N$ & Y & $Y$ & OK & OK & OK & OK & 0 & & & OK & H & 60001101 & $6000110 \%$ \\
\hline 15 & $Y$ & $N$ & $Y$ & $Y$ & OK & OK & OK & OK & 0 & & & OK & nIIN & $600 d$ flox & Good flon \\
\hline 16 & $Y$ & $N$ & $Y$ & Y & OK & OK & OK & OK & OK & $175-185$ & 0.6 & oK & HIN & $6000+100$ & 60001100 \\
\hline
\end{tabular}

FIGURE 3.8(b)

Results when using the method of Hyland et al.

In summary, the Hyland technique exercise showed me the following:

the "jump-out" had been overcome;

insertion of the FV into the RV was easier and was less liable to damage the FV intima mechanically;

although it did not happen in my study, I saw the potential for the mistake of including the backwall in the guiding suture. The frequency of this occurrence should be the same as that in the Meyer technique (133);

the fact that there is only one deep intraluminal guiding suture eliminates the possibility of intraluminal entanglement, as seen in the Meyer technique (133); 
the additional guiding suture makes the procedure take longer initially than the Lauritzen technique (112), but it makes insertion of the FV into the RV consistently easier and may therefore, on average, lead to overall timesaving; and

trauma caused by the additional suture is counterbalanced by the elimination of all further injury during invagination. Damage by the modifying suture, when compared to the potential trauma by virtue of the manipulations required in the Lauritzen technique, is virtually negligible.

\section{ANALYSIS OF RESULTS}

The following were the most important points derived from the study:

1. It is not necessary for the success of an EIEA to trim the adventitia of the RV. This could be an added advantage of the EIEA, when considered in the light of the findings by Eisenhardt et al (59), who showed that removal of the adventitial layer with its vasa vasorum causes necrosis of the media and aneurysm formation in the same area.

2. The critical accuracy required in separating the sutures by 180 degrees can be a definite problem in both the Meyer and Lauritzen techniques.

3. The long, looped sutures of Meyer and Hyland simplify invagination of the FV into the RV and they prevent the jump-out, but they have certain disadvantages:

3.1 when two deep loops are used, there is a danger of intraluminal entwinement;

3.2 during the placement of any deep, looped suture, the backwall may be picked up in a needle pass, thus occluding the vessel; and 
3.3 if the suture is removed, as in the Hyland technique, it may leave an exposed area of intimal damage which is conducive to thrombus formation.

4. All additional sutures and manipulations take extra time. The requirement for these procedures is usually not anticipated. This leads to frustration, thereby affecting the surgeon's performance in the remaining steps of the operation.

\section{DESIGNING A NEW METHOD}

\section{APPROACH}

The precise nature of microsurgery makes it highly desirable to eliminate as many potential problems as possible from a procedure. The same principle would undoubtedly apply to all forms of surgery, but it is especially valid in the field of microsurgery, because in this milieu all the dexterity and thought processes of the surgeon are concentrated upon a very small area. This area, the operating field in which the entire process of identifying the vessel ends, dissecting them free, debriding them and then effecting an anastomosis must take place, may be as little as $10 \mathrm{~mm}$ in diameter. The surgeon often tends to feel like an outsider looking in on the proceedings. When matters do not go according to plan, it is difficult to communicate problems to those around one, i.e. to those who cannot see the cause of the dilemma. One may even find it difficult to associate or compare the difficulties under the microscope with those in "one's own world".

Very often the responses to the problems are entirely inappropriate, e.g:

When one has difficulty in picking up a thread with the forceps, one naturally tends to grip the forceps more tightly, thus decreasing the fine motor control of one's hand, increasing tremor, and possibly even decreasing the gripping capability of the forceps by shifting the contact area proximally (Fig. 3.9). 


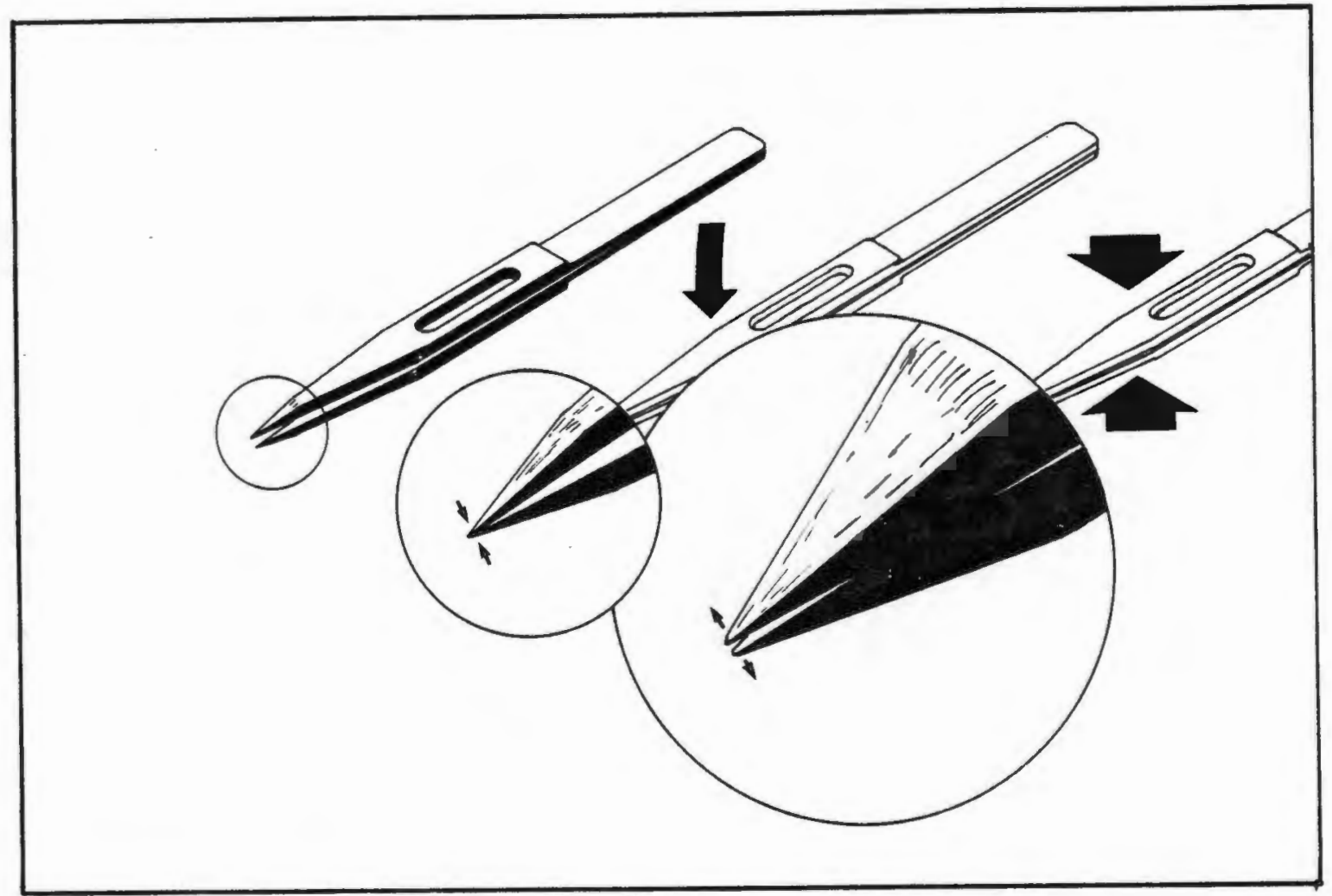

FIGURE 3.9

Effect of gripping forceps too hard; contact area shifted back and tips open thus decreasing effectiveness of grip.

Problems which cannot be communicated to assistants and for which their own natural responses are inappropriate, can lead to tremendous frustration in the minds of the surgeons, thus making their task even more difficult as a result of normal physiological responses to stress.

The vicious cycie of problem - inappropriate response - worse problem is most easily avoided by very careful and deliberate planning by the surgeon of every single movement which is to be carried out, based on a thorough knowledge of the procedure which is being followed. This holds true provided that the procedure itself is not riddled with inherent faults or disaster potentials.

With the goal of minimising the disaster potential of the EIEA, I set out on a problem-orientated basis. If I could find feasible solutions to the problems I had discovered, I believed that they would lead to a method which would be consistently more successful in the hands of a larger number of surgeons. 


\section{PROBLEM-ORIENTATED PLANNING}

Attempts were made to solve each current or potential problem individually. In some cases the solution to one problem also provided an indirect solution to others.

\section{JUMP-OUTS}

A jump-out, or a severe leak, occurs in an EIEA when any part of the end of one vessel slips back towards the end of the other vessel to the extent that overlap is partially or completely lost.

The Hyland technique (87) prevents the jump-out from occurring. It also prevents the slipping back of the RV as seen in the Meyer technique (133).

The reason for these successes by the Hyland method lies in the fact that the guiding suture, before its removal, holds the end of the FV fixed to the wall of the RV and at the same time the "Lauritzen" sutures are holding the end of the RV fixed to the wall of the FV (Fig. 3.8). Since the end of each vessel is fixed to the wall of the other vessel, it is impossible for either end to move back to the extent that overlap would be lost, unless the initial overlap is extremely short.

It is conceivable that, when anticoagulants are used, as is usually the case in clinical vascular surgery (54), the FV may not have become adherent to the inner surface of the RV by the time that Hyland's guiding suture is removed. The anastomosis at that stage effectively becomes a Lauritzen anastomosis and may occasionally suffer a jump-out.

I would prefer to see the guiding suture tied and left securely in place, thus definitely preventing the jump-out.

\section{INVAGINATION}

There are only two basic ways in which one tubular structure may be placed within the lumen of another tubular structure: it may be pushed in or it may be pulled in.

Pushing can be done by a variety of mechanical and physical means, such as direct manipulation with instruments or the use of jets of fluid or gas. 
"Pulling" implies that the inner tube is "led" or guided into the outer tube and it can only be done by a force applied from within the outer tube. The application of the force can be achieved by direct mechanical means or by other means, such as suction, magnetism or gravity.

The decision whether to push or to pull should depend upon the type of material of which the tubes are made. Rigid tubes are easily telescoped by pushing one into the other, but soft or flaccid tubes are telescoped more successfully when the inner tube is pulled into position while the end of the outer tube is either fixed, or is pulled in the opposite direction.

Pushing a soft, pliable tube into position inside the other tube is almost impossible if the tube is not grasped at its very end, which essentially means that it is being "pulled" in. This kind of "pulling" has the inherent disadvantage that the "puller" has to fit into the lumen of the outer tube alongside the tube which is being pulled into position. When the telescoping action has been completed, the "puller" must be withdrawn from its position between the two tube-walls and may by friction or other adhesive forces bring out the inner tube with it. It follows that when either of the two tubes is of a delicate or vulnerabie constitution, such a manoeuvre is undesirable.

There is little doubt that a flaccid inner tube can be more effectively and accurately pulled into place by a force applied from within the lumen of the outer vessel.

In order to apply the pulling principle by means of other than electromagnetic or gravitational forces, access must be gained to the lumen of the outer tube and when this tube is long, or when it is closed at the other end, such access must needs be through the wall of the outer tube.

Since blood vessels are soft tubes with ends which are effectively closed, I shall pursue this line of argument.

Having decided to breach the wall of the outer tube to gain access to the lumen, it was necessary to explore the different instruments and transmitters of tensile force which could be used to carry out the pulling action within the lumen of the outer tube.

Consideration firstly had to be given to the fact that, if mechanical means were used and disruption of the wall of the outer tube had to be limited, it would probably be best to keep the "motor" outside the tube and to use a tensile-force transmitter, such as a rod or towline, passing through a small hole in the wall of the tube, to apply the force. Secondly, it was not only the force 
transmitter, but also the fixator (i.e. the device which gave attachment to the inner tube), which had to pass through the wall of the outer tube.

The fixator could be a hook, a catch, a magnet, a suction cup or a mere passage of a towline passing through a hole in the wall of the inner tube. If the tubes are blood vessels, the fixator would have to be :

as small as possible, to avoid damaging the wall of the outer vessel excessively;

removed or hidden at the completion of the invagination, to avoid contact with the blood stream; and

atraumatic in its method of fixation to the inner vessel.

Of the possible fixators, the only two that would be both suitable and widely available to surgeons are :

1. A suction cup device.

2. A fixed towline.

\section{Suction cup:}

A fixator of this type can be made by passing a very thin cannula through the wall of the outer vessel and out through the open end of the lumen. The tip of the cannula can then be placed against the outside of the wall of the inner vessel near its cut end. When suction is applied to the other end of the cannula, it will attach itself to the wall of the inner vessel. By drawing back the cannula while maintaining suction, the inner vessel will be drawn into the outer vessel up to the level of the hole (Fig. 3.10).

When invagination is complete, the suction could be disconnected from the back end of the cannula, thus releasing the inner vessel.

However, such a system would have the disadvantages of:

1. being relatively bulky, thus making a relatively big hole in the sidewall of the outer vessel; 
2. poor control over damage to the wall of the inner vessel by the sucking action of the cannula tip; and

3. possible damage to the intima of the outer vessel by the tip of the cannula.

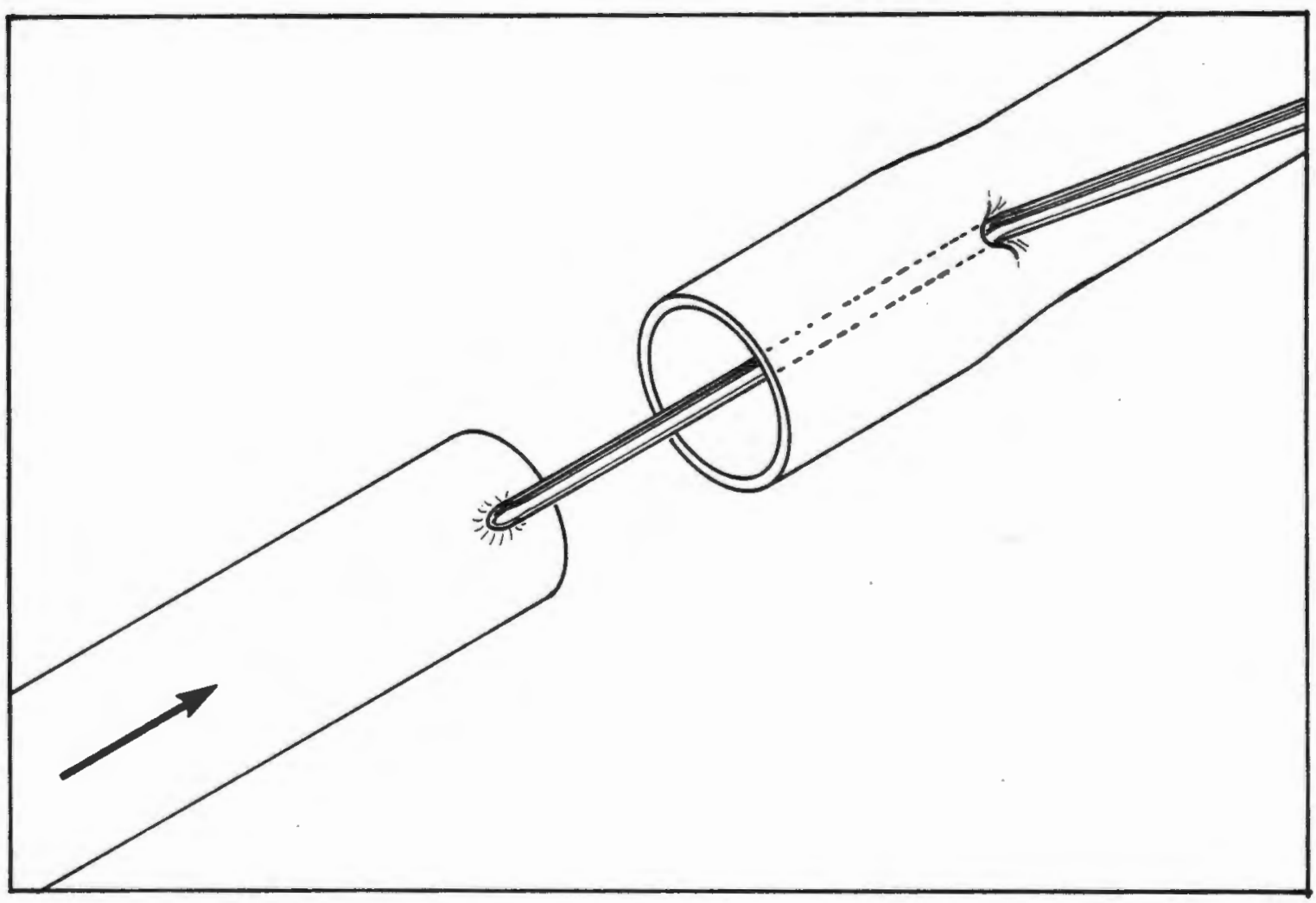

FIGURE 3.10

The suction-cup invaginator.

\section{Towline}

A towline in the form of a suture is always available to the surgeon. The fixation methods for attaching the towline to the inner vessel can vary and should therefore be examined more closely.

Possible methods of fixation are:(Fig. 3.11)

1. pass a suture through the wall of the inner vessel and tie it to itself (Fig. 3.11.1); 
2. pass a suture through the outer layers of the inner vessel's wall and tie it to itself [Fig. 3.11.2];

3. glue the end of the suture to the outside of the inner vessel [Fig. 3.11.3];

4. pass the suture through the wall of the inner vessel, then bring it back through the lumen of the outer vessel and pull on both ends [Fig. 3.11.4]; and

5. pass the suture through the outer layers of the wall of the inner vessel, then bring it back through the lumen of the outer vessel and pull on both ends of the suture [Fig. 3.11.5];

Method 1:

Advantage :

Disadvantage:

Advantage :

Disadvantage: only one small hole in side of outer vessel.

suture and knot must remain within lumen after invagination

\section{Method 2:}

only one small hole in side of outer vessel.

\section{Method 3:}

Advantages:

1. only one small hole in side of outer vessel;

2. no knot between vesse/ wall and

3. remaining suture material hidden between walls of the vessels. 


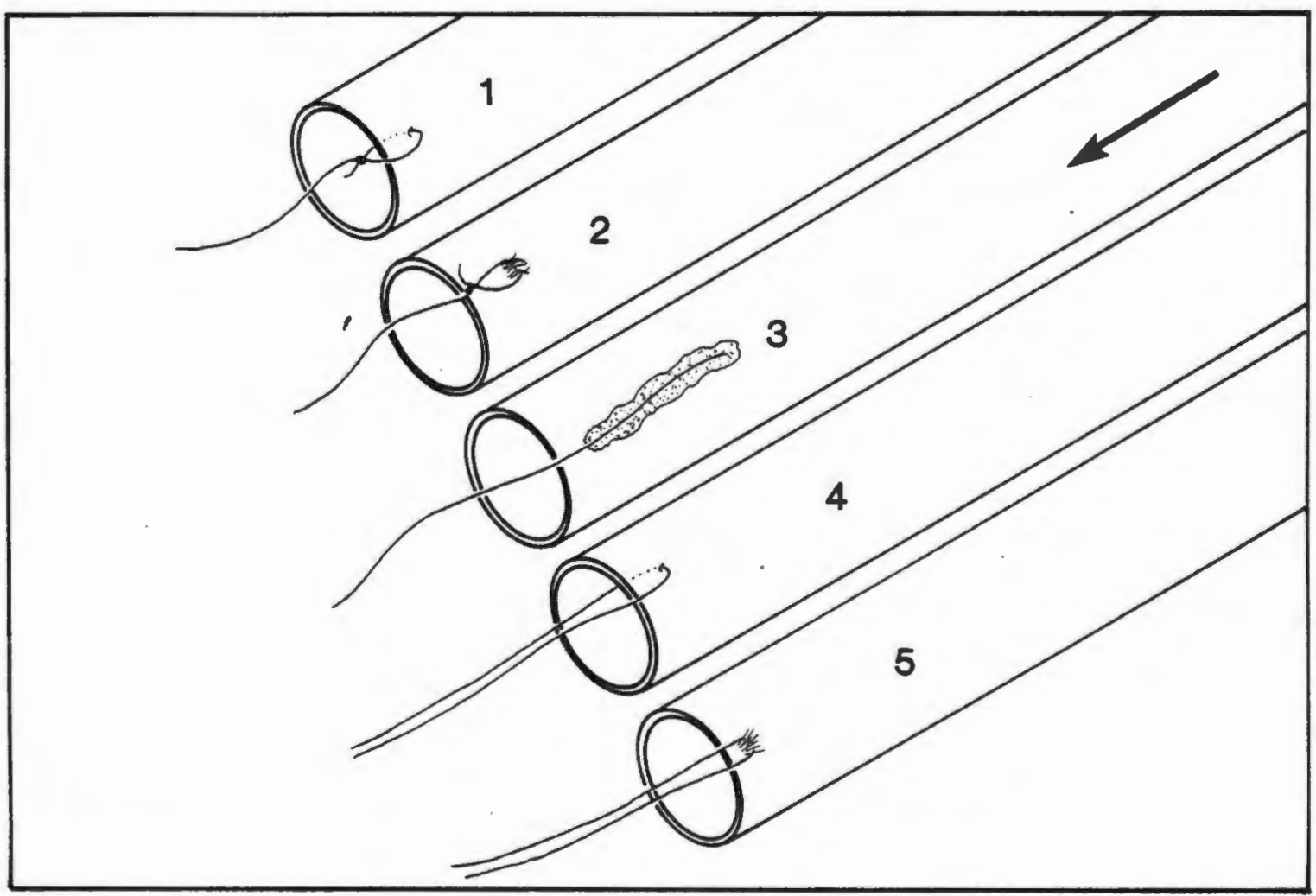

FIGURE 3.11

Methods of towline fixation; $\mathrm{f} 1$ - $\mathrm{f5}$

Method 4: (ct. original Hyland technique)

Advantages :

1. towline can be removed after invagination;

2. double strength towline; and

3. uses standard materials.

Disadvantages: 1. two holes in outer vessel's wall;

2. hole in inner vessel's intima and 
3. three needle passes required.

Method 5: (cf. Meyer technique).

Advantages: $\quad$ 1. towline can be removed after invagination or it can be tied over the wall of the outer vessel, thus hiding it completely away from the blood stream;

2. double strength towline; and

3. uses standard materials

Disadvantages

1. two holes in outer vessel's wall (note: if the suture is tied outside the outer vessel, the holes in the outer vessel wall will be hidden from the blood stream by the infer vessel's wall); and

2. three needle passes required.

With development and sophistication of surgical adhesives, Method 3 may become the method of choice, but under the prevailing circumstances, looking only at the mechanism for invagination, I agreed with Meyer et al (133) in their choice of Method 5. Of all the possible ways 1 considered for effecting telescoping of the vessels, this method had the fewest obvious disadvantages.

Having established the need for a long looped suture, placed deep in the lumen of the outer vessel (RV), to provide the mechanism for invaginating the vessels, I then examined the problems which could result from this type of suture.

\section{ENTWINEMENT}

Entwinement can take place within the loop itself. This is not true entwinement, but rather a twisting of the suture about itself. This twisting can be avoided by keeping the suture material straight and keeping the loop flat while placing the suture (Fig. 3.12). True entwinement occurs when two or more sutures are present in the area, i.e. when, as in the methods of Murphy (135) and Meyer (133), two or more deep loops are used. 


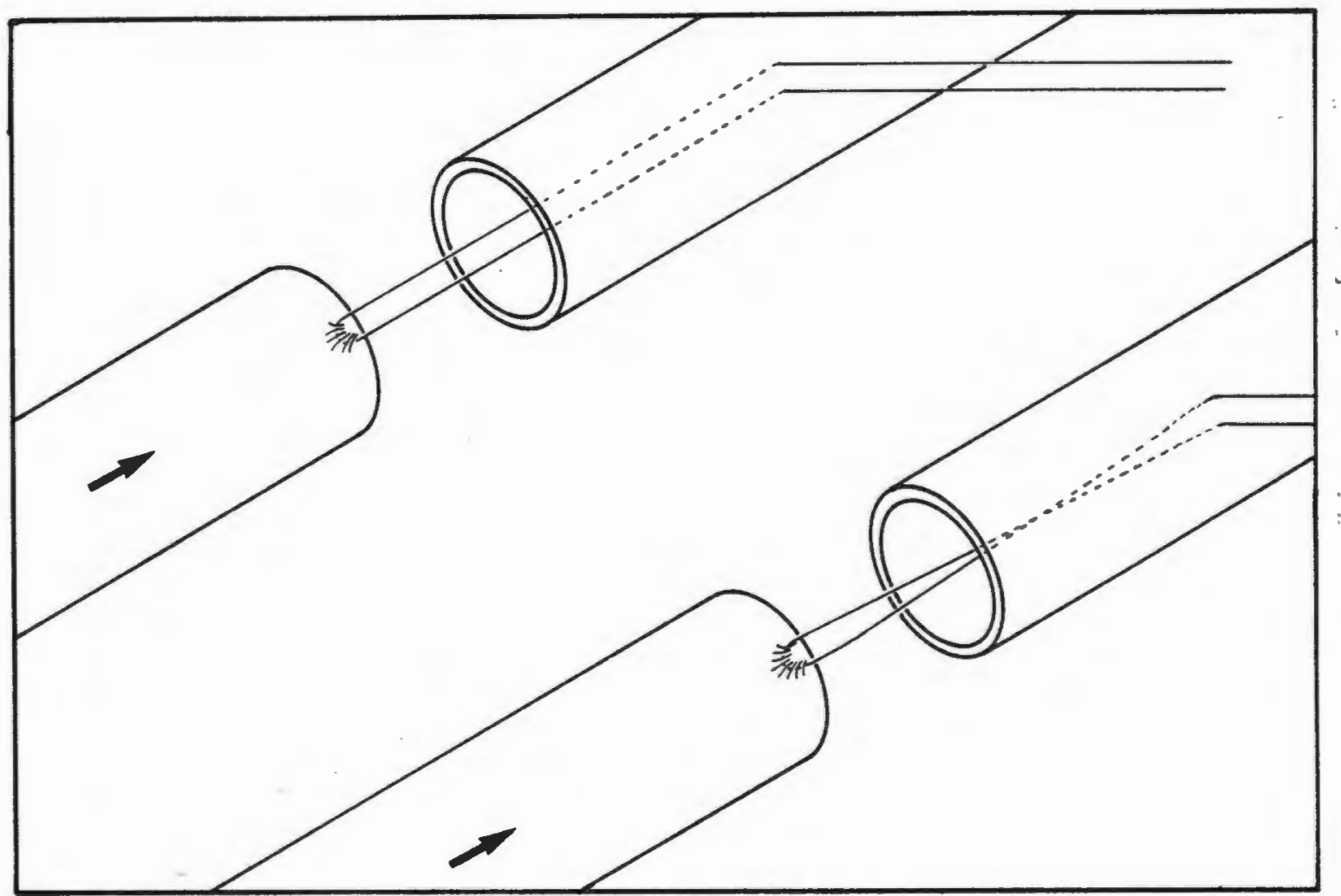

FIGURE 3.12

Flat loop (good) vs Twisted loop (bad)

The space within the lumen of the microvessel is less than $1 \mathrm{~mm}$ in diameter. This means that, even if the completed first loop was lying unusually flush against the inside of the outer vessel, the suture needle, which is placing the next loop, would have to stray less than $1 \mathrm{~mm}$ out of its path to pick up one of the strands of the first loop.

To compound the problem, the pick-up can take place at a position inside the vessel where neither the point of the needle nor the strands of the other loop are visible to the surgeon.

As we have already seen, entwinement of the two loops causes difficulty with invagination. It may also cause complete occlusion of the vessel. The only way to remedy the situation when it has occurred, is to remove one of the sutures completely and to replace it (with the possibility of creating a second entwinement in the process!).

The likelihood of creating an entwinement must logically increase with the number of sutures lying within the lumen. 
The obvious way to avoid entwinement is therefore to use only one deep looped suture. This was done successfully in the modified Hyland technique.

\section{BACKWALL PICK-UP}

While placing the deep looped suture, there is a danger of incorporating the backwall of the RV. This applies especially to the first pass, from outside to inside, for three reasons:

1. The vessel, being empty, is easily collapsed so that the front and back walls lie in close apposition.

2. The needle, while passing through the front wall, exerts pressure on it thus pushing it against the backwall.

3. The first needle pass is 1 to 2 vessel diameters away from the cut end of the vessel and it is therefore close to the clamp which is compressing the front and back walls together to prevent bleeding.

For any one of these reasons, the backwall may be accidentally incorporated in the suture pass opposite its point of emergence from the intima of the front wall and far from the end of the vessel. Such a backwall "pick-up" is difficult to detect before clamp release reveals occlusion of the vessel. The consequences may be disastrous, as the whole anastomosis will have to be repeated and such a procedure has a much higher potential for thrombosis than a "first time" anastomosis. This is due to (a) endothelial damage and platelet and fibrin deposition during the first anastomosis and its clamp release period, and (b) clamp trauma during re-application.

To prevent backwall incorporation the following measures were considered:

1. Only using sutures passing from inside to outside the RV lumen.

2. Moving the clamp further up the vessel, away from the cut end.

3. Placing a protector between the front and back wall while passing the needle.

The first option could be used if double-armed micro-sutures were freely available, or if the glued-on towline method could be employed for invagination.

The second option requires approximation clamps with longer bars, as well as more extensive dissection of the vessels, thus making it an unattractive solution. 
Placing a protector between the front and back walls seemed possible, provided that the protector itself did not cause endothelial damage. An instrument which would protect the endothelium of the RV from being damaged by the needle or being incorporated in the suture pass, would ideally have the following characteristics:

1. It should have a very smooth, polished finish, with no sharp or abrasive edges or surfaces.

2. It must be small enough to fit loosely into the lumen of the blood vessel.

3. It should protect the entire inner surface of the RV, not merely the backwall.

4. It should also act as a counterpressure device in the process of passing the needle through the front wall of the vessel.

Two possible solutions were considered.

(a) A rod of relatively soft material, which could be polished and which had a blunt, rounded or bullet-nosed end, which could be placed in the lumen of the vessel. The suture could then be passed through the vessel wall and directly into the soft material of the rod. The point of the needle would thus be covered and could safely continue its passage through the lumen while embedded in the rod.

The rods were made from $20 \mathrm{~mm}$ pieces of No.1 monofilament nylon suture [Ethilon], which has a diameter of approximately $0.5 \mathrm{~mm}$. Their ends were rounded by bringing them into the vicinity of an open gas flame and they were then polished by means of a slow buffing-wheel and jeweler's rouge under a dissecting microscope.

(b) A hollow metal tube with the edges of its open end rounded and polished. The needle could then be passed into this tube, which would protect the entire inner surface of the vessel wall. Advantages could be 
gained by cutting the end of the tube obliquely, as the rounded edges would then form an oval counterpressure ring similar to the one developed by Acland (5), with the added feature of complete backwall protection (Fig. 3.13). The oblique end would also facilitate insertion of the tube into a collapsed vessel.

Prototypes were fashioned from 30-gauge hypodermic needles by a process of grinding and polishing, which was developed by a combination of planning and "trial and error" methods. When polishing steps were not carried out in the correct sequence, parts of the edge could actually be sharpened while attempts were being made to smooth other areas.

The polished cannulae obtained by this method not only functioned well as planned, but were also found to have a number of other uses, which made them indispensable on my instrument tray. Appendix A provides a full description of the polished-needle cannula and its uses.

Both of these backwall protectors functioned well, but the cannula was more versatile and it therefore became my instrument of choice.

\section{EXTRA SUTURES}

When additional sutures are employed in the EIEA, some of the advantages of this concept may be lost. The added sutures take extra time and they may restrict the coaptative movements of the blood vessels, thus leading to suboptimal flow patterns in the vicinity of the anastomosis $(114,113)$.

In using the previously described EIEA techniques, I have never experienced the need to use additional sutures to improve the tensile strength of an EIEA. I have, however, frequently found the need to place one or two extra sutures in an attempt to stabilise a "jump-out" or to control a severe leak due to slipping back of the RV's end. It follows that a method which would minimise such episodes of compromisation, would also remove the need for additional sutures. 


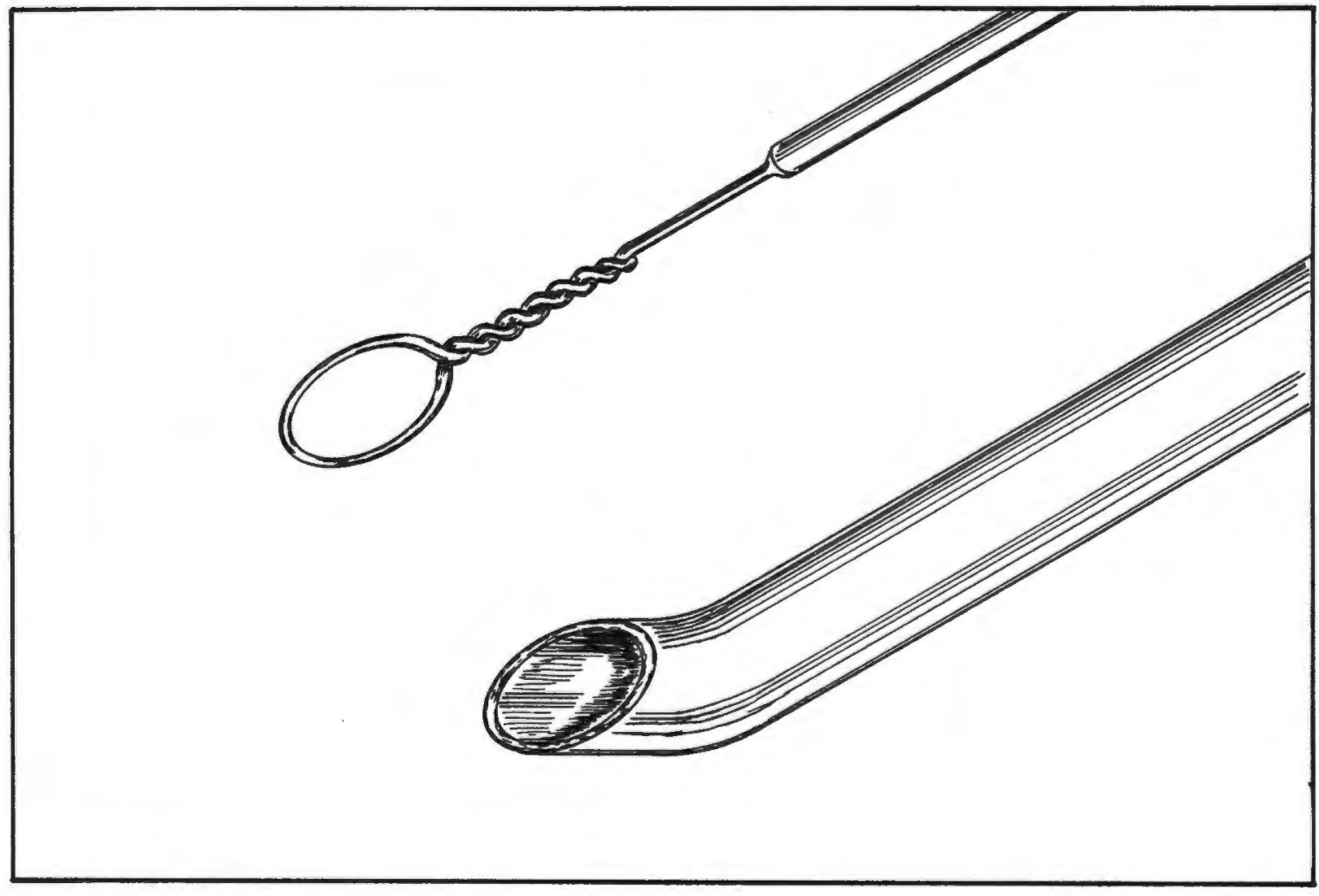

FIGURE 3.13

Acland's counterpressure ring device compared with the polished-needle cannula

\section{SUMMARY}

The following points emerged from the problem-orientated analysis:

1. To prevent the jump-out, the end of each vessel stump should be fixed to the other vessel's wall.

2. To facilitate invagination, a deep looped suture should be used.

3. To prevent intraluminal entwinement of sutures only one deep looped suture should be employed.

4. Incorporation of the backwall during placement of the deep loop can be prevented by means of the polished-needle cannula.

5. The use of more than two sutures in the anastomosis is undesirable and should be unnecessary if jump-outs and severe leaks can be avoided. 


\section{THE ASYMMETRICAL SUTURE CONCEPT}

By taking an overview of the results and of logical conclusions reached by that stage, I found that a new method for the EIEA had, apart from the finishing touches, presented itself.

If only two sutures were to be used and the end of each stump was to be fixed to the sidewall of the other vessel, then it would require a suture placement which was not symmetrical about the axis of the vessel (Fig. 3.14). The suture used to fix the end of the FV to the wall of the RV had to be a deep looped suture, placed with the aid of the polished-needle cannula.

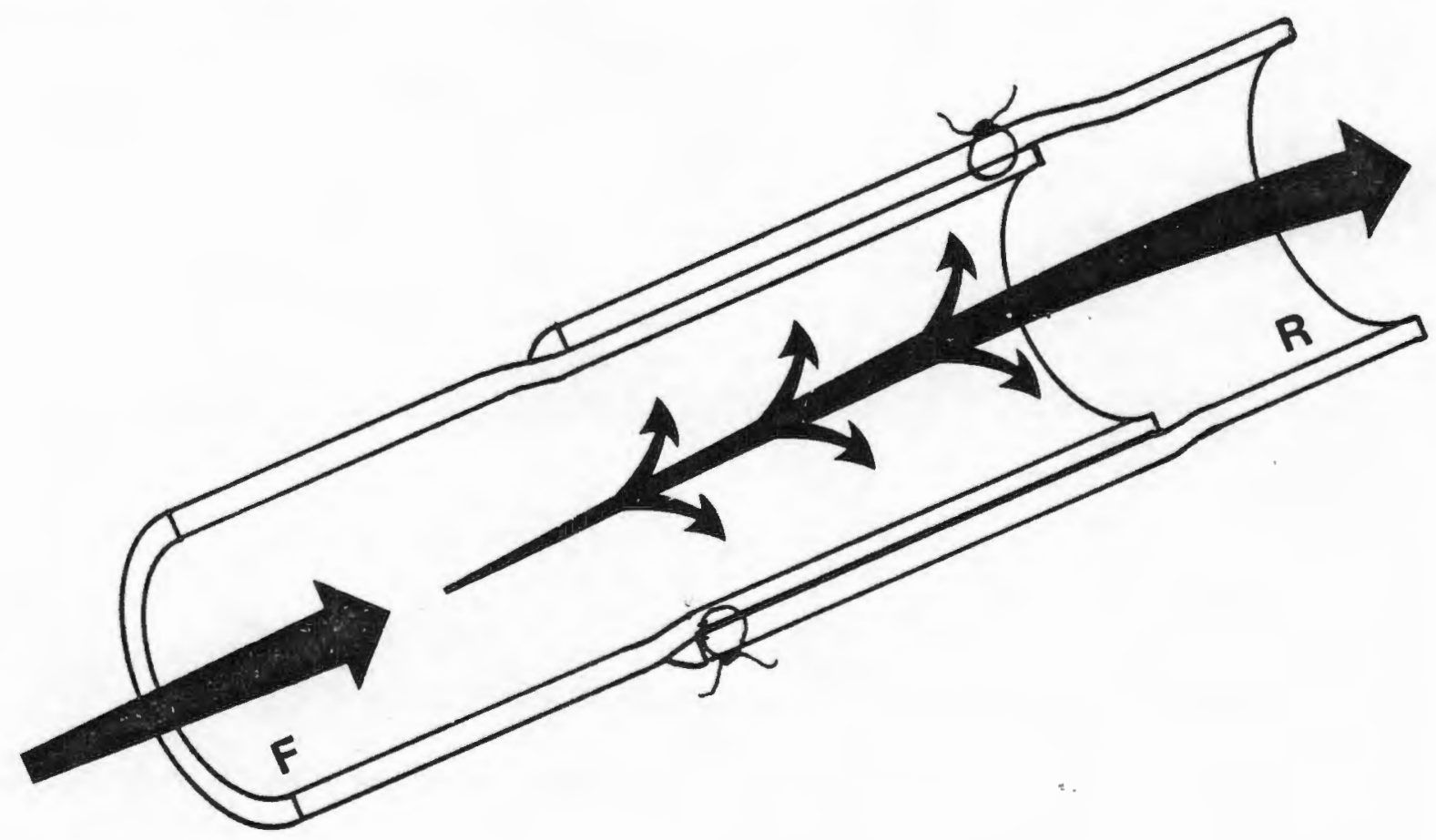

FIGURE 3.14

The asymmetrical suture concept.

The exact configuration of the other suture required to fix the end of the RV to the side of the FV, has until now been neglected. This suture would also act as an anchor or provide countertraction during the process of invagination. If it was to act as an anchor, it would have to be tied before invagination of the vessels. This would mean that the vessel ends would 


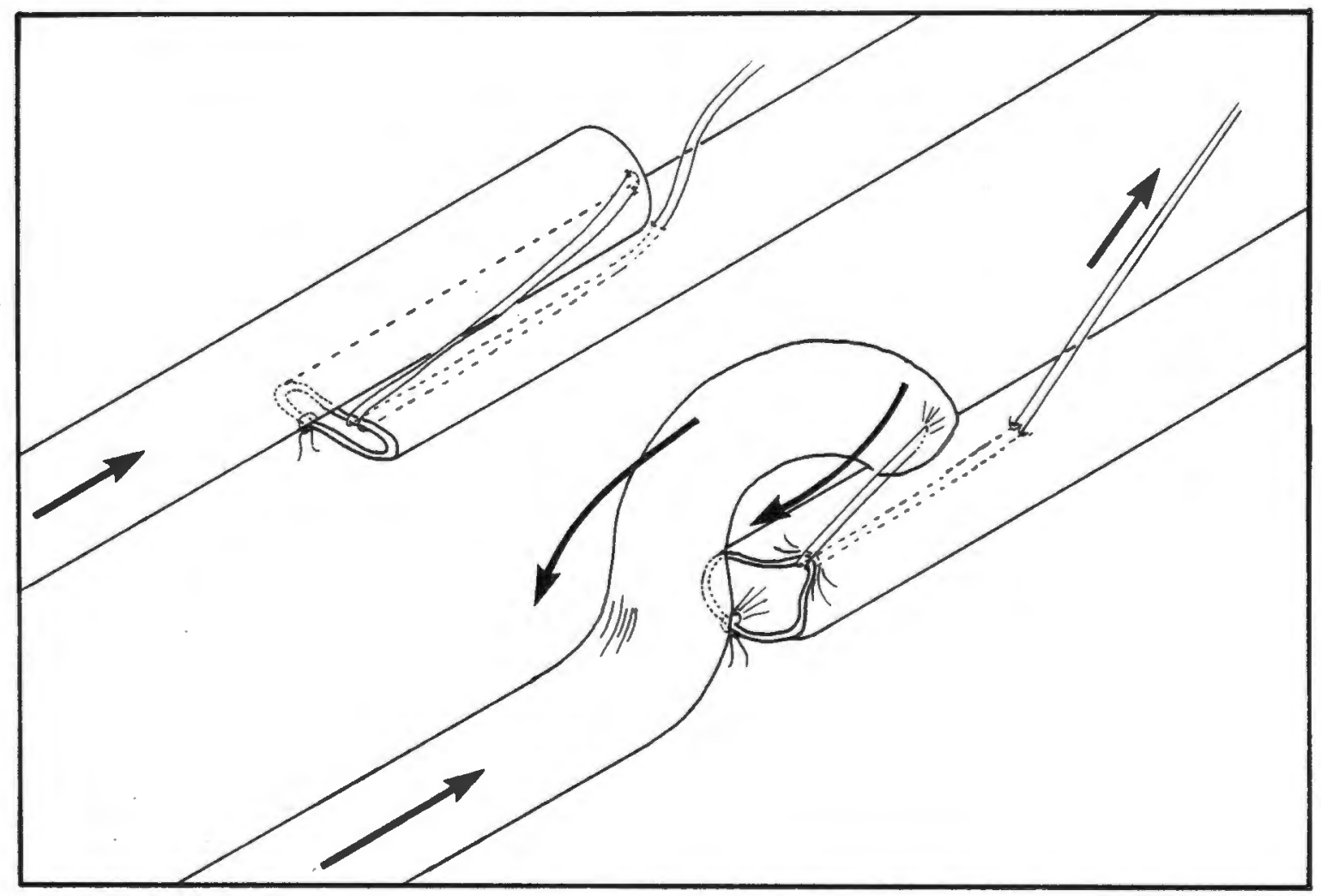

FIGURE 3.15

Showing the movement of the FV's end when the 2 nd suture is tied before invagination.

initially be externally overlapped. In order to move into the RV lumen from this overlapped position, the end of the FV would, in relation to the RV, first retreat and then advance into the lumen of the RV. The retreat phase would be motivated by the deep looped suture dragging back the end of the FV towards the end of the RV around the edge of which the suture is hitched (Fig. 3.15). This movement would cause kinking of the FV and a sawing action to the edge of the $\mathrm{RV}$ and would thus be potentially harmful to both vessels. If the second suture was to act as a countertraction it could do so in either a dynamic or a static fashion.

A static counter traction would behave in much the same way as an anchor, but dynamic counter traction could mean that the RV would be sliding over the FV, while the FV was sliding into the RV. In such a situation neither of the vessels would be kinked during invagination. Three different suture configurations, capable of providing dynamic counter traction, were considered (Fig. 3.16).

For the suture to move the RV over the FV, the suture material must move through the tissue of one or both of the vessel walls. Any movement of a suture through tissue may have a cutting or sawing effect, which is potentially harmful to that tissue. Considering the fact that the FV was the 
vessel whose intima was exposed to the blood stream all the way to the end of the stump and that the RV at the level of this suture would be completely lined by the FV, I decided for the following reasons to use the 3 rd suture configuration (Fig. 3.16c):

1. There would be no relative movement between the suture and the wall of the FV during invagination.

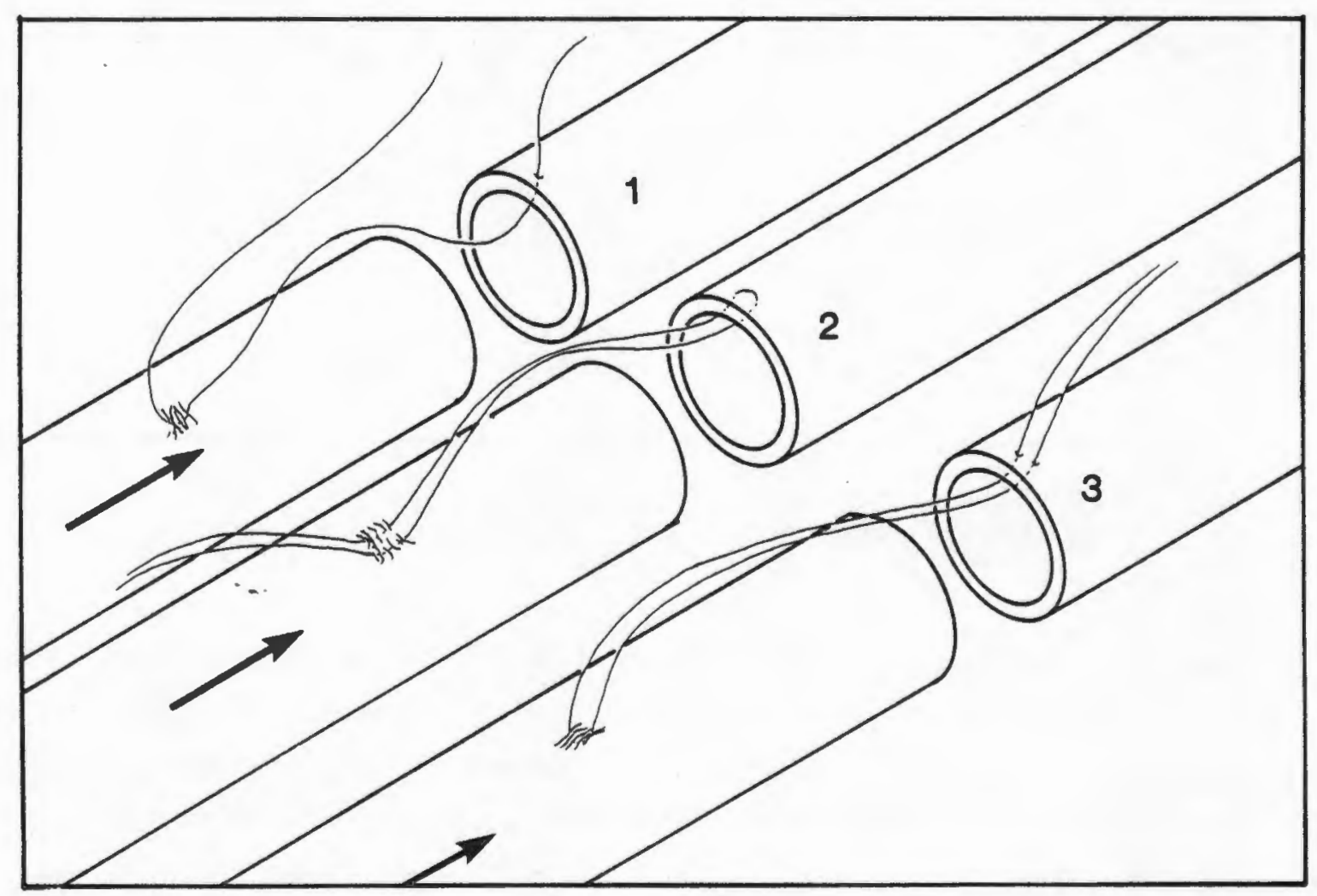

FIGURE 3.16

Three different suture configurations capable of providing dynamic counter traction; were considered

2. All relative motion between suture and tissue would take place at the two points where the suture passes through the wall of the RV. Here damage would have little effect on the viability of the anastomosis.

3. The relative movement would take place at two adjacent sites in the RV's wall, thus halving the frictional or cutting force at each point. 
4. The relative rate of movement of the vessels during the invagination procedure would be similar to that obtained with the deep looped suture which controlled the other half of the invagination process.

In the first configuration (Fig. 3.16a) the suture would move through both vessels' wall-tissues and the relative rate of motion would differ from that caused by the deep looped suture during invagination. The second option (Fig. 3.16b) would cause all the movement of the suture to take place through the tissues of the FV's wall.

Having decided upon the configuration of each of the two sutures involved in the anastomosis, I completed the "drawing board" design of the new EIEA (Fig. 3.17).

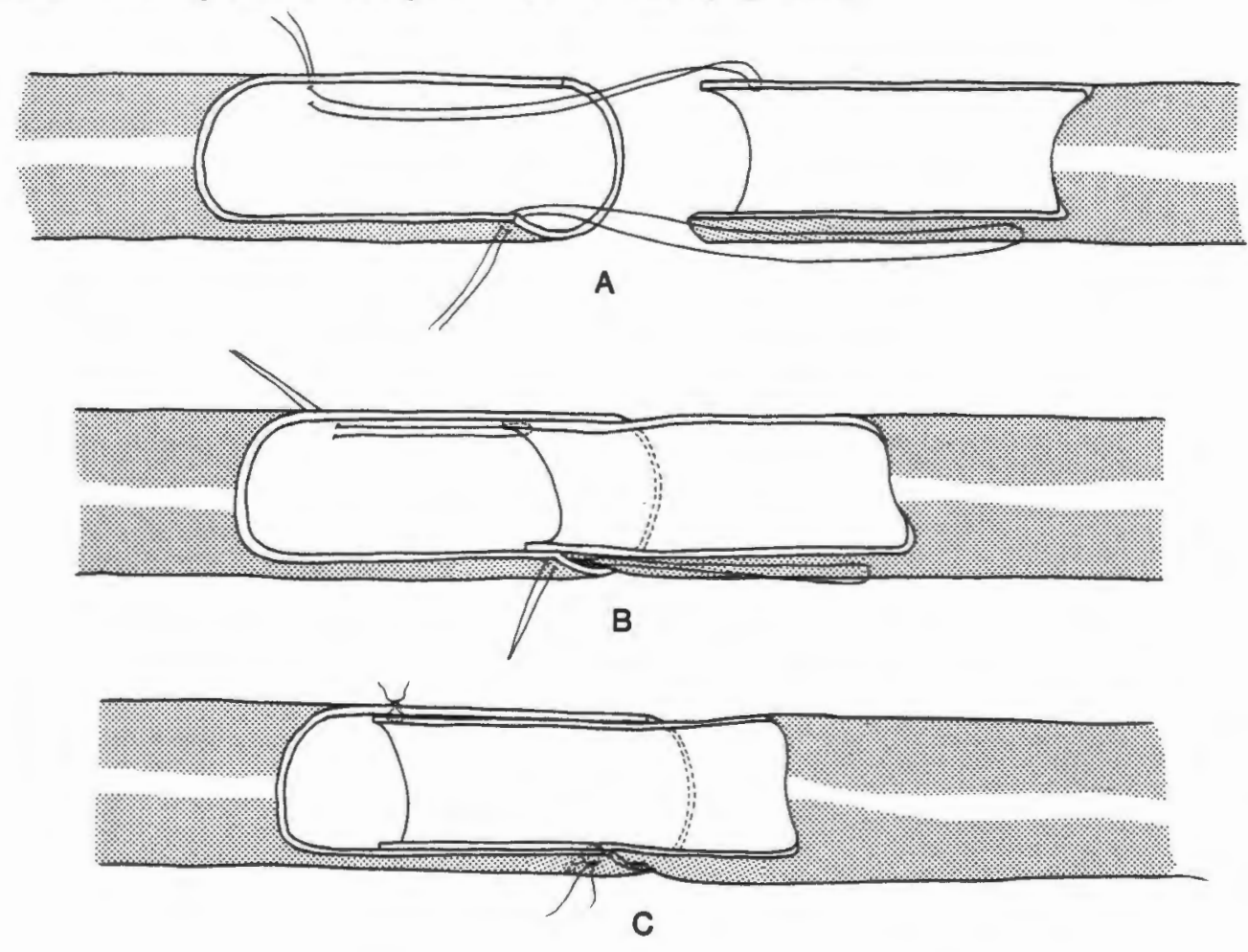

FIGURE 3.17

"Drawing board" design of the new EIEA

\section{Theoretical Advantages}

The new EIEA would theoretically possess the following advantages over previous EIEAs:

1. No jump-outs. 
2. Suture-controlled automatic invagination with no need for additional manipulations.

3. No entwinement of sutures in the RV lumen.

4. Complete backwall protection during placement of the deep looped suture.

5. Only one suture on any single transverse section of the vessel (Fig. 3.18), leading to:

a) little scarring which may cause stenosis.

b) more scope for coaptative movement between vessels' walls; and

c) little interference with blood supply to vessel wall via vasa vasorum.

6. No intraluminal suture material and no damage to the intimal surface. (This is also true in the Lauritzen and Meyer techniques).

7. Potential for varying the separation of sutures on the vessel ends, thereby causing "skewing", which might help to anastomose vessels of differing diameters (Fig. 3.19).

8. The combination of fewer jump-outs or RV "slip-back" leaks, with an automatic, atraumatic and reliable method of invagination, should lead to consistently faster anastomoses. 


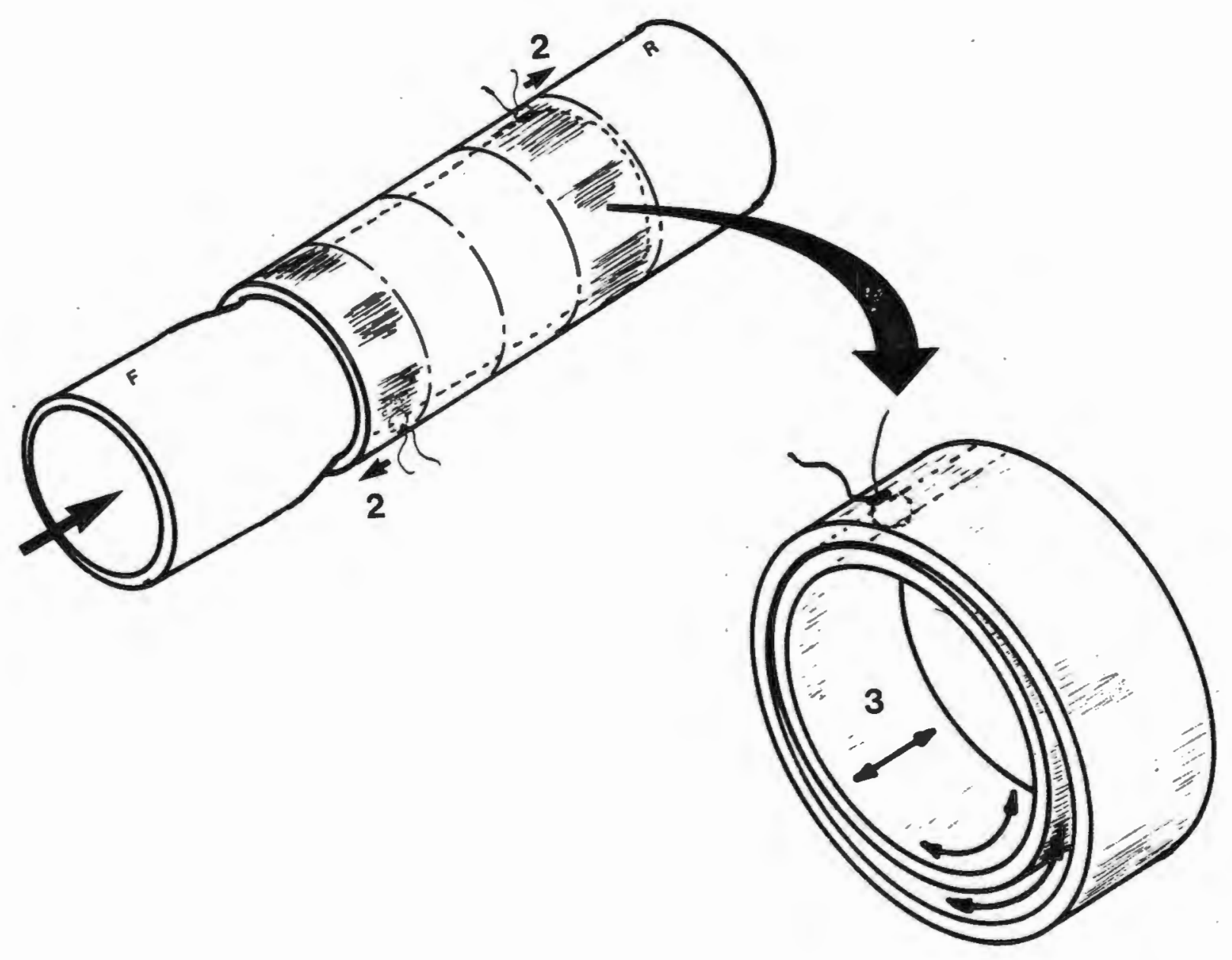

FIGURE 3.18

Only one suture on any single transverse section of the vessel. 


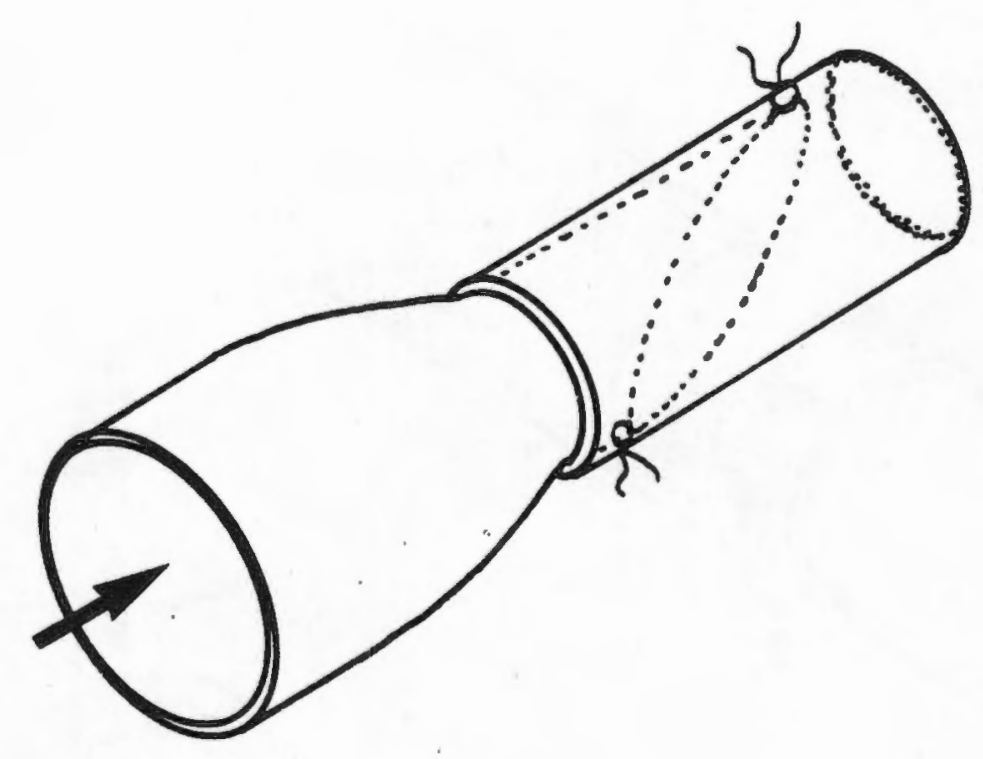

FIGURE 3.19

"Skewing" to anastomose vessels of differing diameters.

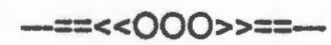

\section{NOTES}

1. Demonstrated to me by Prof. Harry J. Buncke (Microsurgery Course (1984): Microsurgery Laboratory, Ralph K. Davies Medical Centre, San Francisco.) 


\title{
CHAPTER IV. \\ SURGICAL TECHNIQUES FOR THE NEW ANASTOMOSIS
}

\author{
However much thou art read in theory, if thou hast no \\ practice thou art ignorant.
}

Sa'Di (1258)

\section{- INTRODUCTION}

The theoretical basis for the new EIEA had now been fully developed and required exposure to the realities of practice in the microsurgery laboratory.

In this chapter I intend to provide practical guidelines for the execution of the new anastomosis in a number of experimental and clinical situations. The "methods" sections of later chapters will be based on descriptions provided here.

\section{ANASTOMOSIS OF STANDARD MICRO-ARTERIES}

Vessels of approximately $1 \mathrm{~mm}$ in diameter have become the standard for microsurgery. The femoral arteries of rats $(108,109$,$) and rabbits (178)$ and the ear-vessels of rabbits $(24,207,208,206)$ have been widely used to evaluate microvascular anastomotic methods. The size of these vessels corresponds to that of the vessels typically requiring anastomosis in human replantation or free-flap surgery. 


\section{PREPARATION OF THE VESSELS}

Adequate and accurate preparation of the vessels forms the cornerstone of every successful microvascular anastomosis. A cursory preparation is a common mistake among novice microsurgeons, who are so eager to get on with the "real surgery" that they rush the preparation. They may then perform their suturing with meticulous care, only to see the vessel occluded by thrombus a few minutes after clamp release.

The first step in vessel preparation is the finding and identifying of the vessel. This is usually easy in the experimental laboratory, slightly more difficult in planned free-flap surgery and often extremely difficult in replantation surgery.

\section{DISSECTION}

Identified vessels are dissected free from their surrounding sheaths and accompanying vessels, in order to mobilise them sufficiently for application of the clamps and later approximation. Dissection is greatly facilitated by employment of the polished-needle cannula in its hydrodissection mode (see Appendix A). It may be necessary to divide minor branches of the artery to achieve adequate mobilisation and this should be done under control of micro-bipolar cautery or microligatures. Unipolar cautery should never be used anywhere in the vicinity of a microvascular anastomosis (30).

I have also found it most important when separating an artery from a vein or other delicate structure by blunt dissection, to ensure that tearing of interposed connecting tissues is done by grasping the unimportant tissue on either side of the proposed tear before applying the tearing force. This ensures that the required force is not transmitted via a vital structure which might itself be damaged by the force (Fig. 4.1). 


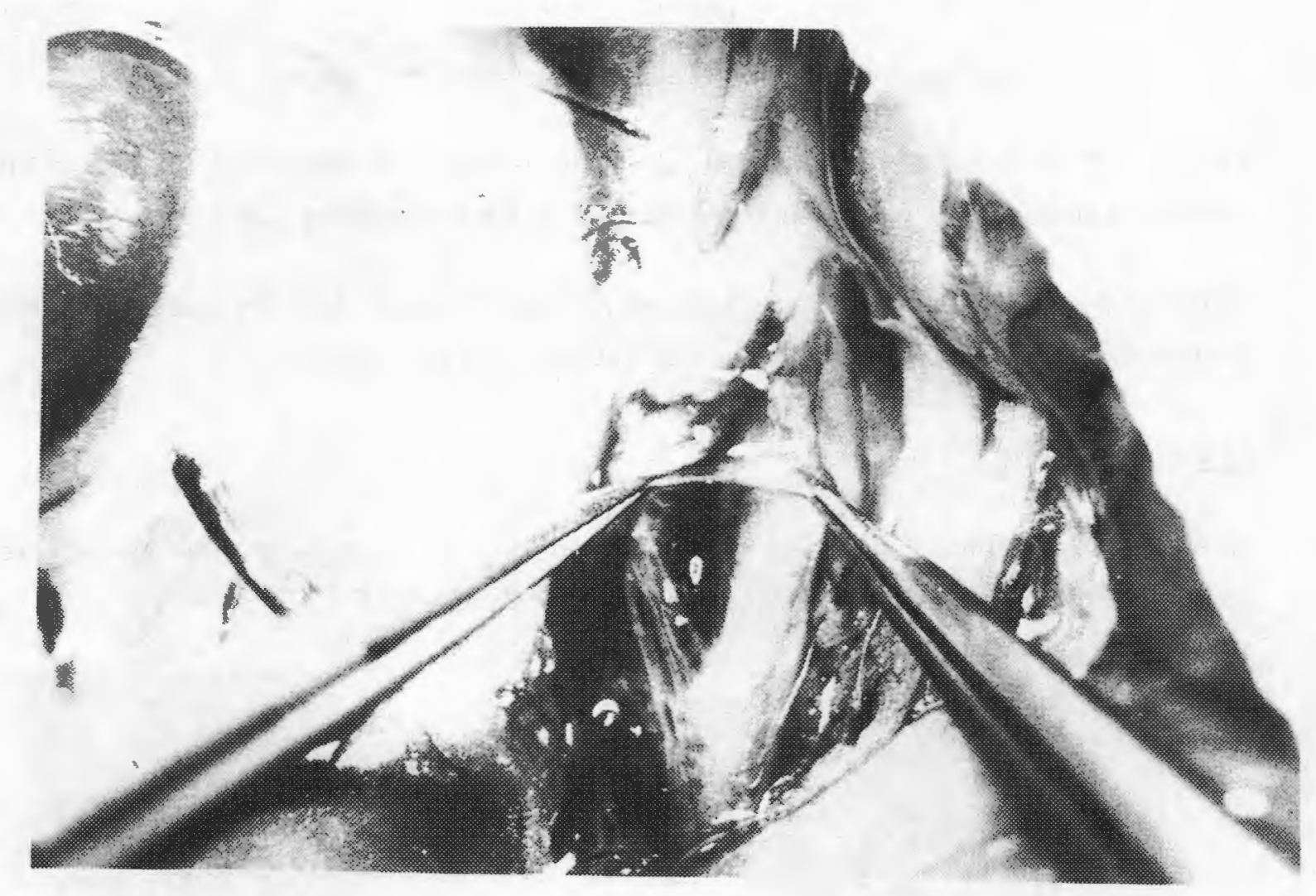

FIGUAE 4.1

Grasping the unimportant tissue on either side of the proposed tear before applying the tearing force.

\section{BACKGROUND MATERIAL}

Background material isolates the vessel from surrounding structures and improves the visibility of its structure. Small pieces of a toy balloon may be used in the laboratory. Sterile polyethylene material such as "Visibility Background Material" (Ethicon Inc. 1979, Somerville, New Jersey) is commercially available for clinical use.

I usually cut the background material to a width which is equal to the length of the bar of the approximating clamp that $I$ intend to use for holding the vessel. This width ensures that:

1. the clamp blades are able to lie on the material without slipping over the edges of the material on either of the two sides (this often happens when the material is too narrow); and 
2. the material does not protrude widely from under the sides of the clamp and thus snag the unclamped parts of the vessel with its edges.

I also cut off the corners of the material at $45^{\circ}$ to decrease the possibility of trauma to the vessel by a corner during placement of the background material (see Fig. 4.2).

Using two pairs of fine forceps, the background material is slipped under the vessel, which has been dissected free from its accompanying and surrounding structures.

\section{CLAMPS}

When the vessel stumps are adequately mobilised, they are carefully grasped in a double approximating clamp such as the Acland microvascular clamp (Fig. 4.2).

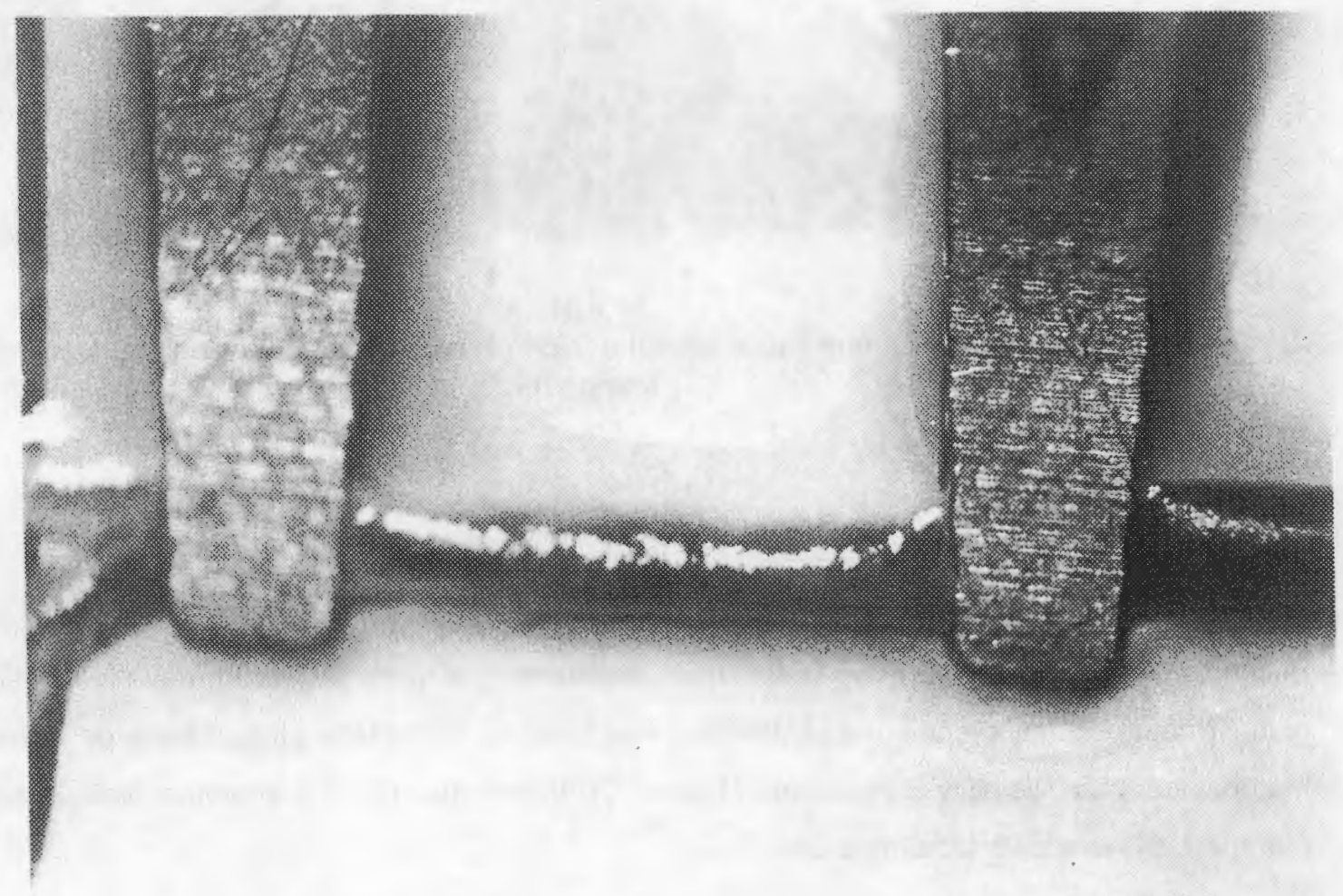

FIGURE 4.2

The vessel stumps are carefully grasped in a double approximating-clamp; such as the Acland microvascular clamp. 
Thurston et al (188) have demonstrated the damage caused to normal, healthy microvessels by vascular clamps. Such damage is likely to be more pronounced in vessels which have suffered the insult of being divided. It may be further compounded by incorrect choice of clamps or poor technique in their application. It is therefore necessary to select a clamp of an appropriate size and closing pressure for the artery in question. When using any of the specially designed sets of microvascular clamps which are now commercially available, $I$ find it a useful guide to choose the clamp which has jaw-blades of a width similar to the diameter of the vessel. The closing pressure, denoted by an " $\mathrm{A}$ " for arterial or a " $\mathrm{V}$ " for venous, is usually indicated on the clamp. I also consider it to be of vital importance to check, before applying the clamps, that they move freely on the bar and that the approximating mechanism is functioning adequately.

The chosen and checked clamps should be applied in the correct position at the first attempt. Despite the findings of Shaffer et al (171), I believe that removal and re-application of the clamps will lead to a greater potential for damage to endothelial and medial wall-layers. The correct position for the clamps is illustrated in Fig. 4.3. Note the fact that the clamped-off sections of vessel stump are at least 2 vessel diameters in length. 


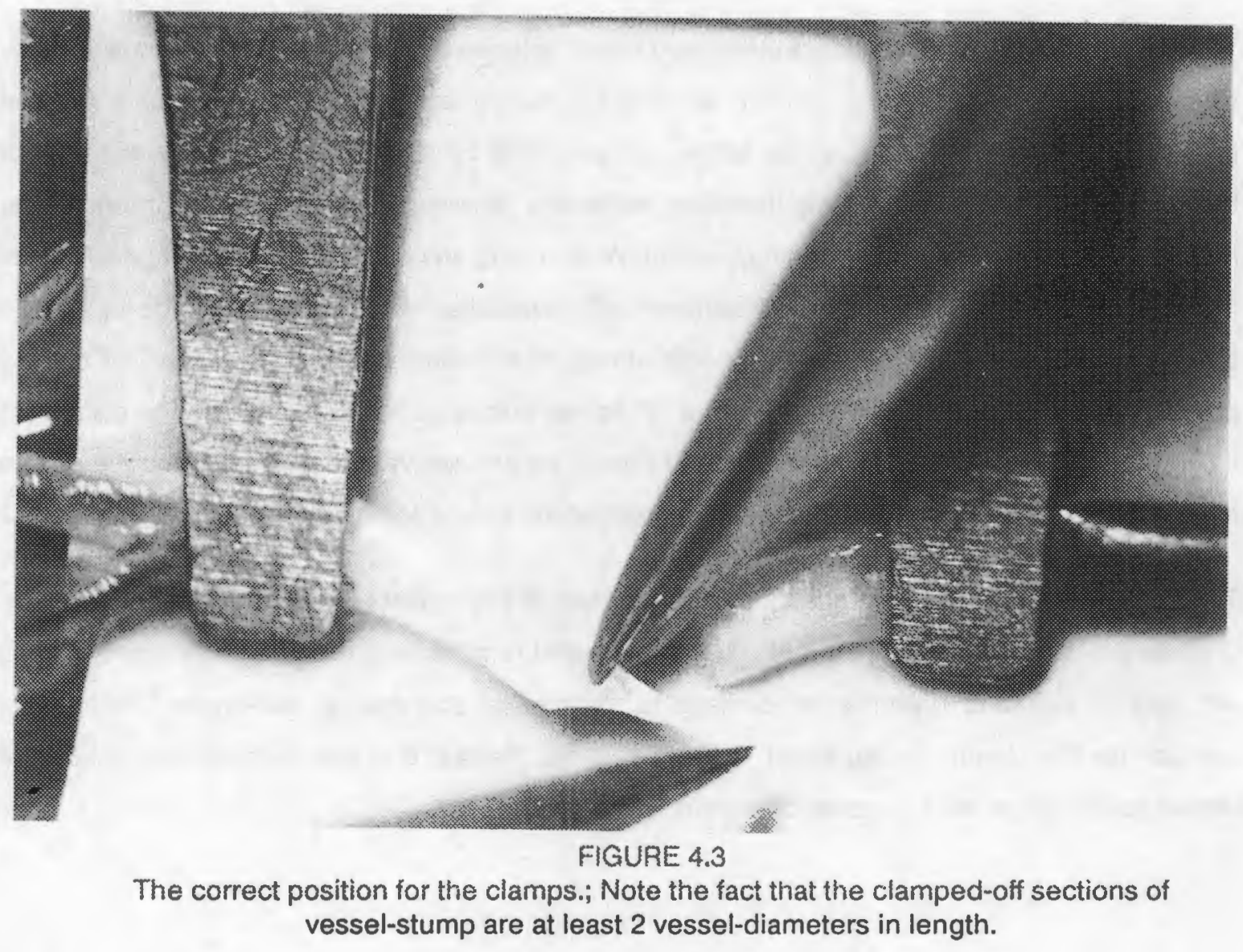

Finally, it should be remembered that the clamp is a tourniquet to the vessel stump. For the entire period of clamping, the vascular tissues in the peri-anastomotic area will be subjected to the effects of anoxia. It follows that prolonged clamping and consequent necrosis of vascular tissues in the region of the anastomosis will adversely affect the results.

\section{IRRIGATION}

Hiebert and Jaques (82) showed that heparin binds selectively to the vascular endothelium and Essien and co-workers (60) showed that heparin pre-treatment inhibited the adherence of platelets to rabbit aortic endothelium, which had been in contact with thrombin. They also demonstrated the fact that the prevention of thrombin-to-endothelium contact resulted in low rates of platelet adherence. Their findings were essentially similar in vessels with damaged endothelium, as opposed to those with normal endothelium. 
Sinclair (173) found that, when vessels were irrigated with heparinised N.saline, $75 \%$ of his experimental free-flaps survived, while there were no survivors among flaps of which the vessels had been irrigated with N.saline only. He recommends that heparin should be applied immediately to "virgin" endothelium in elective anastomoses and that application should be continued throughout the operation, thus "fastidiously" preventing all blood from entering the open vessel lumen (to prevent thrombin-to-endothelium contact). He also recommends a higher than usual concentration (50i.u./ml) of heparin in the irrigant.

I use an irrigating solution consisting of a fresh mixture of Ringer's lactate with heparin 30i.u./ml and Lignocaine $\mathrm{HCl} 1 \mathrm{mg} / \mathrm{ml}$. The lignocaine helps to overcome vasospasm and may also provide analgesia at the operation site in the early postoperative period. I prefer Ringer's lactate or a balanced solution of electrolytes (Plasmalyte B or Balsol) to N.Saline as a base for the irrigant, because they are less likely to cause electrolyte disturbances in the exposed endothelial cells during the clamping period.

The operating field is frequently and thoroughly irrigated with this solution, which is delivered from a syringe fitted with a polished-needle cannula. If the vessel is to be electively divided, all traces of blood are washed away from the operating field before division. Immediately after division all blood must be removed from the vessel lumen by a jet of irrigant.

Traumatically disrupted vessels must be trimmed and then irrigated to wash the lumen as clean as possible. The persistence of traces of coagulated blood in the lumen or on the endothelial surface may be indicative of covert damage to the vessel. It may then be safer to trim back further to an area where the endothelium has maintained its "non-stick" character and can therefore be cleaned with ease.

The copious use of irrigating fluid makes it necessary to have either a small suction device or a "wick drain" made of absorbent swabs in the operating field.

When the operating field has been cleared of all traces of blood, I like to leave a pool of irrigant around the vessel stumps to "float" the undesirable tissue elements and thus to make debridement easier.

\section{DEBRIDEMENT}

It is most important to perform an adequate debridement of the vessel stumps before performing any microvascular anastomosis $(185,78)$. Strands of adventitia exposed to the blood stream 
cause rapid thrombosis. On the other hand, the adventitia carries the vasa vasorum and Eisenhardt et al (59) have shown that removal of too much adventitia causes fibrinoid infiltration of the vessel wall, necrosis of the media and the development of aneurysms. Their work has been substantiated by Chignier et al (40). A sensible "conservative radicalism" towards adventitial strands must therefore be adopted.

I noted in my experience with the older EIEA methods that it was not necessary to trim the adventitia of the RV. This is true provided, that there are no long loose strands of adventitia attached to the cut edge of the RV. Such loose strands must be trimmed off. The remaining RV adventitia is then moved back from the edge by stroking it away with a smooth instrument.

The FV adventitia must be trimmed for two reasons:

1. The cut edge of the FV (and any associated adventitia) will be exposed to the blood stream at completion of the anastomosis.

2. Removal of adventitia "de-bulks" the vessel wall, allowing for a better telescoped fit with less cause for stenosis of the vessel's lumen.

The method for removal of the adventitia is shown in Fig. 4.4. It is important to pull the adventitia forward over the end of the vessel and then to cut by using tiny bites of the scissors, distal to the edge of the medial and intimal layers, which can be discerned through the adventitia. The cuff of intima is thus removed in toto, but not with one wild snip of the scissors.

The walls of traumatically divided vessels should be scrutinised to detect areas of intramural extravasation, distortion, or "forward longitudinal pulsation" (1), which may all be indicators of intimal damage and attendant vascular occlusion. 


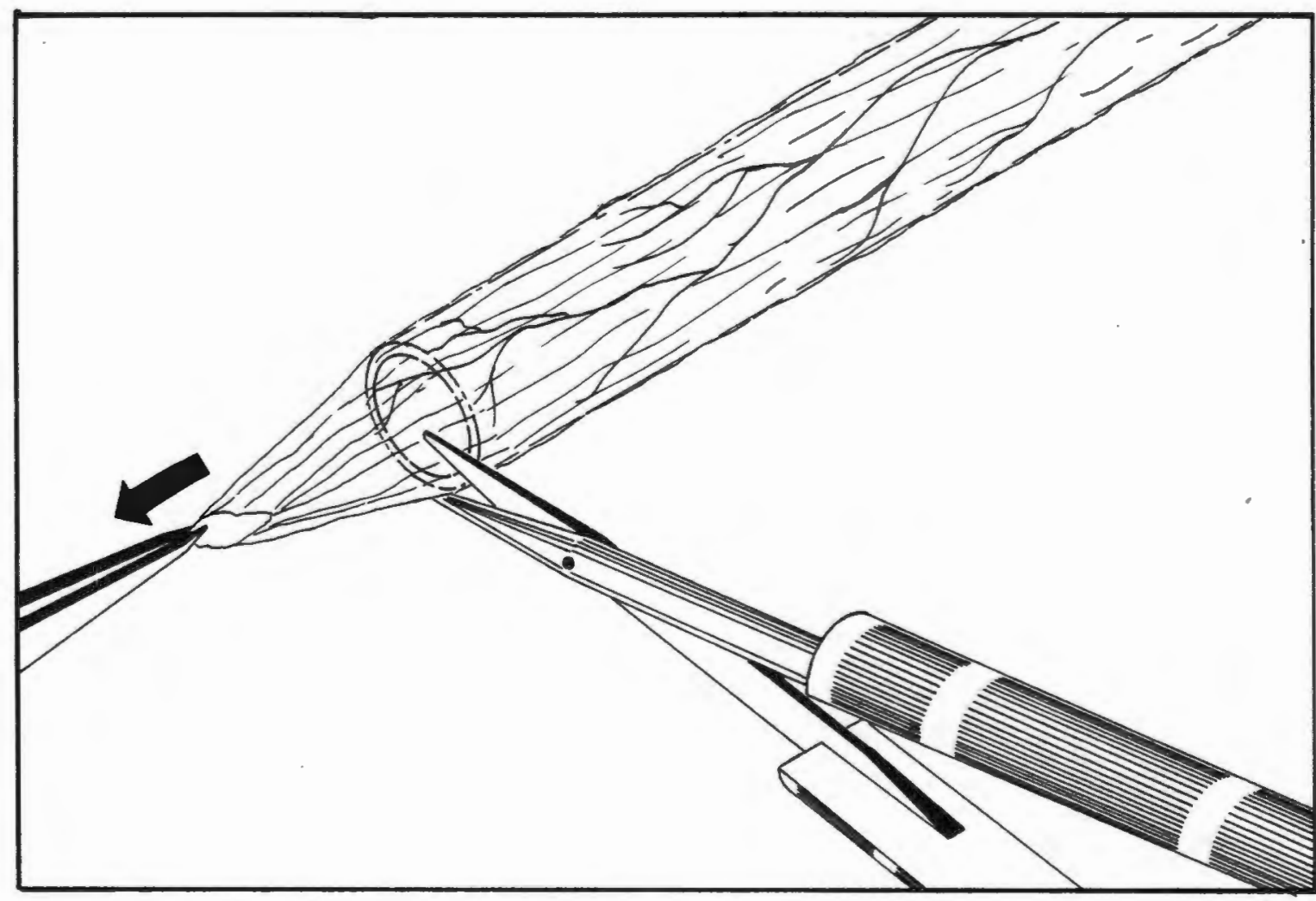

FIGURE 4.4

The method for removal of the adventitia.

Damaged areas must be resected and defects must then be bridged by means of autologous vein grafts. It was O'Brien who said (147,[p.55]):

"Repair of normal vessels: This is the most important principle in all microvascular surgery. Failure to work with normal vessels leads inevitably to impaired flow and a high risk of thrombosis"

Most microvascular surgeons will agree that the success of microvascular anastomosis depends more than anything else upon the joining of normal, healthy vessels.

It is often thought to be necessary to check that the flow from the debrided and cleaned vessel is unimpaired before proceeding with the suturing of the vessel. Bearing in mind that release and re-application of the clamps may not only cause clamp-related damage to the vessel wall, but will also release a lot of blood into the operating field, causing potential thrombin-endothelial 
contact, I venture to suggest that, with the availability of the quick and simple new EIEA, it may be prudent to rather perform a flow test after the anastomosis is completed. If there is an occlusion at another site in the vessel, the affected segment can be resected and the anastomosis repeated.

\section{DILATION}

Both vessels are dilated by means of the smoothly polished Acland's vessel stretching forceps (Fig. 4.5). Care should be taken to stabilise the vessel, so that it does not slip along the limbs of the stretching forceps when they are parted to apply their stretching force.

Stretching should be carried out with deliberate slowness to ensure stability of contact between the vessel wall and the stretching forceps. Before removing the forceps they should be closed halfway to decrease their wall-contact pressure, but not far enough to grip and damage the intimal surface during their removal.

Lauritzen pointed out that in the EIEA both vessels should be "maximally dilated" to "paralyze" their smooth muscle for some time and to decrease the ratio of the wall thickness to the lumen diameter (114).

My own experience has been that failure to dilate the inner vessel usually causes prolonged leaking, whereas adequate dilation of both vessels frequently leads to anastomoses which are completely dry from the outset. I attribute this to the fact that a dilated inner vessel will adapt itself more readily to the shape of the inner surface of the outer vessel and thus achieve better intermural coaptation in the entire overlap area. This is due to the fact that the dilated inner vessel is softer, thinner-walled and more pliable than an unstretched vessel.

I have reservations about the term "maximally dilated", since I believe that this could lead to over-zealous dilatory activity, which could seriously damage the intima and media of the vessel. This would lead to either scarring and cicatrisation or weakness and aneurysmal dilation with their attendant complications. 


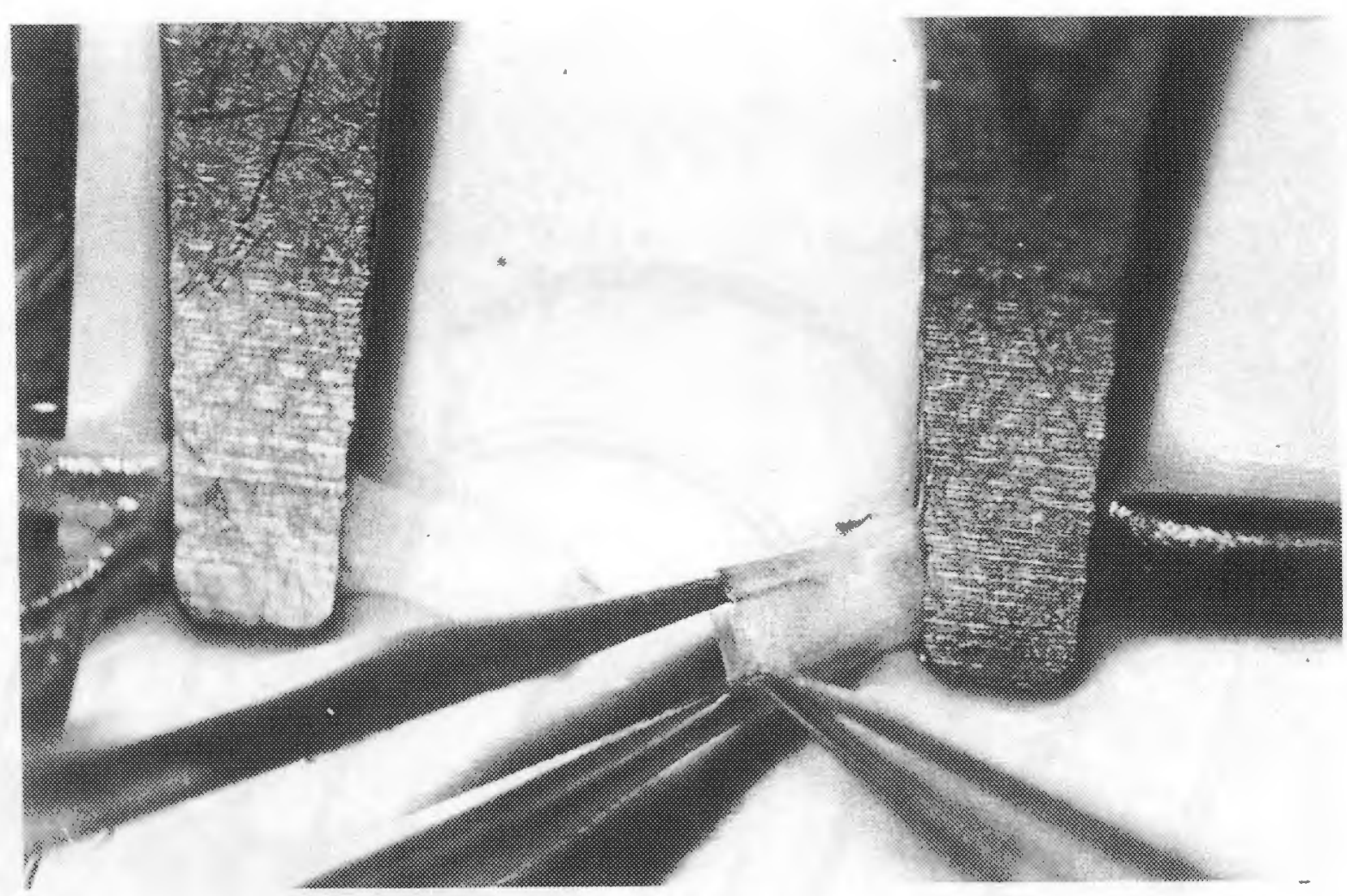

FIGURE 4.5

Acland's vessel stretching forceps.

\section{PLACING THE SUTURES}

A 10/0 monofilament nylon suture is usually appropriate for a $1 \mathrm{~mm}$ vessel. I use a 3/8-circle taper-point round-bodied needle with a shaft diameter of $75 \mathrm{~m}$ to $100 \mathrm{~m}(0.075 \mathrm{~mm}$ to $0.10 \mathrm{~mm})$ and a chord length of 3mm-4mm. The BV75-4 [ETHICON, INC., 1979, Somerville, New Jersey, U.S.A.] and the Dm 211 [SSC Inc. Schaffhausen, Switzerland] shown in Fig. 4.6 are such needles. 


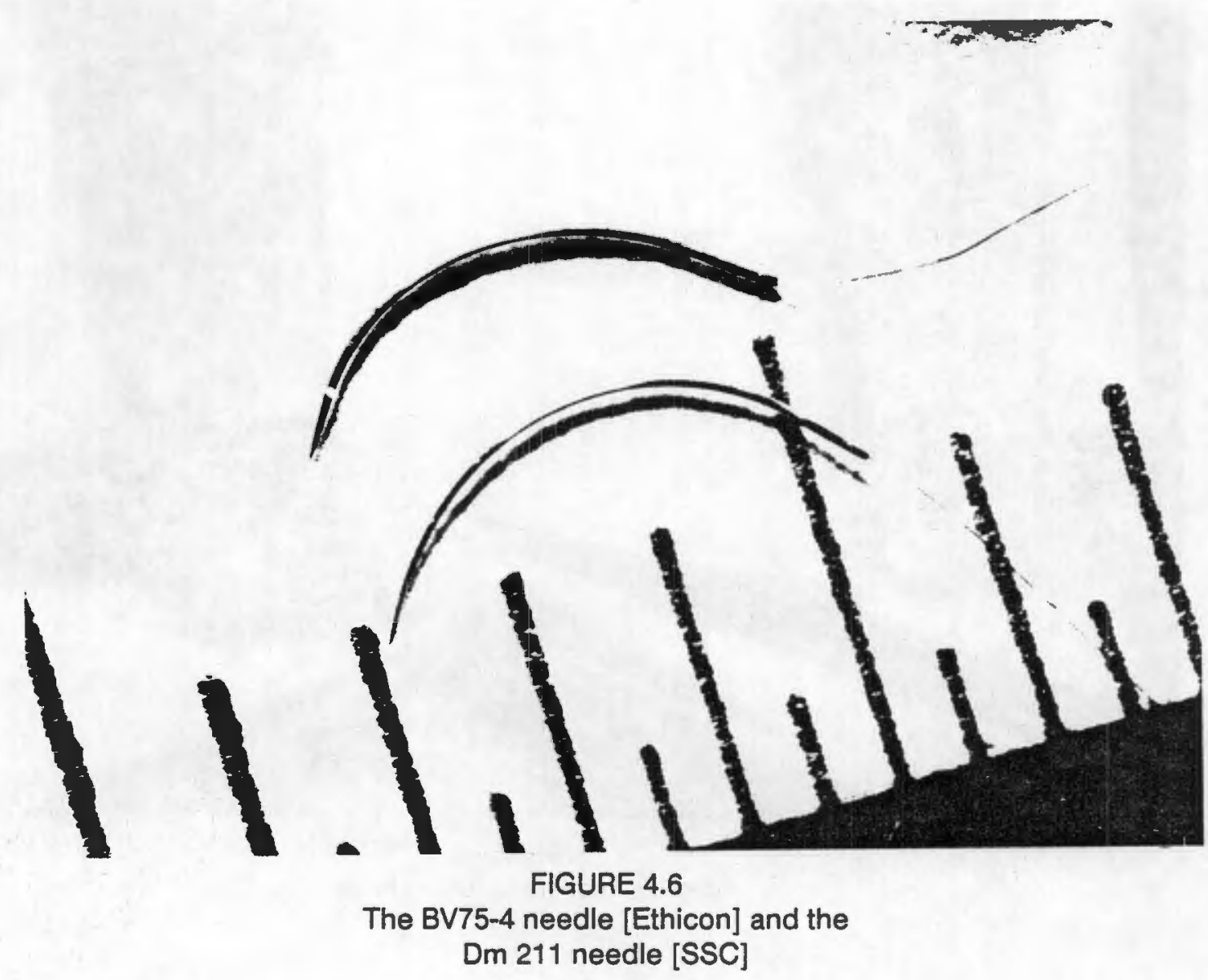

Before placing the sutures, I have often found it useful to take a "practice swing" over the top of the vessel to ensure that everything is positioned in such a way that the suture can be placed easily and correctly. The "practice swing" often reveals the presence of obstructions or snags outside the microscope field and these can then be removed before proceeding with the actual suture placement. 


\section{1st Suture Loop}

The deep looped suture is placed first. The polished-needle cannula is placed within the lumen of the vessel to a depth of 1.5 vessel diameters and used as a counterpressure device and backwall protector for the first needle pass (Fig. 4.7).

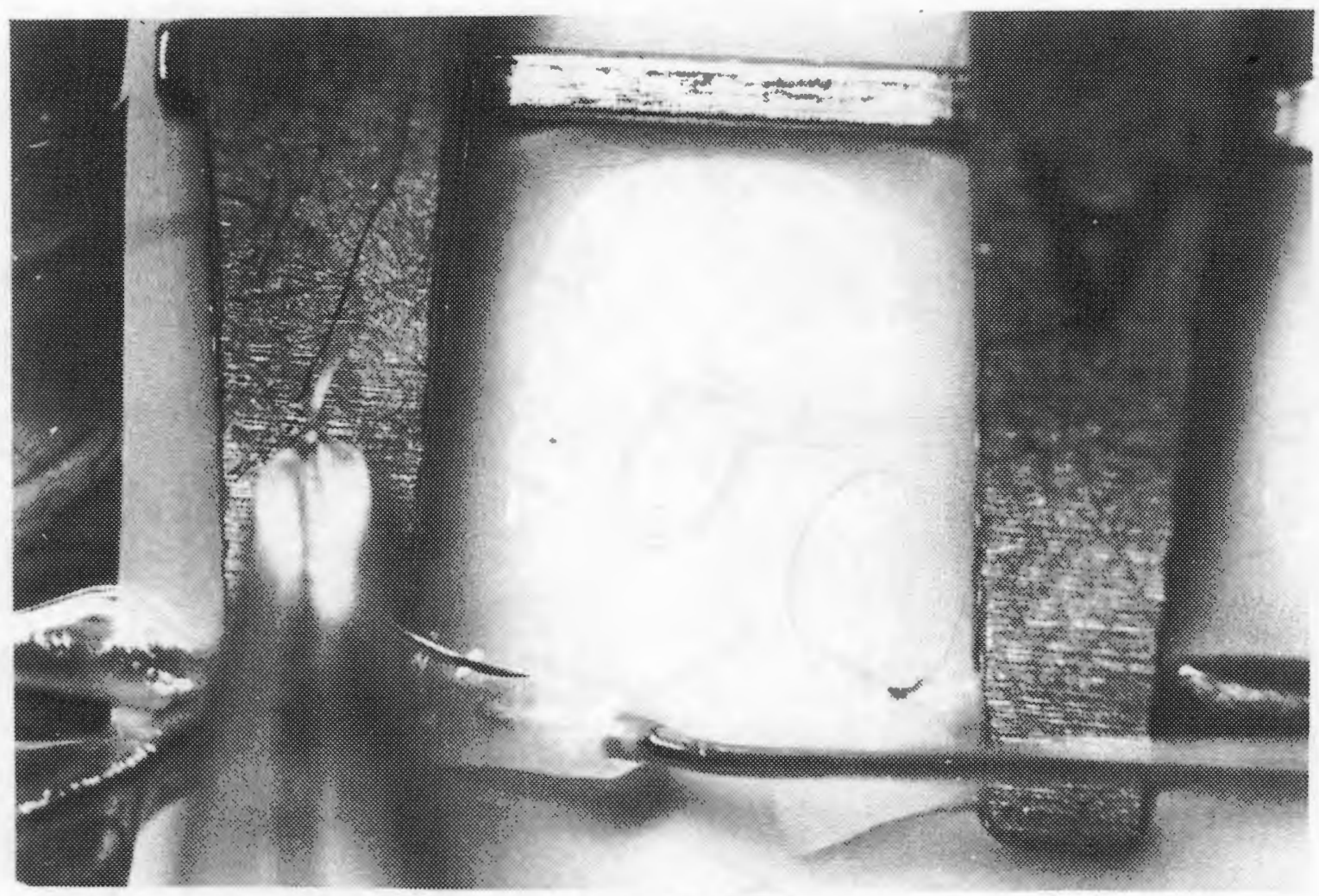

FIGURE 4.7

The deep looped-suture is placed first with the polished-needle cannula in attendance. 
The polished-needle cannula is held in such a way that its open end is vaguely visible through the opalescent vessel wall (Fig. 4.8[A]). The suture needle is passed through the vessel wall and into the lumen of the polished-needle cannula (Fig. 4.8[B]), which is then withdrawn while the suture needle is advanced in a totally guarded manner (Fig. $4.8[\mathrm{C}]$ ) until it emerges from the open end of the vessel.

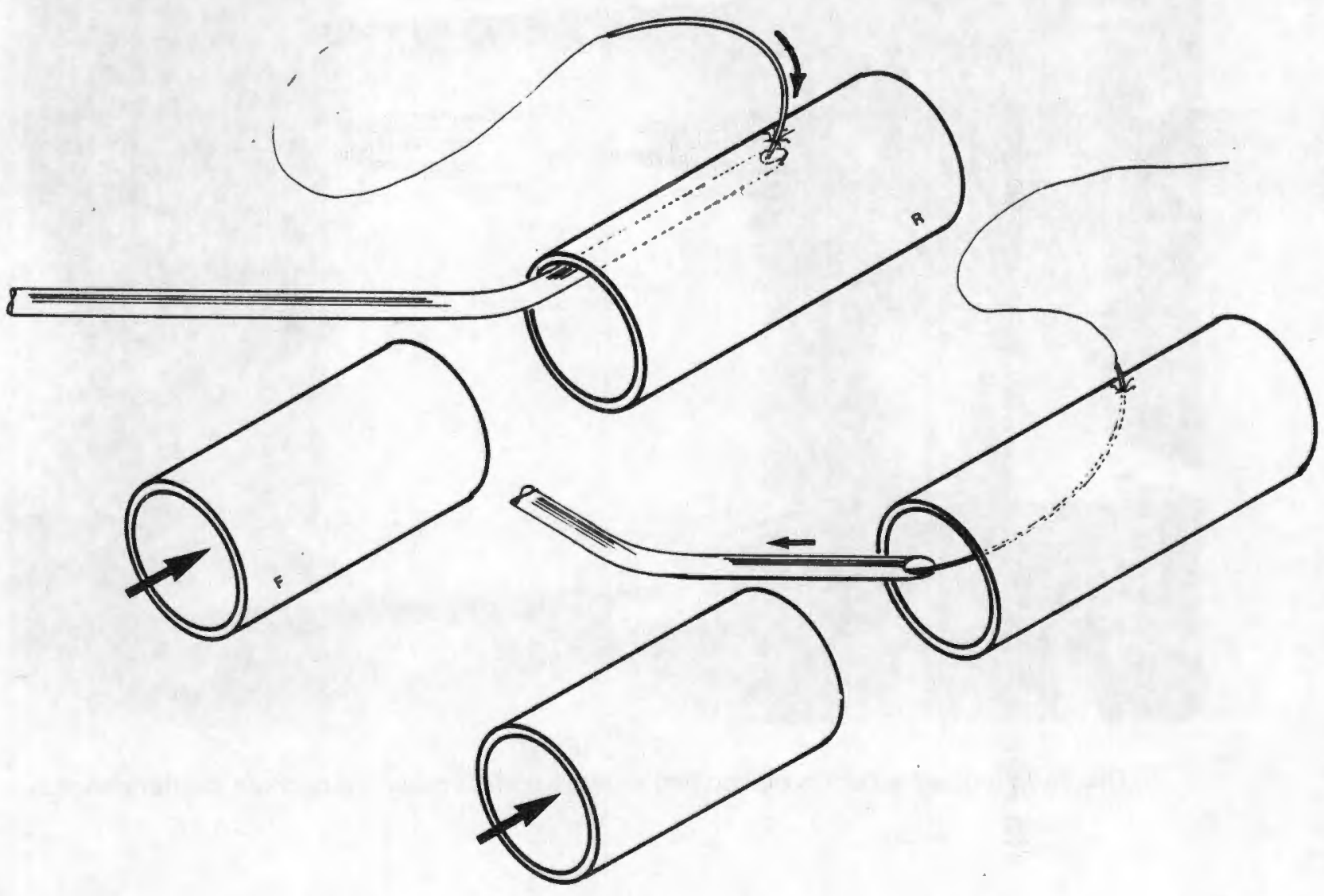

FIGURE 4.8

Suturing method for the new sleeve anastomosis 
The needle is next carried to the end of the FV, where a bite of only the outside layers of the vessel is taken by passing the needle tangentially through the vessel wall. This is done at a distance of approximately one wall-thickness behind the cut end of the vessel and in rotational alignment with the first needle pass in the RV (Fig. 4.9[A]).

The suture needle is then taken back to the RV and passed through the lumen to penetrate the vessel wall at a point adjacent to the entry of the first pass. This pass is most easily accomplished by taking hold of the suture material already lying in the lumen and lifting it so as to lift the front wall of the vessel away from the back wall (Fig. 4.9[B]). The suture material is guided through its course and broken off or cut off, thus leaving an untied, flat loop of suture material bridging the vessel ends. The two loose ends protrude from the wall of the RV (Fig. $4.9[\mathrm{C}])$.

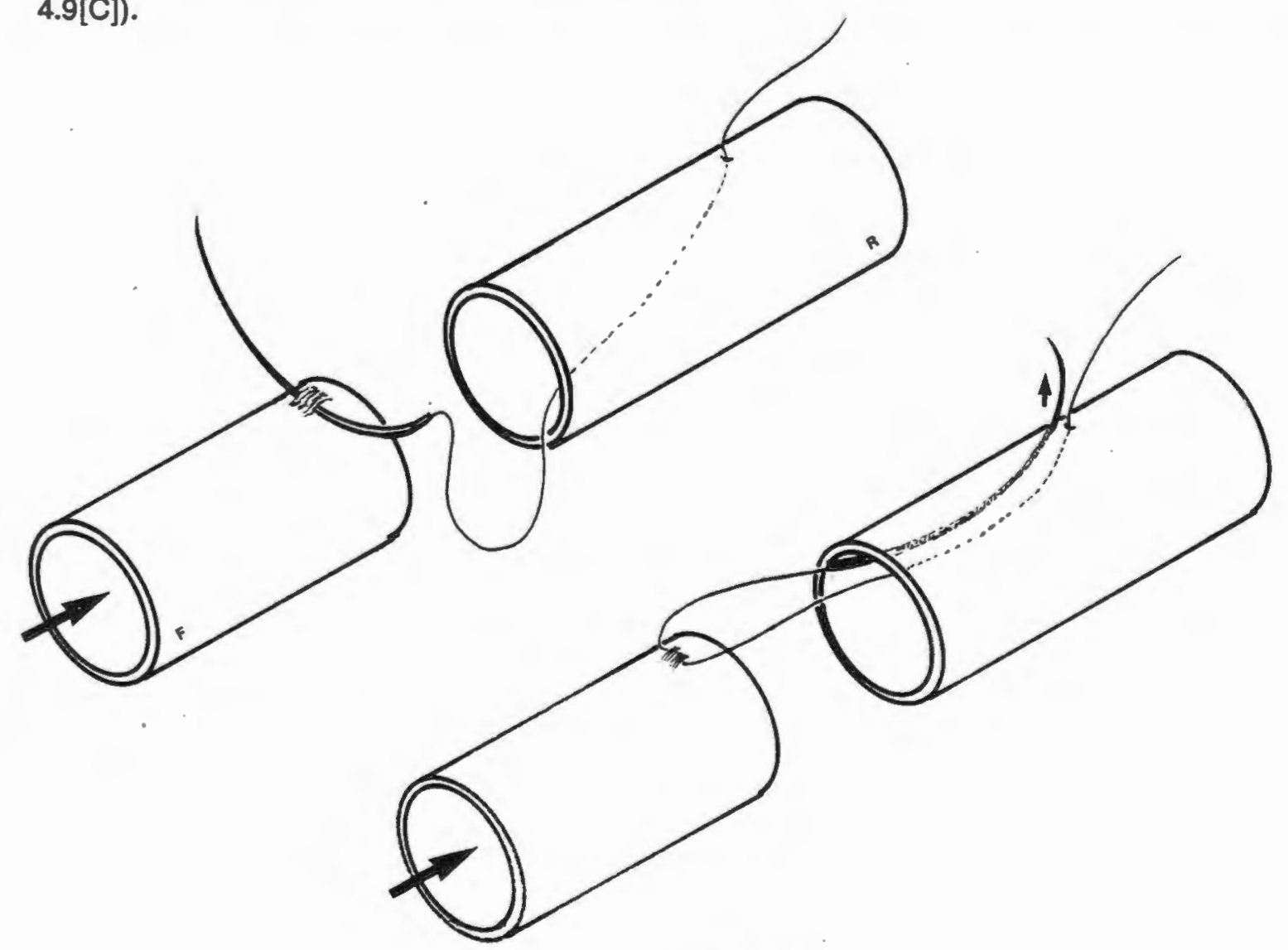

FIGURE 4.9

Suturing method for the new sleeve anastomosis 


\section{2nd Suture Loop}

The second suture loop begins in the same way as a standard vessel suture. It passes from outside to inside through the entire thickness of the wall of the RV at a point which is approximately one wall- thickness from the cut end and as close as possible to being diametrically opposite the first suture. The needle is then carried to the FV stump, where another superficial tangential bite is taken of the outer layers. This is done at a distance as far back from the end of the FV as the first entry point in the RV is from its end and keeping the circumferential distance between the loops in the two vessel ends equal. The suture needle is then taken back to the RV and passed from inside to outside through the wall next to the first pass of the second loop (Fig. 4.10[A]).

Both loops are then tugged neatly into place (Fig. 4.10[B]).

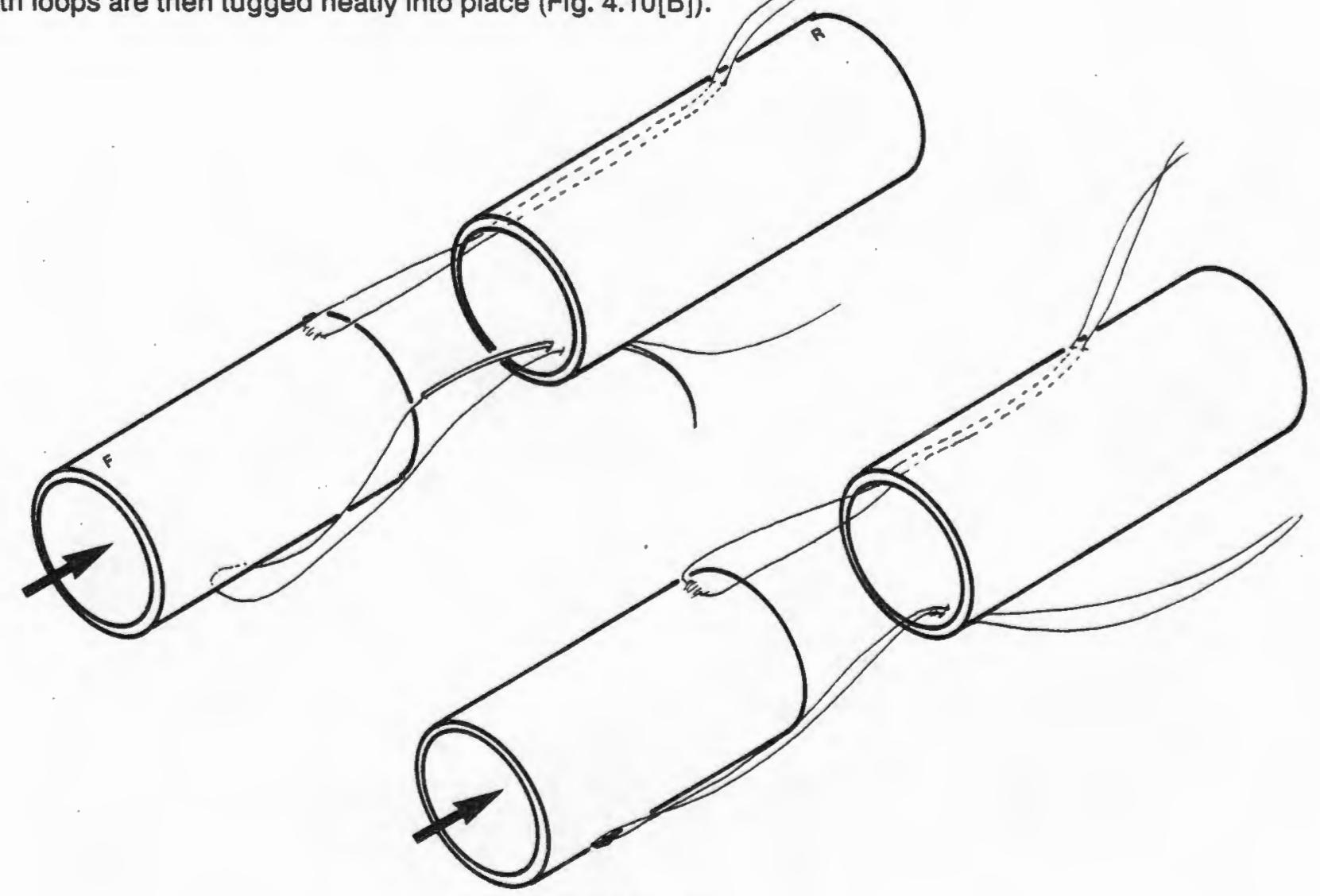

FIGURE 4.10

Suturing method for the new sleeve anastomosis 


\section{INVAGINATION}

Telescoping of the vessels cannot be adequately performed if one has to contend with the elastic tension in the vessels, which tends to oppose the telescoping procedure. It is therefore necessary to employ the approximating capabilities of the bar clamp.

\section{APPROXIMATION}

The background material is checked to ensure that its edges will not be hitched up under the vessels when they are approximated. If there is doubt, the material is either removed completely or trimmed.

The clamps are approximated at least the same distance as the overlap or more if vessel lengths permit. This will ensure that the FV lies in a completely relaxed manner within the RV when invagination has been completed.

\section{TELESCOPING}

The surgeon picks up each pair of suture ends with a forceps and gently pulls loop 1 in the direction of the blood flow and loop 2 in the opposite direction (Fig. 4.11[A]). This causes the vessel ends to slide automatically and without any further extraneous manipulation into the correct telescoped position (Fig. 4.11[B]). During this process the loops of suture material act as "guide rails" on either side of the FV so that it is both pulled and guided into position.

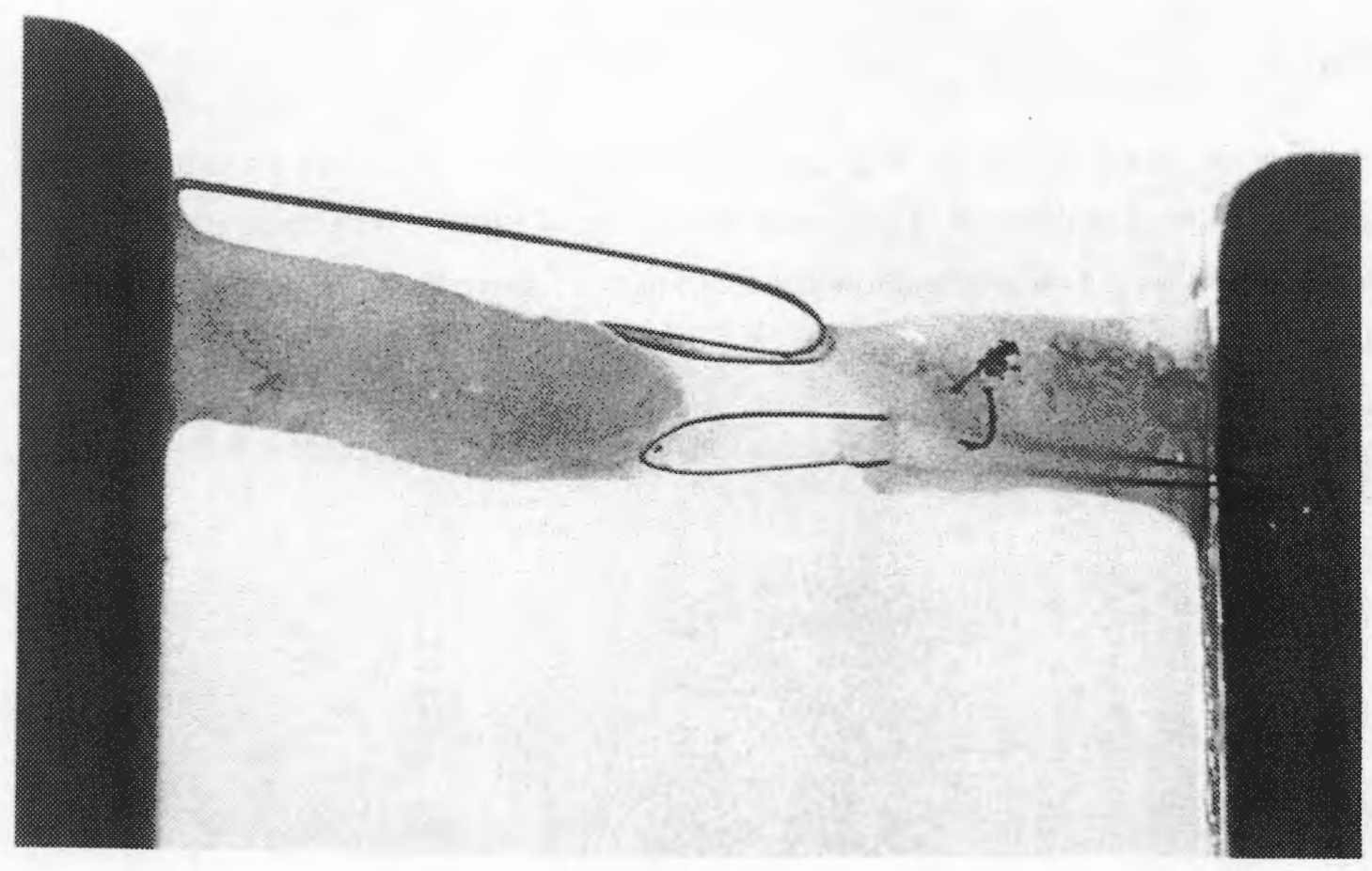




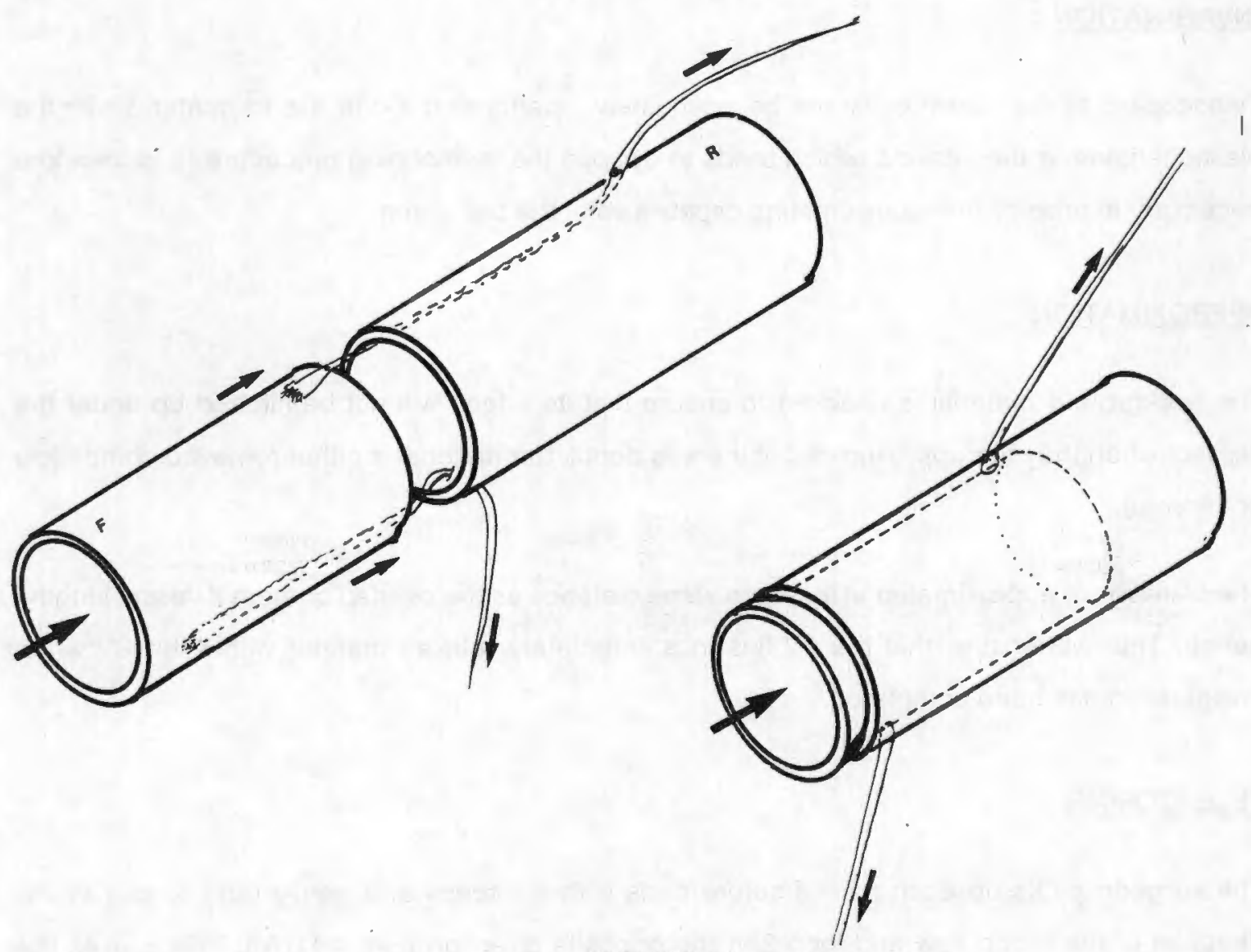

FIGURE 4.11

Suturing method for the new sleeve anastomosis

\section{FIXATION}

Loop 1 is tied first, taking care to lift while running the first throw home so that the knot is tied on top of and not in front of the end of the inner vessel (Fig. 4.12[A]). The second loop is tied down in the same way. I tie single-throw flat reef knots so as to keep the knots as small as possible.

All loose ends are cut short (Fig. 4.12[B]), especially those of loop 2, to assist with the next step in the procedure. 


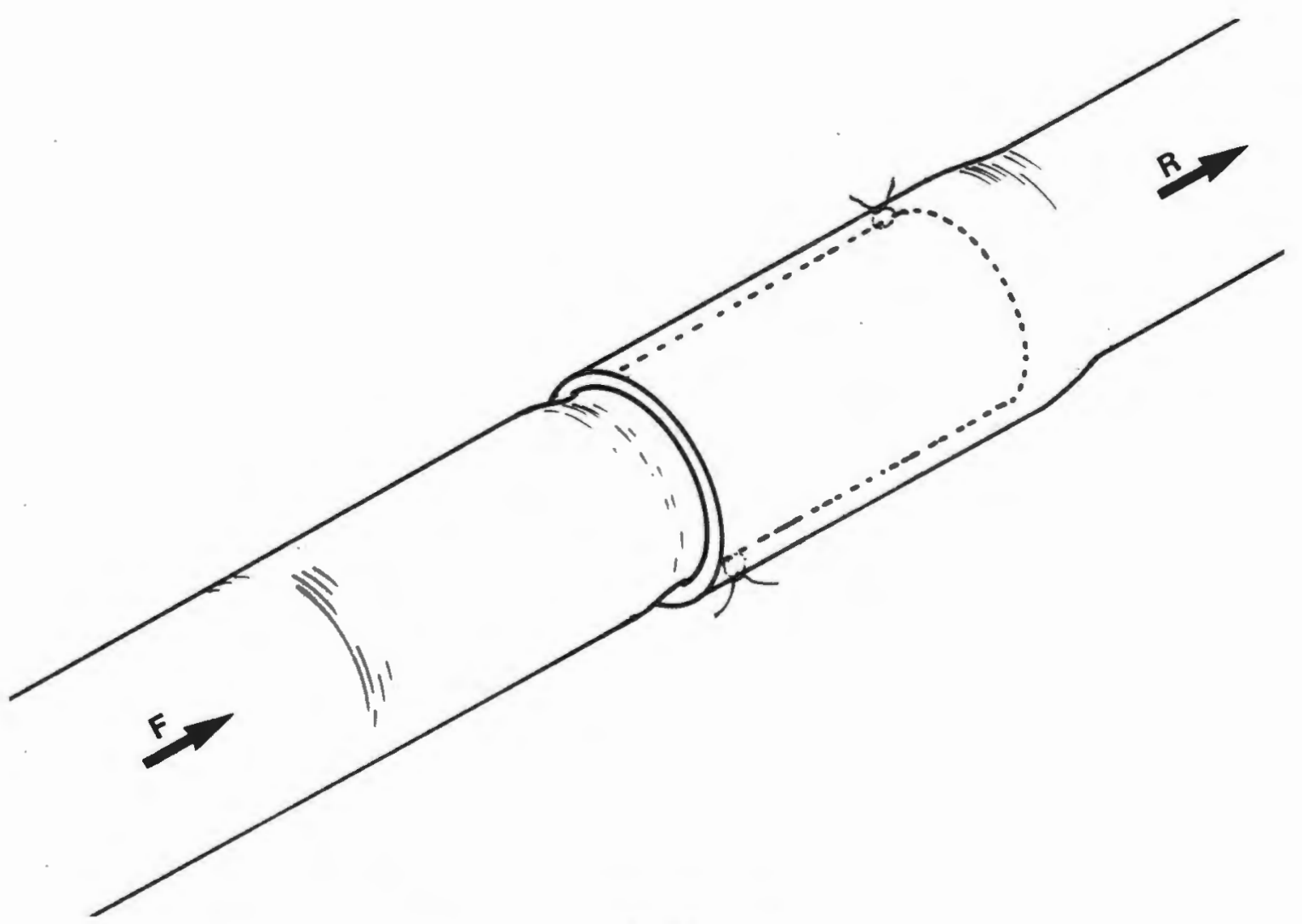

FIGURE 4.12

Suturing method for the new sleeve anastomosis

\section{ADVENTITIAL ADVANCEMENT}

The adventitia which has been withdrawn from the edge of the RV (see debridement:) is now advanced over the cut edge again. It is easier to do this if the sutures have been cut short. I believe that this re-positioning of the adventitia may assist in the healing of the anastomosis by returning the minute vasa vasorum to their natural position and bringing them into close proximity with the damaged vascular tissues, which require a new blood supply for their healing process.

The adventitia of the RV becomes adherent to that of the FV, making the anastomosis almost invisible (Fig. 4.13). 


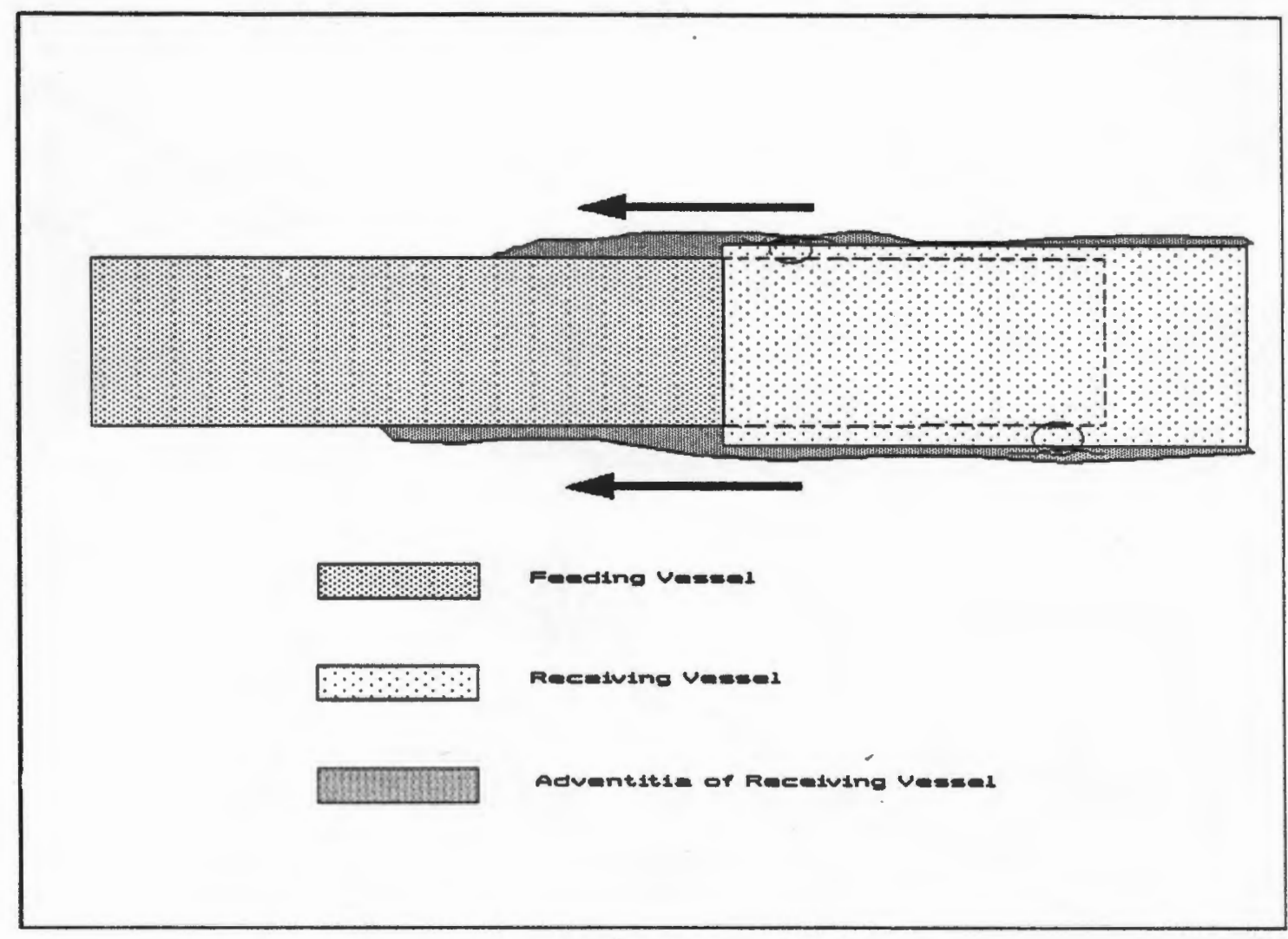

FIGURE 4.13

The RV adventitia is advanced over the cut end of the vessel.

\section{PREPARATION FOR CLAMP RELEASE}

I always find it expedient to spend a short time ensuring that the EIEA is ready for release of the clamps. When this preparation has been adequate, the clamps can be released with confidence. The following points are checked:

1. Look outside the clamps to ensure that the vessel is not unduly stretched.

If it is, dissect it free or relax the retractors which are holding the wound open. Failure to correct this situation will result in the elastic force in the vessel wall being transmitted directly to the anastomosis, which will in turn place stress on the sutures and the vessel wall immediately adjacent to the sutures. These 
areas of stress may cause puckering or folding of the vessel walls (Fig. 4.14). They may also prevent the movements that the walls of the two vessels have to make in relation to each other to find their optimal position of coaptation under the dynamic pulsatile conditions that the vessels will be experiencing after clamp release.

2. Ensure that the background material is not hitched up by the vessel, causing its edge to push against the vessel wall and resulting in partial occlusion of the vessel.

3. Clamp release in the case of large free- flaps or limb replantations may have important metabolic effects on the patient $(32,174)$. I therefore check with the anaesthetist at this stage to ensure that he is prepared for the consequences of my action. 


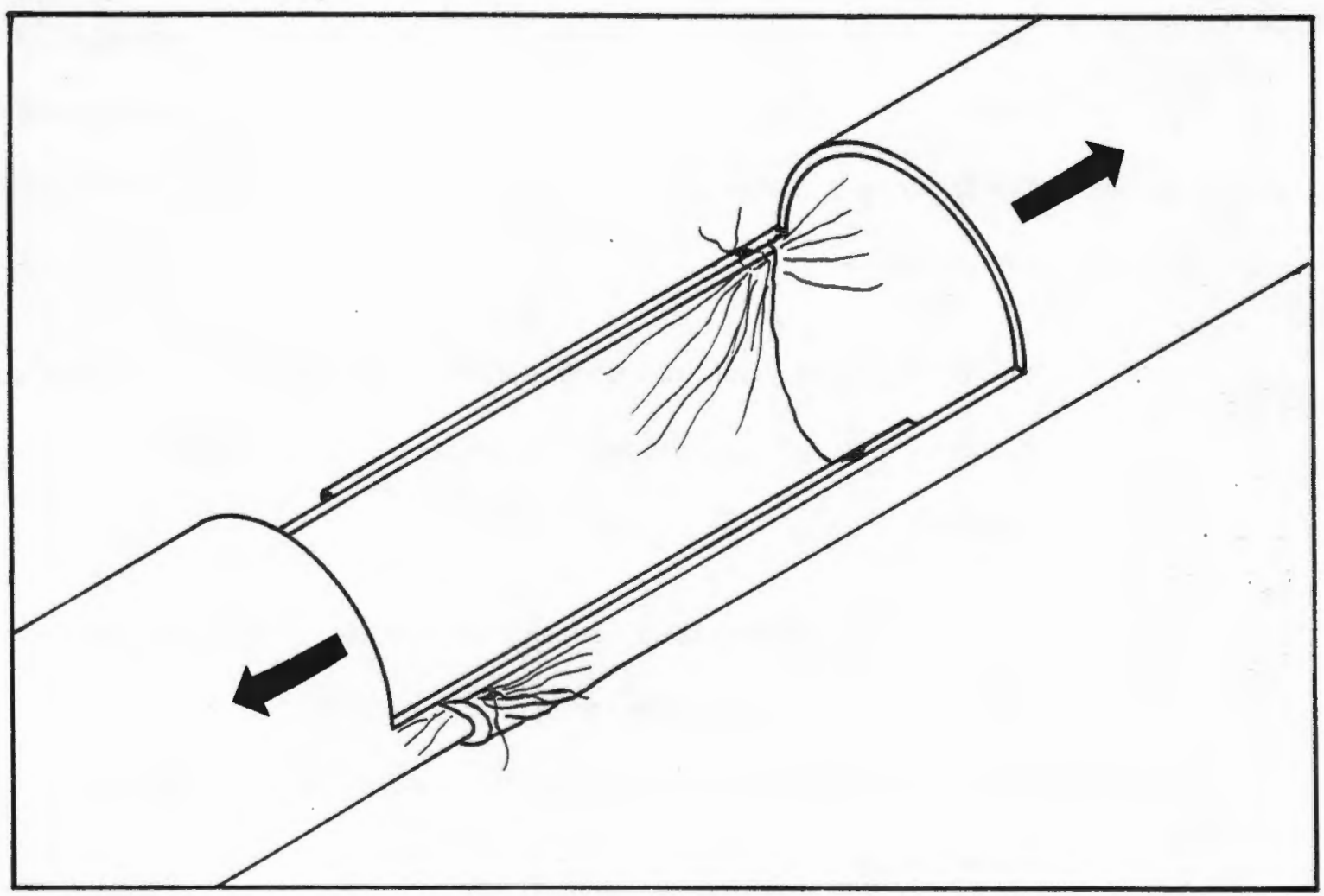

FIGURE 4.14

Areas of stress may cause puckering or folding of the vessel walls.

\section{FLASH RELEASE}

The clamp on the FV is momentarily and partially released. The position of the clamp is not changed at all. It is merely opened and almost immediately closed again, the whole procedure taking less than 0.25 seconds. The rush of blood blows the inner vessel out against the inner surface of the outer vessel's wall, thus pushing it evenly into place in a position which must be nearly the same as its final position after clamp release. 


\section{INTERMURAL CLEARING}

The end of the FV should now be clearly visible through the wall of the RV. The overlap area should appear lighter in colour than the rest of the vessel. This implies good intermural contact with no blood between the surfaces. If the overlap area is unclear or if a small amount of blood can be seen between the surfaces, the situation may be remedied by stroking the surface of the RV briskly in the upstream direction, i.e towards the end of the RV. This action will push the RV and FV walls together and squeeze the blood out from between them so that it emerges from under the edge of the RV. Any blood which may have emerged as a result of this procedure is washed away before the clamps are released.

\section{CLAMP RELEASE}

\section{SEQUENCE IN SINGLE VESSEL}

Having checked that the anastomosis is in order, the clamps are released in the following order:

first the clamp on the RV, followed immediately by that on the FV.

This order ensures that there is no backpressure when the high-pressure side is released and the speed with which the releases follow each other ensures that there is pressure to hold the walls together before normal backflow has an opportunity to dissect a path between the walls.

The anastomosis usually bleeds very little in comparison to standard ETEA techniques. This confirms the observations of Lauritzen (115).

\section{FREE-FLAP SEQUENCE}

In free- flap surgery, flow in the artery and vein are interdependent. If the vein is occluded, the tissue will become engorged to the point where pressure within the free-flap tissue is similar to the arterial pressure and no further inflow takes place. When this occurs, the stasis in the artery, where there has inevitably been some endothelial damage, might lead to thrombosis. Some surgeons complete the arterial anastomosis and remove the clamps to allow filling of the flap and free bleeding from the veins. They then re-apply the arterial clamps and complete the 
venous anastomosis. The rationale appears to be that this short period of perfusion will remove toxic wastes and also re-oxygenate the flap (27 $\mathrm{CH} 3 \mathrm{p} .17]$ ). I believe that the vascular system of the free flap or replant is in a safer condition when its anastomoses are kept completely clean until continuous blood flow can be re-established. If EIEA's are being used, it should be remembered that these anastomoses depend upon pressure and flow within them to maintain their integrity.

To me it seems best, therefore, to anastomose the veins first and to keep the clamps on the veins until the arterial anastomosis is complete and functioning. The venous clamp is released as soon as filling of the veins distal to the distal venous clamps is noted. The fact that the EIEA is much faster to perform than the ETEA supports the feasibility of this approach.

\section{IMMEDIATE ASSESSMENT OF ANASTOMOSIS}

\section{PATENCY}

Mechanical flow tests or filling tests (78) should not be performed within the first five minutes of clamp release and an even longer delay is preferable. The reason for this is that it is during this time that the inner vessel settles itself into position under the dynamic pulsatile pressure conditions and a seal is achieved between the vessel walls. Tests which alter the flow pattern or cause backpressure beyond the anastomosis can cause backward dissection, thus establishing an undesirable "leak channel" between the walls. There is evidence that the widely used "milking" test may cause severe damage to the vessel (152).

I prefer Acland's signs and tests (1) which, if carried out correctly, cannot harm the vessel. Of these signs I find the most reassuring ones to be the "wriggling" of an arterial branch distal to the anastomosis and the upstream distension of the vein after I have produced a downstream occlusion by means of fine forceps.

If the signs of patency are absent, the vessel could be physically completely occluded by intraluminal or extramural obstructions, or it may be affected by intramural factors. It may also be affected by intermural factors which are unique to the EIEA. 


\section{Intraluminal Occlusion}

Complete occlusion is as likely to occur in the EIEA as in any other type of anastomosis if one of the following mistakes has been made:

1. Damaged vessels have been anastomosed.

2. The adventitia has entered the lumen.

3. The backwall has been picked up in a suture.

4. Foreign organic material has been left in the Iumen.

5. Clotted blood has been left in the lumen.

The only way to remedy a complete occlusion is to take down the anastomosis and remove the cause of the problem. There is usually an associated need to re-trim the vessel stumps and sometimes an interposition graft is required to bridge the defect.

\section{Extramural Obstruction}

External causes of vessel occlusion should also be recognised and dealt with by the surgeon. These include snagging of the downstream vessel by other tissues or background material or, in the free-flap situation, occlusion of the venous return by clamps or ligatures. Snaggings should be' recognised and corrected before clamp release.

\section{Intramural Obstruction}

Intramural causes for obstruction to flow are:

(a) Downstream spasm.

(b) Intimal separation and dissection.

(c) Intramural haematoma. 


\section{Downstream spasm.}

This is usually treated by accurate application of small amounts of a $2 \%$ Xylocaine solution to the problem area, while avoiding application to the anastomosis itself. Geter et al (68) have shown chlorpromazine $25 \mathrm{mg} / \mathrm{ml}$ to be the most effective vasodilator for $1 \mathrm{~mm}$ blood vessels. They recommend Xylocaine $20 \%$ as a second choice. In this situation, however, it is necessary to use a mild vasodilator.

Application of vasodilators to the anastomosis in the early phase will potentiate the effects of the back-pressure by causing the outer vessel to relax before the inner vessel does so, thus causing separation of the walls with a resulting propensity for leakage.

\section{Intimal dissection}

This was the reason for Lauritzen's original search for an alternative suture method (115). The EIEA has been shown to be less prone to this form of occlusion (115), but it may still occur in isolated cases. When it does occur, the only practical course of action available is resection of the affected vessel segment.

\section{Haematoma}

An intramural haematoma indicates that there is a breach of the intima or that one of the vasa vasorum has ruptured. In either case the vessel must have suffered significant trauma in the region of the haematoma. The presence of such a haematoma should therefore be an indication for resection of the affected vessel segment.

\section{Intermural Obstruction}

When there is poor coaptation of the vessel walls in the overlap area, blood can collect between the walls, thus narrowing the lumen of the inner vessel or completely occluding it. This situation is analogous to the physical result of intimal dissection occurring in a different plane.

Unlike intimal dissection this situation is often remediable.

With the aim of expressing the intermural collection of blood and improving contact between the walls, a "Peanut" swab or a sterile cotton bud (pus swab) is rolled over the anastomosis in the 
opposite direction to that of the blood flow (Fig. 4.15). This squeezes the vessel walls together and expresses the blood from under the edge of the RV. The blood flowing into the lumen behind the "roller" then holds the vessel walls together, thus preventing a recurrence of the problem.

If this simple measure does not work, it usually means that there is a more fundamental problem to address. The root cause may be inadequate stretching of the inner vessel (see Dilation:). When this problem occurs in other EIEA procedures, it may be due to a disparity between the suture separation angles on the two vessels, but due to its "single suture per transverse section" characteristic, this is unlikely to be the cause in the new EIEA.

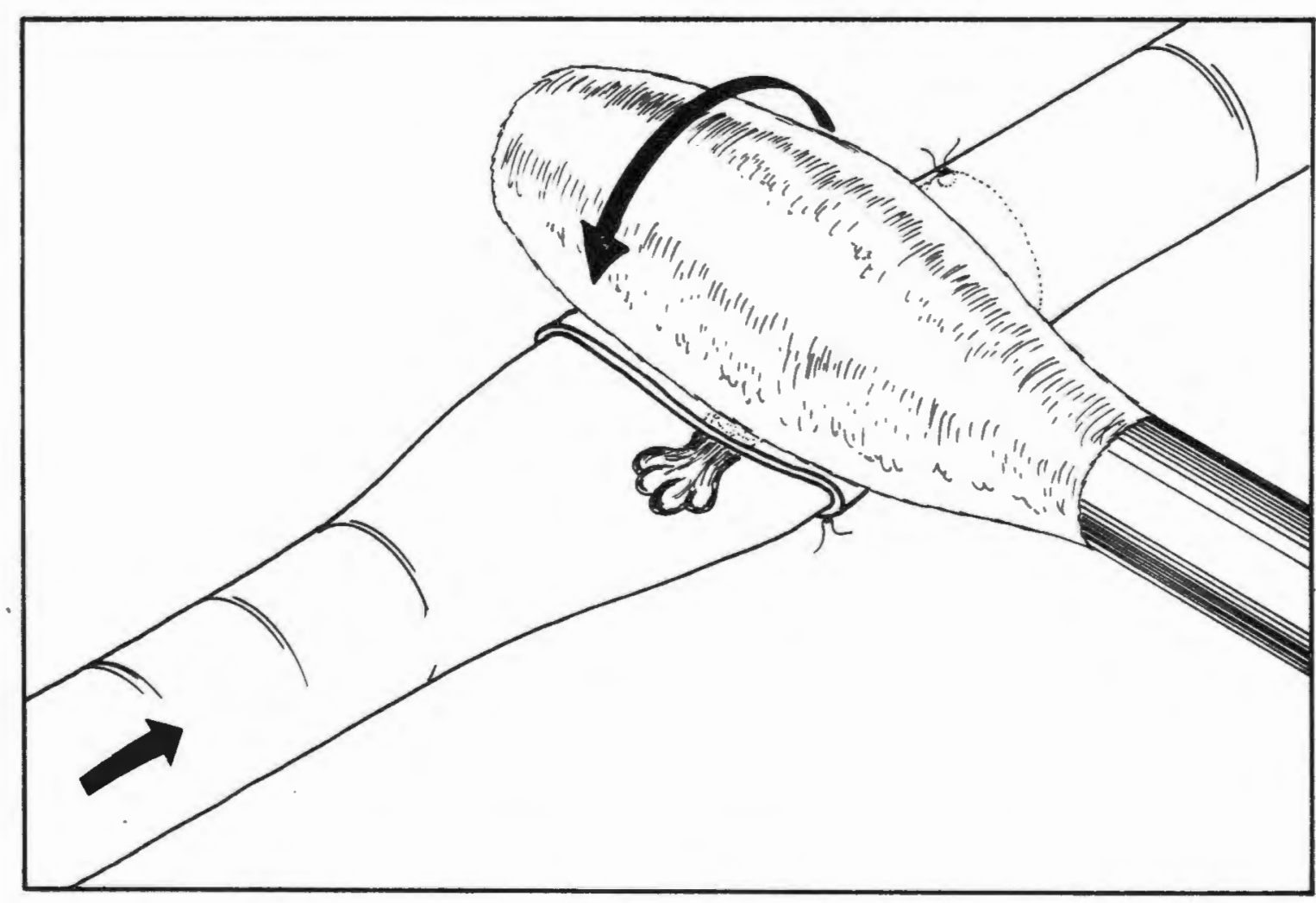

FIGURE 4.15

"Peanut" swab or a sterile cotton bud (pus swab) is rolled over the anastomosis in the opposite direction to that of the blood flow. 


\section{ADEQUACY}

A microvascular anastomosis which is patent, is not necessarily adequate. It may harbour inherent flaws which will result in an impairment of function, which may be temporary, permanent or progressive.

\section{Leaking}

In the new EIEA there should be no holes in any part of the vessel wall exposed to the blood stream. Any leaking must therefore take place between the walls of the inner and outer vessels. This implies that there must be an area of poor intermural contact which may enlarge, especially in the presence of downstream spasm or any other cause of backpressure which may bring about preferential flow through the leak channel. Poor intermural contact also means that there is no frictional force between the walls in the leak area thus decreasing the tensile strength of the anastomosis. As explained earlier, leaks can usually be stopped by the "roller" method.

\section{Stenosis}

Stenosis at the anastomotic site will impede blood flow and may alter local flow patterns, thus subjecting the adjacent vessel wall to abnormal stresses, which may in turn lead to thrombus formation and occlusion of the vessel (164). If the FV adventitia is correctly trimmed and both the $\mathrm{FV}$ and the RV are mechanically dilated before invagination, the repaired vessel is often dilated at the anastomotic site. The local sympathectomy occasioned by division of the vessel may add to the dilatory effect at the anastomotic site.

Late stenosis at the anastomotic site has been demonstrated by vascular corrosion casts (see Chap.6). The stenosis seen in these casts has always been uniformly smooth and streamlined (Fig. 4.16), clearly different to the irregular inner surface of a standard ETEA. This late stenosis may be due to fibrosis and cicatrisation of the vessel wall following necrosis of the media which, I believe, may be caused by over-zealous mechanical dilation of the vessels. 


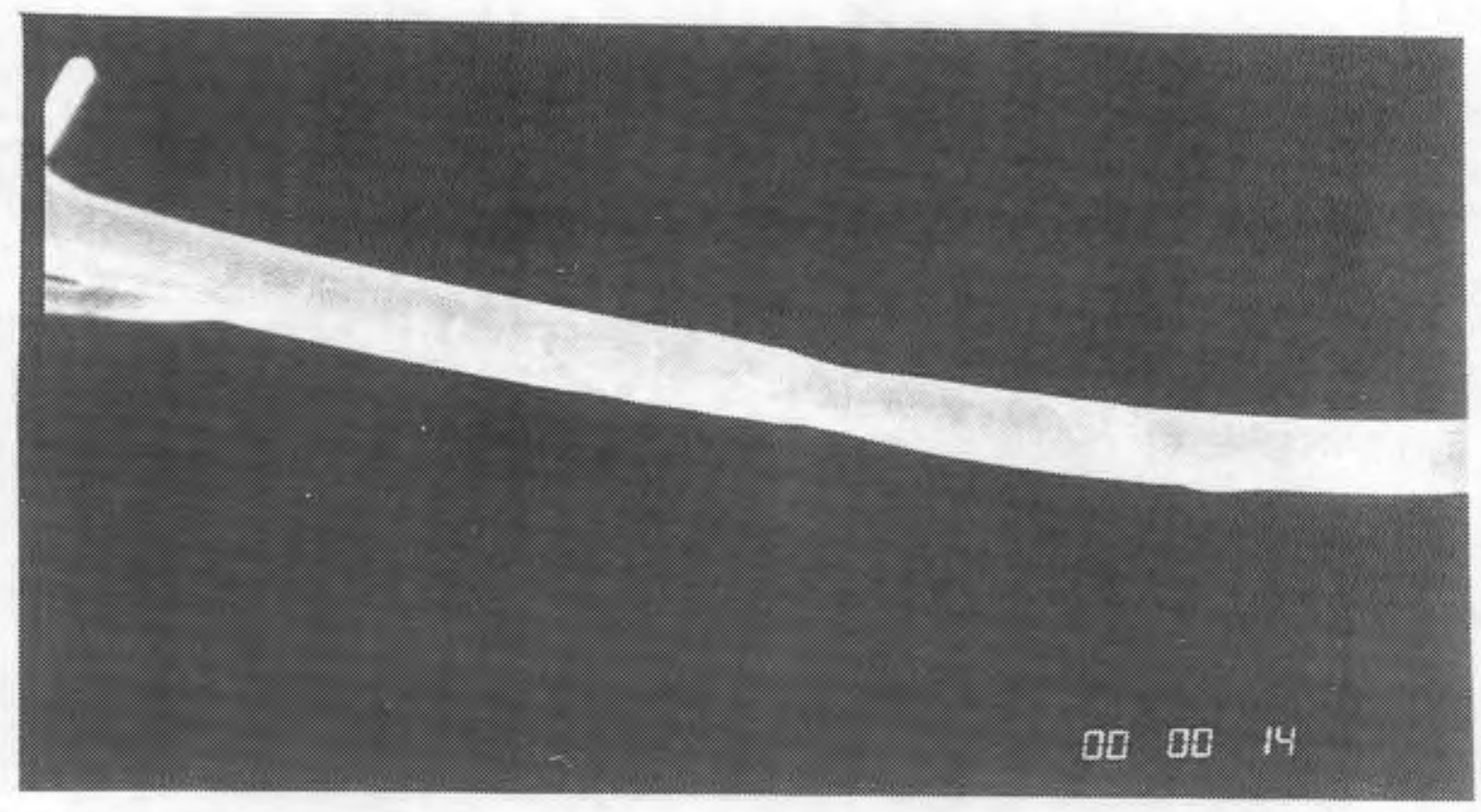

FIGURE 4.16

The stenosis seen in these casts has always been uniformly smooth and streamlined.

\section{Distortion}

Distortion of the vessel at the anastomotic site occurs frequently when novice microsurgeons are using interrupted-suture techniques. To obtain a line of interrupted sutures free of puckering, irregularity or distortion, the following conditions must be met (see Fig. 4.17):

1. each suture must cross the suture line at $90^{\circ}$;

2. all intervals between sutures should be of the same length;

3. the bite of tissue incorporated in each half of each suture should be of the same size; and

4. all sutures should be tied with the same degree of tension. 


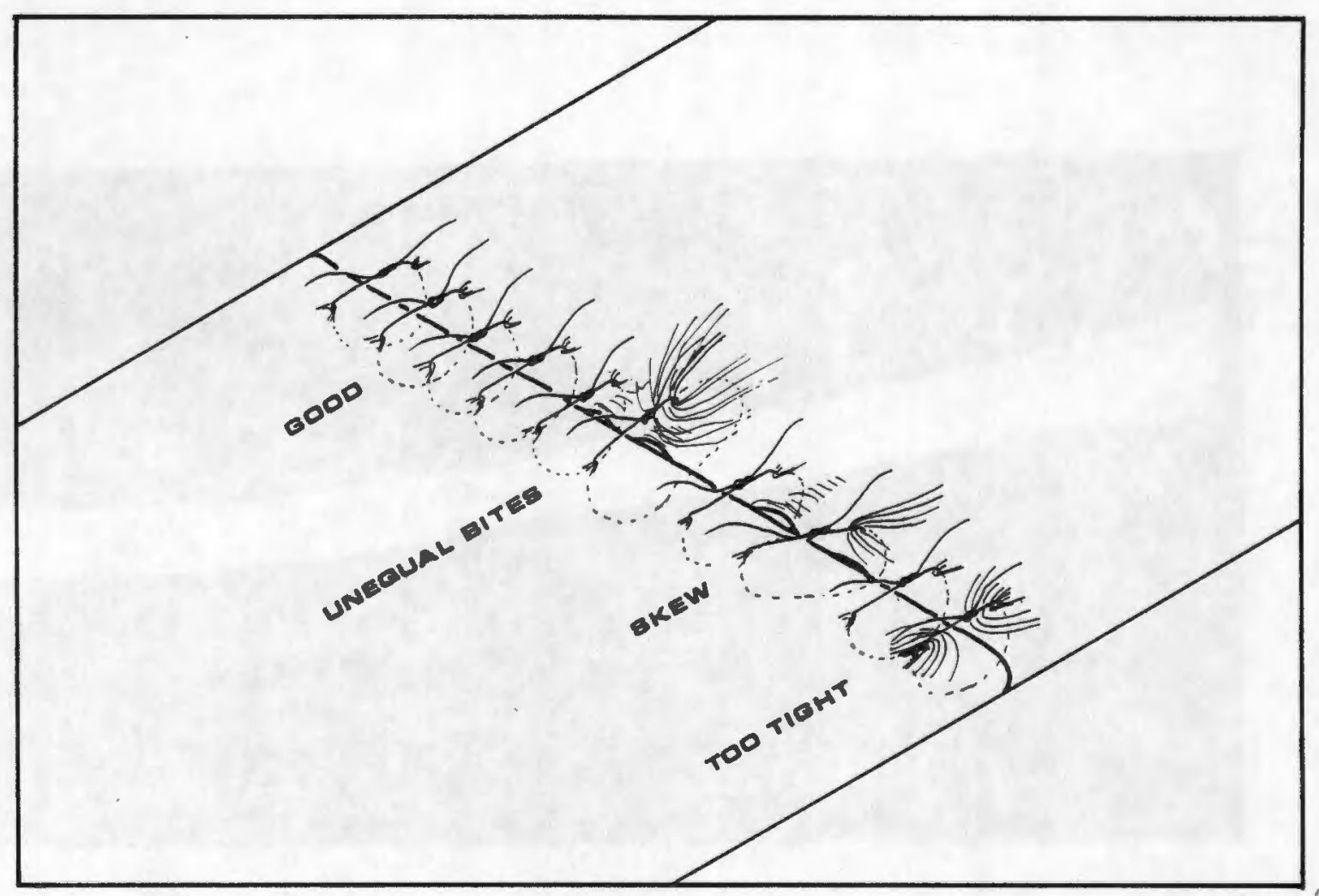

FIGURE 4.17

Causes of distortion in a line of interrupted sutures.

Most of these relatively stringent requirements are avoided by the EIEA and distortion in this procedure will generally result only if gross errors have been made. Such errors are:

1. A large difference in the lengths of the loops.

2. Taking much too large a bite of tissue during any of the FV suture passes.

The first error results in a "skewing" of the vessel, which distorts the vessel in the region of the anastomosis into a "lazy-S" shape. I have used this method of "skewing" to anastomose vessels of differing diameters and it caused no noticeable obstruction to flow through the anastomosis (see: vessels of dissimilar sizes).

The second error will result in puckering of the FV and consequent disturbance of the laminarflow pattern with eddy-currents generated by the interaction of the blood flow with the irregularities in the vessel's surface. It should also be noted that a suture, taking a large enough 
piece of tissue to cause puckering of the vessel wall, may well have penetrated the entire wall thickness, thus unnecessarily damaging the intima of the FV.

\section{MODIFICATIONS FOR OTHER VESSELS}

I have found certain modifications to be useful in the execution of the new EIEA, when employing the method in vessels other than the $1 \mathrm{~mm}$ artery for which it was originally developed.

\section{STANDARD MICROVEINS (1-2MM)}

When anastomosing veins by means of the EIEA method, it is judicious to spend a few seconds before placing any sutures to ensure that the FV and RV are correctly identified!

Rat veins are extremely thin-walled and it is therefore difficult to take a "superficial tangential bite" of the FV. I take longitudinal bites of the FV to ensure that I have an adequate grip on the wall to hold the vessels in their invaginated position. The overlap length should follow the same rule as for the arteries i.e 1.5 vessel diameters. Veins are capable of marked spasm and they should be mechanically dilated prior to anastomosis.

Small human veins are very similar in their consistency to rat arteries and they are therefore weil suited to EIEA by the standard method.

\section{VERY SMALL VESSELS $(<0.4 M M)$}

When anastomosing very small vessels, there are two problem areas to contend with. The vessel stretching forceps will often be too large to enter the lumen and the suture needles may be too big. I dilate the vessel by first using the polished-needle cannula in its "hydrodilation" mode (see Appendix A). This usually dilates the vessel enough for the smallest vessel 
stretching forceps (ST\&D size 5az) to gain entry. I use an 11/0 monofilament Nylon suture on a $303 \mathrm{~mm}$ straight needle (Ethicon ST30-3) to make the anastomosis.

\section{SMALL VESSELS (2-3MM)}

In these vessels, a $7 / 0$ or $8 / 0$ suture is usually appropriate. I use two double-armed sutures (Ethicon TM B-701) and start the loops by taking superficial transverse tangential bites of the surface of the FV at the points where the sutures would normally pass through the FV. The needles are then either held parallel in the needle holder and passed through the wall of the RV from inside to outside in the appropriate positions in one pass, or they are held individually and passed through the wall in separate passes. In either case the end result looks exactly the same as that of the normal technique. The availability of larger double-armed sutures at a reasonable price makes this method, which is quicker and easier than the standard method, an attractive alternative for larger vessels.

\section{VASCULAR INTERPOSITION GRAFTS}

Many reconstructive procedures require the employment of interposition grafts to gain adequate vessel length $(11,42,70,49,117,136,148,160,168,179,212)$. The use of an interposition graft requires the employment of two anastomoses in every vessel which is repaired and it would therefore be advantageous to use the EIEA, which takes much less time to perform than an ETEA.

\section{AUTOLOGOUS VEIN}

Lauritzen has pointed out that a sleeve anastomosis should not be used at the downstream end of an autologous vein interposition graft. He says that the arterial pressure causes the vein to bulge at the point of entry into the RV and then to produce a valve-like effect, which may lead to occlusion of the vessel. The EIEA may, however, be used with good effect at the upstream or feeding end of the graft $(110,116)$. Vilkki (197) agrees with these findings. 
Having been cautioned by this work, I have not used the EIEA in the downstream (Graft-to-RV) anastomosis, but I have routinely used it in the FV-to-Graft anastomosis. 1

\section{SYNTHETIC MATERIAL}

The development of synthetic materials, which could be fashioned into tubular structures and which could then be effectively used as microvascular interposition grafts with the same degree of success as autologous vein grafts, would be an enormous contribution to the field of microvascular surgery. Such materials have been used extensively in vascular surgery and they have also been employed successfully in microvascular surgery $(49,148)$.

Lauritzen has reported on the use of absorbable Polyglycolic acid (PGA) grafts in microvessels in which he also used sleeve anastomoses (117). In this situation he elected to use a normal sleeve anastomosis at the Graft-RV end and a reversed sleeve anastomosis at the FV-Graft end! Six out of his 12 venous grafts were patent at follow-up examination, but all the arterial grafts were occluded. It must be added that the material of which the tubes were made, was very rigid and impossible to penetrate with microneedles. The vessels which remained patent under these adverse conditions, had developed a near-normal pseudo-intima, which was encouraging.

I have not had the opportunity to use the EIEA in microvascular synthetic grafts, but I have used it on six occasions in the reconstruction of much larger human vessels by means of Poly-tetrafluoro-ethylene (PTFE) "Goretex" grafts. In these cases I have used the double-armed suture method suggested for small vessels. The graft material, selected from those available in the theatre, was always the smallest one which would fit over the outside of the vessel. The EIEA was used for the FV-Graft anastomosis and an ETEA was used at the Graft-RV end. Two femoral arteries, 3 popliteal arteries and 1 brachial artery were repaired in this fashion. All the patients had good distal pulses and dry anastomoses before wound closure. One patient had a second popliteal artery graft performed in the same way after signs of distal ischaemia on the second post-operative day. At operation it was found that the vessel had not been adequately debrided and a traumatised area distal to the graft had become occluded. 


\section{VESSELS OF DISSIMILAR SIZES}

The new EIEA makes it possible to join vessels of dissimilar sizes within reasonable limits. This is achieved by varying the loop lengths as shown in Fig. 3.19. It is unusual in standard practice to meet situations where it may become necessary to join vessels with a very large difference in their sizes and it would definitely not be possible to use an EIEA when the FV is much larger than the RV. However, as Lauritzen points out, such a situation also causes difficulty with the ETEA (115).

\section{ARTERIOVENOUS FISTULA CONSTRUCTION}

Nordgren and Cohen (142) have used the Lauritzen sleeve anastomosis (112) to create arteriovenous shunts in the dog. They anastomosed the radial artery to a cutaneous vein and achieved 10/12 (83\%) patency at 1 week and 9/12 (75\%) at 1 month. Their operations took an average time of 17 minutes (skin-to-skin without an assistant).

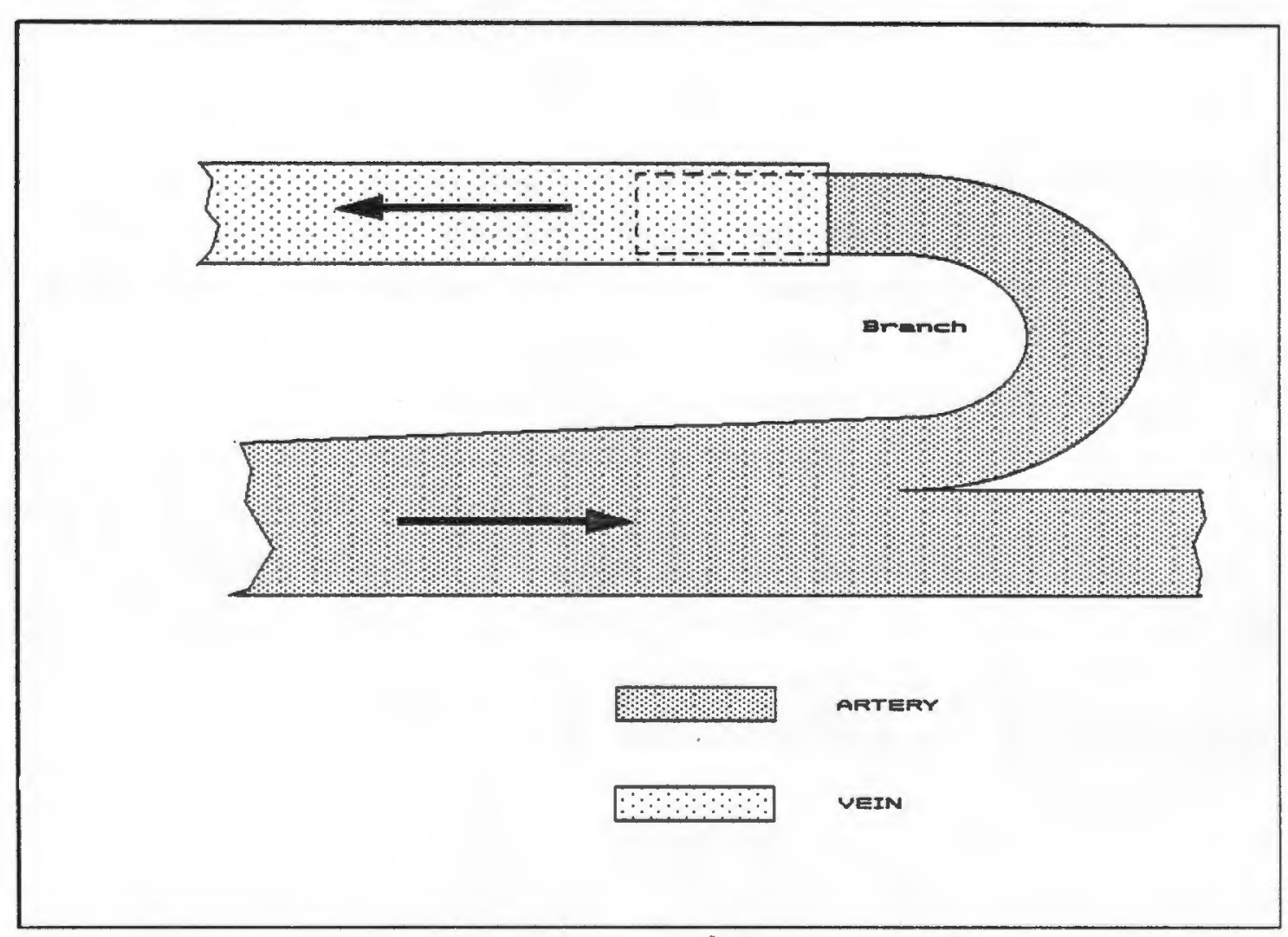

FIGURE 4.18

Arteriovenous fistula construction using the EIEA. 
The new EIEA is now being tested in arteriovenous shunts in pigs and it should soon be available for use in patients requiring haemodialysis.

The only modification which may be required, is that of "skewing", if there is a significant difference between the sizes of the feeding artery and the receiving vein.

\section{NON-VASCULAR TUBULAR STRUCTURES}

Reconstitution of the bile duct has long been a problem area in liver transplantation models in the rat. Sun Lee implanted the donor choledochus into the recipient duodenum (119), but other workers found that this resulted in necrosis of the duodenal wall and subsequent peritonitis. Kamada uses a cuff technique, in which the donor duct is everted over a piece of plastic tubing and then invaginated into the recipient's duct (97). This procedure requires the availability of a considerable extra length of bile duct and introduces a large piece of foreign material into the vicinity of the duct.

Fronticelli et al (65) have joined the bile duct in the rat by means of a telescoped anastomosis, which was a modification of the Meyer technique (133). They reported a $100 \%$ success rate in 20 animals, evaluated at 10,20 and 30 days post surgery with no bile leakage, no impairment of liver function and no stenosis of the duct. They stress the importance of minimising the number of sutures, as granulomatous reactions occur readily around sutures in the bile duct. Such reactions may lead to scarring and cicatrisation or to mural necrosis and leakage of bile. Their good results were achieved in spite of the fact that their modification of Meyer's technique caused exposed suture material to be present in the lumen of the duct.

The advantages of the new EIEA over the Meyer technique have already been discussed. I have used the new EIEA in a limited study, anastomosing the bile ducts of 5 rats. There were no technical problems and all the animals survived a full month before being sacrificed. At necropsy there were no macroscopic signs of biliary obstruction, hepatic failure or leakage from the bile duct in any of the animals.

The success of the EIEA in the rat's bile duct, which is not a high pressure system, may indicate that it could also be useful in the reconstruction of lymphatic vessels which have to date been difficult to anastomose, despite the use of microsurgical methods (18). 
Chapter 4 - Surgical Techniques for the EIEA

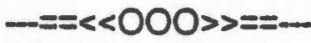

\section{NOTES}

1. No failures in 16 cases; all followed for $>3 / 12$. 


\section{CHAPTER V. \\ EVALUATION OF THE NEW EIEA}

Nothing can be loved or hated unless it is first known.

Leonardo da Vinci (c.1500)

\section{INTRODUCTION}

Evaluation of the EIEA initially took the form of a feasibility study, followed by a range of tests in different situations and comparisons with other techniques.

In the cases where vessels other than those of rats were used, the number of procedures was often too small for statistical evaluation. The descriptions of these limited studies have however been included as they may form the bases for further studies of the EIEA.

In some areas new techniques for evaluating microvascular anastomoses were developed. The most significant of these developments is the use of corrosion casts combined with photomicrography to make detailed and exact examinations and measurements of the vessel lumen in the vicinity of the anastomosis.

Some of the individual experiments described below, were performed concurrently to save on operating materials and on the number of animals used. In some cases exactly similar parts of different experiments were grouped for the purpose of analysing single aspects of the EIEA. The experiments have been grouped for the sake of clarity and are thus not in chronological order.

All the animals used, were housed in standard cages, which were cleaned and lined with fresh litter at least once per day. They were fed ad libitum with species-specific food cubes and fresh tap water was available at all times. General anaésthetics were administered for all operative procedures and no animal experienced more than one postoperative period. 


\section{THE EIEA IN DIFFERENT VESSEL SIZES \& TYPES}

\section{INITIAL TESTING IN RAT FEMORAL ARTERIES}

\section{AlMS}

The first series of new EIEA's was performed in rat femoral arteries to determine whether:

1. It was a practicable procedure.

2. It was consistently practicable.

3. The vessel remained patent.

4. There were faults or potential pitfalls which needed correction.

\section{METHODS AND MATERIALS}

The initial part of the investigation was carried out in the same way as the earlier evaluations of the Lauritzen, Meyer and Hyland methods. These animals were, however, re-evaluated at 7-14 days to determine patency of the anastomoses.

Twenty adult male Sprague-Dawley rats (mass $300 \mathrm{~g}-400 \mathrm{~g}$ ) were anaesthetised by means of an intraperitoneal injection of Ketalar $10 \mathrm{mg} / 100 \mathrm{~g}$ and Acepromazine $0.5 \mathrm{mg} / 100 \mathrm{~g}$. Their lower abdomen and groin areas were shaved and cleaned with a Povidone lodine solution. They were placed in a supine position on a firm surface and held in position by means of soft limb restraints.

The femoral neurovascular bundle was exposed by means of a curved groin incision and lateral reflection of the fat pad. The femoral artery was dissected free by a combination of 
hydrodissection (Appendix A) and sharp dissection. The anastomosis was carried out in the first 14 cases by the standard procedure for the new EIEA. In the last 6 cases deliberate deviations were introduced to observe their effects. All anastomoses were observed for 30 minutes before the wounds were washed with a 1:100 Povidone lodine solution in Normal Saline and closed with continuous sutures of $4 / 0$ chromic catgut.

The progress of each operation was carefully noted.

After 7-14 days the animals were re-anaesthetised and the femoral arteries were examined and tested for patency. A lethal dose of $\mathrm{KCl}$ solution was administered intravenously while the animal was still under the same anaesthetic.

\section{RESULTS}

The tabulated results are displayed in Fig. 5.1.

\begin{tabular}{|c|c|c|c|c|c|c|}
\hline $\begin{array}{l}\text { CASE } \\
\text { no. }\end{array}$ & $\begin{array}{l}\text { SUTURE } \\
\text { SEPARTH } \\
\text { ANGLE } \\
\text { (OEG) }\end{array}$ & $\begin{array}{l}\text { OYER- } \\
\text { LAP } \\
(O|A|\end{array}$ & $\begin{array}{c}\text { RESULT } \\
\text { IHHEOIATE }\end{array}$ & $\begin{array}{l}\text { RESULT } \\
30 \text { HIN }\end{array}$ & $\begin{array}{l}\text { Posi } \\
\text { Oper } \\
\text { OAYS }\end{array}$ & Pateacy \\
\hline $\begin{array}{l}1 \\
2 \\
3 \\
1 \\
5 \\
8 \\
7 \\
8 \\
9 \\
10 \\
11 \\
12 \\
13 \\
11\end{array}$ & $\begin{array}{l}170-180 \\
175-185 \\
160-170 \\
175-185 \\
170-180 \\
180-170 \\
175-185 \\
170-180 \\
170-180 \\
185-175 \\
175-185 \\
180-170 \\
170-180 \\
165-175\end{array}$ & $\begin{array}{l}1.8 \\
2.0 \\
1.8 \\
1.8 \\
1.8 \\
2.0 \\
1.8 \\
1.8 \\
2.0 \\
1.8 \\
1.8 \\
1.8 \\
1.8 \\
1.0\end{array}$ & 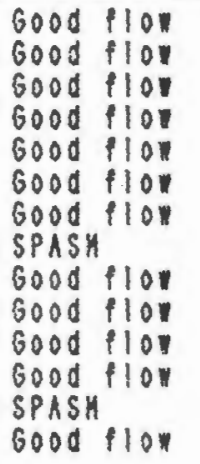 & 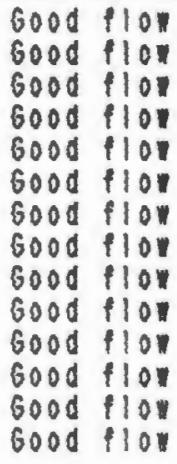 & $\begin{array}{r}8 \\
9 \\
8 \\
10 \\
7 \\
8 \\
11 \\
13 \\
7 \\
9 \\
8 \\
8 \\
9 \\
10\end{array}$ & 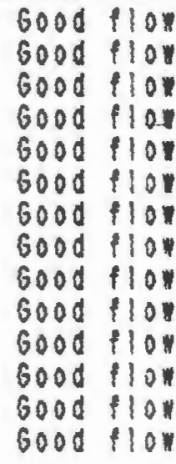 \\
\hline $\begin{array}{l}15 \\
16 \\
17 \\
18 \\
19 \\
20\end{array}$ & $\begin{array}{l}135-145 \\
120-130 \\
170-180 \\
175-185 \\
155-175 \\
170-180\end{array}$ & $\begin{array}{l}1.8 \\
1.8 \\
1.0 \\
0.8 \\
0.8 \\
3.0\end{array}$ & $\begin{array}{l}\text { 6ood flo: } \\
\text { Good flo } \\
\text { Good } 110 \% \\
\text { GoOd flo } \\
\text { LEAK } \\
\text { SPASH }\end{array}$ & $\begin{array}{l}\text { Good floy } \\
\text { Good floy } \\
\text { Good flo } \\
\text { Good flow } \\
\text { Good flou } \\
\text { POOR FloY }\end{array}$ & $\begin{array}{r}12 \\
9 \\
8 \\
9 \\
9 \\
8\end{array}$ & 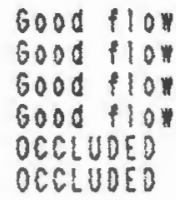 \\
\hline
\end{tabular}

FIGURE 5.1

Results of new EIEA in rat femoral artery. 
All sutures were easily placed without any entwinements. Invagination was automatic and uncomplicated in all cases.

Excessive downstream spasm occurred in 3 cases. Two of these occurred in the first 14 (No.8 \& No.13) and their spasms were relieved by the application of a few drops of $2 \%$ Xylocaine solution. Flow through these two vessels appeared as good as that in any of the other vessels at $30 \mathrm{~min}$. In the third case (No.20), which was telescoped to a depth of 3 vessel diameters, flow remained poor after relief of the spasm and the vessel was found to be occluded at follow-up on the 8th postoperative day.

In case No.19, where a very short overlap was deliberately used, the anastomosis leaked excessively before settling down to apparent normality. This vessel was, however, also occluded at follow-up on the 9th day.

\section{DISCUSSION}

The new EIEA proved to be easy to perform. There were no suture entwinements in the twenty anastomoses and invagination was very simple to effect in every case. Unlike the previous investigations of other techniques, this evaluation contained a follow-up examination of each of the anastomoses. This follow-up was performed in the second postoperative week as recommended by Hayhurst and O'Brien (78), who found that patency rates were falsely elevated after the second week due to the development of collateral vessels around the anastomotic site. Only two vessels were found to be occluded at follow-up and both of these were in the "deliberate error" group. It was interesting to note that the errors which resulted in occlusion of the vessels, were both related to overlap length, one being too short ( $0.4 \mathrm{X}$ diameter) and the other too long ( $3.0 X$ diameter). In the case with the long overlap the vessel was also in spasm immediately after clamp release. I thought that this spasm might have been due to the fact that the vessel had been stretched longitudinally to obtain enough length for the additional overlap. Spasm after clamp release occurred in two other cases (Nos. 8 \& 13). In both of these the vessel had not been dissected free to well beyond the edges of the clamps. The additional length required for the overlap was thus achieved by stretching a short length of vessel, i.e. the elongation per unit of natural length was more than it would have been had the vessel been freed over a longer distance. 
The suture separation angle did not appear to be critical, as demonstrated by No.16 $\left(120^{\circ}\right.$. $130 \%$ ).

\title{
SUMMARY
}

A pilot study was performed to evaluate the new EIEA in practice and to identify pitfalls in the method. The results indicated that the EIEA was easy to perform and that there were very few pitfalls in the technique. It was noted that both excessively long and very short overlaps were to be avoided. An overlap length of 1-2 vessel diameters, as recommended by others who have used the sleeve principle $(131,108,133,87)$, gave the desired results. The EIEA tolerated large variations in the suture separation angle.

\section{STANDARD MICROVESSELS}

\begin{abstract}
AlMS
I have grouped the results of a number of experiments performed on standard arteries of $1 \mathrm{~mm}$ in diameter under this heading. The aim is to look at the performance of the new EIEA in isolation, using the parameters of operating time and patency rates.
\end{abstract}

\section{METHODS AND MATERIALS}

The anastomoses analysed here are a combination of the 97 EIEA's performed in my own comparative studies and the 37 EIEA,s performed by students of microsurgery.

The methods and materials used, are fully described in the relevant sections. 


\section{RESULTS}

\section{Time Taken}

All 97 of the anastomoses performed by me were carefully timed, as were 14 of those performed by the students. When the F-test was applied to these data it was obvious that they were not homogeneous $\left(F=178 ; d_{W}=109 ; d f_{B}=1-P<0.05\right)$. When the F-test was applied to my own data, divided into 11 groups of 8 and 1 group of 9 , the data, were also not homogeneous $\left(F=15.72 ; d_{W}=85 ; d f_{B}=11-P<0.01\right)$. However, when the first group of eight anastomoses, which were performed while I was developing and learning the technique, was discarded, the remaining 89 data were homogeneous $\left(F=1.54 ; d_{W}=78 ; d_{B}=10-P>0.05\right)$. The analysis of these data is tabulated in Fig. 5.2.

\begin{tabular}{|c|c|c|c|c|}
\hline $\begin{array}{l}\text { MAXIMUM } \\
\text { min.sec }\end{array}$ & $\begin{array}{c}\text { MINIMUM } \\
\text { min.sec }\end{array}$ & $\begin{array}{c}\text { MEAN } \\
\text { min.sec }\end{array}$ & $\begin{array}{c}\text { STAN.DEV } \\
\text { min.sec }\end{array}$ & $\begin{array}{c}\text { VARIANÇE } \\
\text { min) }\end{array}$ \\
\hline 13.30 & 5.27 & 7.36 & 1.37 & 2.60 \\
\hline
\end{tabular}

FIGURE 5.2

Analysis of operating times in 89 ElEAs.

The distribution of these times is graphically represented in Fig. 5.3. 


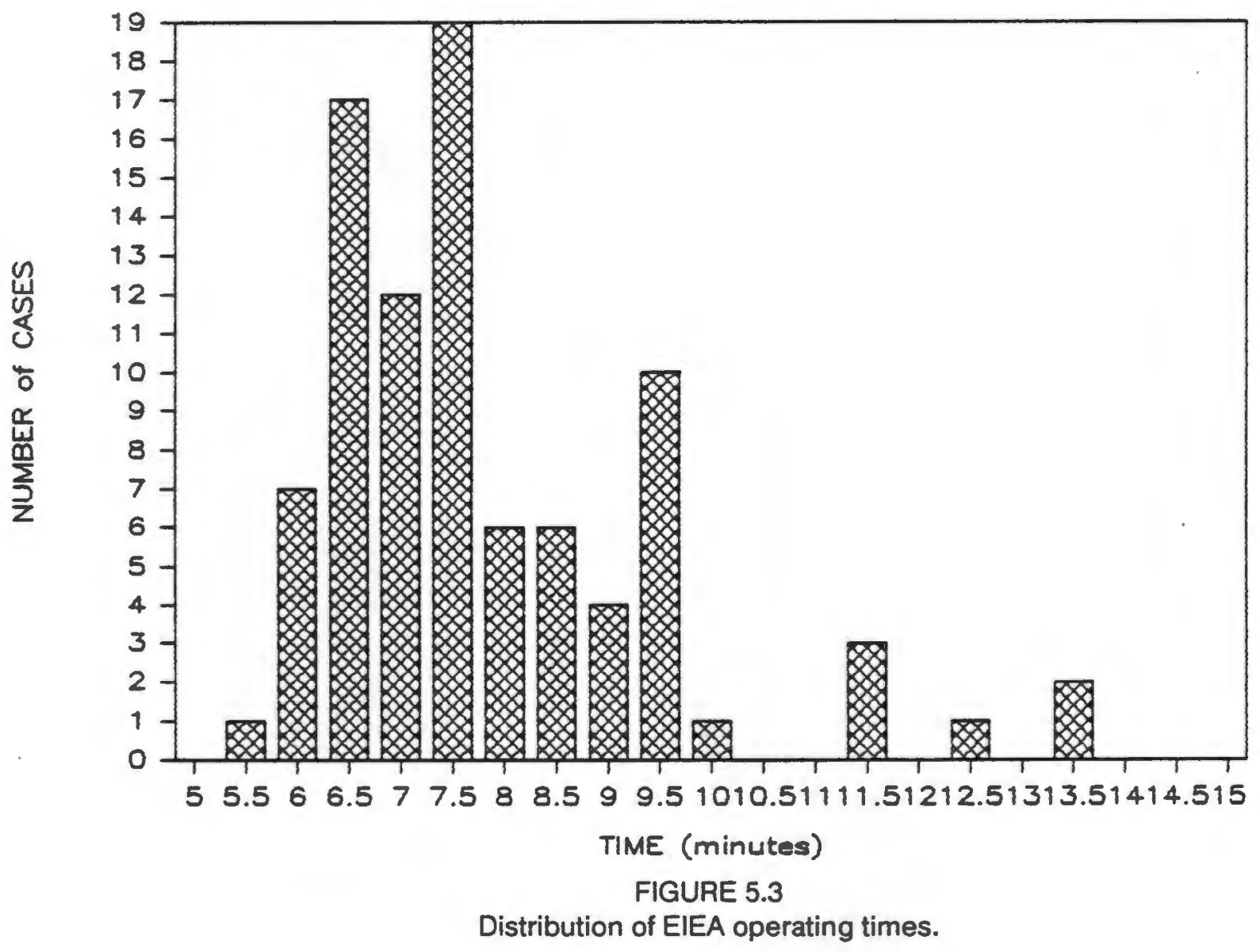

When the operating time data of my first group of 8 anastomoses were grouped with the data of the 14 timed operations performed by the novice microsurgeons, the data were reasonably homogeneous $\left(F=5.08 ; d_{W}=20 ; d_{B}=1-0.05>P>0.01\right)$. The analysis of these data is tabulated in Fig. 5.4 and the distribution is shown in Fig. 5.5. 


\begin{tabular}{|c|c|c|c|c|}
\hline $\begin{array}{c}\text { MAXIMUM } \\
\text { min }\end{array}$ & $\begin{array}{c}\text { MINIMUM } \\
\text { min }\end{array}$ & $\begin{array}{c}\text { MEAN } \\
\text { min.sec }\end{array}$ & $\begin{array}{c}\text { STAN.DEV } \\
\text { min.sec }\end{array}$ & $\begin{array}{c}\text { VARIANÇE } \\
\text { (min) }\end{array}$ \\
\hline \hline 46 & 11 & 23.24 & 8.54 & 79.15 \\
\hline
\end{tabular}

FIGURE 5.4

Analysis of EIEA operating times by novice microsurgeons.

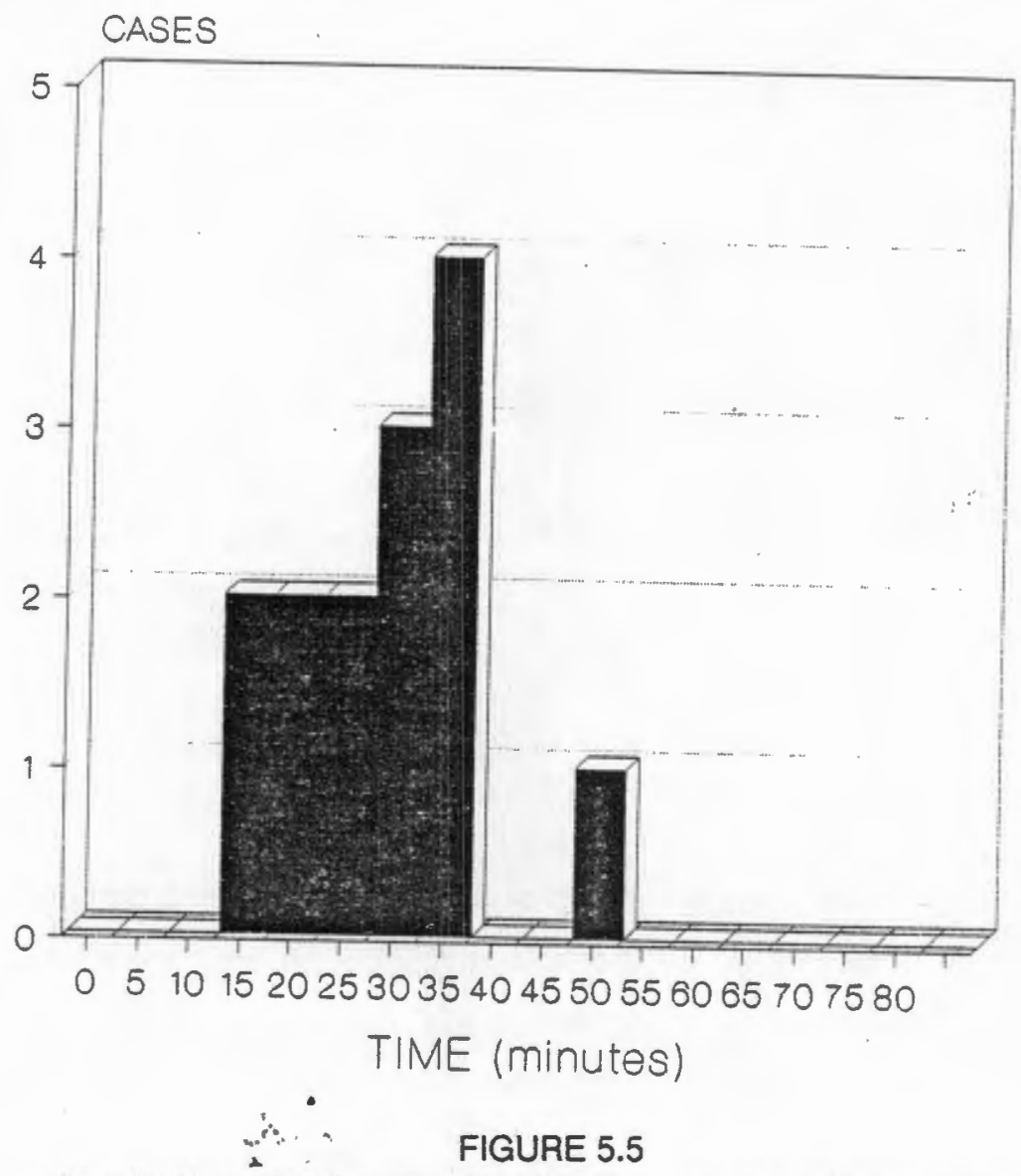

Distribution of EIEA operating times by novice microsurgeons.

\section{Patency}

Four of the 97 animals on which I performed the new EIEA were lost to follow-up (3 died, 1 lost label possibly misread). There was 1 occluded vessel in the remaining 93 animals. Two of the 37 animals which had had the new EIEA performed by students of microsurgery, had occluded vessels. The difference in patency rates between these groups was not significant $\left(X^{2}=2.03\right.$; $d f=1-P>0.05)$. 
The vessels were evaluated at different postoperative intervals, ranging from 0 to 50 days, with a mean evaluation time of 13.6 days. In the 130 anastomoses performed by 12 different surgeons there were 3 vessels which were occluded i.e. a patency rate of $97.7 \%$.

\section{DISCUSSION}

There was a high level of homogeneity in the operating times for the last 89 anastomoses of the group of $1 \mathrm{~mm}$ vessels. This was confirmed by the F-test and displayed in a more practical

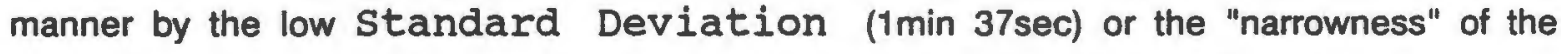
distribution curve in Fig. 5.3. I attribute the consistent nature of the operating time to the fact that once the technique has been learned, it is not prone to many unforeseen, time-consuming errors.

This group of 89 anastomoses was representative of my own operating time for the new EIEA once the procedure had been learnt. It could probably also serve as a control for most other surgeons who know this technique or any other anastomotic procedure, which they wish to compare with the new EIEA on the basis of operating time.

The practical implication of the results is that the surgeon, who has acquired and practised the EIEA technique, may expect to regularly complete an anastomosis in a $1 \mathrm{~mm}$-diameter artery in less than 10 minutes.

The analysis of the results of the "learning" group shows that even inexperienced microsurgeons are usually able to complete a $1 \mathrm{~mm}$ anastomosis in less than half an hour. As may be expected, the data were more widely distributed (Fig. 5.5). 


\begin{tabular}{|l|l|l|l|c|c||}
\hline AUTHOR & REF & $\begin{array}{l}\text { ANAST. } \\
\text { TYPE }\end{array}$ & TOTAL & PATENT & $\begin{array}{c}\text { PATENCY } \\
\text { RATE }\end{array}$ \\
\hline \hline HYLAND & 87 & EIEA & 20 & 18 & $90 \%$ \\
LAURITZEN & 109 & EIEA & 21 & 21 & $100 \%$ \\
LAURITZEN & 112 & EIEA & 39 & 39 & $100 \%$ \\
LAURITZEN & 108 & ETEA & 42 & 42 & $100 \%$ \\
LAURITZEN & 108 & EIEA & 42 & 41 & $98 \%$ \\
MAN \& ACLAND & 125 & ETEA & 20 & 17 & $85 \%$ \\
MAN \& ACLAND & 125 & ETEA & 20 & 16 & $80 \%$ \\
MERKEL & 132 & ETEA & 20 & 19 & $95 \%$ \\
MERKEL & 132 & ETEA & 20 & 20 & $100 \%$ \\
MEIER & 131 & EIEA & 12 & 10 & $83 \%$ \\
MEYER & 133 & EIEA & 80 & 74 & $93 \%$ \\
MEYER & 133 & ETEA & 80 & 74 & $93 \%$ \\
NIEUBOURGH & 141 & ETEA & 25 & 23 & $92 \%$ \\
\hline
\end{tabular}

FIGURE 5.6

Patency rates for microvascular anastomoses in other published studies.

The patency rates of both groups are most gratifying and compare favourably with those achieved in other published studies $(108,112,76,77,78)$. This is even more impressive when one takes into account the fact that more than $25 \%$ of the anastomoses in my group of 130 were performed by novices. The patency rates achieved in other studies are tabulated in Fig. 5.6.

\section{SUMMARY}

One hundred and thirty-four new EIEAs performed in $1 \mathrm{~mm}$ rat femoral arteries were evaluated by the parameters of patency and operating time. Of these anastomoses 97 were performed by me and 37 by novice microsurgeons.

Only 14 of the second group of 37 were correctly timed. The data of the first 8 anastomoses in the group of 97 had to be discarded to achieve homogeneity by the F-test criteria. The discarded 8 data, produced in my own learning phase, were homogeneous with the 14 data produced by novice microsurgeons who had been taught the technique. I thus arrived at the two sets of homogeneous operating time data, the analyses of which are tabulated in Fig. 5.7. 


\begin{tabular}{|c|c|c|c|c|c|}
\hline LEVEL & $\begin{array}{l}\text { MAXIMUM } \\
\text { min.sec }\end{array}$ & $\begin{array}{l}\text { MINIMUM } \\
\text { min.sec }\end{array}$ & $\begin{array}{c}\text { MEAN } \\
\text { min.sec }\end{array}$ & $\begin{array}{l}\text { STAN.DEV } \\
\text { min.sec }\end{array}$ & $\begin{array}{l}\text { VARIANÇE } \\
(\min )^{2}\end{array}$ \\
\hline $\begin{array}{l}\text { EXPERT } \\
\text { NOVICE }\end{array}$ & $\begin{array}{l}13.30 \\
46.00\end{array}$ & $\begin{array}{r}5.27 \\
11.00\end{array}$ & $\begin{array}{r}7.36 \\
23.24\end{array}$ & $\begin{array}{l}1.37 \\
8.54\end{array}$ & $\begin{array}{r}2.60 \\
79.15\end{array}$ \\
\hline
\end{tabular}

FIGURE 5.7

EIEA - Operating time in $1 \mathrm{~mm}$ arteries

The patency of 130 vessels $\{[97-4]+[37]\}$ was evaluated at postoperative intervals varying from 0 to 50 days. One of the 93 and 2 of the 37 vessels were occluded. The difference between these results was not significant $(P>0.05)$. The overall patency rate was therefore $97.7 \%$ and this compares favourably with that derived from the data of other authors.

\section{VERY SMALL VESSELS (<0.4 MM)}

\section{AlMS}

Vessels with a diameter of less than $0.5 \mathrm{~mm}$ are very difficult to join by standard methods. I thought that the EIEA might be a solution to the problem if I could obtain sutures, needles and instruments small enough to match the vessels.

\section{METHODS AND MATERIALS}

Initial testing was carried out in the femoral arteries of 32 young white mice (Mus musculus var. petshopensis) with a mass of $25 \mathrm{~g}-35 \mathrm{~g}$. They were placed in a small container of known mass and weighed on a laboratory scale before being anaesthetised with an intraperitoneal injection of Ketamine $(1 \mathrm{mg} / 10 \mathrm{~g})$ and Acepromazine $(0.05 \mathrm{mg} / 10 \mathrm{~g})$, positioned prone on an operating board and held with soft adhesive tape limb restraints. Their groins were shaved by means of scalpel blades and cleaned with a hibitane solution. In each case a curved, medially-convex 


\section{Chapter 5 - Evaluation of the new EIEA}

incision was made in the groin and the fat pad was reflected laterally, revealing the femoral neurovascular bundle. The animal was placed under the microscope in such a way that the RV was on my right, as this facilitated suture placement. From here onwards the microscope was used at $30 x$ to $40 x$ magnification.

The femoral artery was dissected free by means of the polished-needle cannula in its hydrodissection mode (see Appendix A.). A $2 \%$ Xylocaine solution was used as the dissecting fluid to overcome and prevent vasospasm. The vessel diameter was measured by means of the obliquely cut EKG paper method (see Fig. 3.1). A venous-strength approximating clamp set was applied to the vessel and it was divided between the clamps. The vessel ends were flushed with heparinised saline solution (25 i.u./ml) and excessive adventitia was trimmed off. The vessels were dilated by means of the polished-needle cannula and the Acland's vessel stretching forceps (ST\&D No.5az) as previously described.

An 11/0 (14 m) nylon suture on an ST30-2 (30 m diam., 2mm length) straight needle (No.2811, ETHICON, INC., 1979, Somerville, New Jersey, U.S.A.) was used for the anastomoses.

In every case an electronic stopwatch was started at 2-3 seconds before the time of vessel division and stopped again within 2-3 seconds after the final clamp release. The elapsed time was converted to decimal figures and recorded.

The inguinal fat pad was repositioned and the skin closed with a continuous subcuticular $7 / 0$ nylon suture. The animal was placed on a $37^{\circ} \mathrm{C}$ warming pad to recover from the anaesthetic.

Numbers were drawn from a box to determine follow-up times. The selected animals were weighed, anaesthetised and prepared as described for the first operation. The femoral arteries were again dissected free and the diameter measured by the same method after vasospasm had been overcome by the application of $2 \%$ Xylocaine solution. Patency of the vessels was assessed, firstly by observing "wriggling" of the superficial epigastric artery distal to the anastomosis (1), secondly by the so-called "milking test" (78) and finally by observing active pulsatile bleeding from the vessel which had been cut distal to the anastomosis. A vessel segment containing the anastomosis was removed and preserved in $10 \%$ formaldeyhde solution for further examination. The animal, still deeply anaesthetised, was killed by an intravenous injection of $\mathrm{KOH}$ solution via the femoral vein. 
Chapter 5 - Evaluation of the new EIEA

\section{RESULTS}

Fig. 5.8 shows a tabulation of the raw data obtained from this study, analysis of the data follows overleaf.

\begin{tabular}{|c|c|c|c|c|c|c|c|c|c|}
\hline $\begin{array}{c}\text { CASE } \\
\text { NO }\end{array}$ & $\begin{array}{c}\text { BODY } \\
\text { MASS } \\
\text { (gm) }\end{array}$ & $\begin{array}{l}\text { VESS } \\
\text { DIAM } \\
(\mathrm{mm})\end{array}$ & $\begin{array}{l}\text { TIME } \\
\text { TAKEN } \\
\text { (min) }\end{array}$ & $\begin{array}{c}\text { FAT } \\
0 \\
\text { MIN }\end{array}$ & $\begin{array}{r}\text { FAT } \\
15 \\
\text { MIN }\end{array}$ & $\begin{array}{c}\text { FOLLOW } \\
\text { UF } \\
\text { (days) }\end{array}$ & $\begin{array}{l}\text { EODY } \\
\text { MASS } \\
\text { (gm) }\end{array}$ & $\begin{array}{l}\text { VESS } \\
\text { DIAM } \\
(\mathrm{mm})\end{array}$ & $\begin{array}{l}\text { F'AT } \\
\text { LATE }\end{array}$ \\
\hline 1 & 26.6 & 0.15 & 11.1 & $Y$ & $Y$ & 9 & 29.6 & 0.17 & Y \\
\hline 2 & 39.2 & 0.25 & 6.4 & $Y$ & $Y$ & 23 & 49.7 & 0.34 & $Y$ \\
\hline 3 & 29.6 & 0.18 & 8.8 & $Y$ & $Y$ & 8 & 32.5 & 0.21 & $Y$ \\
\hline 4 & 35.2 & 0.22 & 7.4 & $Y$ & $Y$ & 7 & 38.1 & 0.26 & Y \\
\hline 5 & 28.4 & 0.17 & 9.7 & Y & $Y$ & 13 & 3.0 & 0.20 & Y \\
\hline 6 & 23.5 & 0.12 & 12.7 & NO & $Y$ & 1 & 23.8 & 0.12 & Y \\
\hline 7 & 25.4 & 0.15 & 11.4 & $Y$ & $Y$ & 16 & 30.5 & 0.17 & NO \\
\hline 8 & 29.8 & 0.18 & 9.1 & $Y$ & $Y$ & 12 & 34.2 & 0.22 & $Y$ \\
\hline 9 & 2.2 & 0.20 & 8. 1 & Y & $Y$ & 15 & 38.0 & 0.24 & $Y$ \\
\hline 10 & 26.4 & 0.16 & 16.7 & $Y$ & NO & 9 & 29.4 & 0.17 & NO \\
\hline 11 & 31.6 & 0.17 & 9.2 & $Y$ & $Y$ & 42 & 48.2 & 0.30 & $Y$ \\
\hline 12 & 21.7 & 0.12 & 14.4 & $Y$ & $Y$ & 2 & 22. & 0.14 & $Y$ \\
\hline 13 & 25.8 & 0.15 & 11.2 & Y & $Y$ & 7 & 28.1 & 0.17 & $Y$ \\
\hline 14 & 3.5 .2 & 0.20 & 8.1 & $Y$ & $Y$ & 9 & 36.8 & 0.24 & $Y$ \\
\hline 15 & 24.6 & 0.14 & 11.9 & ND & $Y$ & 15 & 29.3 & 0.15 & NO \\
\hline 16 & 25.8 & 0.15 & 11.0 & Y & Y & 19 & 32.0 & 0.20 & $Y$ \\
\hline 17 & 28.4 & 0.17 & 9.7 & Y & $Y$ & 12 & 32.6 & 0.19 & Y \\
\hline 18 & 33.8 & 0.21 & 7.7 & Y & $Y$ & 9 & 5.4 & 0.24 & Y \\
\hline 19 & 22.8 & 0.13 & 13.2 & $Y$ & Y & 24 & 30.0 & 0.16 & NO \\
\hline 20 & 36.0 & 0.22 & 7.5 & $Y$ & $Y$ & 16 & 42.8 & 0.27 & $Y$ \\
\hline 21 & 27.4 & 0.16 & 10.2 & $Y$ & $Y$ & 23 & 35.3 & 0.22 & $Y$ \\
\hline 22 & 25.6 & 0.15 & 11.0 & NO & $Y$ & 19 & 31.8 & 0.18 & Y \\
\hline 23 & 40.2 & 0.28 & 5.7 & $Y$ & $Y$ & 58 & 51.2 & 0.36 & $Y$ \\
\hline 24 & 22.4 & 0.12 & $1 \Xi .3$ & $Y$ & $Y$ & 102 & 49.7 & 0.27 & Y \\
\hline 25 & 32.2 & 0.20 & 14.6 & $Y$ & $Y$ & 6 & 34.5 & 0.20 & NO \\
\hline 26 & 28.4 & 0.17 & 9.7 & $Y$ & $Y$ & 15 & 3.7 & 0.21 & Y \\
\hline 27 & 55.2 & 0.22 & 7.4 & $Y$ & $Y$ & 11 & 39.8 & 0.25 & Y \\
\hline 28 & 29.5 & 0.18 & 9.2 & $Y$ & $Y$ & 8 & 32.4 & 0.22 & Y \\
\hline 29 & 32.6 & 0.20 & 8.1 & $Y$ & $Y$ & 13 & 37.7 & 0.23 & $Y$ \\
\hline
\end{tabular}

FIGURE 5.8

Raw data for the EIEA in mouse femoral arteries.

Three animals were lost to follow-up. Two died during the hour after operation, probably as a result of an anaesthetic overdose. At necropsy neither of them was found to have lost blood from the anastomotic site. The third was claimed as a pet by my 2 year-old daughter, who bluntly 
refused a murine substitute and it died 13 months later under circumstances which precluded necropsy.

\section{Analysis of Results}

\section{Vessel Diameters}

The mean vessel diameter in this study was $0.18 \mathrm{~mm}$ (Stan.Dev. $=0.04 \mathrm{~mm}$ ). The distribution of vessel diameters is shown in Fig. 5.9.

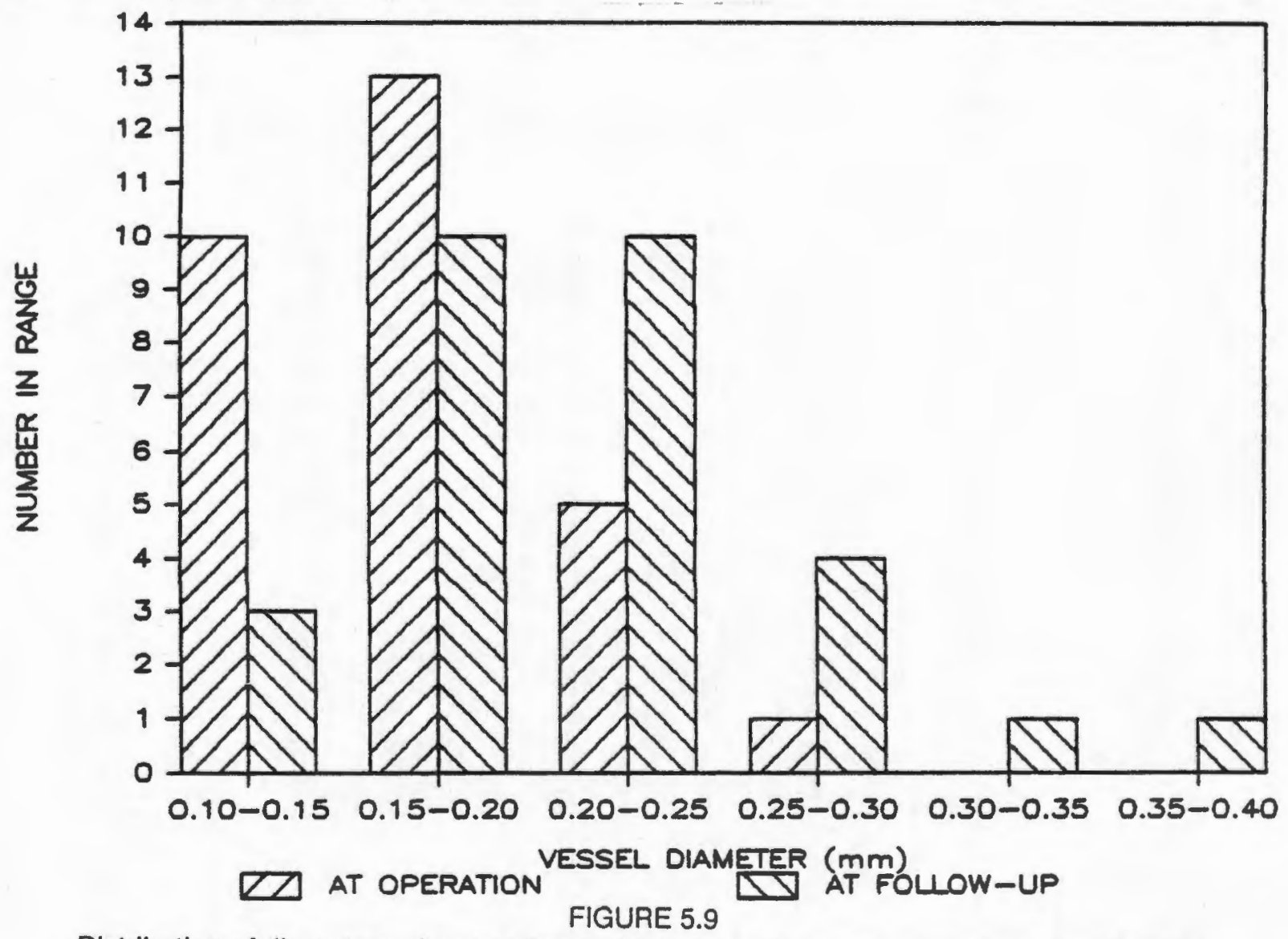

Distribution of diameters of mouse femoral arteries which were reconstituted by the new EIEA. 


\section{Time Taken}

The average time taken for an anastomosis was $10 \mathrm{~min} 09 \mathrm{sec}$ (Stan.Dev.=2min 36sec)

The graph in Fig. 5.10 shows the relationship between vessel diameter and the time taken for the anastomosis. There are two outlying points corresponding to :

1. Case \#10, in which the animal had received an overdose of anaesthetic and required resuscitation during the operation; and

2. case \#25 in which a retractor had slipped, thus dislodging the first loop before it had been tied and necessitating replacement of the suture.

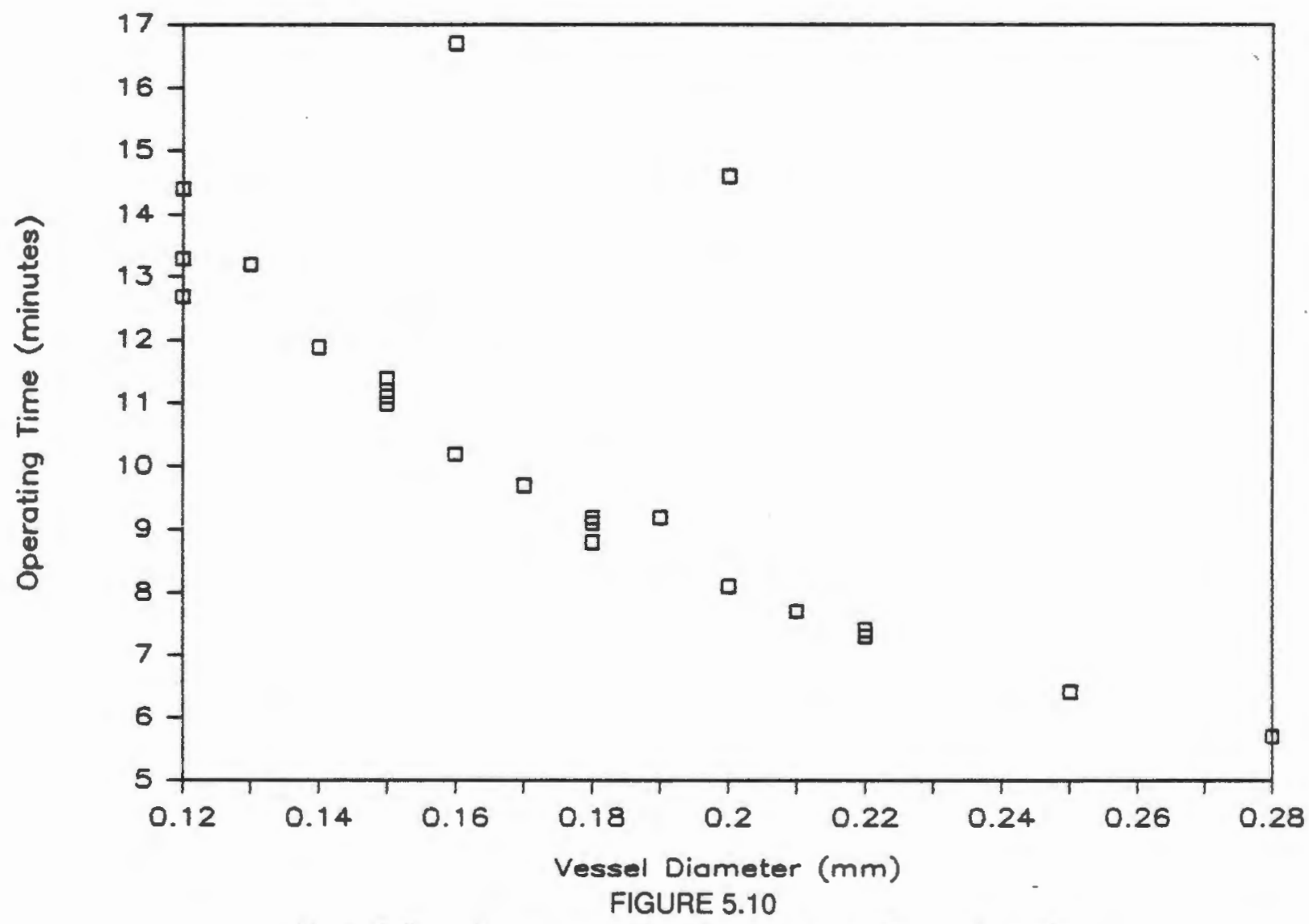

Vessel diameter vs. operating time in mouse femoral arteries.

Statistical analysis shows a high degree of negative co-variability between the vessel diameter and the time taken for the anastomosis $(r=-0.778$ therefore $p<0.001)$. If the two outlying results 
are removed for the purpose of analysis, the correlation coefficient is very much higher $(r=-$ 0.965).

It is interesting to note that if the $\log _{10}$ of the operating time is used in the calculation of the coefficient of correlation ( $r$ ), then $r=-0.989$. This indicates that there is an even higher correlation between exponential operating-time increase and vessel diameter. A vessel which is only slightly smaller than another may therefore take disproportionately longer to anastomose.

\section{Failures}

Five vessels were occluded at follow-up (17.2\% of the total). In these cases the average vessel diameter had been $0.156 \mathrm{~mm}$ and the average time for completion of the anastomosis $13 \mathrm{~min}$ 34sec.

\section{General Interest}

Statistics of general interest which could be derived from the measurements made in this study were:

1. There is a close correlation between vessel diameter and body mass in the young growing mouse. $(r=0.991)$. This correlation is less close after anastomosis of the vessel $(r=0.956)$.

2. The average daily mass increase of the animals was $1.22 \%$ of their body mass. Animals which were evaluated early, were noted to have lost mass in the early postoperative period.

\section{DISCUSSION}

The anastomosis of very small vessels by means of the new EIEA was easier than I had expected it to be. It required the use of a very small needle and high magnification, but no special instrumentation was called for and the time taken for the anastomosis was not much greater than that required for a $1 \mathrm{~mm}$ vessel. 
Huang, Chow and Chan reported on their experience with the anastomosis of 48 murine saphenous arteries in 1982 (85). They believed that there would soon be clinical applications for the anastomosis of vessels of this size and therefore wished to obtain experience with the handling of such very small vessels. One of the authors used a special double-handed, balloonoperated needle holder to perform the anastomoses, whilst another used a specially modified No. 5 Jeweler's forceps as a needle holder. Four vessels were excluded from the study and in the remaining 44 they attained an overall $77 \%$ patency rate at $1-2$ weeks, but also pointed out that in the group of cases in which the surgeon had classed the technique as "satisfactory" a patency rate of $92 \%$ was achieved, whilst in the "non-satisfactory" group the patency rate was $67 \%$. They also related patency rate to vessel diameter and found more failures in small vessels.

My results with the EIEA compare very favourably with theirs, especially when taking into account the short operating time and the fact that no assistants or special instruments, barring the polished-needle cannula, were used.

I have not found a published study in which the correlation between the vessel diameter and the time taken for the anastomosis of very small vessels is mentioned. It is possible that this correlation may not exist in other techniques due to the increased possibility for time-consuming chance events to occur when a larger number of sutures is used for each anastomosis. The effect that chance events can have on the correlation is clearly demonstrated by the effect of removing cases \#10 \& \#25 for the purpose of statistical calculation. The fundamental reason for the strong correlation is not yet clear to me. It may be related to the increasing degree of "difficulty" with decreasing size, but it may also simply be related to the increased magnification required for smalier structures and the consequent slowing of movements. Regardless of the exact reason for the high level of correlation, the fact that it exists, indicates to me a certain simplicity in the relationship between vessel diameter and time taken and this "simplicity" is due to the nature and character of the EIEA. In the words of Lauritzen:

"In the sleeve anastomosis, simplicity is the ultimate sophistication." (114)

The loss of correlation between the body mass and vessel diameter when measured at followup, as compared to the high correlation at initial measurement, would seem to indicate that different vessels are affected differently by the "insult" of an anastomosis. It was not the purpose of this study to evaluate the effect of the anastomosis on vessel growth, but this is a point worth noting in the design of such a study. 
Chapter 5 - Evaluation of the new EIEA

\section{SUMMARY}

The new EIEA was used in 32 femoral arteries of young mice (mean diameter $=0.18 \mathrm{~mm}$ ). The average time from vessel division to final clamp release was under 11 minutes. Three animals were lost to follow-up. In the remaining 29 there were 5 occlusions i.e. $82 \%$ patency. There was a high negative correlation between the vessel diameter and the time taken for the anastomosis. The success rate is equivalent to that in another published study (85). 


\section{LARGER VESSELS (2-3 MM)}

\section{AlMS}

The aim of this limited study was to establish whether the new EIEA would be an acceptable method for the joining of larger vessels and if there were any advantages to be gained by employing the technique in such vessels. Wieslander and Rausing suggest that the sleeve anastomosis of Lauritzen (which they have evaluated) should only be used in larger vessels $(204,203)$. On the other hand, larger vessels generally have thicker walls and this implies that a larger area of the cut FV's end will lie exposed to the blood stream in the anastomosis of a larger vessel. This could increase the incidence of thrombotic occlusion at the anastomotic site. If larger vessels also inherently possess a larger wall-thickness to lumen ratio (to cope with greater pressure), the possibility of stenosis occasioned by the double wall thickness would seem to further compromise the likelihood of the EIEA functioning well in such vessels (56).

I considered the fact that satisfactory functioning of the EIEA in vessels of this magnitude might make it a useful alternative in the anastomosis of the hepatic artery, which is currently the most demanding part of the liver transplant operation performed on pigs in our laboratories.

\section{METHODS AND MATERIALS}

For vessels of this size the optical enhancement provided by a pair of $4.3 \times$ Wide Angle Loupes (Designs for Vision) was deemed sufficient.

The EIEA was carried out in the 6 femoral arteries of 3 outbred Large White Landrace pigs with a mass of approximately $30 \mathrm{~kg}$. The animals were anaesthetised by means of an IV Sodium Pentothal induction and Fluothane inhalation maintenance.

Standard sterile precautions were followed. The groin areas were cleaned and shaved bilaterally and lazy-S skin incisions made, starting over the palpable femoral artery pulse and extending distally. The femoral sheath was exposed by means of blunt dissection and the femoral artery dissected free by means of hydro-dissection using a large polished-needle cannula. Small 
branches were occluded by means of bipolar diathermy as recommended by Caffee and Ward (30).

An approximating clamp pair of appropriate size was applied to the vessel and background material placed behind it. The vessel was divided between the clamps and the stumps rinsed with heparinised saline (30i.u/ml).

The vessel stumps were prepared as described in Chap.4 and the sutures placed by the modified method using double-armed $7 / 0$ sutures described in Chap.4.

The wounds were closed by interrupted sutures of 2/0 nylon. The animals were allowed to recover from their anaesthetics and transferred to their pens where they were fed and watered ad libitum.

After a period of 7-14 days they were again anaesthetised by the same method, the femoral arteries exposed and examined for patency and then removed for in vitro examination. The animals were not allowed to recover from this anaesthetic.

\section{RESULTS}

The anastomoses were simple and relatively quick to perform. In the first case, where the overlap had accidentally been made too short, it was necessary (to my chagrin) to place an extra suture.

The results are shown in Fig. 5.11.

The first vessel was also severely stenosed at evaluation. This vessel had taken longer than usual and required an additional suture, because the overlap length had been misjudged and had therefore been made too short. It was not completely occluded, but I classified it as such. 


\begin{tabular}{|c|c|c|c|c|}
\hline NO & $\begin{array}{c}\text { V.DIAM } \\
\text { (mm) }\end{array}$ & $\begin{array}{c}\text { OP. TIME } \\
\text { (min) }\end{array}$ & $\begin{array}{c}\text { FOLL. UP } \\
\text { (days) }\end{array}$ & $\begin{array}{c}\text { PATENCY } \\
\text { Y } / \mathrm{N}\end{array}$ \\
\hline \hline 1 & 2.5 & 11 & 14 & $\mathrm{~N}$ \\
2 & 2.5 & 7 & 14 & $\mathrm{Y}$ \\
3 & 3 & 5 & 7 & $\mathrm{Y}$ \\
4 & 3 & 6 & 7 & $\mathrm{Y}$ \\
5 & 2.5 & 4.5 & 9 & $\mathrm{Y}$ \\
6 & 2.5 & 5 & 9 & $83 \%$ \\
\hline \hline MEAN & $2.5+$ & 6.4 & 10 & $8 \%$ \\
\hline
\end{tabular}

FIGURE 5.11

EIEA in porcine femoral artery.

The other vessels were all patent and appeared to provide normal distal perfusion.

The vessels were opened longitudinally and examined under $40 \mathrm{X}$ magnification under the operating microscope.

Vessel No.1 was stenosed as a result of a fold in the FV. This probably occurred when the errant edge was pushed into the RV after it had slipped out. It appeared that the additional suture had actually fixed and maintained this fold.

All the other vessels, with the exception of No.4, had uniform glistening endothelial linings through which the scarring at the edge of the FV could vaguely be seen. All displayed very mild fusiform stenoses similar to those seen in the casts of the rat femoral artery (see Fig. 6.10).

In vessel No.4 the edge of the FV had lifted away from the wall of the RV for a very short distance in the area opposite to the deep attachment of the first suture loop. The appearance was similar to that of a tiny pocket (or of a miniature venous valve) and the entire pocket, including its outer adventitial 
surface, appeared to be covered by an endothelial layer with no adhesion of thrombus to any part of the inner surface of the anastomotic area.

\section{DISCUSSION}

The number of vessels in this study was too small to be predictive. The experience did, however, show me that it was possible to carry out the new sleeve anastomosis successfully in larger vessels by the modified method and that it may even be useful in the anastomosis of the troublesome hepatic artery in the pig.

I believe that further study of the anastomosis in larger vessels should be undertaken.

\section{SUMMARY}

A limited study of the new EIEA in porcine femoral arteries of $2.5 \mathrm{~mm}$ in diameter was performed. All but one of the 6 anastomoses were accomplished with ease, the first being troublesome due to inadequate overlap. All 5 of the vessels, which had been anastomosed easily, were patent at evaluation which ranged from 7-14 days post operation. Grossmicroscopy showed endothelial cover of all luminal surfaces and mild fusiform stenosis in the 5 patent vessels.

\section{PRIMATE VESSELS}

\section{AlMS}

Primates may be expected to have blood vessels which are very similar to those of their human cousins. Since the ultimate aim with any experimental surgical procedure is to apply the benefits 
to man, I thought it justifiable to carry out a limited number of EIEAs on Chacma baboons, which were due to be sacrificed in the course of other studies.

\section{METHODS AND MATERIALS}

Three adult Chacma, baboons which were to be sacrificed within a few days, were anaesthetised by the standard method of Sodium Pentothal induction, followed by Fluothane inhalation maintenance anaesthesia. Appropriate areas were shaved and cleaned and vessels exposed, clamped and prepared by standard surgical methods and the techniques described in Chap.4. The method for performing the EIEA was either the standard method or the doublearmed suture method described in Chap.4, chosen according to the size of the vessel.

The operating theatres were suited to macrovascular surgery and in this very limited study no provision was made for operating-microscope facilities in the theatre. All the EIEAs were therefore performed under the $4.3 \mathrm{X}$ magnification of my Loupe.

Vessels were examined and specimens obtained at the time at which the animal was due to be sacrificed according to the protocol of the other unrelated experiment.

\section{RESULTS}

There were no special difficulties in performing these anastomoses. The vessels appeared to me to be exactly similar to those I had encountered in man. In general their wall-thickness to lumen ratio was greater than in the other animal models, but this did not appear to have a major effect when the vessel had been dilated and treated with Xylocaine to prevent spasm.

The results of the EIEA in primate vessels are shown in Fig. 5.12. 


\begin{tabular}{|c|c|c|c|c|}
\hline ARTERY & $\begin{array}{c}\text { DIAM } \\
\text { (mm) }\end{array}$ & $\begin{array}{c}\text { TIME } \\
\text { (min) }\end{array}$ & $\begin{array}{c}\text { EVALU } \\
\text { (days) }\end{array}$ & $\begin{array}{c}\text { PATENCY } \\
Y / N\end{array}$ \\
\hline \hline FEMORAL & 4 & 6 & 14 & $Y$ \\
RADIAI & 1.5 & 7 & 9 & $Y$ \\
RADIAI & 1.5 & 5 & 11 & $Y$ \\
\hline
\end{tabular}

FIGURE 5.12

Results of EIEA in Chacma baboon arteries.

\section{DISCUSSION}

Here again the tabulated results were too few to be of any statistical value. It is, however, noteworthy that each of the three vessels was simply divided and then re-anastomosed and that such a situation would seldom occur in clinical practice. I have already mentioned that the wallthickness to lumen-diameter ratio is unfavourable to the sleeve anastomosis in small human arteries and these anastomoses (as listed in Fig. 5.12) had therefore been performed under unfavourable conditions. Indeed, the anastomoses performed in the radial arteries were performed in unfavourable vessels as compromisation of the prograde flow through those arteries could easily result in retrograde flow via the palmar arches. This would be tantamount to performing the sleeve anastomosis with a reversed overlap (i.e. inserting the RV into the FV)!

Considering these points, it was gratifying to observe definite patency and unobstructed flow through all 3 of the anastomoses. The success of the sleeve anastomosis under these adverse conditions leads me to agree with Meyer (133) that it should function well in clinical situations where the FV is slightly smaller than the RV. This could occur in some free-flap transfers and in the feeding vessel anastomosis in many interposition grafts.

\section{SUMMARY}

The sleeve anastomosis was used in direct reconstruction of three surgically divided arteries in the Chacma baboon. All the arteries in this very small sample were found to be patent at followup evaluation 9-14 days post operation. The vessels remained patent despite the facts that, in 
these relatively thick-walled arteries, the FV and RV were equal in diameter and that the two radial arteries might allow retrograde flow through the anastomoses.

\section{VENOUS ANASTOMOSES}

The veins of rats are extremely thin-walled and bear no resemblance to human blood vessels. They are, however, comparable to human lymphatic channels in the delicate nature of their structure. Lauritzen has evaluated the performance of the sleeve anastomosis in rat veins (109) and has found the results to be at least comparable to those obtained by ETEAs by other workers (78). Early failure of venous anastomoses is one of the problem areas in microvascular free-tissue transfer and replantation operations (27 [pp.25-28]) and indeed has been a problem in transplantation and replantation since the times of Carrel and Guthrie $(35,32)$. Special methods have been devised to supplement venous drainage until new venous channels have been established. Buncke advised removal of a section of the nail of a replanted digit and repeated "abrasive" cleaning of the nail bed with a heparin-soaked swab to allow oozing of excess blood and thus to prevent venous congestion (27 [p.28]). Other surgeons have restored the medical leech (Hirudo medicinalis) to its former glory for the same purpose (88).

\section{AlMS}

To evaluate the sleeve anastomosis in rat femoral veins on the basis of short-term patency and to identify any special problems associated with performing the new sleeve anastomosis in small veins.

\section{METHODS AND MATERIALS}

Twenty-four femoral veins in 12 adult male Sprague-Dawley rats were anastomosed by means of the new EIEA. 
The anaesthetic procedure, the preparation of the animals and the operative approach were exactly similar to those described for the femoral artery anastomoses. Dissection of the vein, separating it from the artery and the vascular sheath, was facilitated by hydro-dissection with the aid of the polished-needle cannula. Tributaries were occluded by micro-bipolar diathermy and then divided.

The standard method, with the modifications described in Chap.4, was employed for effecting anastomoses.

The animals received standard postoperative care and were evaluated under anaesthetic 5-18 days after the operation. At this time patency was assessed by Acland's "Stroking" test (1) and then, more rigorously, by observing the passage of clear fluid through the vessel when an intravenous injection of $\mathrm{KCl}$ was administered to terminate the experiment. Despite the fact that the first injection had killed the animal, a similar injection was performed into the opposite femoral vein to observe the passage of the clear fluid through the anastomosis. The walls of the veins were so thin that there was no difficulty in making the observations.

\section{RESULTS}

There were no difficulties with the operative technique. All the anastomoses were performed expediently and there was no reason to suspect that any one of them might fail.

All the animals survived and were available for follow-up evaluation. There were no discrepancies between the two patency tests.

The results are shown in Fig. $\mathbf{5 . 1 3}$ 


\begin{tabular}{|c|c|c|c|c|}
\hline$\underset{\#}{\text { ANIMAL }}$ & $\begin{array}{l}\text { SIDE } \\
\mathrm{L} / \mathrm{R}\end{array}$ & $\begin{array}{l}\text { DIAM } \\
(\mathrm{mm})\end{array}$ & $\begin{array}{l}\text { ASSESS. } \\
\text { (days) }\end{array}$ & $\begin{array}{l}\text { PATENT } \\
\mathrm{Y} / \mathrm{N}\end{array}$ \\
\hline \multirow[t]{2}{*}{1} & $\mathbf{R}$ & 1.6 & 7 & $Y$ \\
\hline & L & 1.5 & 7 & $Y$ \\
\hline \multirow[t]{2}{*}{2} & $\mathbf{R}$ & 1.2 & 7 & $Y$ \\
\hline & I & 1.2 & 7 & N \\
\hline \multirow[t]{2}{*}{3} & $\mathbf{R}$ & 1.3 & 9 & $Y$ \\
\hline & L & 1.3 & 9 & $Y$ \\
\hline \multirow[t]{2}{*}{4} & $\mathbf{R}$ & 1.2 & 7 & $Y$ \\
\hline & L & 1.3 & 7 & $Y$ \\
\hline \multirow[t]{2}{*}{5} & $\mathbf{R}$ & 1.3 & 5 & $\mathbf{N}$ \\
\hline & L & 1.3 & 5 & $Y$ \\
\hline \multirow[t]{2}{*}{6} & $\mathbf{R}$ & 1.4 & 11 & $\mathbf{N}$ \\
\hline & L & 1.4 & 11 & $Y$ \\
\hline \multirow[t]{2}{*}{7} & $\mathbf{R}$ & 1.2 & 16 & $\mathrm{Y}$ \\
\hline & I & 1.2 & 16 & $Y$ \\
\hline \multirow[t]{2}{*}{8} & $\mathrm{R}$ & 1.2 & 18 & $\mathbf{N}$ \\
\hline & L & 1.2 & 18 & $Y$ \\
\hline \multirow[t]{2}{*}{9} & $\mathbf{R}$ & 1.3 & 12 & $Y$ \\
\hline & I & 1.3 & 12 & $\mathbf{Y}$ \\
\hline \multirow[t]{2}{*}{10} & $\mathbf{R}$ & 1.6 & 8 & $Y$ \\
\hline & L & 1.6 & 8 & $Y$ \\
\hline \multirow[t]{2}{*}{11} & $\mathbf{R}$ & 1.4 & 9 & $Y$ \\
\hline & L & 1.3 & 9 & $Y$ \\
\hline \multirow[t]{2}{*}{12} & $\overline{\mathrm{R}}$ & 1.3 & 6 & $\bar{Y}$ \\
\hline & $\mathrm{L}$ & 1.2 & 6 & $Y$ \\
\hline $\begin{array}{l}\text { TOTAL } \\
\text { MEAN }\end{array}$ & 24 & $\begin{array}{r}31.8 \\
1.32\end{array}$ & $\begin{array}{l}230 \\
9.6\end{array}$ & $\begin{array}{l}4 \\
16.6 \%\end{array}$ \\
\hline
\end{tabular}

FIGURE 5.13

The EIEA in rat femoral veins.

Only one of the four occluded vessels had any hint of an apparent cause for failure. This vessel $(6 \mathrm{R})$ was surrounded by inflammatory exudate and the surrounding tissues were inflamed, thus indicating infection. The operations had been performed under clean, but unsterile conditions.

The mean diameter of the occluded vessels $(1.28 \mathrm{~mm})$ was slightly smaller than the mean for the sample $(1.32 \mathrm{~mm})$, but the difference of the means was not statistically significant. $(t=0.908$ therefore $p>0.05$ ) 


\section{DISCUSSION}

The patency rate of $20 / 24(83.3 \%)$ in these veins was disappointing, but it was not significantly different from the 19/21 (90.4\%) rate obtained by Lauritzen and Hansson (109) with the sleeve anastomosis $\left(X^{2}=0.5706-p>0.05\right)$. When compared to the rate of $40 / 50(80.0 \%)$ obtained by Hayhurst and $O$ 'Brien (78), there was also no significant difference $\left(X^{2}=0.1189-p>0.05\right)$. The analysis of patency rate at different follow-up times, which indicated to Hayhurst and O'Brien that there was a greater incidence of venous occlusion in the second postoperative week, was not corroborated by this study, in which we found that there was no significant difference between venous patency for veins examined at different intervals $\left(X^{2}=.593\right.$; $d f=2-p>0.05$ ).

\section{SUMMARY}

The new EIEA was used to reconstitute 24 femoral veins in rats. The patency rate of $83 \%$ was not significantly different from that achieved in other studies. It appears that the new EIEA may be safely used in the anastomosis of small veins.

\section{UNUSUAL VESSELS (RAT PORTAL V.)}

The rat portal vein has a helical lumen (Fig. 5.14). The muscular layers of the vessel wall display slow, rhythmic, contractile movements, which do not occur in other vessels (20).

Kamada and Calne (97) have routinely successfully reconstituted the rat portal vein by means of a cuff technique, based on the method of Payr $(154,81)$, in their liver transplantation programme. Their reason for using this method is mainly to save time. Lee et al (119) have used a continuous suture ETEA for the same purpose. 
The sleeve anastomosis, if practicable in this strange vessel, would obviate the use of bulky foreign material required by the Kamada cuff technique while also saving time in comparison with other methods.

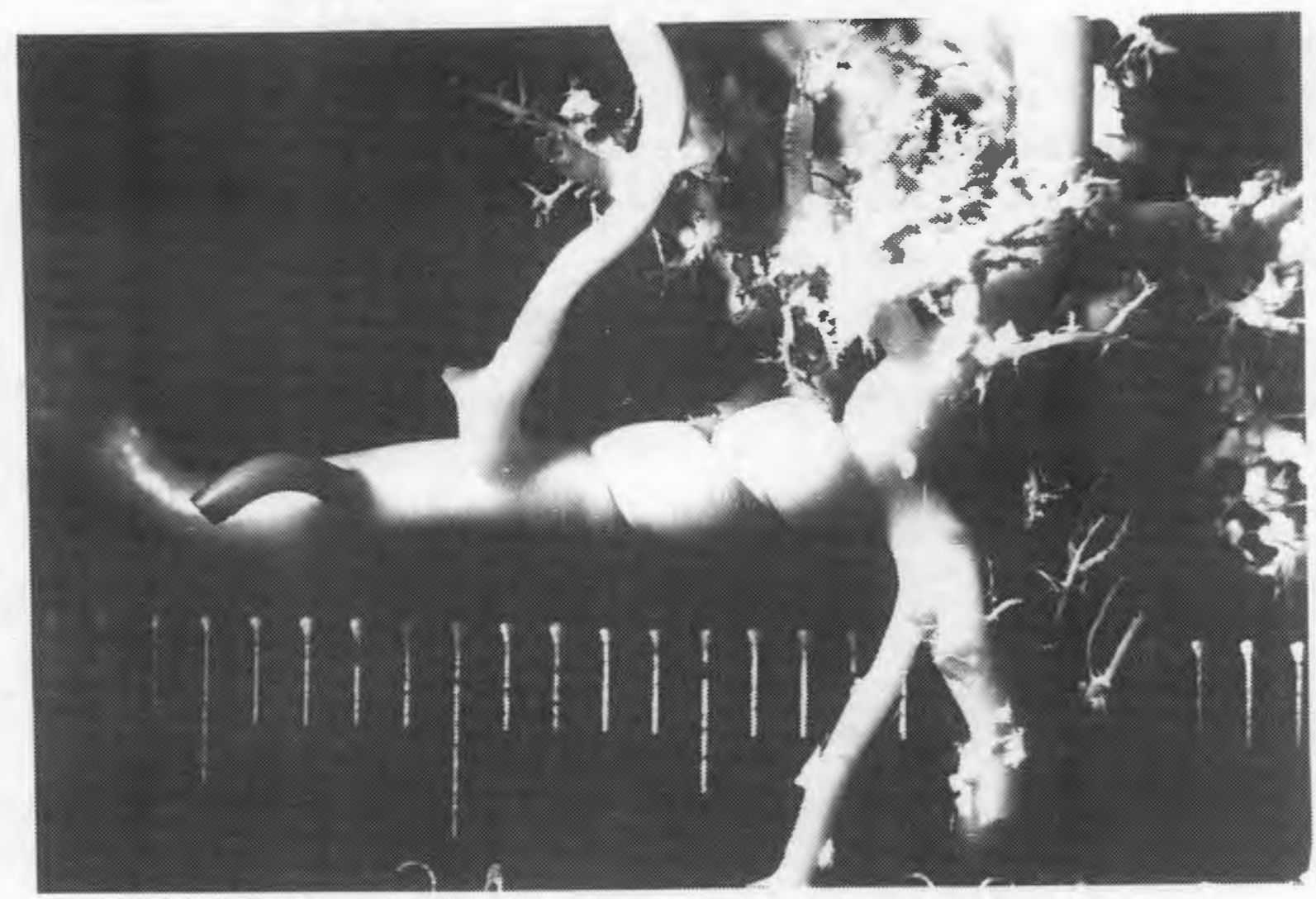

FIGURE 5.14

The helical shape of the rat's portal vein.

\section{AlMS}

To ascertain if the sleeve anastomosis could be employed effectively in the portal vein of the rat so that it might be used in irepatic transplantation research programmes.

\section{METHODS AND MATERIALS}

Eight adult male Long-Evans rats $(300 \mathrm{~g}-400 \mathrm{~g})$ were anaesthetised by ether inhalation and positioned supine on an operating table. The abdominal hair was shaved off from the 
xiphisternum to the pubis and a midline laparotomy was performed under clean but unsterile conditions.

The abdominal wound was held open by elasticised retractors and surrounded by gauze swabs moistened with normal saline solution. The bulk of the bowel was reflected to the animal's left side, packed out of the peritoneal cavity onto a moist swab and covered by another moist swab. The portal vein was then dissected free, as in the preparation for hepatic transplantation (119) with division of the pyloric tributary. The vessel was then divided between clamps and reconstituted by means of the standard new EIEA.

The peritoneal cavity was rinsed with a $\mathrm{N} / \mathrm{saline}$ solution, the bowels were replaced and the abdominal wall and peritoneum closed in one layer by means of a running suture of $4 / 0$ chromic catgut.

Four of the operations were performed by a colleague and I was responsible for the remaining four.

Approximately 6 weeks later the animals were anaesthetised by means of Ketamine/Acepromazine and the anastomoses evaluated by means of a corrosion cast via the splenic vein.

\section{RESULTS}

All the animals survived and remained apparently well. They were eating and drinking normally from the first or second postoperative day until the time of the evaluation. No attempt was made to assess portal vein pressure or liver function.

The results are tabulated in Fig. 5.15. 


\begin{tabular}{|c|c|c|c|c|c||}
\hline NO. & SURGN & $\begin{array}{c}\text { DIAM } \\
\text { (mm) }\end{array}$ & $\begin{array}{c}\text { TIME } \\
\text { (min) }\end{array}$ & $\begin{array}{c}\text { EVALU. } \\
\text { (days) }\end{array}$ & $\begin{array}{c}\text { PATENCY } \\
\text { Y/N }\end{array}$ \\
\hline \hline 1 & FD & 1.2 & 6 & 40 & $Y$ \\
2 & FD & 1.4 & 7 & 42 & $\mathrm{Y}$ \\
3 & GE & 1.2 & 6 & 40 & $Y$ \\
4 & FD & 1.3 & 6 & 44 & $Y$ \\
5 & GE & 1.5 & 7 & 46 & $Y$ \\
6 & FD & 1.4 & 7 & 42 & $Y$ \\
7 & GE & 1.6 & 6 & 44 & $Y$ \\
8 & GE & 1.4 & 9 & 49 & $Y$ \\
\hline
\end{tabular}

FIGURE 5.15

Results - the EIEA in the portal vein of the rat.

Photomicrographs of some of the corrosion casts are shown in Fig. 5.16. Patency of the portal vein was confirmed by the corrosion casts in all cases. However, in some cases the topography of the vascular lumen had been severely disturbed and it was noteworthy that in those cases the animals had also gained an abnormal amount of weight.

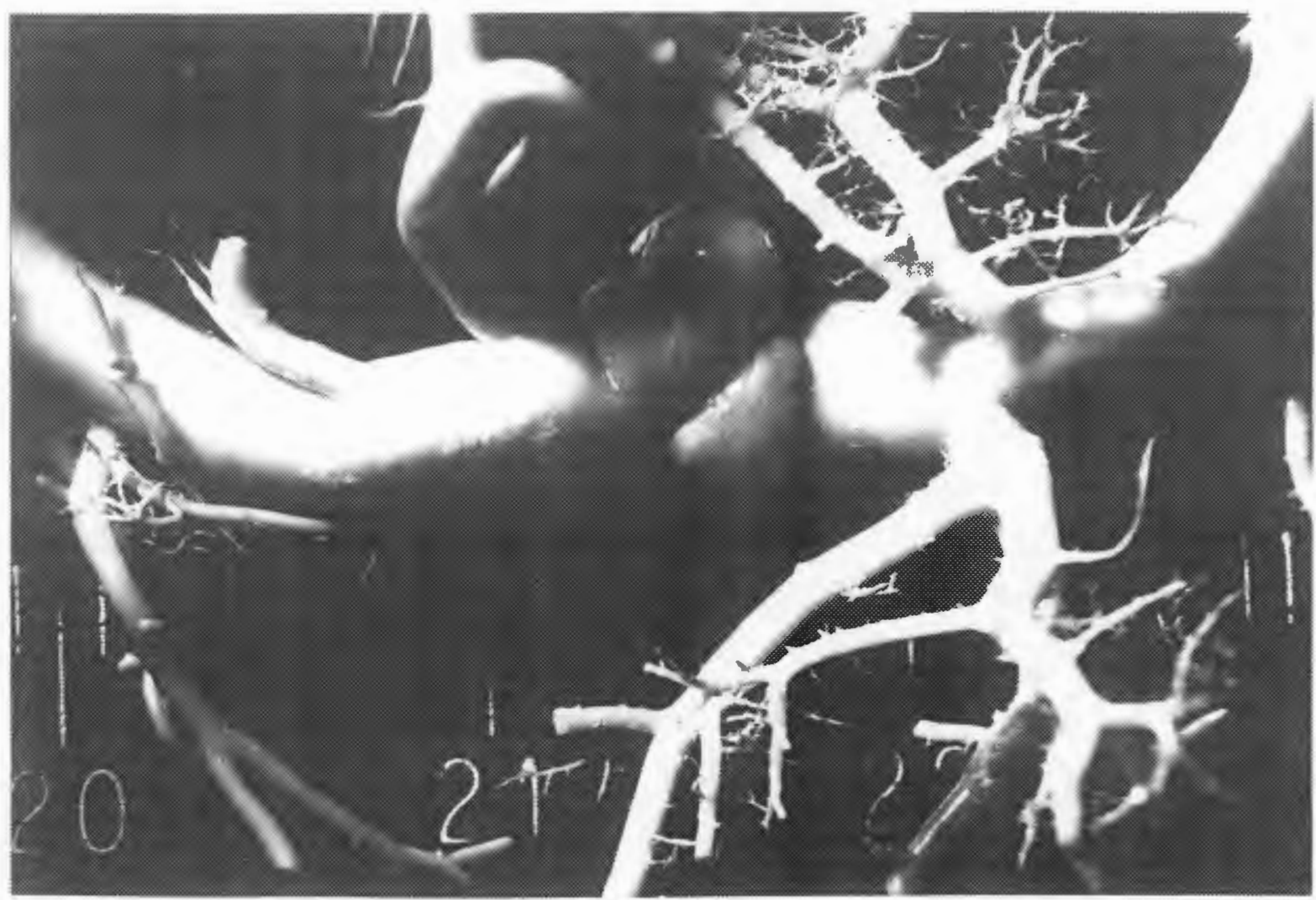

FIGURE 5.16

Corrosion cast of the portal vein of the rat following sleeve anastomosis of the vessel. 


\section{DISCUSSION}

The fact that all the portal veins remained patent, was at first surprising in the light of our previous experience with venous anastomoses by means of the EIEA and the special characteristics of the portal vein. However, analysis by the $X^{2}$ test showed that the result obtained with this small sample was not significantly different from that obtained in femoral veins $\left(X^{2}=1.524 ; d f=1-P=0.25\right)$.

The portal vein corrosion casts did not reveal any stenosis of the vessel at the anastomotic site, but there was marked distortion of the helical pattern in 3 cases (see Fig. 5.16). There were no clues as to the cause of the distortion or the relationship between the distortion and the abnormally increased growth of the rats. I have embarked upon a separate series of studies to investigate these phenomena.

This study indicated to us that the new EIEA could be successfully used with relatively short operating time to reconstitute the portal vein of the rat in such a way that it remained patent. We are, however, neither certain as to how this method compares with others in its alteration of the topography of the vascular lumen, nor do we know what the physiological effects of such topographical alterations may be.

\section{SUMMARY}

The new EIEA was used in 8 rat portal veins. The procedures took 6-7 minutes (vessel division to final clamp release). All the animals survived until evaluation of the anastomoses was performed 6 weeks later. All the vessels were patent at evaluation, but there was severe disturbance of the topography of the helical vascular lumen in 3 cases. In these 3 cases the animals had gained weight at an abnormal rate. The relationship between disturbance of the portal vein architecture and weight gain is still obscure. 


\section{CHAPTER VI \\ COMPARATIVE STUDIES}

Nothing is good or bad but by comparison.

Thomas Fuller M.D. (1732)

\section{EIEA VS ETEA IN RAT FEMORAL ARTERY}

\section{AlMS}

In this group of studies I wished to compare different parameters in the new sleeve anastomosis with their counterparts in a standard ETEA. In order to do this, I had to ensure that I had a comparative model, in which biasing factors had as far as possible been eliminated. Rat femoral arteries seemed to provide a good paired system of vessels in which pairs of differing anastomoses could be compared. If the two different anastomoses were performed at one sitting, they would be allowed to heal under exactly similar physiological conditions. However, even in such a model it may be possible for biasing factors to occur.

Some of the factors which I thought might create bias were:

1. The order in which the anastomoses were to be performed. The surgeon might be tiring by the time he starts the second anastomosis, or on the other hand he might become more adept after a shaky start. 
2. The side on which an anastomosis was to be performed. If the rat is always lying supine with its tail towards the surgeon, it might for example be easier to operate on the left femoral artery than on the right.

3. The standard of vessel preparation. The experimental surgeon, who subconsciously favours one of the anastomoses, may perform a more careful dissection of the vessel on the side where his favourite anastomosis is to be performed.

I devised a protocol which would eliminate as many as possible of the biasing factors which might have been lurking in my own subconscious mind.

\section{METHODS AND MATERIALS}

\section{PROTOCOL}

Male Sprague-Dawley rats $(300-400 \mathrm{~g})$ were used in the study. Each animal was anaesthetised with an intraperitoneal injection of a mixture of Ketamine $10 \mathrm{mg} / 100 \mathrm{~g}$ body weight and Acepromazine $5 \mathrm{mg} / 100 \mathrm{~g}$ body weight. The lower abdomen and groin areas were shaved by means of an electric clipper and loose hairs were removed by means of suction airflow. The animal was placed on its back on an operating board and position was maintained by means of loose restraints to the limbs and tail.

I performed all the operations. A $3-4 \mathrm{~cm}$ midline abdominal skin incision was made and, with some reflection of the fat pads and adjustment of retractors, the groin areas were exposed one at a time. The femoral artery was dissected clear, mainly by hydrodissection with the polished needle caninula, from the inguinal ligament to the superficial epigastric branch. Deep muscular branches of the femoral artery were occluded by bipolar coagulation and then divided. Background material was placed under the vessel and the area was irrigated with Ringer's Lactate solution with 1:100 Povidone lodine and covered with a moistened gauze sponge, before proceeding with the dissection of the opposite side in an exactly similar manner. 
When both femoral arteries had been dissected and isolated by background material, a number from 1 to 30 was randomly drawn from a box. Odd numbers drawn assigned the new sleeve technique to the right side and the ETEA to the left and viceversa for even numbers. No matter which number was drawn, the right side was always operated on first. By these methods it was hoped to eliminate all bias with regard to side, sequence or quality of dissection for either anastomosis and thus to obtain a set of anastomoses which could be compared on the basis of their type alone. For each anastomosis the approximating clamp was placed with its proximal jaw very near to the divided deep branch. The vessel was therefore divided between the deep branch and the superficial epigastric artery (Fig. 6.1). No removal of adventitia was performed before division of the vessel.

For every anastomosis a stopwatch was started at the time of vessel division and it was stopped upon the final removal of the clamps. All irrigating solutions were the same: Heparin $2000 \mathrm{u} / 100 \mathrm{ml} \mathrm{N}$. Saline, Ringer's Lactate with 1:100 Povidone lodine and Xylocaine $2 \%$ for vessel dilation. The same suture material $10 / 0$ Nylon on a DRM-4 needle by Xomed was used for all anastomoses.

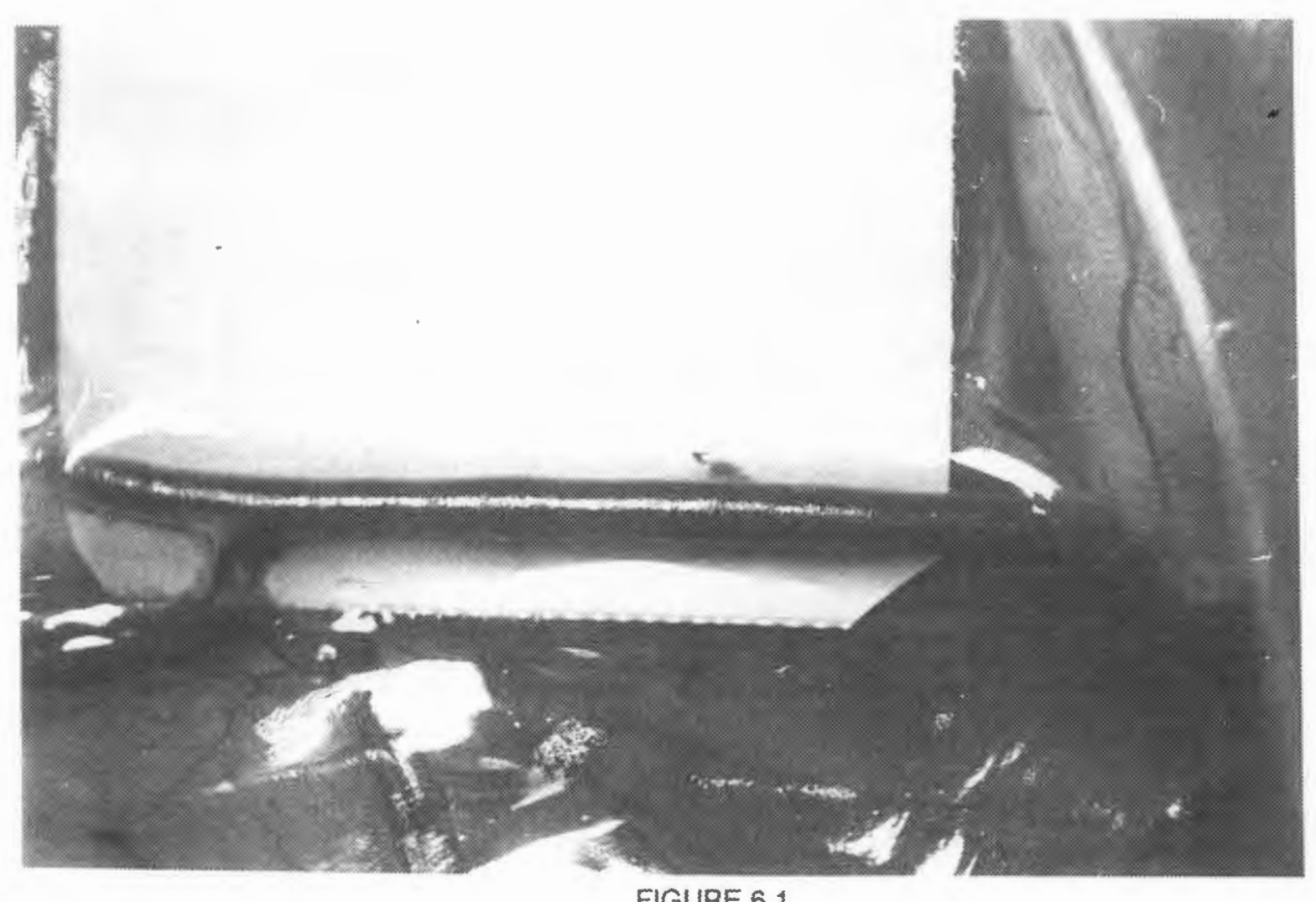

FIGURE 6.1

The vessel was civided between the deep branch and the superficial epigastric artery 


\section{Chapter 6 - Comparative Studies}

The ETEA technique used was the "posterior wall first", technique described by Harris, Finseth \& Buncke (76) and 8 to 10 sutures were used per anastomosis (Fig. 6.2).

When both anastomoses had been completed, the abdominal wound was checked for haemostasis, washed out with the Ringer's Lactate / Povidone lodine solution and sutured with a continuous 4/0 Nylon suture. The rat, while still anaesthetised, was marked by means of an alphanumeric identifying code tattooed into the left ear. Each animal was given a $5 \mathrm{ml}$ subcutaneous injection of Ringer's Lactate solution to compensate for intra-operative fluid losses, after which it was placed near a heating pad until fully recovered from the anaesthetic. The identifying code of each rat, together with the date, type of anastomosis on each side and time taken for each was recorded. Animals were selected for evaluation by random drawing of identity slips.

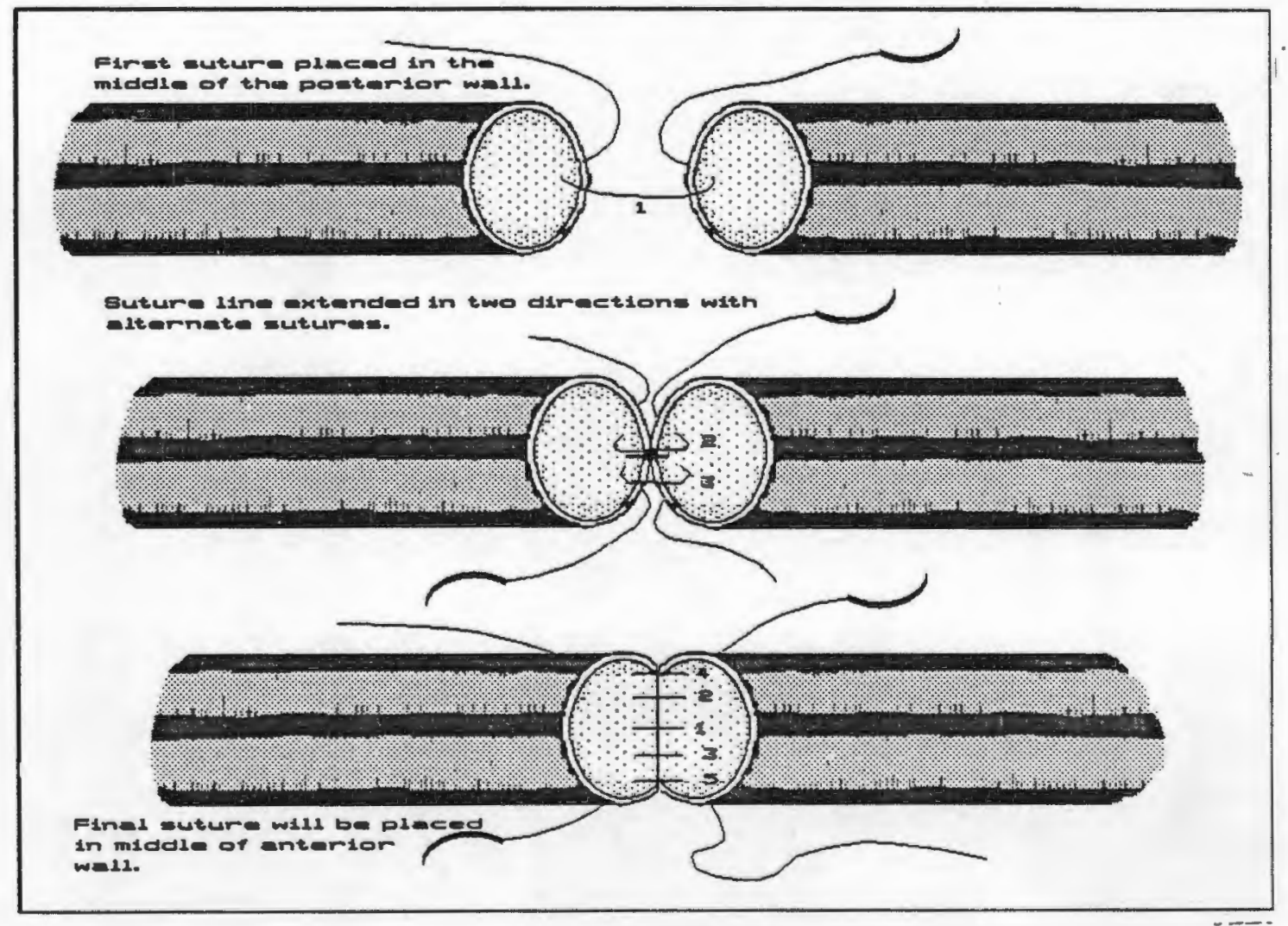

FIGURE 6.2

The "posterior wall first" technique described by Harris, Finseth \& Buncke (76) 


\section{EVALUATION METHODS}

In each animal which had been prepared in accordance with the protocol, there was a pair of anastomoses which could now be compared with one another on the basis of chosen parameters.

\section{Time Taken}

As mentioned in the protocol, the time taken for each anastomosis from the time of vessel division to the final release of the clamps, was recorded by means of a stopwatch. This electronic stopwatch had a "lap-time" facility, which meant that it could be re-stopped if the need for re-application of clamps arose.

The elapsed times were converted from minute/second to decimal format for the purpose of statistical analysis.

\section{Patency}

The most important characteristic of any form of anastomosis is undoubtedly its potential for remaining patent. All anastomoses were evaluated for patency in at least one of the following ways:

1. Direct observation and patency testing $(1,78)$ after anatomical dissection.

2. Observation of flow through the anastomosis by the method of Lauritzen and Bagge (108).

3. Observing the morphology of vascular corrosion casts of vessels in which anastomoses had been made. 
Chapter 6 - Comparative Studies

\section{Morphology}

The shape of the lumen of the vessel in the region of the anastomosis was studied by means of the exact reproductions of the luminal shapes, which were obtained by means of resin corrosion casts, as described in Appendix B.

Each pair of casts was photographed, as described in Appendix B and measurements were then obtained from the photographs.

The system by which measurements were obtained from the photographs is demonstrated in Fig. 6.3.

Measurements were taken at the anastomotic site and at a distance of $2 \mathrm{~mm}$ on either side of the site. In the sleeve anastomosis the narrowest area that could be found, was taken as the "anastomotic site". Each vessel was photographed twice, i.e. once in each of its widest and narrowest profiles. The two diameters were then scaled according to the width of the standard rod and the results used to calculate:

1. the mean diameter at each of the 3 measuring points; and

2. the cross sectional area of the vessel at each measuring point, using the formula for the area of an ellipse: $A=P i / 4 \times 1 \times s$. 


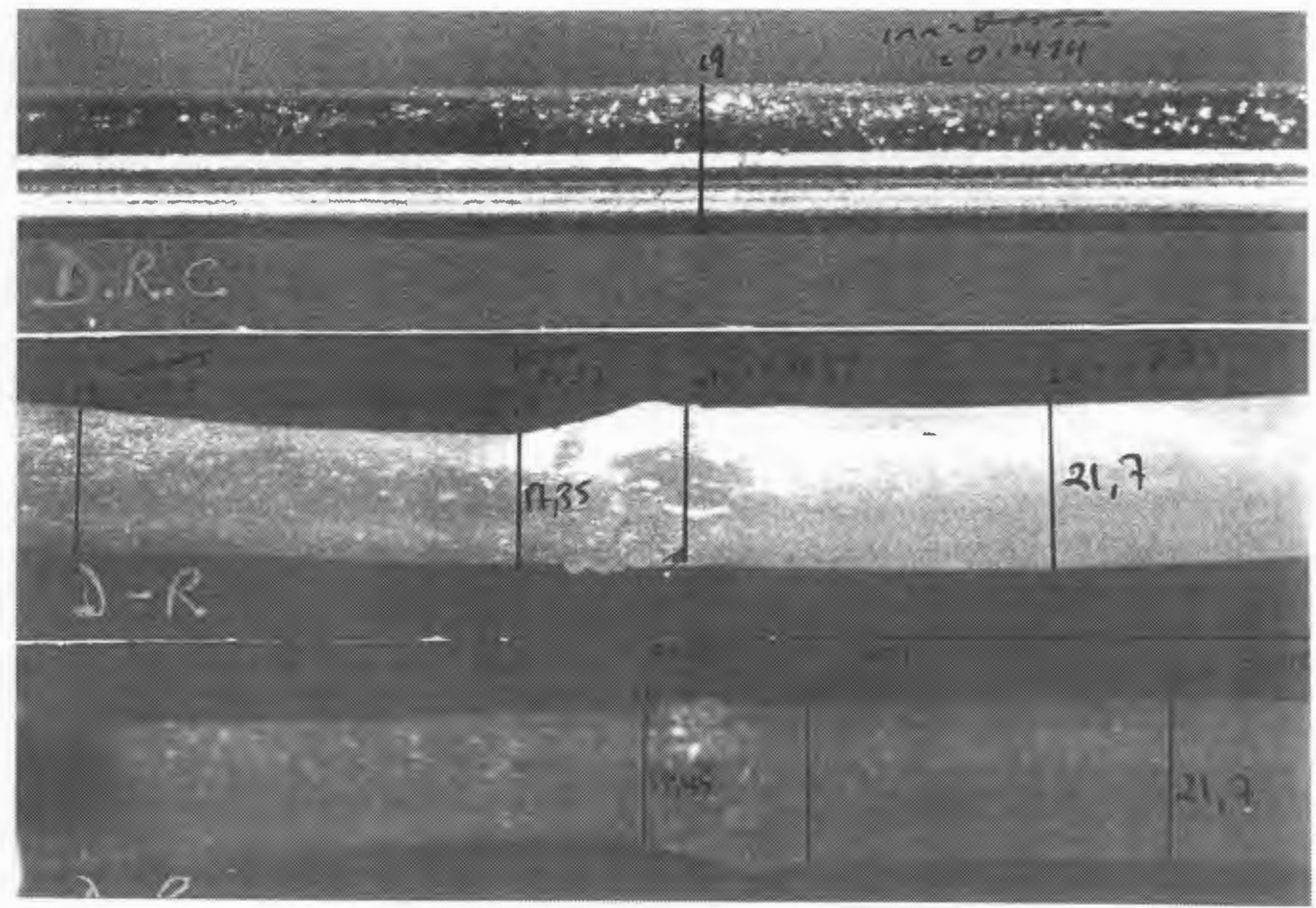

FIGURE 6.3

The system by which measurements were obtained from the photographs of corrosion casts.

\section{Flow}

The establishing of flow rates in very small vessels is practicable with the aid of sophisticated measuring devices such as Pulsed Ultrasonic Doppler Velocity Meters (PUDVM) (19) and Laser Dopplers (80). The expense incurred in obtaining such equipment is justifiable in clinical practice but not in our experimental situation. Since I only wanted to compare flow through two conduits subjected to identical forces, I considered the method of Lauritzen and Bagge (108), with slight modification, to be adequate for the purpose. The modifications consisted of:

1. introducing exactly similar "collecting" cannulae into the RV's distal to each of the anastomoses; and 
2. measuring the flow through both vessels simultaneously, by using an elevated IVinfusion bottle with a wide-bored $I \mathrm{~V}$-administration set as a pressure head.

3. allowing outflow through both vessels simultaneously, so that they would be exposed to exactly similar driving pressures throughout the measuring period.

4. not limiting the volume of fluid to $50 \mathrm{ml}$, as they had done. I allowed free flow through the vessels for a period of 4 minutes and then measured the collected fluid volume. This was done by weighing the container and the fluid on an electronic scale and subtracting the mass of the container. The volume of the fluid was taken as $1 \mathrm{ml}$ per gramme mass.

Flow rates were also obtained from control animals which were matched for body mass to some of the test animals.

\section{Tensile strength}

In theory, the tensile strength of a standard ETEA depends on the collective strength of the sutures holding the anastomosis. The tensile strength of each suture in turn depends on the strength of the suture material and the strength of the tissue in which the suture has been placed. There are, conceivably, situations in which the tensile strength of the anastomosis is not equal to the sum of individual suture strengths. Such situations may occur when sutures have not been placed with even spacing and equal tension. Application of an axial tensile stress to such a vessel will place greater stress on the tighter sutures which, called upon to counter the concentrated load, will break down early, thus transferring a greater load to the remaining sutures. This will result in their breakdown at lower loads than might have been expected if the stress had been evenly shared by all the sutures. In the living blood vessel the adhesive strength of the fibrin clot which forms between and around the vessel ends may also contribute to the tensile strength of the anastomosis. Later in the healing process the tensile strength of the anastomosis is dependent mostly upon the strength of the connective tissue which has taken part in the healing process.

In the EIEA the tensile strength is theoretically due largely to the frictional forces between the overlapping walls and those forces are only meaningfully present when there is pressure within the vessel. 
Bearing these theories in mind, I did not consider it either accurate or meaningful to apply either the spring-balance test used by Colen et al (46) or the more sophisticated material-testingmachine test as used by Lauritzen et al (108) to the EIEA. In order to obtain meaningful results, I reasoned that the tensile strength of the EIEA should be tested while the vessel was pressurised from within.

Fine-bored plastic intravenous catheters (Intracath ${ }^{T M} 23$ Gauge) were given slightly flared ends by means of heat treatment. They were then inserted into the cut ends of a freshly dissected segment of vessel and fixed by means of a ligature tied over the vessel and behind the flared end of the tube (see Fig. 6.4).

Each of two lengths of plastic tubing, which fitted snugly over the catheters, had a hole made in one side. They were then slipped over the catheters and glued to them. A length of the outer tube remained attached to each of the collars (Fig. 6.5).

These pieces of tubing were held in the metering-clamps of a tensiometer. In this way measurable tensile stress could be applied to the vessel segment and its anastomosis via the outer tube and the intravenous catheters, while the vessel could simultaneously be subjected to normal intraluminal pressure via the catheter. Pressure of $120 \mathrm{~mm} \mathrm{Hg}$ in the vessel was provided by allowing a balanced solution of electrolytes (Plasmalyte $B$ ) to run slowly through it. The outflow was controlled to maintain an open-ended column of water on the inflow side at a height of $163 \mathrm{~cm}(13.6 \times 120 \mathrm{~mm})$ above the vessel. The "plumbing" was fashioned from a Central Venous Pressure Manometer set and a standard IV fluid administration set. Fig. 6.6 shows the entire apparatus. 


\section{Chapter 6- Comparative Studies}

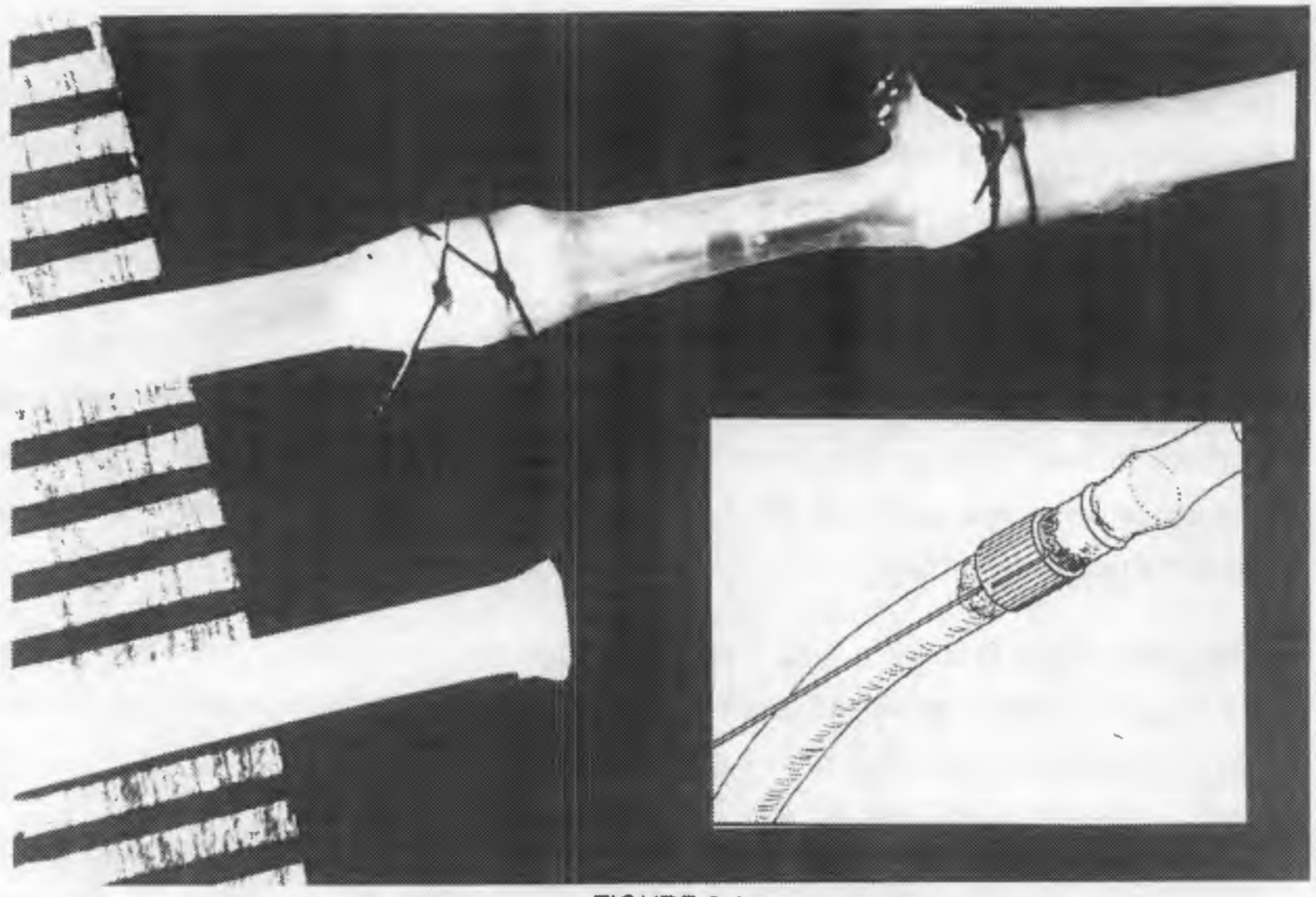

FIGURE 6.4

Preparation of the vessel for tensile-strength measurement.

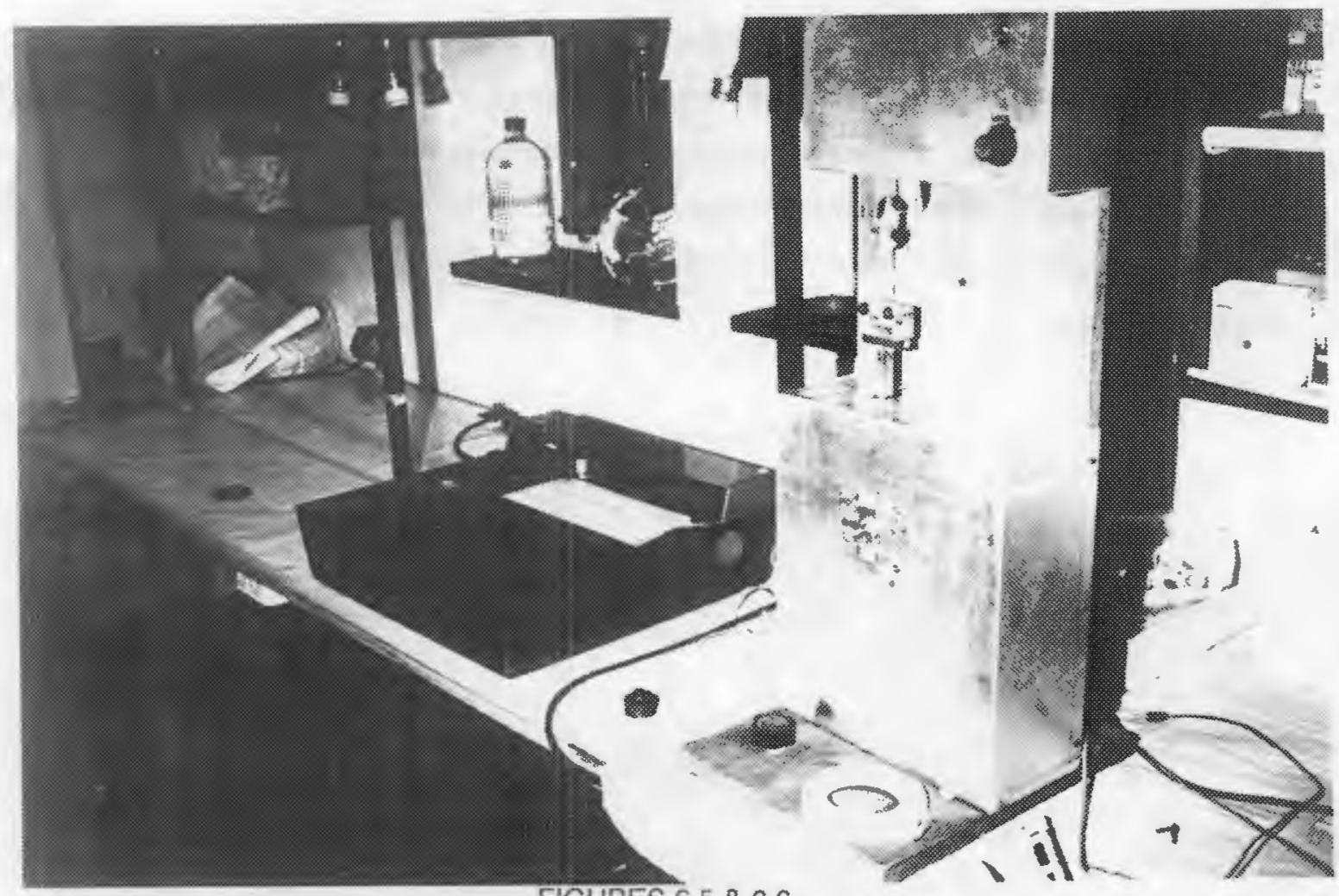

FIGURES $6.5 \& 6.6$

The apparatus used for measurement of tensile strength of the vessels. 


\section{RESULTS}

\section{TIME TAKEN}

Ninety-seven pairs of anastomoses were timed, but in the case of the sleeve anastomosis the first 8 operating times had to be discarded to make the sample homogeneous $\left(F=1.52 ;{ }^{d f} F_{W}=78\right.$; $\left.d f_{B}=10-P>0.05\right)$.

The 97 ETEA operating time data were not homogeneous ( $\left.F=2.92 ; \mathrm{df}_{W}=85 ; \mathrm{df}_{B}=11-P<0.01\right)$, but they were very close to homogeneity if the first 8 were discarded $\left(F=2.10 ; d_{W}=78 ; d_{B}=10\right.$ $-P$ 0.05). I have therefore chosen to separate the large homogeneous group of 89 pairs and the small group consisting of the first 8 pairs of anastomoses which I had performed.

The distributions of operating time data for the two different types of anastomoses are shown in Fig. 6.7.

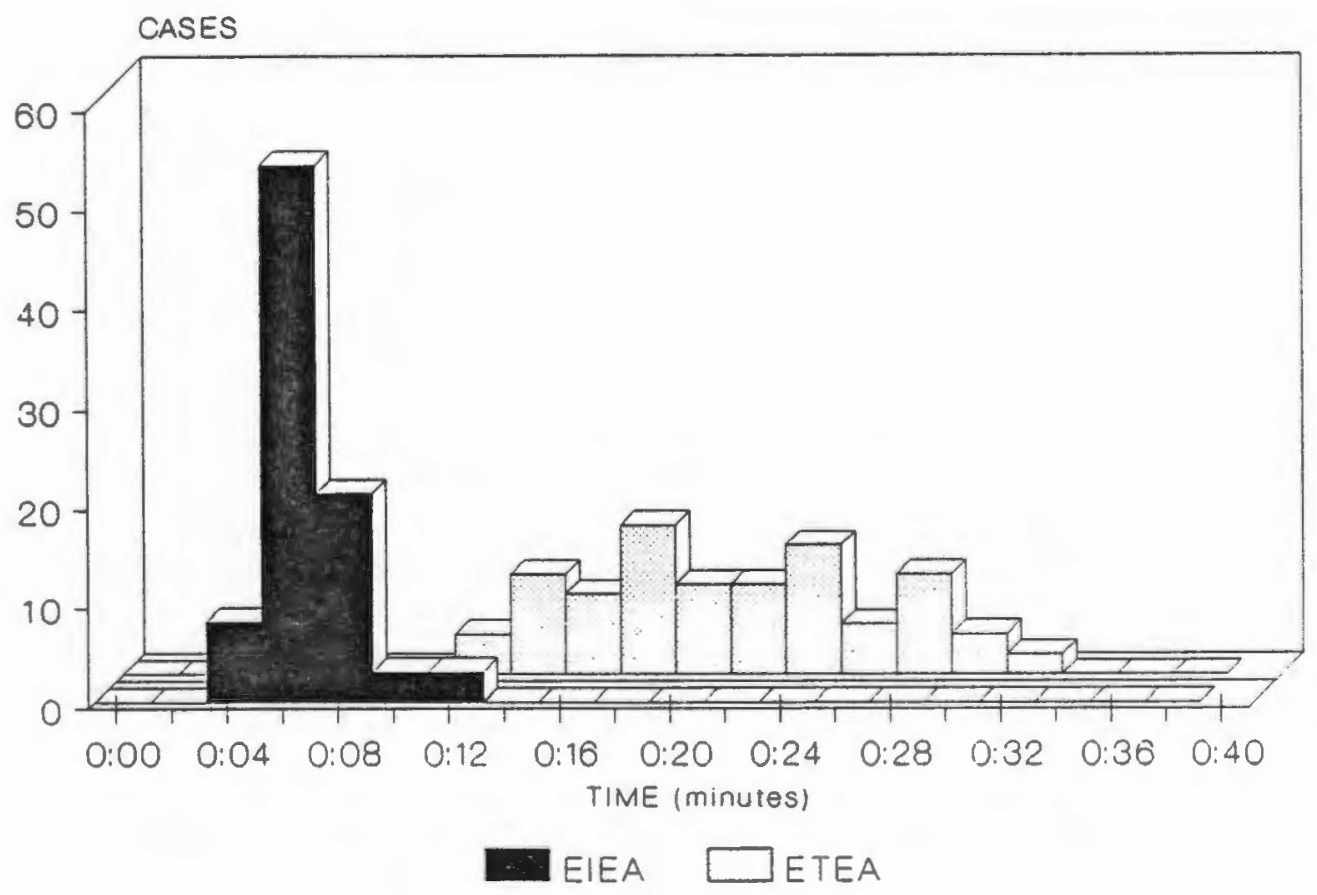

FIGURE 6.7

The distributions of operating-time data for the two different types of anastomoses 
As might be deduced from the graph, the difference in operating time between these groups was significant $(S E D=0.3124 ; D=13.364$; thus $D>\{3 \times$ SED $\}$ and therefore $P<0.01)$. The statistical analysis of operating time for each of the two anastomoses is tabulated in Fig. 6.8.

\begin{tabular}{|c|c|c|c|c|c|}
\hline $\begin{array}{l}\text { ANAST } \\
n=89\end{array}$ & $\begin{array}{l}\text { MAXIMUM } \\
\text { min }\end{array}$ & $\begin{array}{l}\text { MINIMUM } \\
\text { min }\end{array}$ & $\begin{array}{c}\text { MEAN } \\
\text { min.sec }\end{array}$ & $\begin{array}{l}\text { STAN. DEV } \\
\text { min.sec }\end{array}$ & $\begin{array}{l}\text { VARIANÇE } \\
\text { (min) }^{2}\end{array}$ \\
\hline $\begin{array}{l}\text { EIEA } \\
\text { ETEA }\end{array}$ & $\begin{array}{l}13.50 \\
28.17\end{array}$ & $\begin{array}{r}5.45 \\
14.38\end{array}$ & $\begin{array}{r}7.36 \\
20.57\end{array}$ & $\begin{array}{l}1.36 \\
3.19\end{array}$ & $\begin{array}{r}2.59 \\
11.02\end{array}$ \\
\hline
\end{tabular}

FIGURE 6.8

Analysis of comparative operating-time data for EIEA vs. ETEA.

There was a high positive correlation between the operating times of the matched pairs (?=0.462; $d f=89-P<0.001)$, hence the correlation-corrected test used for the analysis of the SED above.

\section{PATENCY}

Four animals were lost to follow-up for patency evaluation. The results in the remaining 93 pairs are tabulated in Fig. 6.9.

\begin{tabular}{|l|c|c|c|c|}
\hline ANAST & TOTAL & PATENT & OCCLUDED & PATENCY \\
\hline \hline EIEA & 93 & 92 & 1 & $98.9 \%$ \\
ETEA & 93 & 88 & 5 & $94.6 \%$ \\
\hline
\end{tabular}

FIGURE 6.9

Comparative patency rate of EIEA vs. ETEA

The patency rates of the two anastomoses were not significantly different $\left[X^{2}=2.75 ; d f=1\right.$ $P>0.05]$. 


\section{MORPHOLOGY}

Corrosion casts were made in 42 pairs of blood vessels. There were no casting failures. The general subjective impressions gained from the casts were as follows:

\section{EIEA (Fig. 6.10 A)}

1. The EIEAs usually displayed a slight fusiform stenosis corresponding to the overlap area.

2. The inner surface of the vessel was very smooth and gave the impression that there would be little, if any, disturbance of the laminar-flow pattern.

3. There was a slight dilation of the vessel distal to the anastomosis.

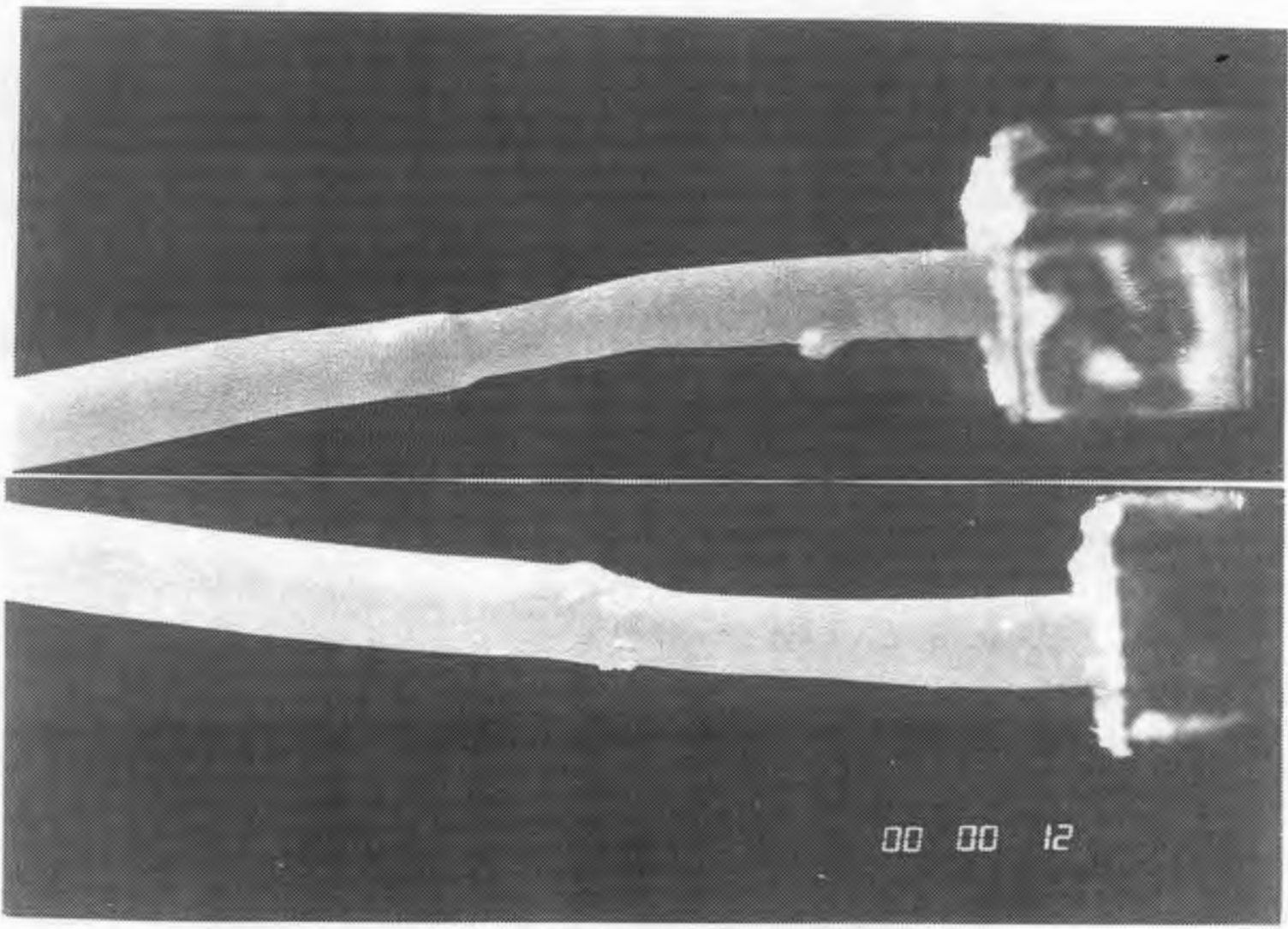

FIGURE 6.10

Comparative corrosion casts made in the femoral arteries of a rat which had an EIEA in one limb [A] and an ETEA in the opposite limb [B]; note the smooth stenosis of the EIEA and the irregularities of the luminal surface of the ETEA. 
Chapter 6- Comparative Studies

4. There were no aneurysmal dilatations associated with any of the sleeve anastomoses.

\section{ETEA (Fig. 6.10 b)}

1. The vessels were not physically stenosed at the anastomotic site.

2. The inner surface of the vessel displayed numerous small irregularities in the vicinity of the anastomosis. These irregularities were probably caused by sutures and gave the impression that they might interfere with laminar flow and thus cause turbulence.

3. There was slight dilation of the vessel distal to the anastomosis.

4. Aneurysms were present in 12 of the 42 ETEAs (28.6\% incidence). In 3 cases sutures were found imbedded in the resin on the outer surface of an aneurysm, thus indicating that these were true aneurysms.

Figure 6.11 shows a pair of vessels in which the typical mild fusiform stenosis of the sleeve anastomosis and a large aneurysm of the ETEA vessel can be seen. 


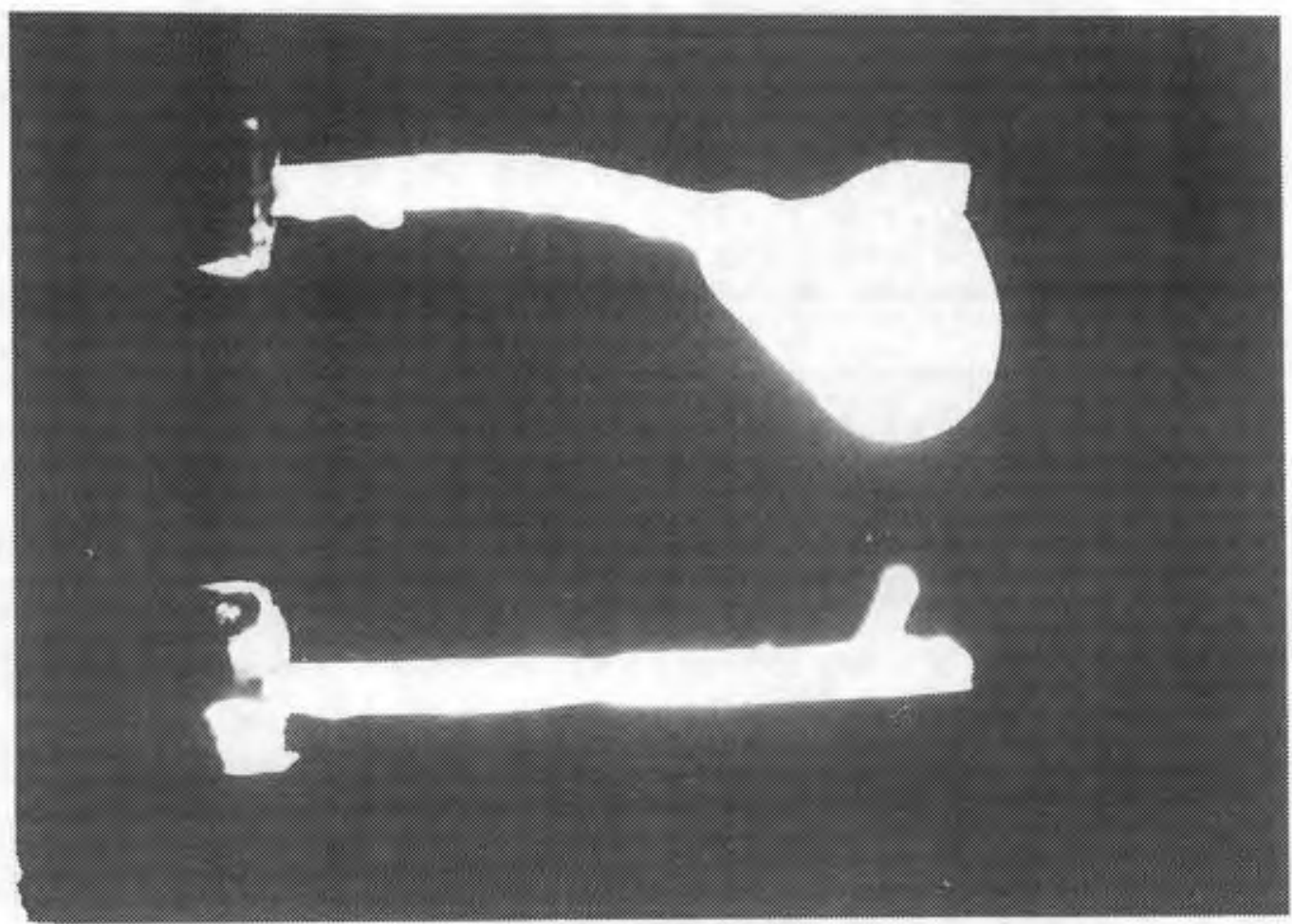

FIGURE 6.11

A pair of vessels in which the typical mild fusiform stenosis of the sleeve anastomosis and a large aneurysm of the ETEA vessel can be seen.

The measurements and calculated values for each of the 84 individual vessels (42 pairs) are tabulated in Appendix C. The analysis of the results is tabulated in Fig. 6.12.

The results show that the average cross sectional area (CSA) of the sleeve anastomosis is reduced by $1.61 \%$, while that of the ETEA is increased by $1.86 \%$. The difference between the diameters at the anastomotic site was statistically significant at the $p=0.05$ level. 


\begin{tabular}{|c|c|c|c|c|c|c|}
\hline $\mathrm{n}=42$ & \multicolumn{3}{|c|}{ ETEA } & \multicolumn{3}{c|}{ SIEEVE } \\
\hline \hline DIAM. (mm) & PROX & ANAS & DIST & PROX & ANAS & DIST \\
\hline \hline MAXIMUM & 1.237 & 1.257 & 1.242 & 1.249 & 1.251 & 1.256 \\
MINIMUM & 0.715 & 0.698 & 0.718 & 0.716 & 0.694 & 0.719 \\
AVERAGE & 0.999 & 1.008 & 1.004 & 1.008 & 0.999 & 1.013 \\
STD.DEV & 0.168 & 0.172 & 0.169 & 0.166 & 0.168 & 0.166 \\
\hline \hline CSA (mm $\left.{ }^{2}\right)$ & & & & & & \\
\hline \hline MAXIMUM & 1.181 & 1.223 & 1.190 & 1.219 & 1.206 & 1.231 \\
MINIMUM & 0.409 & 0.390 & 0.413 & 0.407 & 0.383 & 0.411 \\
AVERAGE & 0.806 & 0.821 & 0.814 & 0.819 & 0.805 & 0.827 \\
STD.DEV & 0.258 & 0.267 & 0.261 & 0.258 & 0.259 & 0.260 \\
\hline
\end{tabular}

FIGURE 6.12

Analysis of results obtained from measurements of corrosion casts.

The relative increase in diameter in the distal part of the vessels increased the CSA by $0.99 \%$ and $0.97 \%$ for the ETEA and the EIEA respectively. There was no statistical difference between these results.

\section{FLOW}

Flow was measured through 12 pairs of vessels with postoperative periods ranging from 1 to 32 days. The flow rates are shown in Fig. 6.13.

The flow rates in the early post-operative period were not significantly different. Both vessels permitted less flow than normal controls in rats of the same 


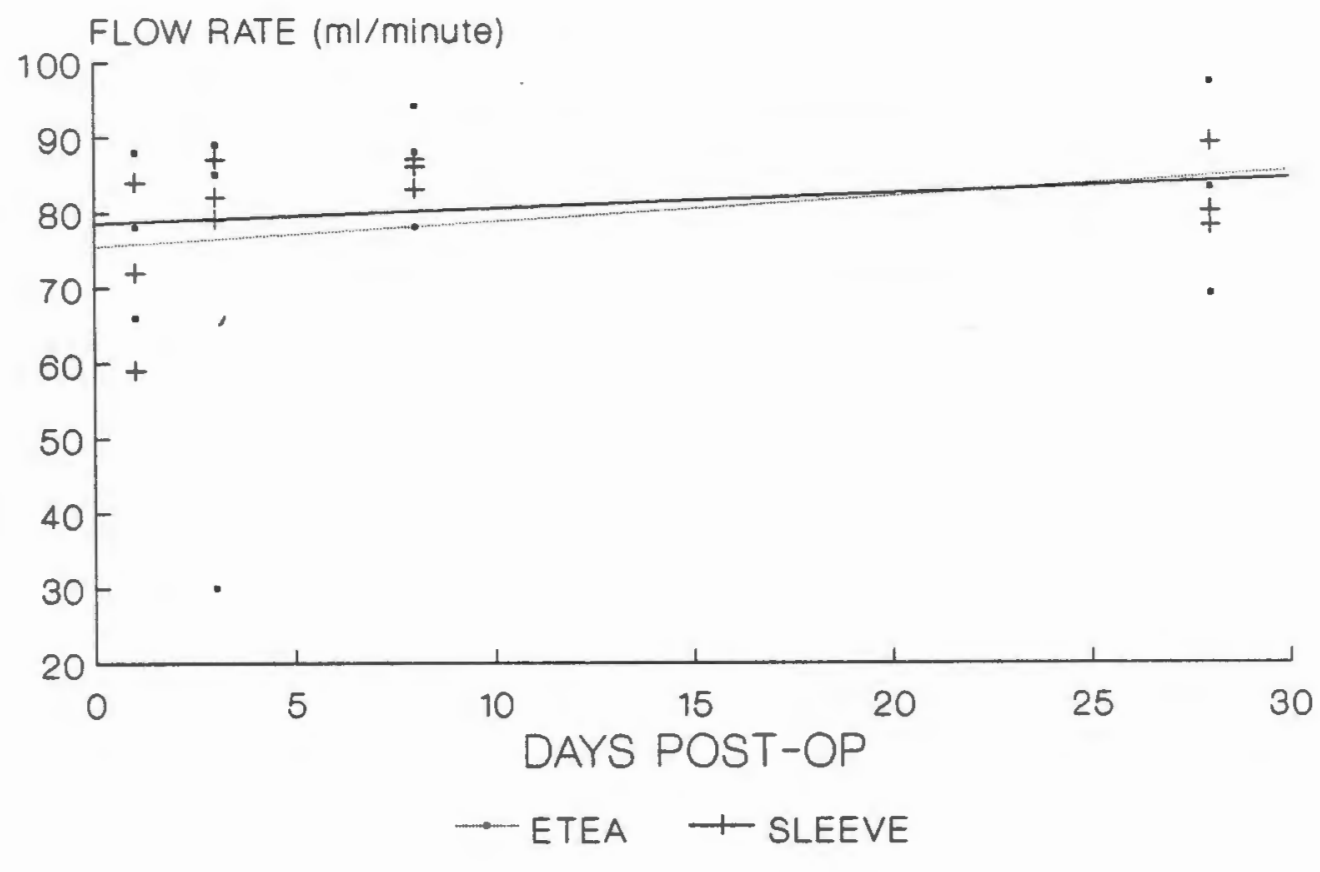

FIGURE 6.13

The flow rates in 12 pairs of vessels with postoperative periods ranging from 1 to 32 days.

mass. Flow through both vessels improved with time and after 7 days there were no significant differences between either of the anastomosed vessels and the controls.

\section{TENSILE STRENGTH}

Twelve pairs of anastomosed vessels and 4 controls matched for average body mass of the test animals were tested. The results are shown in Fig. 6.14. 


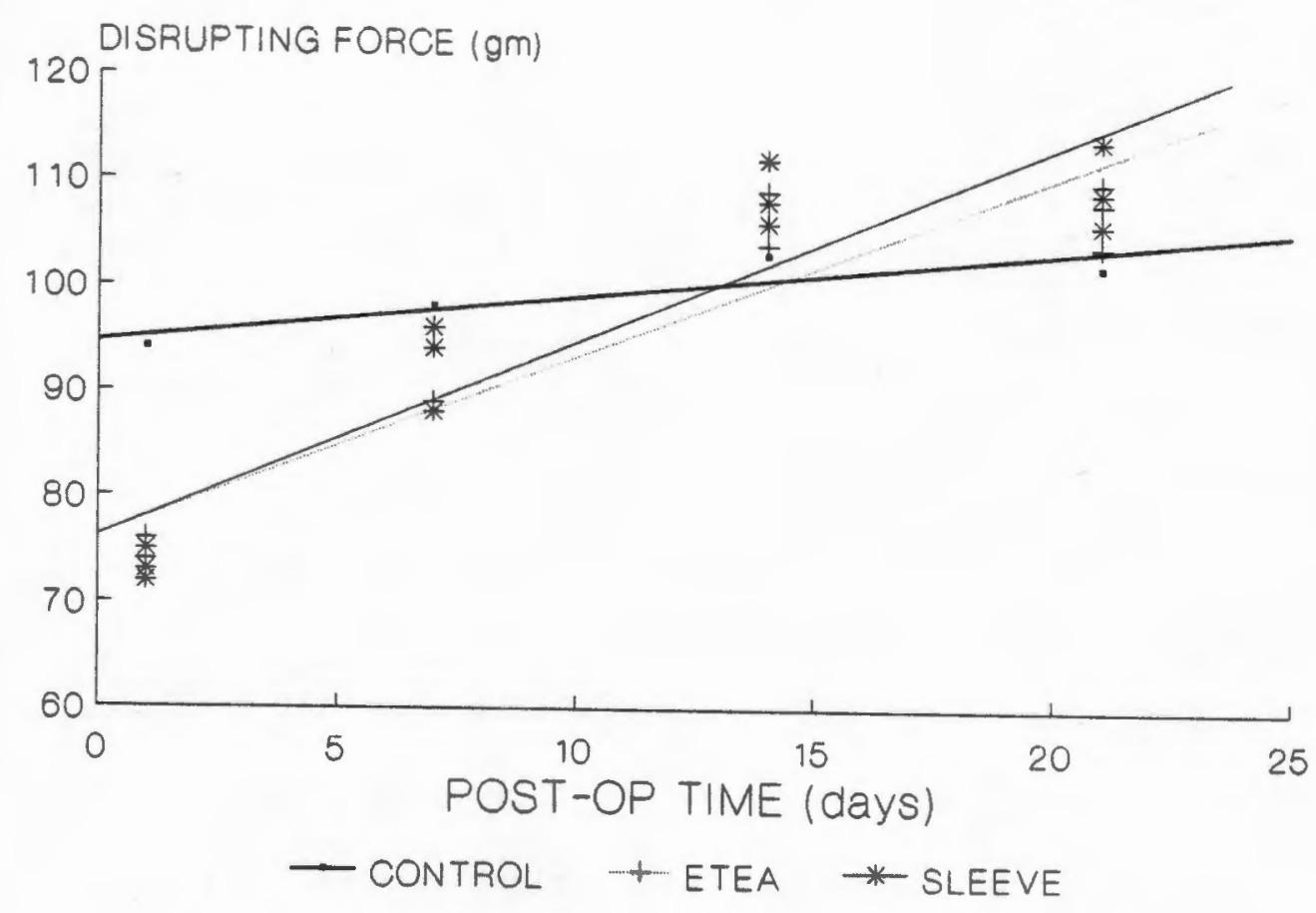

FIGURE 6.14

Tensile strength in rat femoral arteries

When tested by this method there was no significant difference between the tensile strengths of the anastomosed vessels at any stage in the healing period. Initially they were both weaker than the control vessels, but in the delayed group there were no significant differences between tests and controls.

\section{DISCUSSION}

I took much care in this comparative study to ensure that the vessels were subjected to identical conditions throughout the peri-operative, healing and testing periods. Many small and possibly insignificant factors were considered in the attempt to create equal conditions for the vessels, e.g. the ETEA which was used was the "posterior wall first" method of Harris, Finseth and Buncke. This method, like the sleeve anastomosis, does not require that the vessel be turned over during the anastomotic procedure. 
The finding that patency rates were not significantly different in these two anastomoses supports the findings of Lauritzen and Bagge (108) and Meyer et al (133) (see Fig. 5.6), who performed similar studies on their sleeve anastomoses.

The difference between the times taken for the two anastomoses was highly significant. I do not know of any other method by which the operating time has been as consistently and as markedly reduced.

The luminal topography of the sleeve anastomosis has been clearly demonstrated by the corrosion casting technique.

The flow study showed that the stenosis seen in the morphometric study made very little difference to the rheological function of the vessel. It is possible that the turbulence caused by the irregularities of the inner surface of the ETEA gives rise to a physiological stenosis, which is equal in rheological effect to the physical stenosis of the sleeve anastomosis.

The study of the tensile strength of the anastomosis, a factor which may be of considerable clinical importance to those surgeons who wish to encourage early post-operative movement in injured limbs or digits, has shown that the sleeve anastomosis is no weaker than the ETEA.

The findings of this series of comparative tests have therefore shown that, while the new sleeve anastomosis is no less safe or effective in any of the tested parameters, it is completed in a much shorter time.

Microsurgical operations notoriously take a long time to perform. A procedure which can safely save some time should therefore be welcomed. 


\section{NOVICE MICROSURGEONS: EIEA VS ETEA}

The EIEA, which has only two sutures, should theoretically present fewer opportunities for technical errors than an ETEA, in which 7 - 10 sutures are required. If the theory holds true, novice microsurgeons, who have not yet developed the dexterity required for microsurgical excellence, should have a higher success rate in performing EIEAs than with ETEAs.

\section{AIMS}

I aimed to establish the following facts concerning novice microsurgeons and the new EIEA:

1. Was their success rate significantly different with the EIEA, when compared with the ETEA ?

2. Was their operating time significantly reduced by the EIEA?

\section{METHODS AND MATERIALS}

Students who attended the courses in Basic Microsurgical Techniques (offered by the Microsurgery Laboratory of the Department of Surgery of the University of Cape Town Medical School) volunteered to participate.

The surgeons were from a variety of surgical disciplines and only one of them (BS, a foreign 5 thyear medical student) had had any previous microsurgical experience; none had had any previous formal training in microsurgical techniques.

The courses had a "hands-on" format. No more than 4 students were accepted in any particular course and there were always 2 instructors and 2 trained laboratory assistants available. This ensured a high level of individual attention to each student. Training was tailored to the needs and capabilities of each individual student around the following basic structure: 
DAY 1: Adjustment of the microscope, work surface and seating arrangement. Instrument handling, orientation in the operating field and instrument maintenance techniques. Basic suture handling, suture placement and knotting techniques, practised first on incisions in flat latex material and later on siliconerubber tubing.

DAY 2: Numerous anastomoses of a fresh rat aorta, which had been removed from a sacrificed animal by the laboratory staff and placed on a corkboard where it was held by bridging staples. Each anastomosis was critically evaluated by an instructor so that mistakes and incorrect methods were remedied at an early stage. Both the "stay-suture" (34) and "posterior wall first" (76) techniques were taught.

DAY 3: Students were provided with anaesthetised adult laboratory rats in which the abdominal aortae were to be dissected and anastomosed. Emphasis was placed on the tissue handling and dissection techniques. When this had been completed, the operative approach, dissection and ETEA of the rat femoral artery was demonstrated by the instructor and then followed on the contralateral side by the student.

DAY 4: Each student performed 2-3 ETEAs in a rat femoral artery. End-to-side anastomosis of femoral artery to femoral vein was demonstrated by the instructor and followed on the contralateral side by the student. EIEA was then demonstrated by the instructor and again followed on the contralateral side by the student. 
DAY 5: Students were free during morning to choose any type and to perform any number of anastomoses. All procedures were timed by laboratory staff, from vessel division to final clamp release.

Students who had volunteered to participate in the study were then asked to perform a new EIEA in one femoral artery and an ETEA in the contralateral vessel. Both of these anastomoses were carefully timed by observers. The wounds were closed and the animals given a $3 \mathrm{ml}$ subcutaneous fluid replacement injection and allowed to recover from the anaesthetic.

The animals were kept under normal conditions for anything up to 20 days and were then evaluated under anaesthetic by direct examination of the anastomoses. Thereafter they were killed and the vessel segments were removed for further study.

\section{RESULTS}

The results are shown in Fig. 6.15 to Fig. 6.18 .

\begin{tabular}{|c|c|c|c|c|}
\hline ANASTOMOSIS & $(\mathrm{n})$ & PATENT & FAILED & $\%$ FAIL \\
\hline \hline SLEEVE & 37 & 35 & 2 & $5.41 \%$ \\
ETEA & 41 & 29 & 12 & $29.26 \%$ \\
\hline TOTAL & 78 & 64 & 14 & $17.95 \%$ \\
\hline
\end{tabular}

FIGURE 6.15

Patency rates compared in vessels anastomosed by novice microsurgeons.

There was a significant difference between the patency rates of the two methods $\left(X^{2}=6.19\right.$; $\mathrm{df}=1$, thus $P<0.05$ ). 
Twelve pairs of anastomoses performed by 10 different surgeons were timed. The results are shown in Fig. 6.16.

\begin{tabular}{||c|c|c|c|c|c|c||}
\hline SURGn & SLEEVE & TIME & ETEA & TIME & EVAL & PAT \\
\hline \hline (init) & $Y /-$ & (min) & $Y /-$ & (min) & days & $Y / N$ \\
\hline \hline SS & - & - & $Y$ & 70 & 0 & $\mathrm{~N}$ \\
SS & $Y$ & 46 & - & - & 0 & $Y$ \\
PN & $Y$ & 22 & - & - & 13 & $Y$ \\
PN & - & - & $Y$ & 36 & 13 & $Y$ \\
BS & $Y$ & 11 & - & - & 13 & $Y$ \\
BS & - & - & $Y$ & 27 & 13 & $Y$ \\
JT & $Y$ & 26 & - & - & 13 & $Y$ \\
JT & - & - & $Y$ & 29 & 13 & $Y$ \\
TDC & $Y$ & 27 & - & - & 13 & $Y$ \\
TDC & - & - & $Y$ & 55 & 13 & $Y$ \\
TDC & $Y$ & 20 & - & - & 14 & $Y$ \\
TDC & - & - & $Y$ & 53 & 14 & $Y$ \\
MW & $Y$ & 31 & - & - & 14 & $Y$ \\
MW & - & - & $Y$ & 57 & 14 & $Y$ \\
DB & $Y$ & 35 & - & - & 6 & $Y$ \\
DB & - & - & $Y$ & 90 & 6 & $Y$ \\
DB & - & - & $Y$ & 35 & 0 & $\mathrm{~N}$ \\
DB & $Y$ & 26 & - & - & 22 & $Y$ \\
KC & - & - & $Y$ & 86 & 2 & $\mathrm{~N}$ \\
KC & $Y$ & 25 & - & - & 0 & $Y$ \\
PC & $Y$ & 16 & - & - & 19 & $Y$ \\
PC & - & - & $Y$ & 25 & 19 & $Y$ \\
PP & $Y$ & 15 & - & - & 19 & $Y$ \\
PP & - & - & $Y$ & 25 & 19 & $Y$ \\
\hline \hline
\end{tabular}

FIGURE 6.16

Novice microsurgeons' timed pairs of anastomoses.

The analysis of these times is tabulated in Fig. 6.17. 


\begin{tabular}{|c|l|c|c|c|c|}
\hline \hline ANASTOMOSIS & $(\mathrm{n})$ & MAX. & MIN. & MEAN & S.DEV. \\
\hline \hline SLEEVE & $(12)$ & 46 & 11 & 23.0 & 10.99 \\
ETEA & $(12)$ & 90 & 25 & 45.2 & 25.15 \\
\hline
\end{tabular}

FIGURE 6.17

Analysis of Novice operating times.

Applying the $t$-test for small correlated samples to these data yielded the following results:

sd diff $=5.28 S E_{\text {diff }}=1.59 \quad$ Mean diff $=24$ therefore $t=15.07 \quad(d f=11) \quad P<0.005$

The difference in times taken for the two anastomoses is therefore significant at the $P<0.005$ level.

The times taken by individual surgeons for the different types of anastomoses are plotted against each other in Fig. 6.18 and they are fairly well correlated $\left(r=0.68 ; n_{p}=12\right.$; hence $P<$ $0.05)$.

Vessels which were occluded at follow-up examination were all found to contain intraluminal thrombi. In 6 of the ETEAs there were occlusive sutures which caught the opposite wall. In 4 cases there were definite signs of intimal separation and primary occlusion of the lumen by an intramural haematoma. Sutures which had not traversed all the layers of the vessel wall could be clearly seen in these specimens. In the remaining two ETEA failures there was no obvious cause for the thrombosis, but adventitial intrusion was suspected, as the sutures were irregularly spaced. 


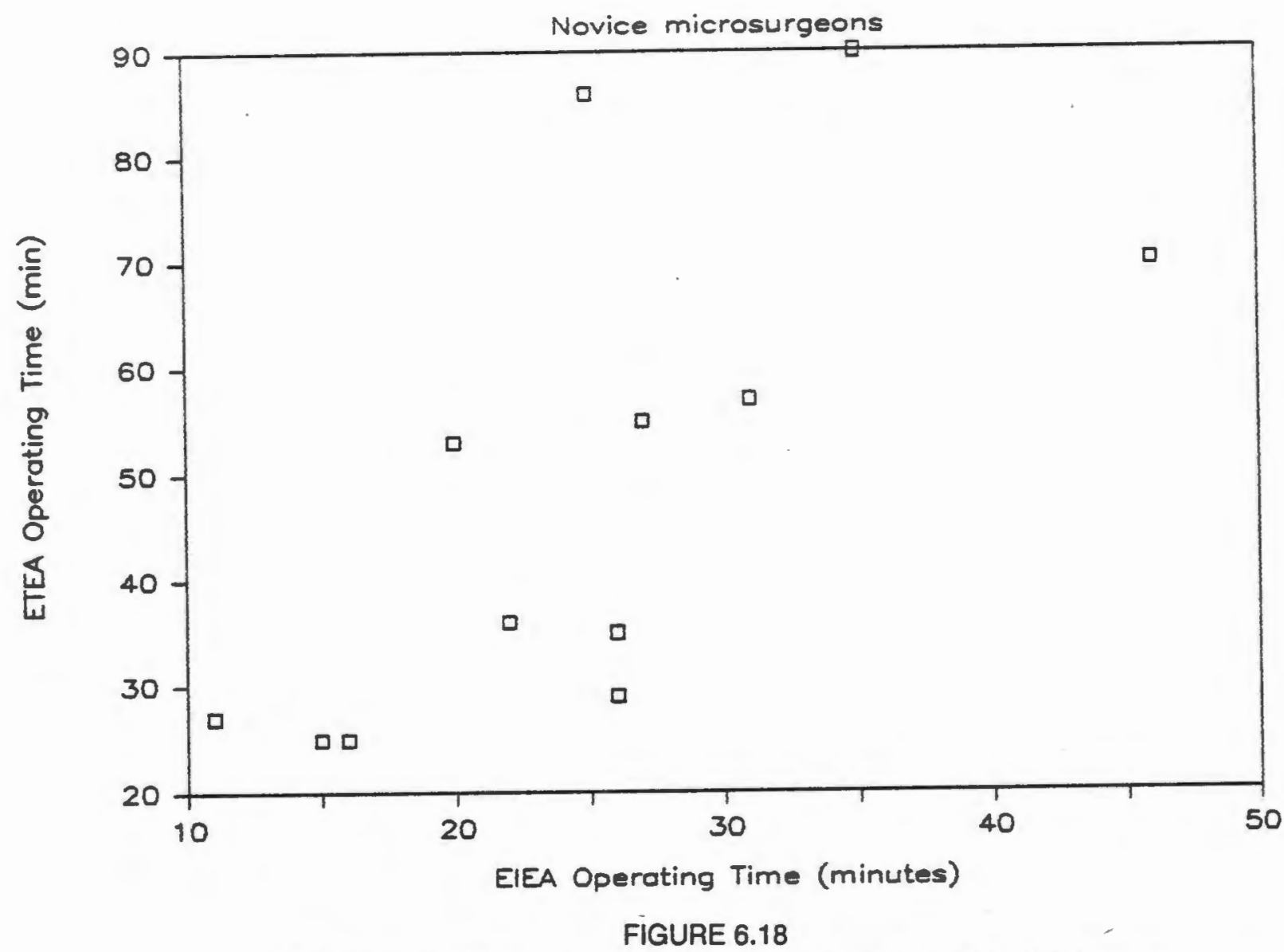

EIEA time vs. ETEA time showing correlation due to variable skill factors.

The failed sleeve anastomoses both contained thrombus. In one case the space between the entry and the exit of the first deep loop through the wall of the RV had been very wide. This caused puckering under the tied suture. In the second case the FV's adventitia was clearly excessive and it had probably initiated thrombosis by intruding into the blood stream.

\section{DISCUSSION}

The results of this limited study in the employment of two different types of anastomoses by novice microsurgeons indicated that surgeons, at this level of experience, performed the sleeve anastomosis more effectively than the ETEA, when evaluated by the parameters of operating time and patency. 
It was necessary for students to acquire their basic skills by first performing a number of standard ETEAs. In doing so they would obviously have had more opportunities of performing the basic movements of needle control, suture placement and knot tying. Having therefore practised at many more ETEAs than at sleeve anastomoses, they might have been expected to perform better at the ETEA.

There was a high positive correlation between the times taken by individual surgeons for the two different anastomoses. This is probably due to the fact that some individuals are more suited to microsurgery than others and that individuals themselves have good and bad days for performing microsurgical operations. When a surgeon is correctly attuned and in possession of the necessary basic abilities, his performance will be more efficient than that of the surgeon who lacks one or more of the required attributes, regardless of the type of operation.

The significance of the differences in the results of the different types of anastomoses, performed by surgeons who had not yet acquired polished microsurgical skills, raises the question as to whether similar differences would not apply in the case of microsurgeons who had lost the "polished surface" of their skills by infrequent use. If this is the case, the sleeve anastomosis may be a most useful tool for the surgeon who has to manage a diversity of interventive skills in his practice in outlying areas. 


\section{SUMMARY}

Novice microsurgeons undergoing their first week of microsurgical training were taught first the standard ETEA and then the new sleeve anastomosis. Patency rates in the sleeve anastomoses (35/37 ie 94.5\%) were significantly higher $(P<0.05)$ than in the ETEAs $(29 / 41$ ie $70.7 \%)$. The time taken for the sleeve anastomosis was significantly less $(P<0.005)$ than that taken for the ETEA. It may be concluded that, judging by these parameters, novice microsurgeons perform the sleeve anastomosis more effectively than the ETEA. 
Chapter 6- Comparative Studies 


\title{
CHAPTER VII. \\ ULTRASTRUCTURE
}

\author{
To see a world in a grain of sand \\ And heaven in a wild flower, \\ Hold Infinity in the palm of your hand \\ And Eternity in an hour.
}

William Blake (c. 1800)

\section{SEM STUDY OF THE HEALING EIEA}

\section{AlMS}

Other workers have previously studied the healing of standard microvascular anastomoses and sleeve anastomoses by means of standard histological methods and by SEM. The findings of those who studied the sleeve anastomoses were very similar (see description below) and there was no reason to believe that there would be any significant difference in the healing of the new sleeve anastomosis if it was examined by the same methods. However, the preparation of small vascular specimens for SEM examination is not standardised and little is known about the artifactual distortions which might have been caused by any of the methods which have been used.

In this study I observed the healing process in the sleeve anastomosis by means of SEM while using a very simple method of preparation. 


\section{METHODS AND MATERIALS}

Adult male Long-Evans rats $(300 \mathrm{~g}-400 \mathrm{~g})$ were prepared in the same way as those in the comparative study (see Chapter 6) except that sleeve anastomoses were performed in both femoral arteries.

Based on the times at which the most significant changes in the healing process had been observed in other studies $(100,120,196), 1$ elected to examine the anastomoses at 1 hour, 3 hours and at $1,3,8$ and 28 days.

At the appropriate evaluation times the animals were prepared as for corrosion-casting, according to the instructions set out in Appendix B, up to the point where they were completely exsanguinated and ready for injection of the resin. The flushing solution was Ringer's lactate in all cases.

The aorta was ligated distal to the renal arteries. A cannula was placed distal to the ligature and $20 \mathrm{ml}$ of a $3 \%$ buffered glutaraldehyde solution was injected through this cannula into the aorta to flush and fix the femoral arteries. Vascular clamps were applied to the femoral artery at distances of $1.5 \mathrm{~mm}$ on either side of the anastomosis during the last part of the glutaraldehyde injection. The distal clamp was applied first and the proximal clamp followed while maintaining the injection pressure of $100 \mathrm{~mm} \mathrm{Hg}$. The fixative solution was allowed to remain in the vessels for 1 hour. 


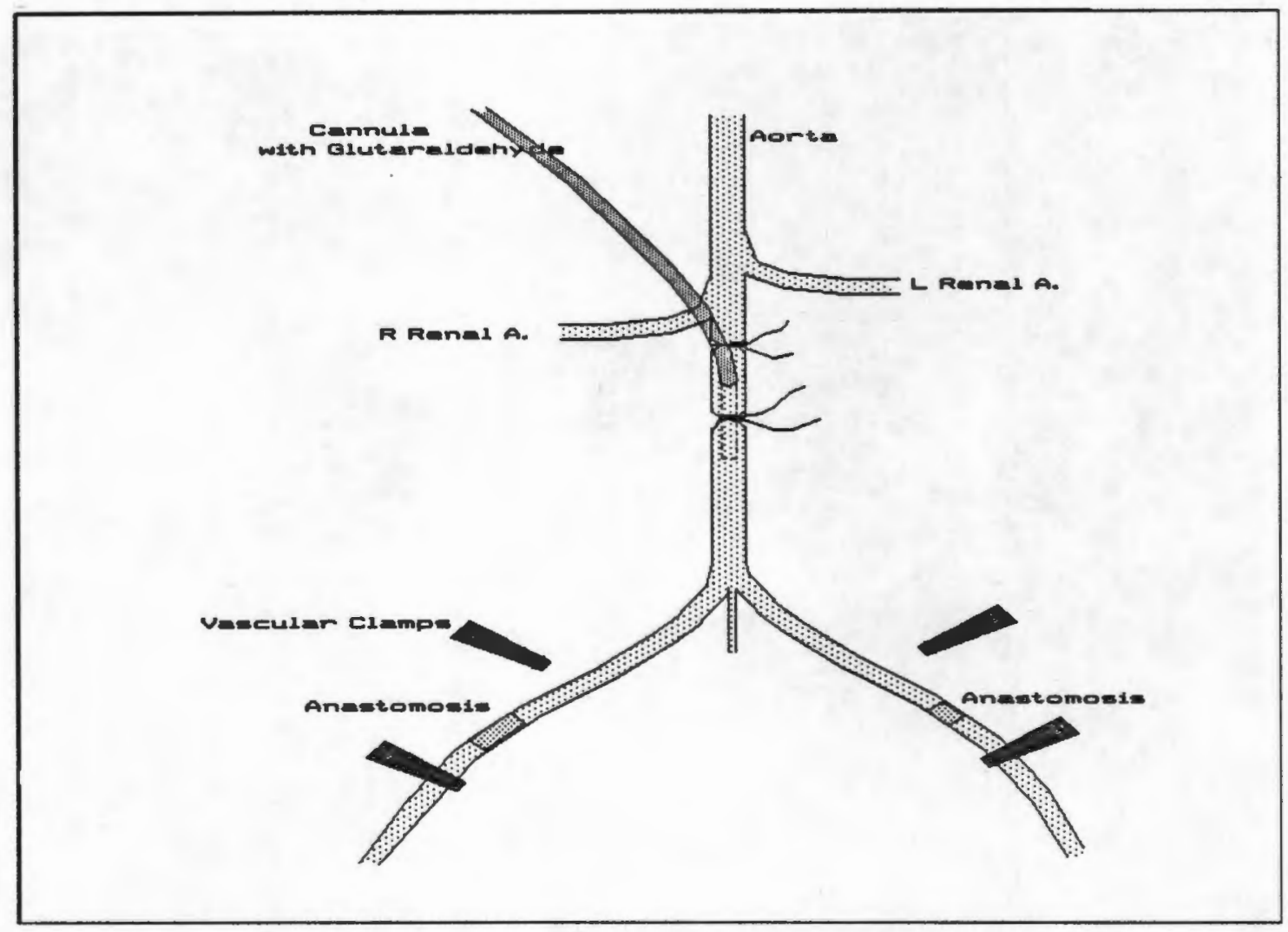

FIGURE 7.1

Anatomical preparation for obtaining vascular specimens for SEM.

After the glutaraldehyde flush and the 60-minute fixation period the femoral arteries were extirpated. Under $40 \mathrm{X}$ magnification of the operating microscope one vessel was cut transversely near the anastomotic site and the contralateral vessel was cut longitudinally through the overlap area. The sections of the vessels were dropped into small containers of liquid nitrogen which were placed in a bath of the same cooling substance. The small specimens were thus instantaneously frozen. The specially designed small containers (Fig. 7.2) were taken in their liquid nitrogen bath to the freeze-drying apparatus, where the nitrogen was removed and the containers vacuum-sealed. 


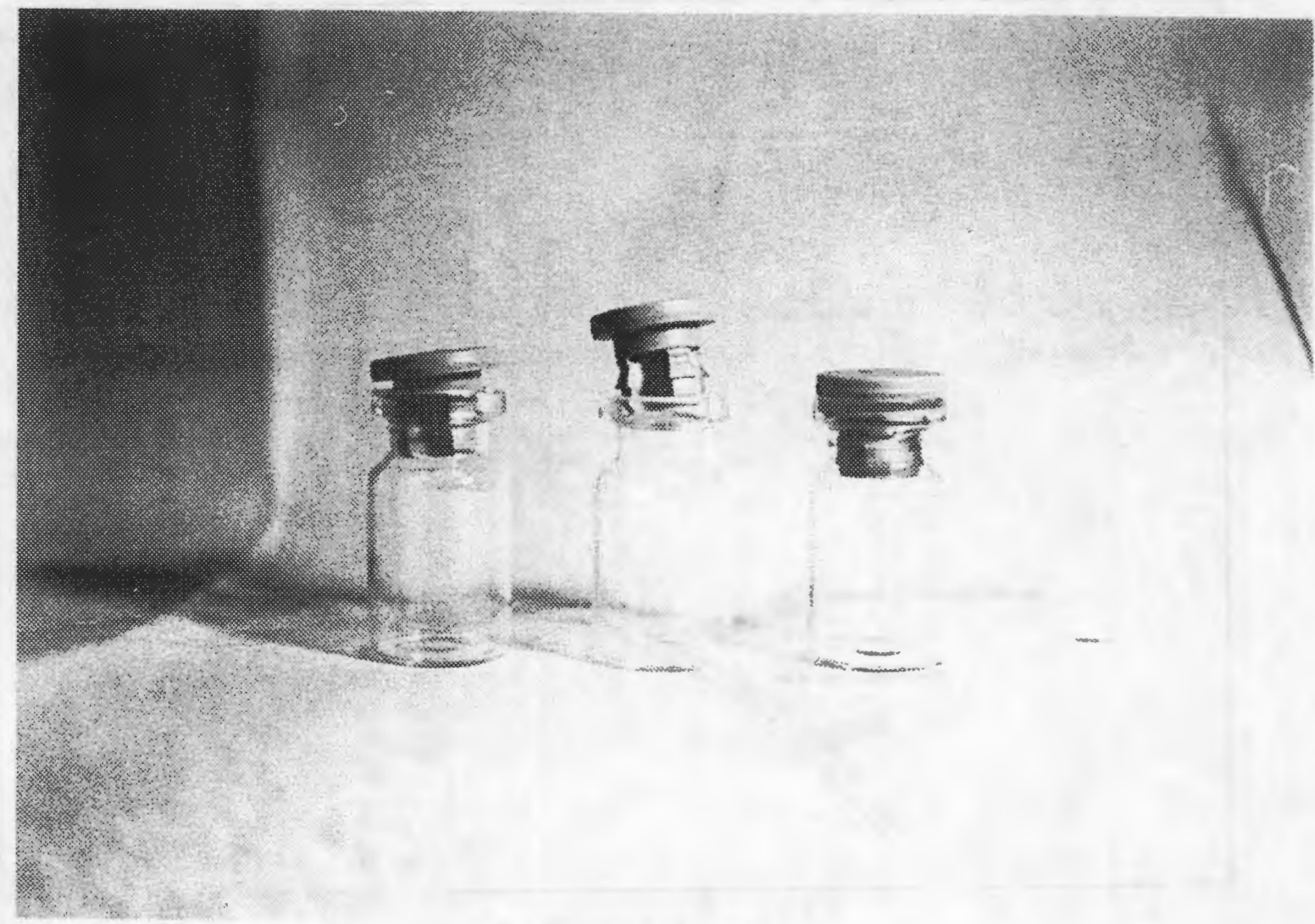

FIGUAE 7.2

The special container for freeze-drying specimens.

The freeze-dried sections of both specimens were mounted on a single aluminium SEM stub (using a graphite/glue mixture), placed in a dessication chamber for 24 hours and then goldpalladium coated in a sputter-coater for 4 minutes. They were then examined by means of a Cambridge $\$ 200$ Scanning Electron Microscope. Photomicrographs were obtained on llford FP4 roll film.

\section{RESULTS}

The first few hours following anastomosis were characterised by deposition of platelets, red cells and leucocytes over the uncovered area of the FV's end. The cellular and particulate elements appeared to pave the uncovered area in a thin layer. 
After a day the adherent cells appeared well fixed to the substrate by fibrin deposits creating the appearance of a well organized cobblestone pavement. The step made by the end of the FV within the RV had become filled in with a sloping deposit of cells and fibrin (see Fig. 7.4). Endothelial cells were noted to be growing over this pavement at the edges.

At three days the endothelium had bridged parts of the pavement and at 8 days there was complete cover in all but one of the six vessels I examined.

By 28 days the endothelial surface had largely regained its normal appearance and it was difficult in some cases to find the edge of the FV by SEM of the luminal surface.

Some of the SEM photomicrographs obtained are shown below. 


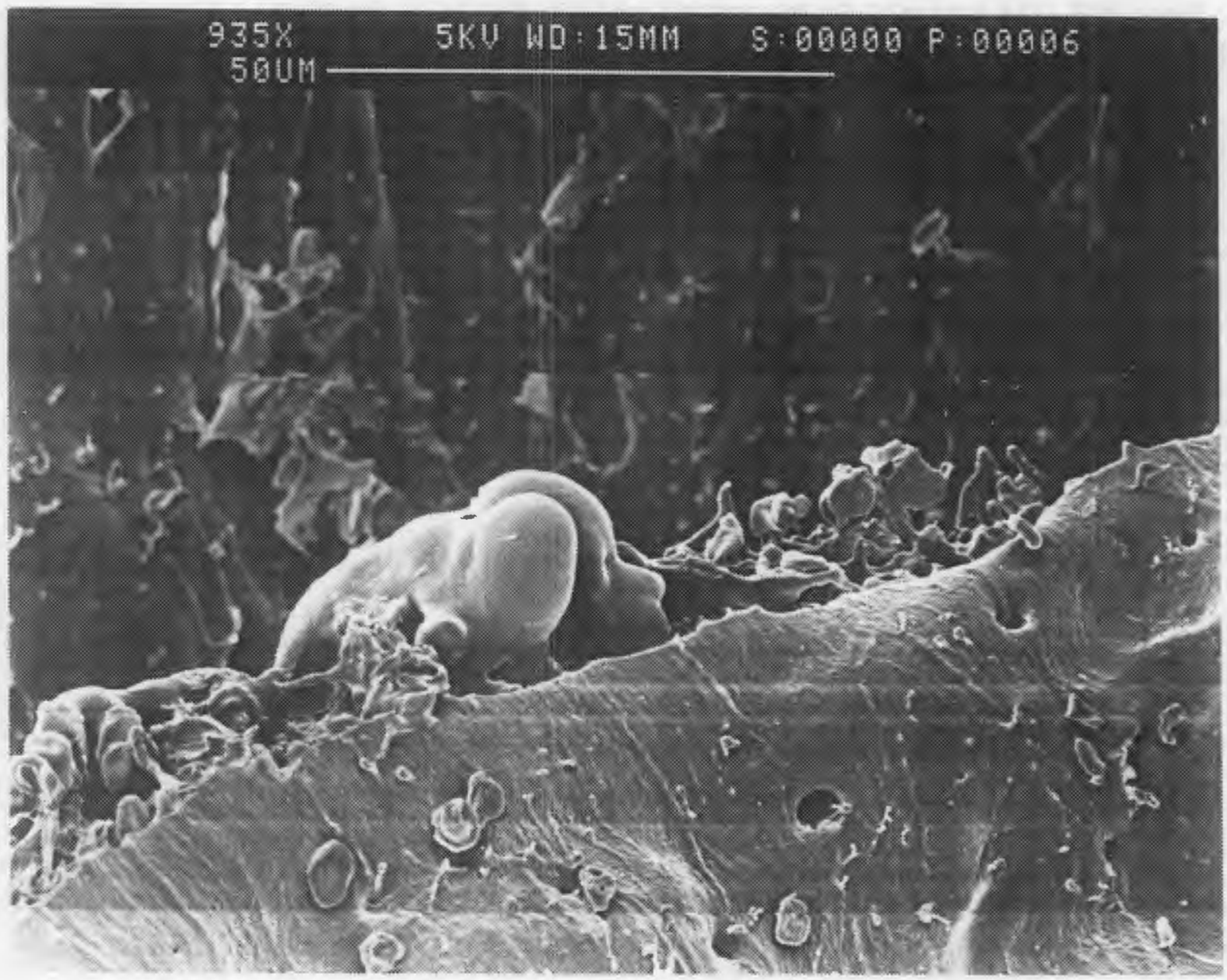

FIGURE 7.3

The sleeve anastomosis at 3 hours, looking from within the distal end of the FV with the endothelial surface of the FV lying in the foreground. The the inner edge of the "step area", to which a number of red cells, platelets and fibrin strands and a large leucocyte have become adherent, can be clearly seen. In the background lies the endothelial surface of the RV. Note that the endothelialised surfaces are relatively free of adherent cells and debris. 
Chapter 7 - Ultrastructure

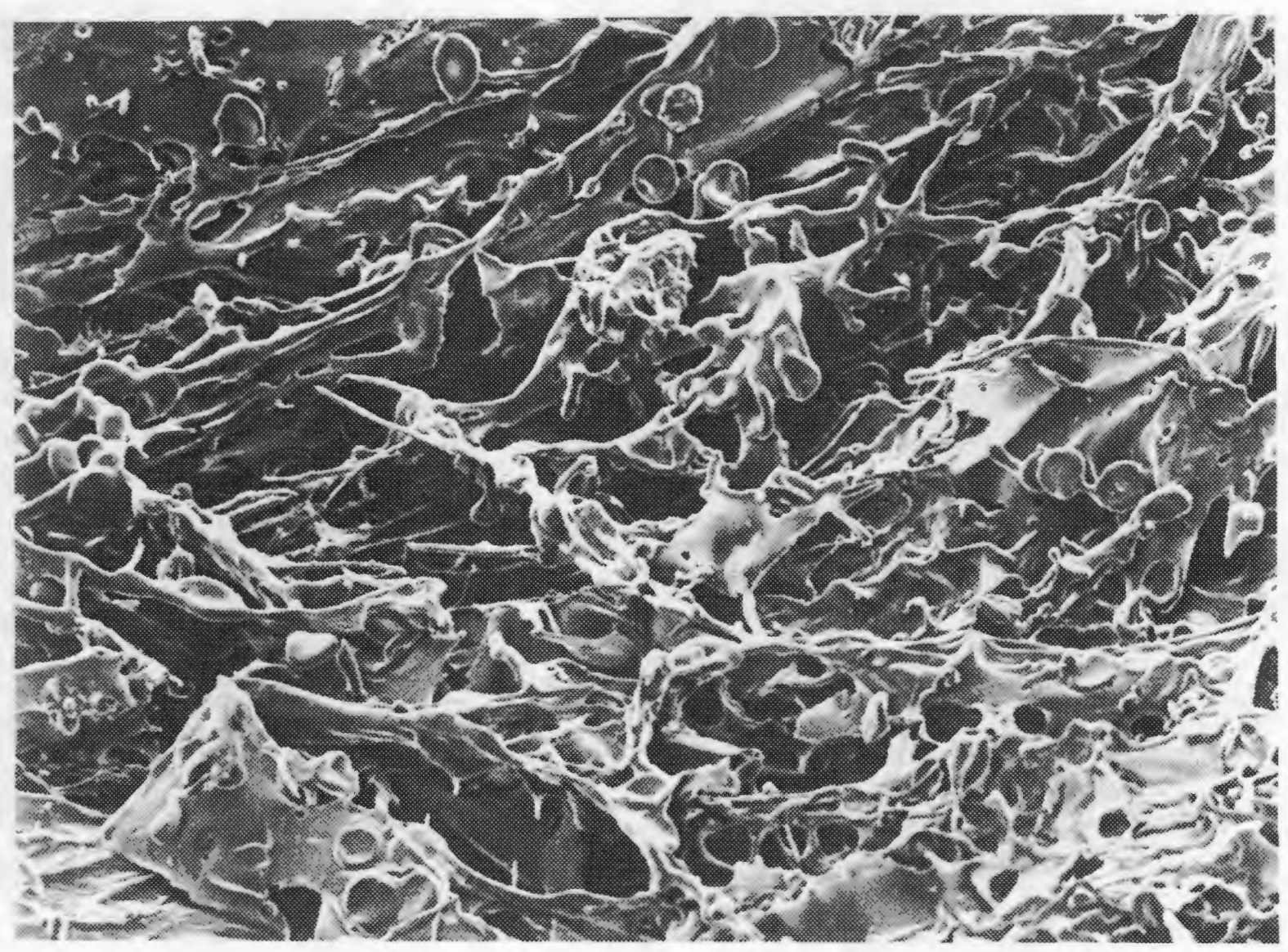

FIGURE 7.4

The step between the endothelial surfaces of the FV and the RV is filled with cellular and particulate deposit. 


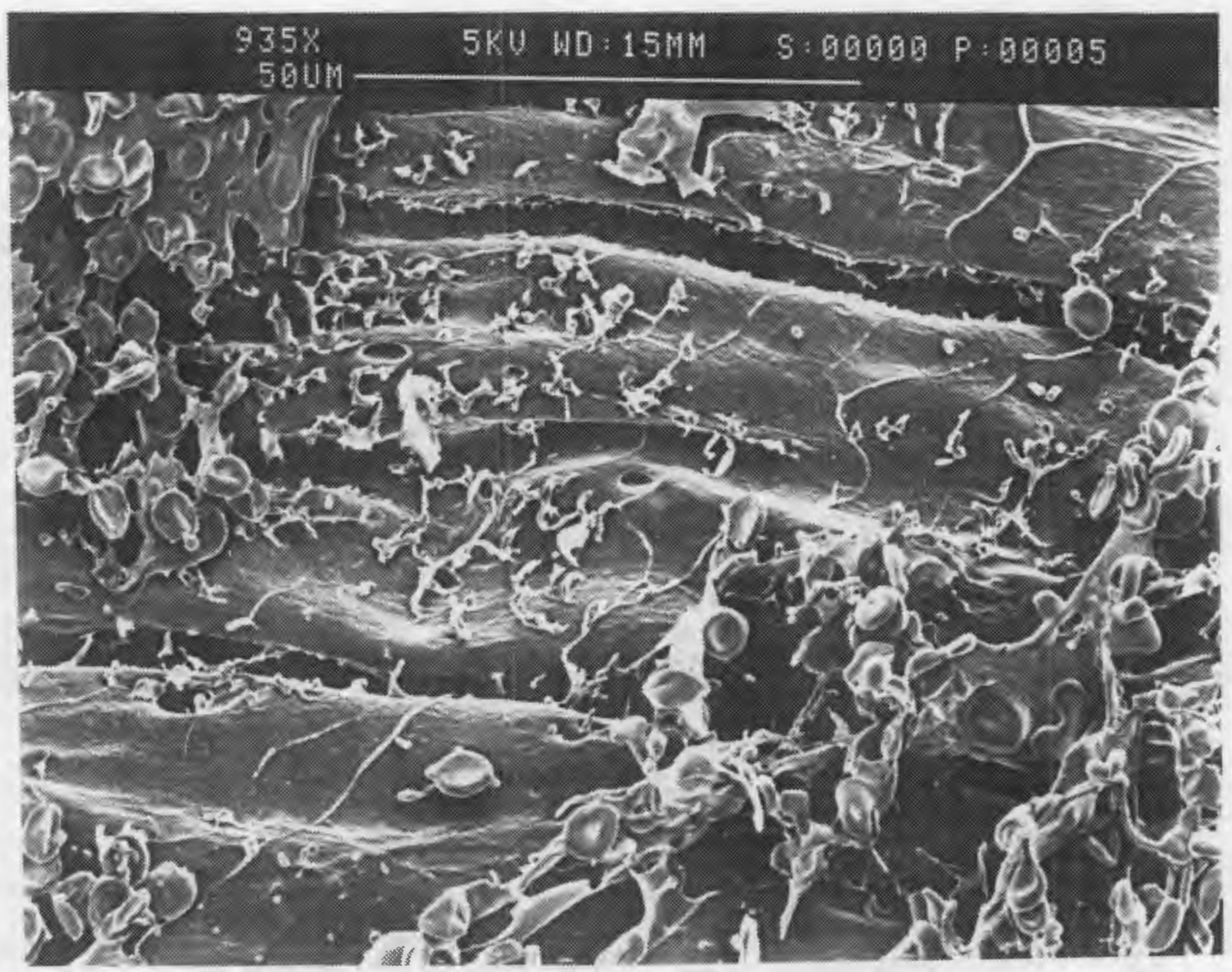

FIGURE 7.5

After 8 days endothelial cells have grown over clot in the step area. The endothelial lining is almost complete in this area. 


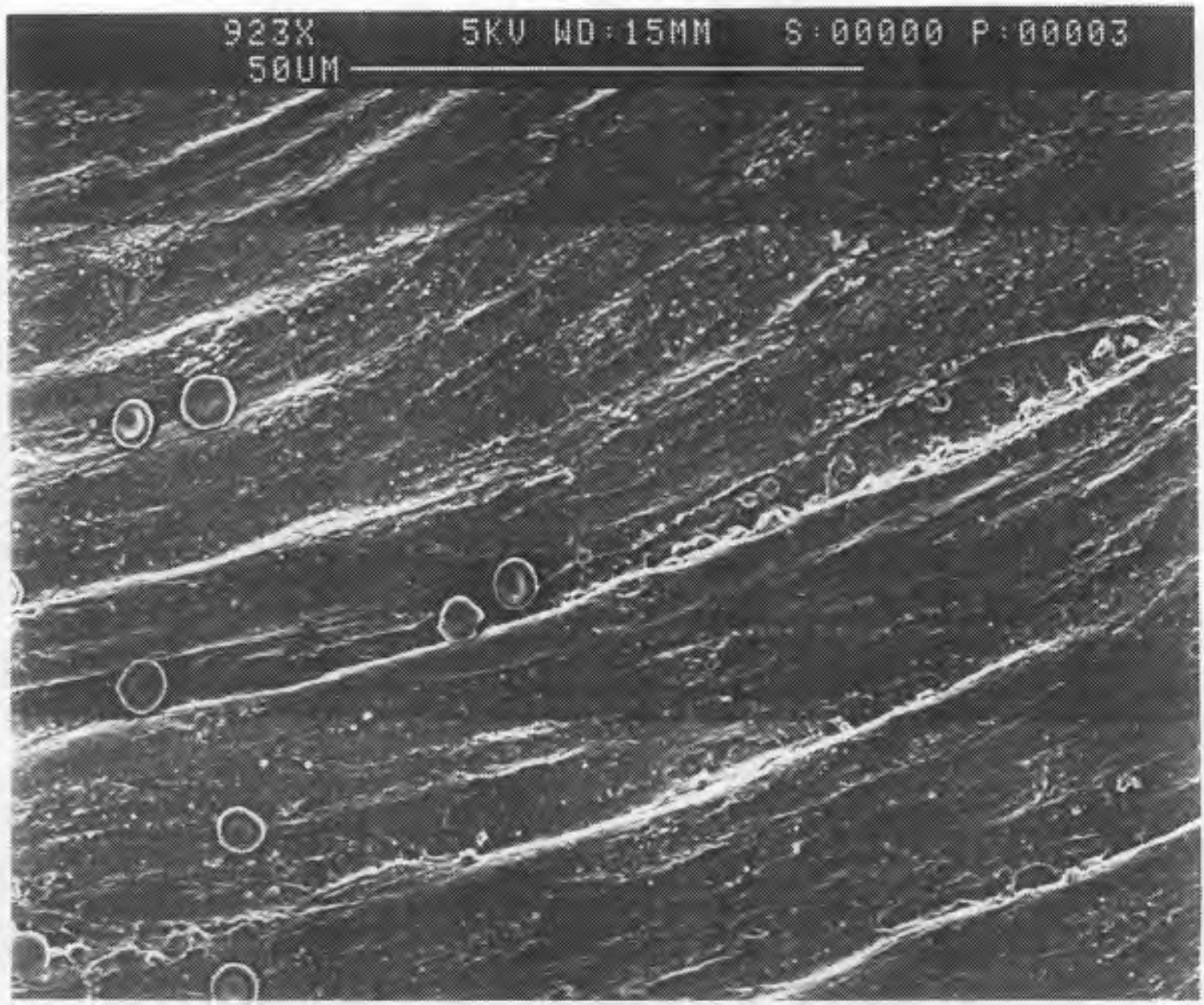

FIGURE 7.6

The endothelial surface overlying the step area at 28 days. The endothelial surface is flatter and smoother than normal, possibly as a result of the fact that it is overlying a substrate of cellular debris rather than a normal media. 


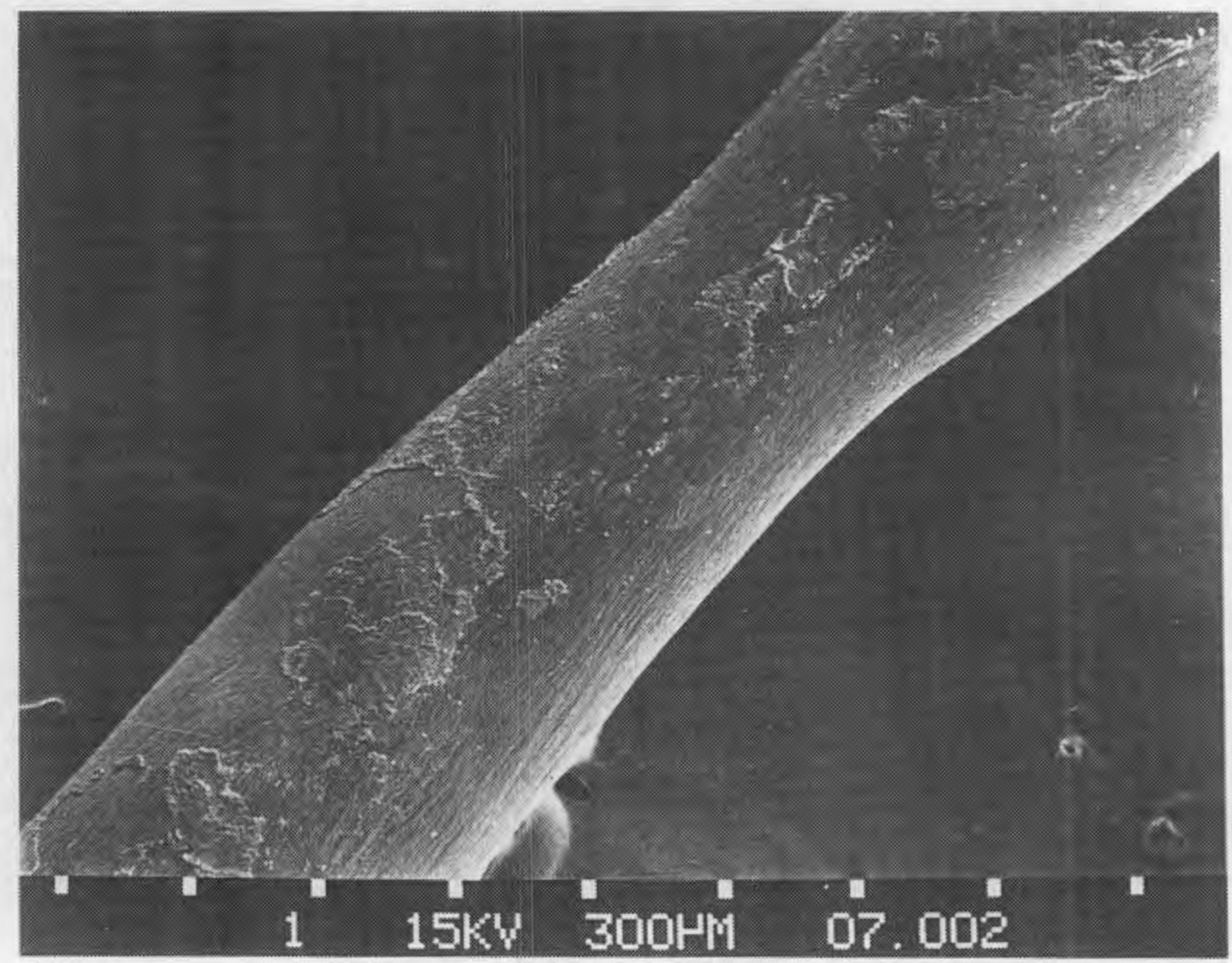

FIGURE 7.7

A SEM photomicrograph of a corrosion cast of a sleeve anastomosis in the rat femoral artery at 68 days post operation. The surface of the cast is smooth and the impressions made by the rugae of the endothelial surface are demonstrated as is the mild fusiform stenosis characteristic of the sleeve anastomosis. The adherent debris on the surface of the cast is due to incomplete corrosion of the vascular tissue. 


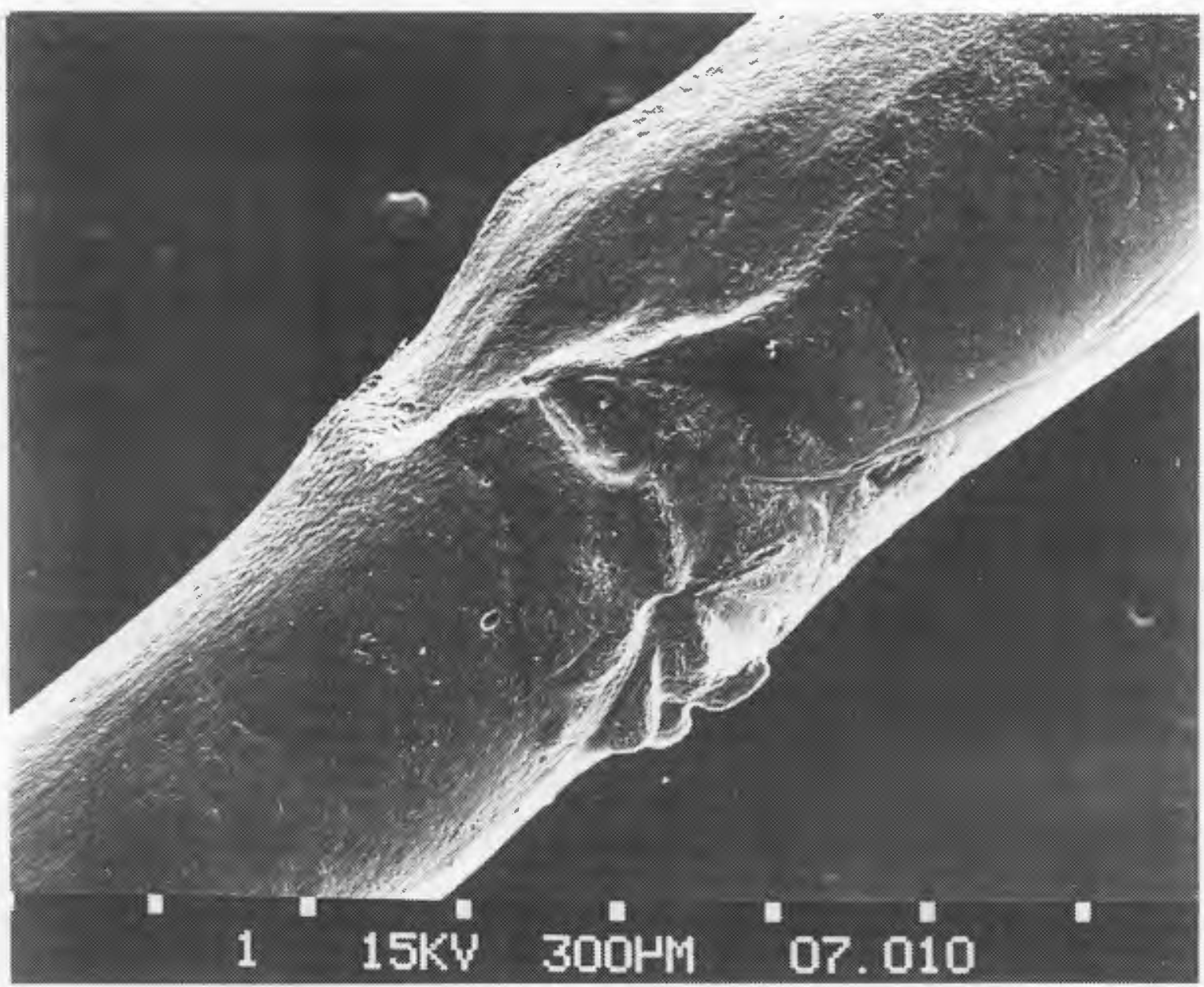

FIGURE 7.8

The contralateral vessel of the same animal (Fig 7.7) was anastomosed by means of an ETEA. The casts were made simultaneously. This cast demonstrates the typical surface irregularity seen within an ETEA despite meticulous execution of the anastomosis. 


\section{DISCUSSION}

Krag et al found that healing of the sleeve anastomosis proceeded in much the same way as that of an ETEA. During the early postoperative period there is an accumulation of cells and platelets in the vicinity of the internal overlap step. They adhere to the non-endothelialised areas and "pave" those exposed surfaces. The lumen is narrowed in the overlap area. The endothelium begins to grow and advances over the aggregate, covering it completely within less than 7 days. The media usually becomes necrotic in the ETEA and they mention the possibility that this necrosis may occur throughout that portion of the vessel which lies between the clamps during the anastomosis. In the EIEA they found necrosis limited to part of the media of the outer vessel. The area in which this necrosis occurs is subsequently replaced by fibrous scar tissue.

Lauritzen et al carried out a careful study of the healing EIEA (109). They injected $2 \%$ Evans Blue Solution intravenously before sacrificing the animal in order to demonstrate cell damage in the intima by dye uptake. They then examined the vessels by means of the operating microscope to observe the amount of blue staining and thereafter continued preparation for SEM examination. Their study showed exactly the same series of events which I have observed. They also noted that after three days there seemed to be an improved adaptation between the vessel diameters i.e. the stenosis seen in the first 2 days had decreased. I question the possibility that this may be linked to the necrosis of the outer vessel's media observed by Krag et al (100).

Is it perhaps important to the long-term function of the EIEA that the media of the RV should necrose and thus allow the luminal diameter of the FV to approach that of the RV?

The SEM study of the healing of the new sleeve anastomosis has shown that the healing process proceeded in much the same way as was previously described for other sleeve anastomoses by Lauritzen (109) and Krag (100). 


\section{THE EIEA IN THE GROWING VESSEL}

\section{AlMS}

In theory the sleeve anastomosis should be of special benefit in the growing vessel as there are only two points of scarring in the vessel wall.

I therefore wished to study the effect of the new sleeve anastomosis on the growth of a vessel.

\section{METHODS AND MATERIALS}

The sleeve anastomosis was performed on the right femoral arteries of 10 young, male LongEvans rats, weighing approximately $150 \mathrm{~g}$. All preparations and methods were the same as those previously described for the comparative study in Chapter 6 . The vessels were approximately $0.5 \mathrm{~mm}$ in diameter (see Fig. 7.10 ).

The animals were allowed to treble their operative weight before evaluation, which was performed by means of the corrosion-casting system described in Appendix B. 


\section{RESULTS}

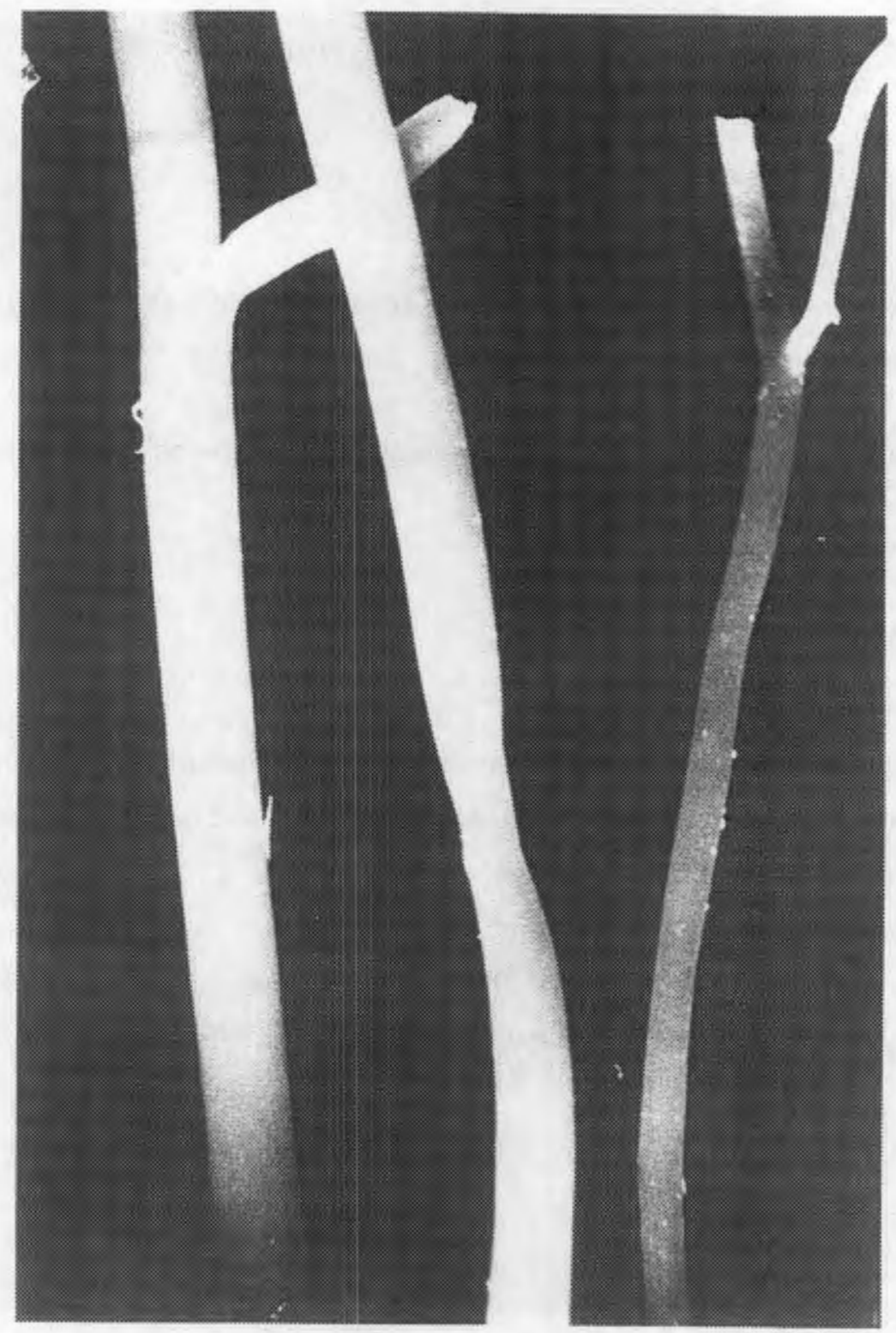

FIGURE 7.9

Corrosion-casts of vessels, viz.: $[A]$ which has grown after anastomosis, $[B]$ the normal control from the opposite limb of the same animal and [C] the femoral artery of a $150 \mathrm{~g}$ animal.

The results of the specimen measurements, which were performed in the same way as those in Appendix B, are tabulated in Fig. 7.10. 


\begin{tabular}{|c|c|c|c|c|c|c|}
\hline \multicolumn{4}{|c|}{ AT OPERATION } & \multicolumn{3}{|c|}{ AT EVALUATION } \\
\hline No. & $\begin{array}{r}\text { MASS } \\
(g \mathrm{~m})\end{array}$ & DIA & $\begin{array}{l}\text { IETER } \\
\text { Im) } \\
\text { L }\end{array}$ & $\begin{array}{c}\text { MASS } \\
(\mathrm{gm})\end{array}$ & $\underset{\mathbf{R}}{\text { DIAM }}$ & a) \\
\hline $\begin{array}{r}1 \\
2 \\
3 \\
4 \\
5 \\
6 \\
7 \\
8 \\
9 \\
10\end{array}$ & $\begin{array}{l}146 \\
153 \\
156 \\
152 \\
147 \\
149 \\
154 \\
158 \\
152 \\
148\end{array}$ & $\begin{array}{l}.50 \\
.50 \\
.55 \\
.50 \\
.45 \\
.50 \\
.50 \\
.55 \\
.50 \\
.50\end{array}$ & $\begin{array}{l}.50 \\
.50 \\
.55 \\
.50 \\
.45 \\
.50 \\
.50 \\
.55 \\
.50 \\
.50\end{array}$ & $\begin{array}{l}449 \\
452 \\
476 \\
456 \\
447 \\
459 \\
448 \\
479 \\
452 \\
450\end{array}$ & $\begin{array}{r}1.10 \\
1.00 \\
1.20 \\
1.00 \\
.90 \\
1.00 \\
.80 \\
1.20 \\
1.00 \\
.90\end{array}$ & $\begin{array}{r}1.10 \\
1.10 \\
1.10 \\
1.00 \\
.90 \\
1.00 \\
1.00 \\
1.10 \\
1.00 \\
1.00\end{array}$ \\
\hline
\end{tabular}

FIGURE 7.10

Results of measurements obtained from corrosion-casts of vessels which had been anastomosed in the young growing animal.

\section{DISCUSSION}

The results have shown that the sleeve anastomosis has no detrimental effect on the growth of the rat femoral artery.

Russell et al (162) whose rats were allowed to grow from $150 \mathrm{gm}$ at operation to $550 \mathrm{gm}$ at evaluation, found that the ETEA also allowed normal growth of the vessel. The vessels were reported to have shown no evidence of stenosis at the anastomotic sites and to appear histologically normal except for some neovascularisation around the anastomosis. 
Chapter 7 - Ultrastructure

In view of Russel's work I am unable to show that there is any growth advantage in performing the EIEA in growing vessels. I shall aim at performing a controlled comparative study in young animals to determine if there is any advantage for growth of the vessel when the EIEA is used.

$-==<<000>>==-$ 


\section{CHAPTER VIII \\ DISCUSSION}

"The society which scorns excellence in plumbing because plumbing is a humble activity and tolerates shoddiness in philosophy because it is an exalted activity will have neither good plumbing nor good philosophy. Neither its pipes nor its theories will hold water."

John W. Gardner (1974)

\section{HISTORICAL ASPECTS}

The desire to perform a vascular anastomosis without penetrating the endothelium and without exposing suture material to the bloodstream motivated Murphy (135) to employ the sleeve principle.

Carrel's aim initially was to avoid exposing suture material or needle-damaged intima to the blood stream, but he was later influenced by the work of Julius Dorfler (57), who in 1899 showed that these "misdemeanours" could be conducted with impunity when sterilised silk sutures were used. According to Guthrie (71), he and Carrel used Dorfler's methods successfully from 1905 onwards. Despite the availability of the knowledge that vessels could be successfully sutured in this simple way, there were still surgeons, such as Dorrance $(56)$ in 1906 , who sought methods by which they could anastomose vessels, while ensuring clear intima-to-intima contact, with no damaged areas or sutures exposed to the blood stream. 
I am left to wonder whether the efforts to present unspoilt intima to the blood stream could have resulted in methods, which would have been the norm today, had it not been for the findings of Dorfler and their successful application by a surgeon with the standing of Alexis Carrel.

Is it not possible that the original premises of the early vascular surgeons were correct and that the route opened by Dorfler was merely expedient at that time?

The presence of suture material within the lumen of the vessel made little discernable difference to the outcome in large vessels, and it soon had Carrel's stamp of approval which legitimised it for seven decades. With the passage of time suture materials developed considerably. Those materials which were inert and strong and smooth, and thus less likely to have an effect on the blood stream, were eagerly adopted by vascular surgeons for use in anastomoses. These materiais, such as nylon, were made ever thinner and more delicate to accomodate the need for joining very small vessels while placing the materials within the lumina of those vessels. The thrust of development was thus aimed at making suture materials which could with impunity continue to be placed within the lumina of smaller and smaller vessels.

\section{INTIMAL DAMAGE \& EXPOSURE OF SUTURES TO BLOOD STREAM IN SMALL VESSELS}

It is my contention that needle damage to the intima and exposed suture material are suboptimal conditions in vascular anastomoses. Their presence in large vessels is of little consequence, but as vessel diameters get smaller optimisation becomes more critical, to the extent that no less than perfect conditions can be tolerated in very small vessels.

The development of very small sutures has scaled down the technical deficiency in the standard anastomosis of vessels of around $1 \mathrm{~mm}$ in diameter, but in even smaller vessels it has been demonstrated by Huang et al that the success of the anastomosis depends critically upon the thickness of the suture employed (85). Acland has demonstrated the effects of various mechanical insults upon microvessels and concluded that the situation most frequently giving rise to thrombus formation was the placement of an intraluminal suture (8). Pagnanelli et al showed by means of SEM the damage caused by the passage of needles through the vascular endothelium of small blood vessels (149). 


\section{INTUSSUSCEPTION}

The methods which succeeded in creating anastomoses without intraluminal foreign material have all been:

1. methods which employed external mechanical devices, some of which are very modern, such as the Vicryl couplers being developed by Ethicon Inc. [ETHICON, INC., 1979, Somerville, New Jersey, U.S.A.], and others much older $(138,139)$; or

2. methods in which the feeding vessel was placed within the receiving vessel $(135,154,86,112,131,133)$; or

3. a combination of these two concepts as in the method of Payr (Fig 1.2) and those modifications of his method used by Blakemore [reported by Hess (81)] and Kamada and Calne (97).

The new sleeve anastomosis belongs to the second group. in this group there is always a small "step" of non-endothelial tissue at the cut edge of the FV exposed to the blood stream, but there are no large masses of foreign material in the vicinity of the anastomosis as in the ather methods. The position of the step is such that the main thrust of the blood stream should skip over the non-endothelial surface. Lauritzen points out that in the ETEA, any small area of the RV's cut edge which is not flush with that of the FV may be facing the full thrust of the blood stream and it may thus be more likely to initiate thrombus formation (see Fig 8.1). 


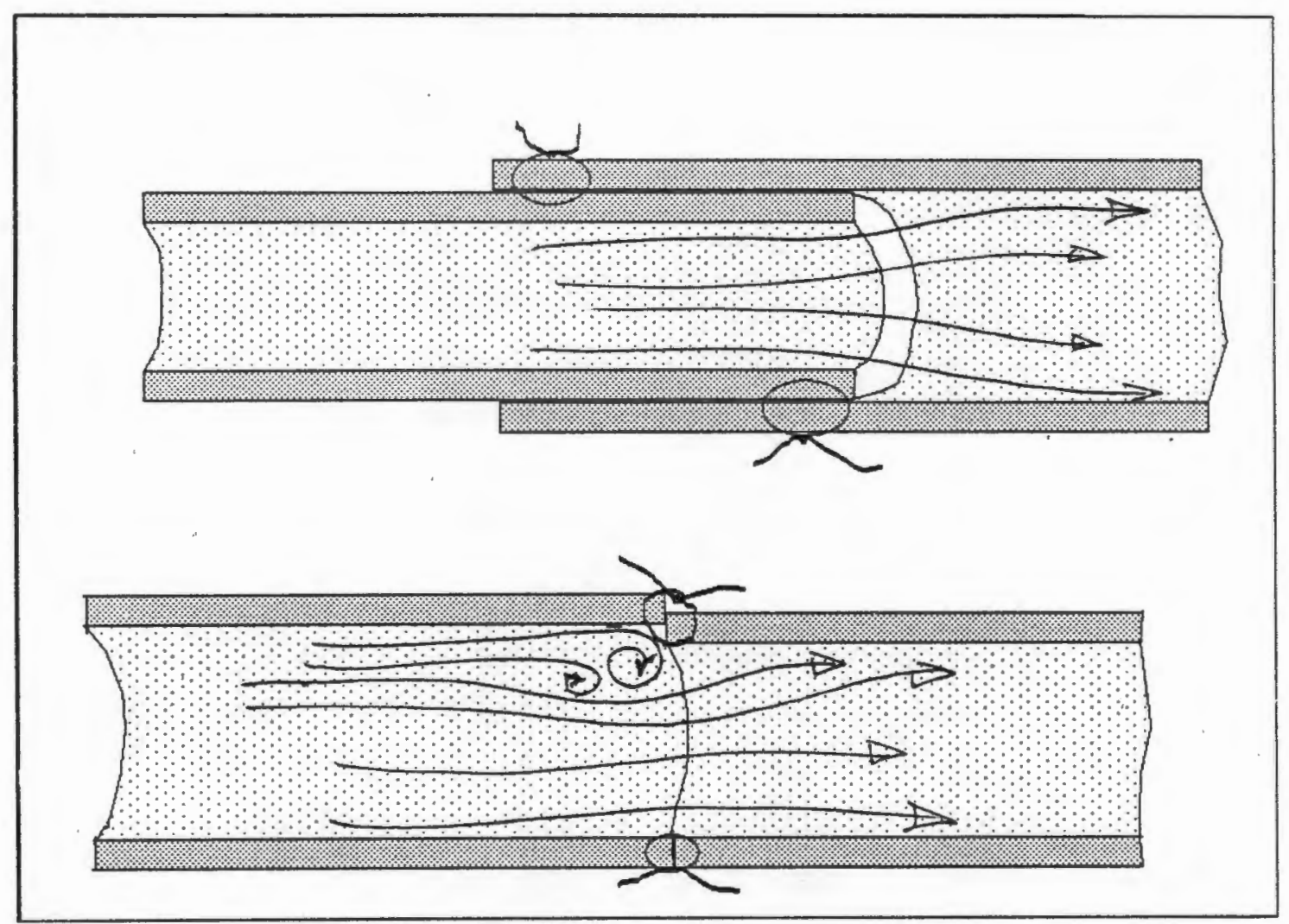

FIGURE 8.1

The "step" in the sleeve anastomosis compared to that which may occur in the ETEA.

\section{MECHANICAL ASPECTS}

\section{STENOSIS}

A degree of physical stenosis is present in most vessels which have been anastomosed by a sleeve method. The degree of stenosis varies with the adequacy of preparation of the vessels for anastomosis; both over-dilation and insufficient dilation of the vessels are likely to result in stenosis. Excessive dilation may cause damage which may lead to stenosis through scarring and cicatrisation of the vessel wall. This danger of course also applies to standard ETEAs. These also frequently have an irregular luminal surface which may be demonstrated by 
corrosion casts. I believe that those irregularities set up eddy currents which have a similar rheological effect to that of the physical stenosis in the sleeve specimens.

\section{FLOW}

The mild stenosis seen to follow the EIEA after good technical preparation of the vessel stumps permits flow, which is not statistically different from that associated with the ETEA, through the reconstituted vessel. In my study there was a statistical difference between the flow through anastomosed and virgin vessels, irrespective of the type of anastomosis used.

\section{SHORTENING}

Telescoping of vessel stumps shortens the vessel by an amount equal to the length of the overlap. This amount of shortening, equal to 1-2 vessel-diameters, is easily tolerated by any vessel which has been divided and directly reconstituted. In situations where vessel-length is critical or inadequate, due to tissue loss or debridement, the sleeve anastomosis should not be used except in association with an interposition graft.

\section{TENSILE STRENGTH}

If early postoperative movement is to be encouraged, the surgeon would like to be assured that the anastomosis has sufficient tensile strength to survive such movements. Previous studies have demonstrated a relative weakness of the sleeve anastomosis in the early postoperative period, but testing was not done under dynamic flow conditions. My measurements, performed while the vessel was normally pressurised from within, showed that it was equal to the ETEA in tensile strength. 


\section{BIOLOGICAL ASPECTS}

\section{HEALING}

Healing of the vesse! occurs at the same pace as that for the ETEA, but there are some significant differences. The sleeve method seldom gives rise to necrosis of the media of the FV, a condition frequently seen in ETEAs. There is also less intimal hyperplasia associated with the sleeve anastomosis than with the ETEA. Krag et al (100) found that there was always evidence of necrosis in the walls of vessels which had been anastomosed by means of the ETEA and that this necrosis might involve the entire area which had been clamped off during the anastomosis. They also found that the sleeve anastomosis was more successful than other methods in vessels which had previously been irradiated with standard therapeutic doses of radiation. This finding may be of interest to surgeons who perform reconstructive surgery after ablative surgery and radiotherapy for malignant neoplastic disease.

is it possible that the vessel wall necrosis is related to operating time? (Blood supply to the wall of the vessel is prevented by the clamp during this time.) If so, the decreased operating time required for the sleeve anastomosis may be of significance, not only to the surgeon but also to the wellbeing of the vessel. I have previously asked myself if necrosis of the media of the RV was not perhaps necessary for the dilation of the EIEA overlap area by the $3^{\text {rd }}$ postoperative day. If so, it may be necessary to ensure that necrosis of the RV media occurs.

\section{ANEURYSMS}

The aneurysms associated with ETEAs $(129,78)$ may be a direct consequence of the mural necrosis mentioned above. Their absence in association with the sleeve anastomosis supports the operating time argument.

The practical value of the absence of aneurysms associated with the sleeve anastomosis $(108,111,113)$ is that aneurysms are extremely undesirable in confined spaces such as found in the finger. Their presence in such areas may even cause damage to other structures (73). 


\section{VESSEL GROWTH}

A limited study has shown that there was negligible impediment of vessel growth where the sleeve anastomosis was used in the vessels of growing animals. In theory too it should be well suited to allowing growth, due to the use of fewer sutures, less mural necrosis and consequently less scarring.

\section{SURGICALASPECTS}

"In clinical microsurgery it is desirable to use as few sutures as possible when performing a microvascular anastomosis to save time and to minimise trauma to the vessels......A reduction in the number of sutures can decrease operating time and reduce the likelihood of technical error - especially in small vessels."

Harris, Finseth \& Buncke $(76,77)$

\section{GENERAL}

\section{TIMESAVING}

All the surgeons who have studied sleeve anastomoses have agreed that these can be done more quickly than ETEAs $(112,108,65,131,133,87,142,178)$. In my comparative study the new sleeve anastomosis could be consistently performed within approximately one third of the time required for an ETEA.

The benefits of this timesaving to the surgeon, the patient, the anaesthetist and the theatre staff are obvious, and the physiological benefits to the reconstituted vessels have been mentioned above. 


\section{SIMPLICITY}

The simplicity of the new EIEA has been demonstrated by the rapid acceptance and use of the method by novice microsurgeons, and more subtly, by the high degree of negative covariability between vessel diameter and operating time in very small vessels (see Chap 5).

\section{CLINICAL MICROSURGERY}

\section{APPLICATIONS}

I had not, until recently, completed my experimental investigations of the new sleeve anastomosis and have therefore not had the opportunity of using it in many clinical situations. There have, however, been a few situations in which I have considered application of the new anastomosis to be to the patient's advantage and I have thus proceeded with clinical use of the technique.

\section{Case 1}

The right forearm and hand of a man had sustained a severe crush injury in a mining accident. Initial examination of the injured limb revealed that the hand was attatched to the dorsal surface of the forearm by a $2 \mathrm{~cm}$-wide strip of skin and fascia, which had been rendered non-viable by crush injury. There was no blood supply to the hand.

The distal ends of the forearm bones appeared to be crushed flat and were contaminated with grease and gravel. There were severe crush and laceration injuries to the soft tissues of the forearm. The patient was resuscitated and taken to the operating theatre with the intention of debriding the forearm and completing the amputation.

At operation it was noted that the crushing force had been applied to a narrow area, sparing the distal $2 \mathrm{~cm}$ of the forearm bones. The crushed skin bridge between the hand and the forearm was divided and the hand placed on sterile instant coldpacks (MEDAC (Pty.) Ltd., Bree St., Cape Town 8000 , Rep. of South Africa). The radius and ulna were each shortened by $6 \mathrm{~cm}$ and the soft tissues debrided in both stumps. 
The bones were fixed by means of A.O. dynamic compression plates (SYNTHES Pty. Ltd.) and the arterial supply was re-established via two $8 \mathrm{~cm}$-long reversed saphenous vein grafts to the radial and ulnar arteries. The FV-to-graft anastomoses were done by the new sleeve method and the graft-to-RV by the "Posterior wall first" method of Harris, Finseth and Buncke (76). Six cutaneous veins were anastomosed directly by the new sleeve method. The median and ulnar nerves were repaired, as were all the extensor tendons and the deep flexor tendons.

The hand remained well perfused during the post-operative period and the surface wounds healed well.

\section{Case 2}
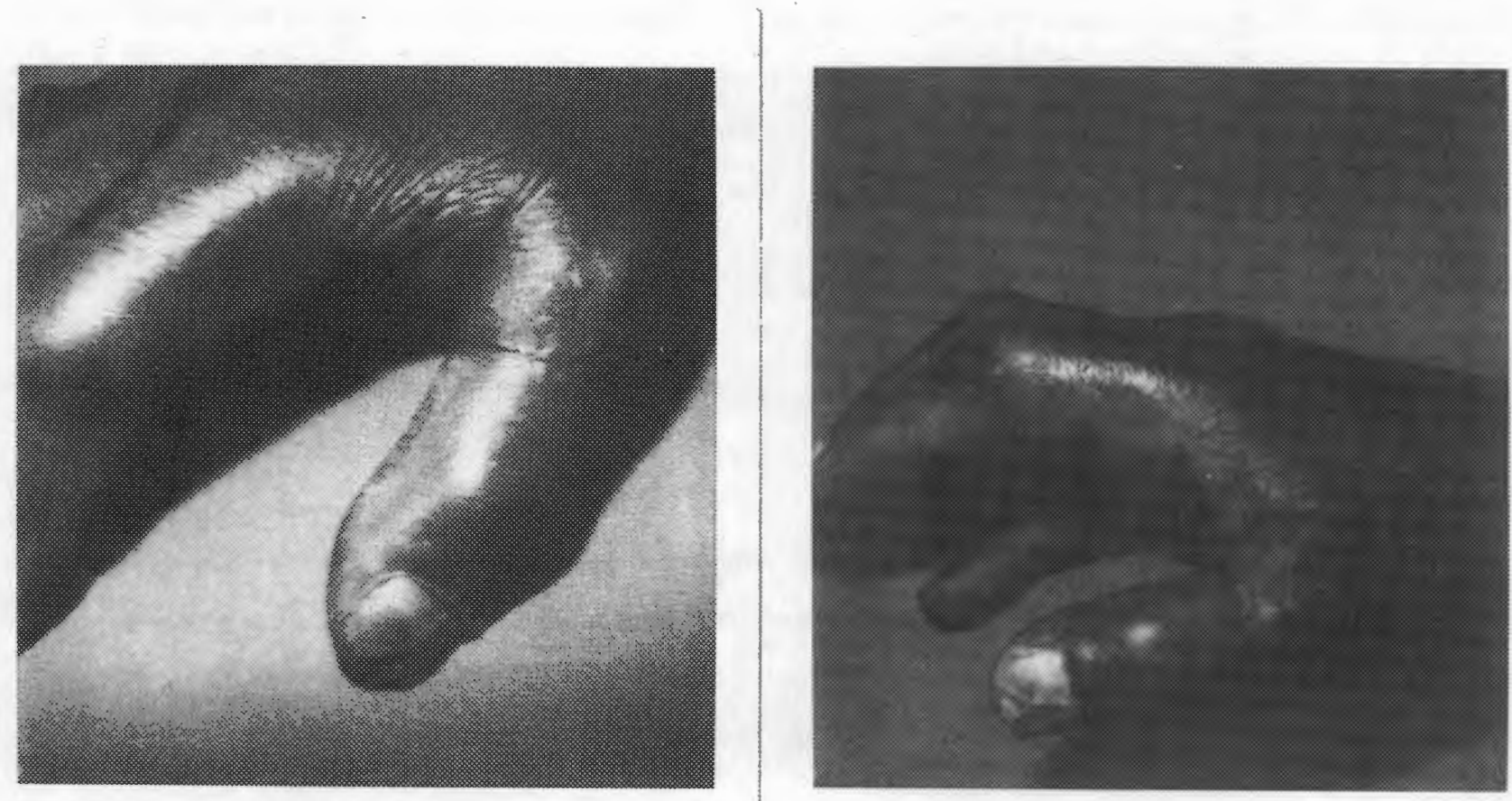

\section{FIGURE 8.2}

The replanted thumb described in Case 2 


\section{Case 2}

A 42yr-old man cut off his right thumb $1 \mathrm{~cm}$ distal to the metacarpo-phalangeal joint in a bandsaw accident. The thumb was brought to hospital in a clean plastic bag by the patient who requested replantation.

At operation, which started 1 hours after the accident, very little soft tissue debridement was required. The proximal phalanx of the thumb was fixed by means of interosseous wiring. Two arteries and 3 veins were repaired by means of the new sleeve anastomosis. The EPL, EPB, FPL and APL tendons and digital nerves were repaired primarily and a silastic rod was placed in the FPL tendon sheath next to the tendon to maintain a passage in case a secondary grafting of that tendon should be required. A wedge- shaped portion of the thumbnail was removed and the nail-bed cleaned hourly with a heparinised swab as recommended by Buncke. This was continued for the first two postoperative days.

The thumb made an uneventful recovery and the silastic rod was removed after 6 weeks as it was not needed.

\section{EXPECTATIONS}

The new sleeve anastomosis has worked well in very small vessels and in growing vessels. I expect that it should therefore be of value to microsurgeons who are involved in paediatric replantation and transplantation surgery. Surgeons dealing with lymphatic reconstrucion may also find application for the technique and further research in this direction should be carried out.

\section{EXPERIMENTAL MICROSURGERY}

\section{APPLICATIONS}

Most of the evaluation of the new EIEA has been done in the vessels of small animals such as the rat. In these vessels it has functioned well with associated time-saving and simplicity of 
execution. These qualities make it a highly desirable technique in experimental transplantation and replantation procedures.

The microsurgery laboratory of the University of Cape Town's Department of Surgery devotes much of its time and effort to the establishment of models for the study of hepatic transplantation in the rat. Mr G.H.C. Engelbrecht and his laboratory assistants have developed a method for the removal and orthotopic replantation of the rat liver to serve as a control for homozygous heterogeneic orthotopic transplantation of the liver. Orthotopic replantation of the rat liver has not been previously described, and is much more difficult than transplantation due to the limited vessel lengths available. The method has been further refined in this laboratory by including an anastomosis of the hepatic artery by means of the new sleeve anastomosis. In Hepatic transplantation where extra vessel length is available as for the cuffing techniques of Kamada and Caine (97), all the anastomoses may be carried out rapidly and without the presence of bulky foreign bodies by using the new EIEA.

I believe that it may be important in the establishment of surgical models to match operating time to the life rhythms and metabolic rates of the animals concerned. Unfortunately there is usually an inverse relationship between the size of the animal and its metabolic rate. The degree of difficulty of the operation is also inversely related to the size of the animal, and transplantation operations on small animals therefore probably take much longer than they should. Any method which can reduce operating time and simplify procedures should therefore contribute to the improvement of the physiological validity of experimental models.

Other sieeve methods have been successfully used in various experimental surgical procedures $(41,65,142)$ and the new method should further contribute to success in those areas.

\section{EXPECTATIONS}

Limited preliminary studies have shown that the new sleeve anastomosis may be employed successfully in larger vessels. It may therefore be of special benefit in the reconstitution of larger vessels in experimental transplantation studies. The hepatic artery of the pig is of specific interest to our experimental surgery programme and I hope that this vessel, which at $2.5 \mathrm{~mm}$ diameter is smaller than general surgeons would like it to be but is larger than a microvessel, may prove amenable to sleeve anastomosis. 


\section{FUTURE RESEARCH}

\section{VASCULAR SURGERY}

\section{LARGER VESSELS}

Development and further modification of the new sleeve anastomosis in larger vessels may lead to the solution that Murphy, Carrel and other pioneers were originally seeking. There have been major improvements in surgical materials and instrumentation since their time and a new solution is therefore not impossible.

The problem of stenosis due to thicker walls in larger vessels may possibly be overcome by different preparation of the vessel ends e.g. by oblique cuts (see Fig 8.3).

\section{ASSOCIATIONS WITH ADHESIVES}

Sugiura et al (179) have combined the use of the EIEA and fibrin glue to develop a new method for the anastomosis of interposition vein grafts.

The sleeve anastomosis is particularly suited to the association with exogenous adhesives, because of the intermural seal established by the blood pressure. This seal should prevent contact between the exogenous adhesive and the blood stream and thus prevent the possibility of a disastrous intravascular clotting cascade triggered by the adhesive. Externally the adhesive may provide support by sticking the vessel stumps together and by sticking the stumps in their telescoped position to the surrounding tissues. This may help to maintain their positions relative to each other. 


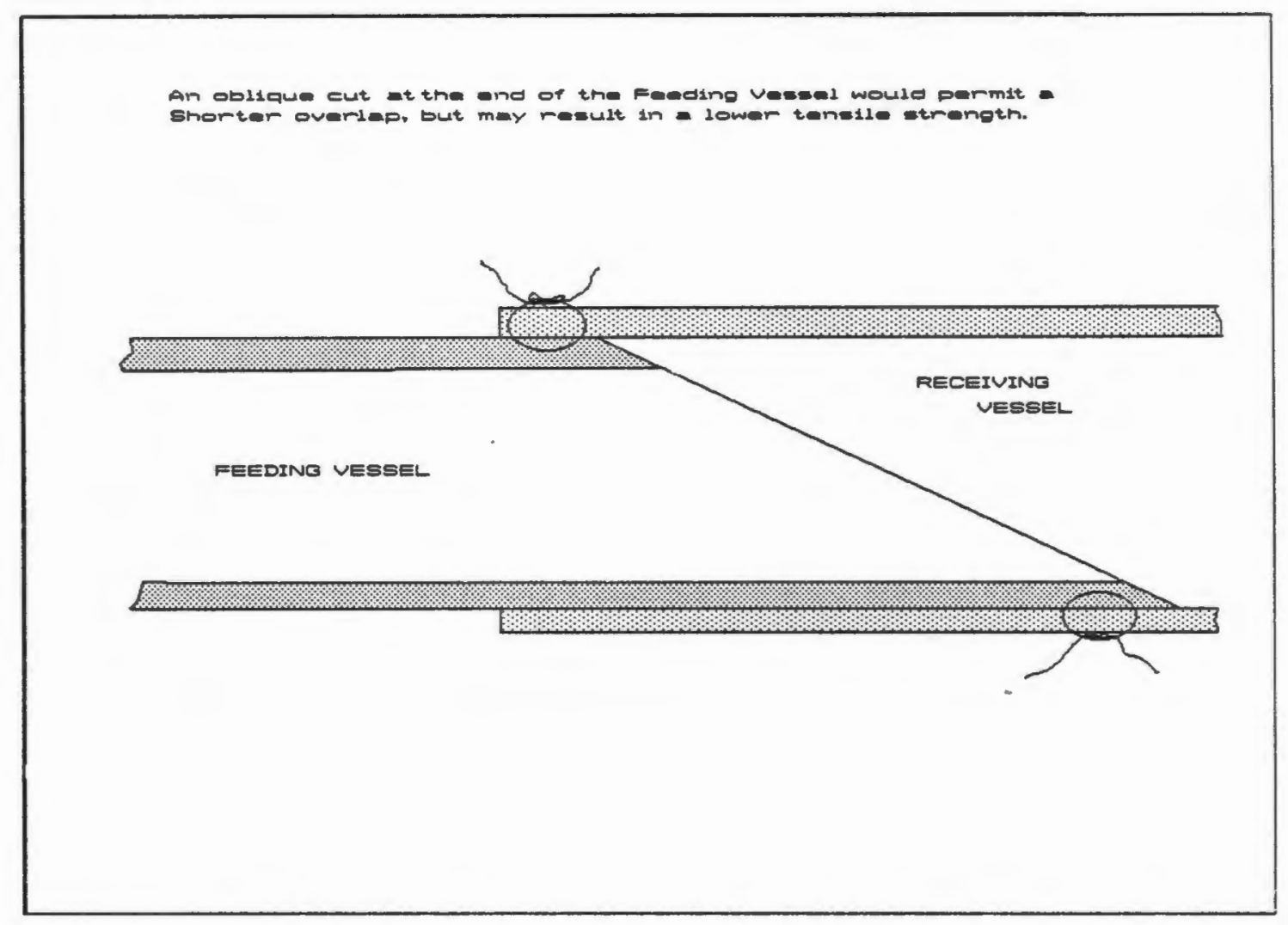

FIGURE 8.3

Proposed oblique cut of FV end for larger vessels.

\section{IMMEDIATE REMOVAL OF SUTURES}

I have found it possible, in reconstituting the rat femoral artery, to perform a sutureless sleeve anastomosis without using any glue other than the endogenous fibrin. This was done by omitting heparin from the irrigating solution, leaving the sutures untied at the end of the anastomosis and simply holding them under tension to keep the vessels telescoped while removing the vascular clamps from the artery and applying them to the sutures. The sutures were then held in position, while applying mild tension, to hold the vessel ends in the correct position for 15-20 minutes. Thereafter the sutures were released and removed. To my surprise the vessels did not become dislodged and retained the appearance of a normal sleeve anastomosis except for the absence of sutures.

It would probably be safer to use a heparinised irrigating solution, but it may then be necessary to use exogenous fibrin glue to create such sutureless anastomoses. The new sleeve technique 
should provide a reliable method for achieving relatively atraumatic telescoping in the execution of a new generation of anastomoses which do not have any significant areas of scarring in their walls.

\section{SMALLER VESSELS "MINI-MICRO"}

The anastomosis of very small vessels must have numerous possible applications in experimental surgery where small animals are used to study physiological changes associated with surgical procedures such as transplantation. We have already seen such an application in the anastomosis of the rat's hepatic artery. This could previously only be done by dissecting the vessel back to the aorta and then performing an end to side anastomosis of the donor's aorta to that of the recipient to re-establish flow through the donor's hepatic artery. This procedure was, of course, totally impossible in orthotopic replantation of the liver.

The feasibility of anastomosing very small vessels may open the routes for experimental investigation of the effects of transplantation of other organs in small animals, or it may improve the accuracy of existing investigative procedures.

In clinical microsurgery the anastomosis of very small vessels may become useful in paediatric microvascular surgery. It is therefore neccessary to examine the use of the sleeve anastomosis in very small vessels in larger animals.

\section{LYMPHATIC SURGERY}

The sleeve anastomosis may simplify lymphatic reconstruction where vessels are usually both small and thin-walled. The pressure and flow characteristics in lymphatic vessels must differ greatly from those in blood vessels and research in the use of the anastomosis in this field is therefore essential. 


\title{
CONCLUSION
}

\author{
In microarterial anastomosis the surgeon should \\ endeavour to achieve an ultimately leak-free anastomosis \\ with as few sutures as possible to avoid medial necrosis \\ and arterial occlusion.
}

Hayhurst \& O'Brien (78)

\begin{abstract}
The new sleeve anastomosis requires only two sutures. It has proved to be simple to perform and has consequently been enthusiastically accepted by both experienced and novice microsurgeons. It is based upon the concept which yielded the first recorded successes in reconstructive vascular surgery.

In the hands of an experienced microsurgeon there is no significant difference in the patency rate between this procedure and that of the ETEA, but novice microsurgeons obtain better patency rates with the new sleeve anastomosis.
\end{abstract}

Microsurgeons, over a wide range of expertise, have uniformly and significantly reduced their operating time by using the new sleeve anastomosis, and they take between one third and one half of the time they had previously required.

The new anastomosis is based on sound mechanical principles and has been shown to match the ETEA in both its tensile strength and the flow permitted to pass through it.

As previous exponents of the sleeve principle have pointed out, the sleeve anastomosis has the following disadvantages:

1. Extra vessel length is required for the overlap.

2. It cannot be used to unite two vessels when there is a large difference between their respective diameters.

3. The technique cannot be used where end-to-side anastomoses are required. 
The new sleeve anastomosis described in this thesis represents a development and advance on previous sleeve methods. The asymmetrical suture placement brings about a decrease in technical complications and an increase in stability of the anastomosis. These factors lead to consistent time-saving and reliability of the anastomosis.

Since Lauritzen is the recognised authority on the sleeve anastomosis in microvascular surgery, I shall let him have the last word (see Appendix D):

"I think your method is the ultimate sophistication of the sleeve anastomosis, I would use it myself."

Claes Lauritzen (1987) 


\section{APPENDIX A \\ THE POLISHED-NEEDLE CANNULA}

\section{INTRODUCTION}

In microvascular surgery the need for a fine-bore irrigating cannula frequently arises. The cannula is usually used to direct a stream of irrigant into the lumen of a vessel, in order to remove potentially thrombogenic blood products before making an anastomosis. Very delicate hypodermic needles can be obtained with relative ease and they have the inherent advantage that they can be connected directly to a syringe or an intravenous fluid line filled with irrigating solution.

Surgeons usually feel better about using hypodermic needles as irrigating cannulae if they have been blunted by being rubbed point first on a piece of sandpaper or other abrasive material. Blunt needles are also commercially available in a number of gauges. The reason for blunting needles or purchasing blunt needles has never been clear to me. Students of microvascular surgery are invariably advised not to enter the lumen of a vessel with anything other than the very tips of their fine forceps or with the polished tips of vessel-stretching forceps. It would therefore seem prudent to leave the needle sharp, thus providing a deterrent against the occasional irresponsible urge to take the cannula a little closer to the vessel in order to remove the last stubborn fragment of blood clot from the lumen. 


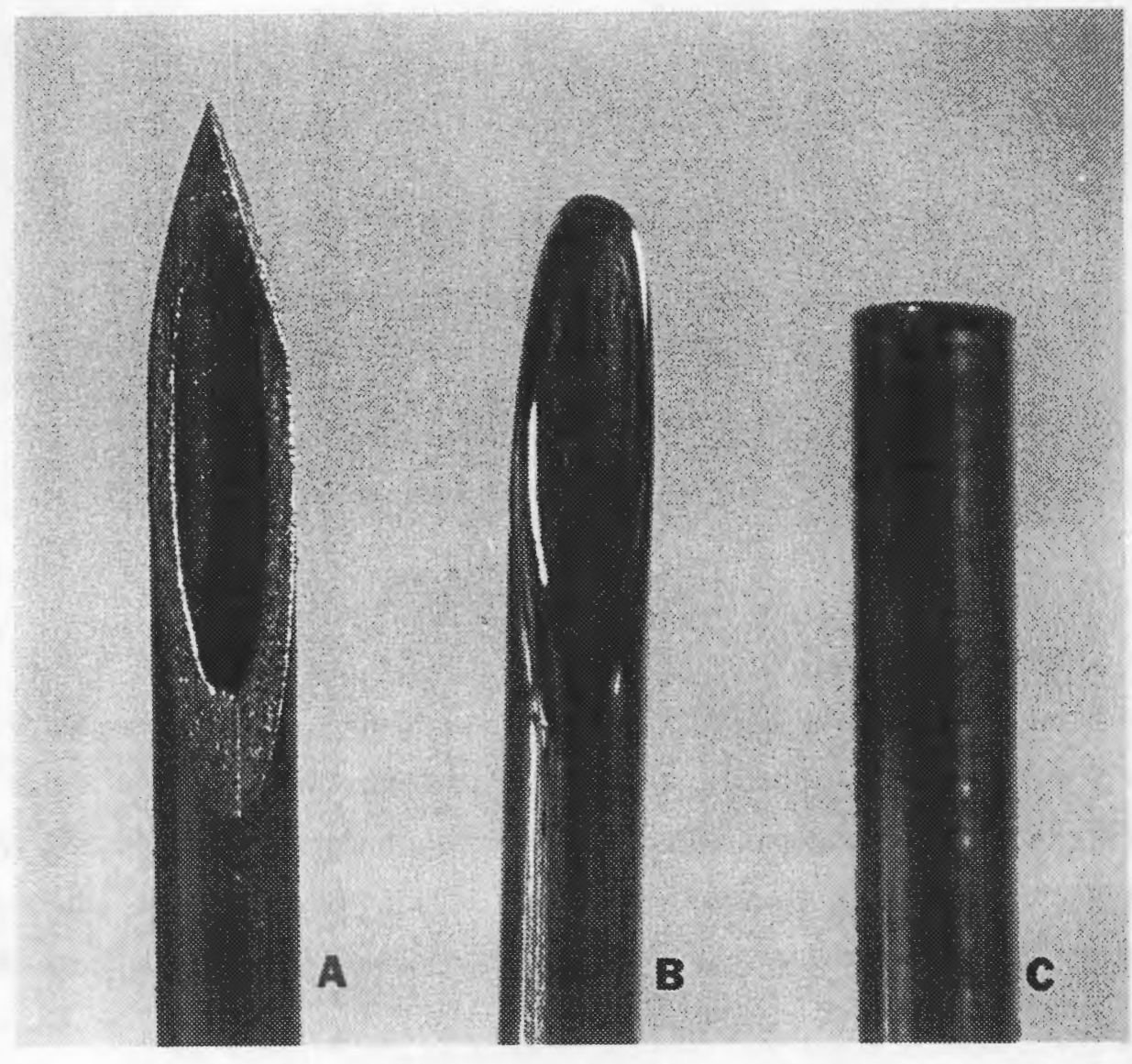

FIGURE A.1

A standard Hypodermic needle (A), a hand polished blunt needle (B) and a commercially available blunt needle (C)

Both hand-blunted hypodermic needles and commercially avallable blunt needles have, under high magnification, surfaces and edges which have a very rough appearance (Fig A.1). It is conceivable that such needles may cause covert intimal damage by abrasion if they are brought into contact with the intraluminal surface while being used to irrigate vessel ends prior to anastomosis.

It was argued that, if vessel-stretching forceps, which require insertion into the lumen and forceful contact with the intimal surface, could be used with impunity (8), a correctly polished cannula could be employed within the lumen without subjecting the intima to injury. The task of removing every sharp edge and rough abrasive surface from a normal hypodermic needle was therefore examined, with the hope of producing a perfectly smooth and atraumatic microcannula.

In order to achieve this objective, it is necessary to identify every sharp edge and rough surface and then to polish each of them without simultaneously producing another sharp edge. The 
production of such a cannula is a simple matter which is well within the ability of any microsurgeon. The polished needles or cannulae proved to be adaptable to a number of uses in microsurgery, i.e. as intraluminal irrigators, as hydrodissectors within vascular sheaths, as backwall protectors and counterpressure devices during anastomosis and as hydrostatic vessel dilators.

\section{MATERIALS AND METHODS}

Only four devices are required, viz. a sheet of fine emery paper 600-800 grit, a motor-tool (such as the Dremel tool with a small buffing wheel attachment), a stick of jeweler's rouge and a set of operating loupes or a laboratory operating microscope.

The same set of steps is used for needles of all sizes. We use a set consisting of the following gauges: $30,25,21,18$. ) (Fig A.2)

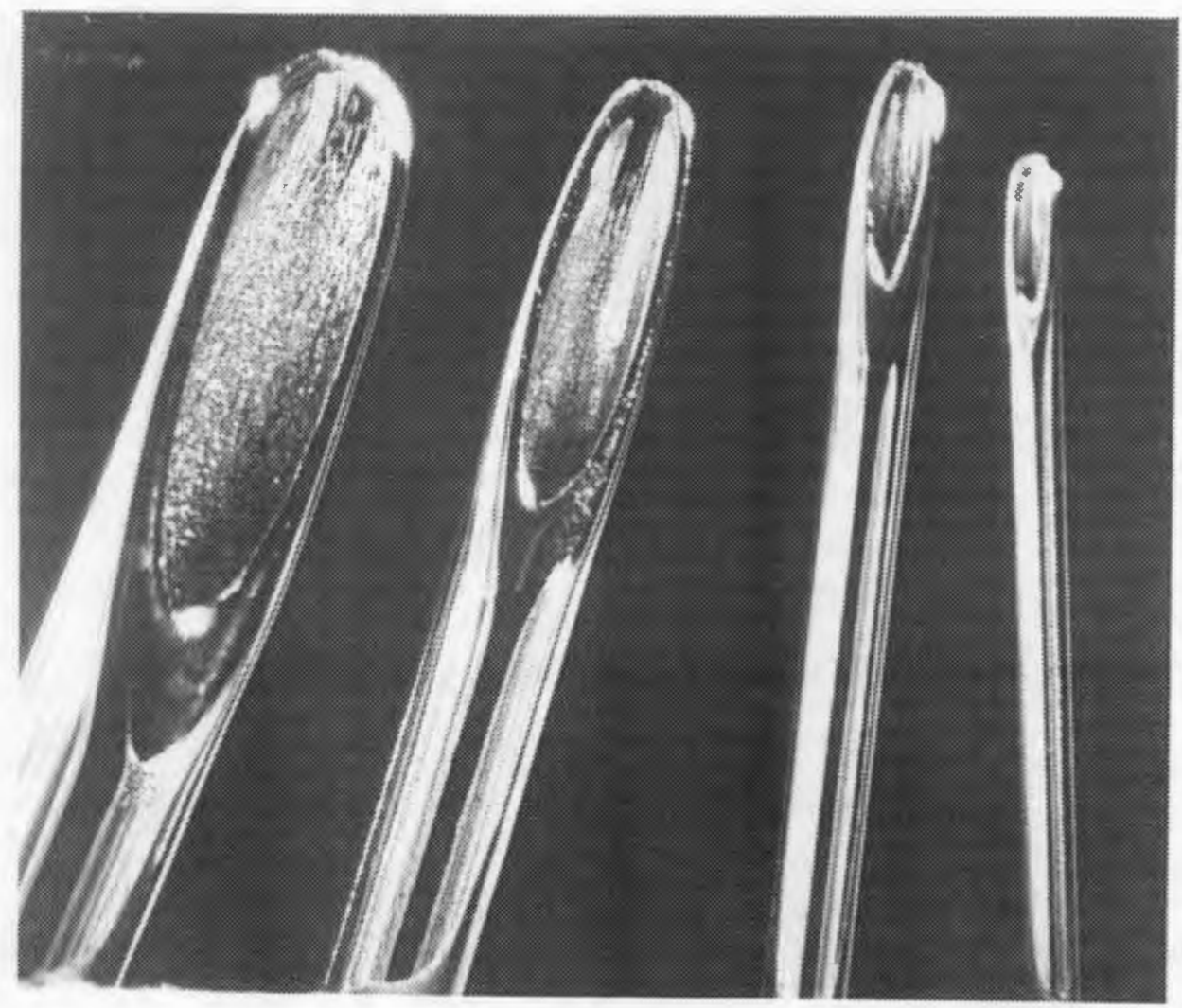

FIGURE A.2

The set of polished needles. 


\section{POLISHING METHOD}

\section{STEP ONE:}

With the needle held at approximately 30 degrees to the horizontal, bevel side upwards, the point of the needle is stroked back and forth in a $3 / 8$ circle arc over the surface of the emery paper which is resting flat on a firm surface.

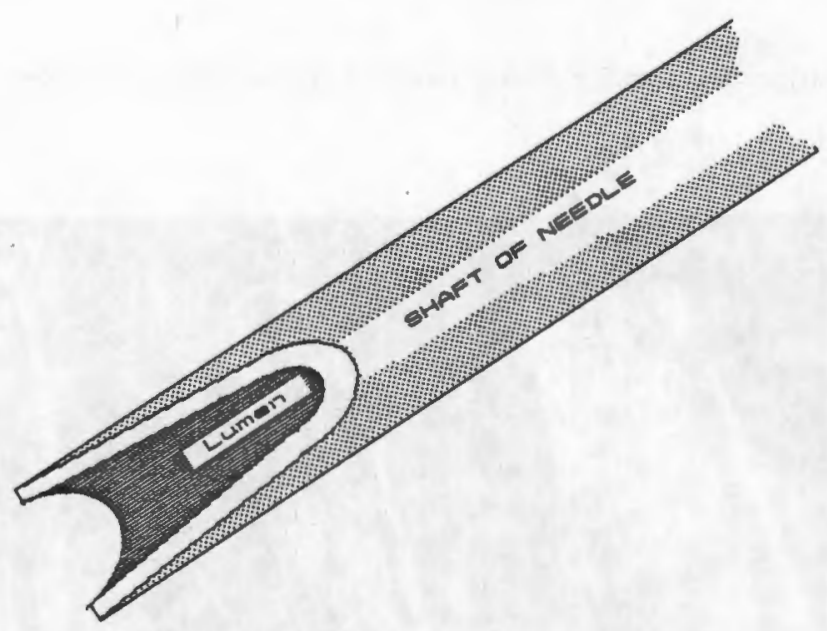

FIGURE A.3

The two-pronged fork which results from grinding too much away.

This produces the rounded point which is also beveled slightly towards the lumen of the needle. The procedure should be performed under magnification while carefully checking that only the point of the needle is ground away. If taken too far the resultant "two-pronged fork" should be discarded and a fresh start made. (Fig A.3) 

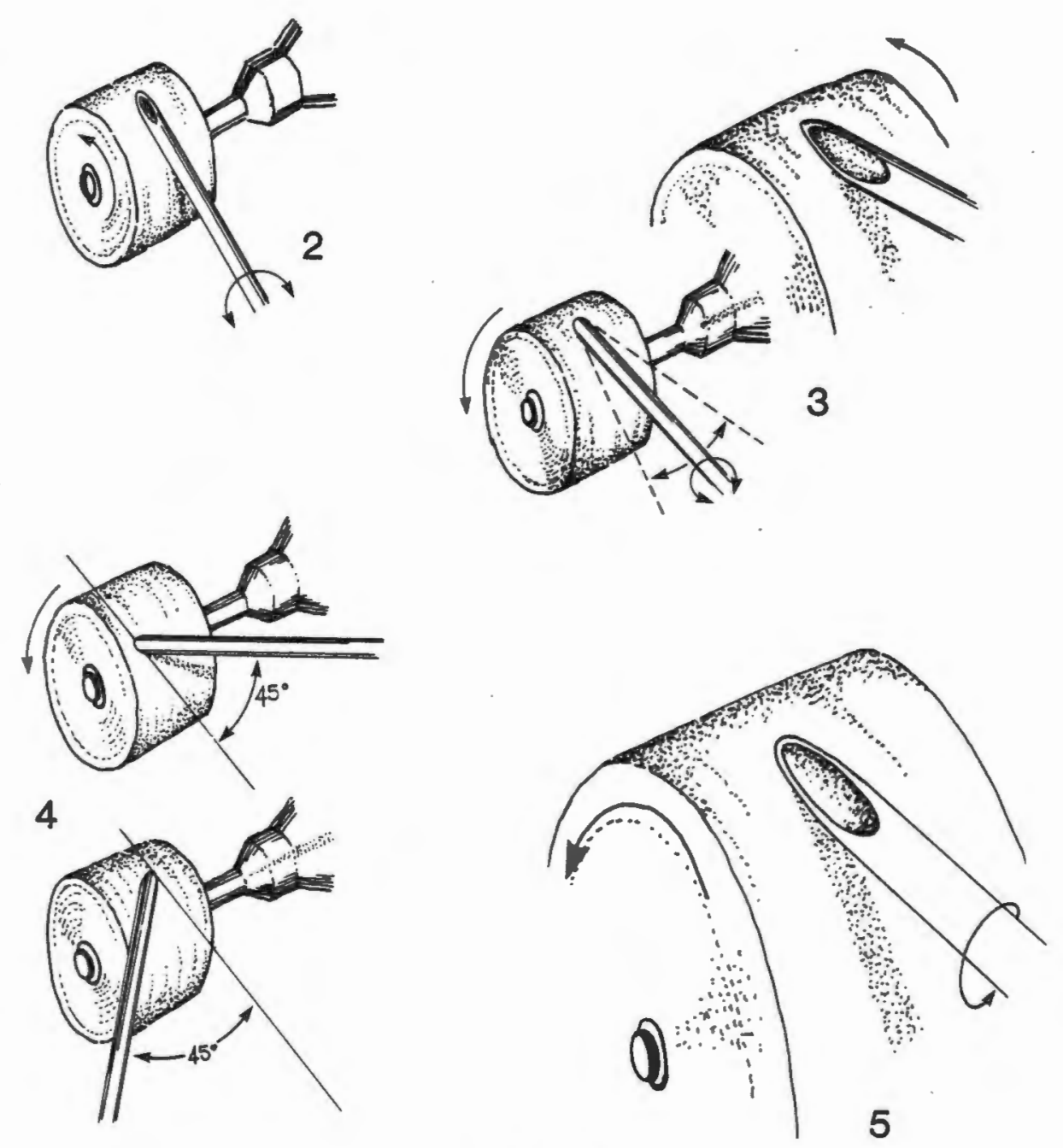

FIGURE A.4

Steps of the polishing process.

\section{STEP TWO:}

The area which has been roughly ground away is now polished by matching the longitudinal alignment of the needle with the buffing wheel's rotational direction and performing gentle toand-fro axial rotation of the needle while in contact with the buff. (Fig A.4.1) 


\section{STEP THREE:}

The outside edges of the bevel are rounded and polished with the same longitudinal position and a similar motion to that in step two. During this step the inside of the rounded front edge of the needle will also receive some polishing. Care should be taken to keep the back edge of the bevel away from the wheel, since this edge would otherwise be sharpened (Fig A.4.2).

\section{STEP FOUR:}

The inside of the bevel needs to be smoothed. This is done by holding the long axis of the needle at approximately 45 degrees to the rotational direction of the buffing wheel. The needie is rotated around its long axis in such a way that the front edge is touching the wheel and the back edge is away from the wheel. The needle is then switched to the opposite hand and held at 45 degrees to the direction of the wheel to polish the other inner edge of the bevel (Fig A.4.3).

\section{STEP FIVE:}

The whole shaft surface is polished by longitudinal alignment with the wheel and full rotation of the needle while still taking care that the back edge of the bevel is not sharpened (Fig A.4.4).

\section{STEP SIX:}

The needle is gently palpated for sharp edges. (For very fine needles this is best done with your tongue). If no sharp edges are felt, the needle is gently washed in a soapy solution to remove all traces of rouge and is then irrigated through with distilled water by means of a syringe. The needle can be packed and sterilised in the usual manner. It is best to use needles with metal hubs, as repeated sterilising will damage plastic ones. After use needles should be cleaned (preferably in an ultrasonic cleaning bath) and then rinsed in distilled water to remove solutes which may crystallise in the lumen during sterilisation or storage. Once learned, the whole procedure for polishing a needle is quick and simple and takes no more than five minutes per needle. 


\section{USING THE POLISHED-NEEDLE CANNULA}

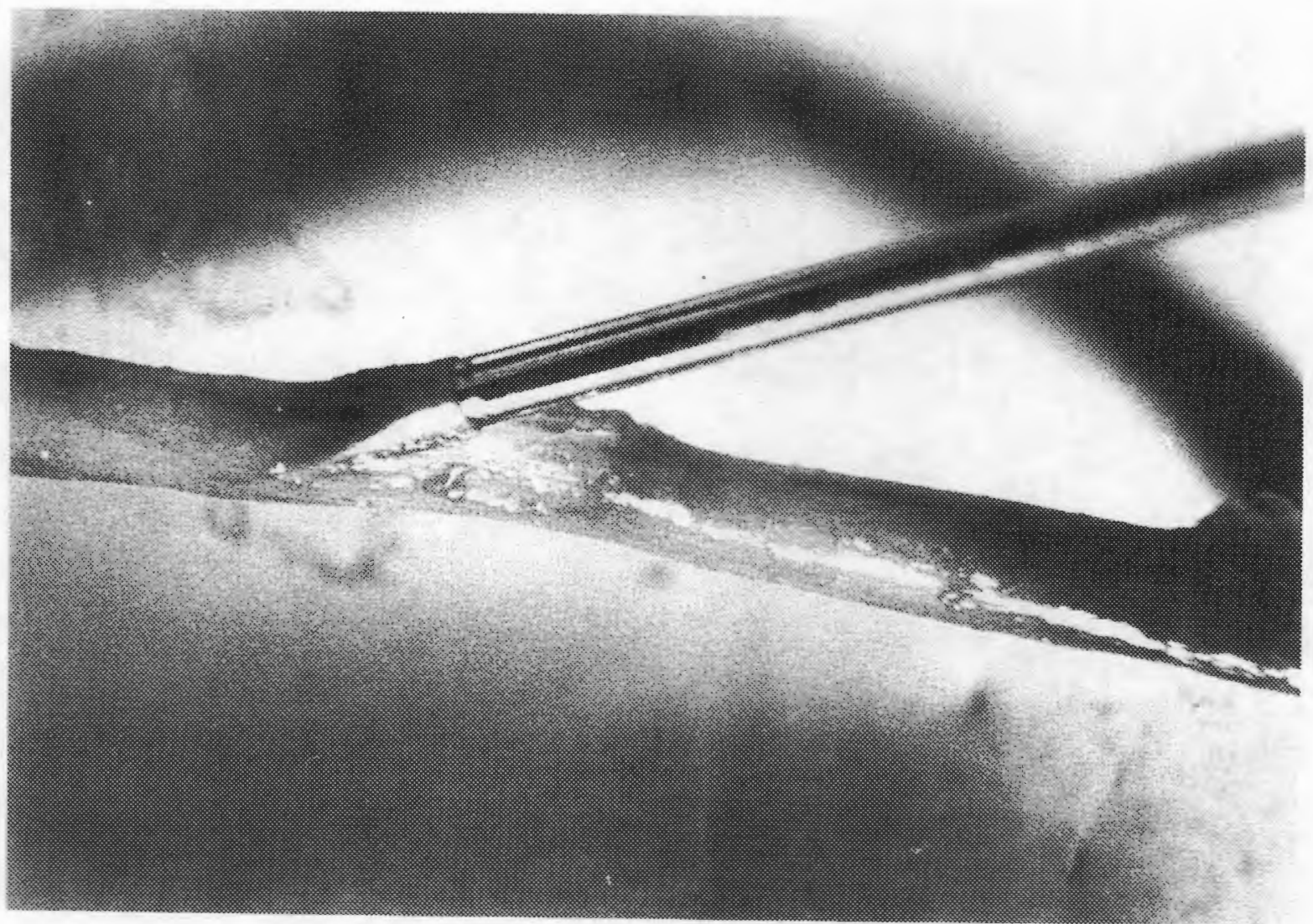

FIGURE A.5

Introducing the polished needle cannula into the vessel to flush out blood.

\section{IRRIGATION}

The cannula may be bent slightly near the tip by using a rubber-covered haemostat. It may then be inserted, without fear of abrasion to the intima, deep into the lumen of a vessel end to flush out any thrombogenic components (Fig A.5).

\section{HYDRODISSECTION}

The cannula may be carefully inserted into perivascular fibrous sheaths, where it tends to glide between intact vessel walls without damaging them. When the tip of the cannula has nudged its 
way to a position between the vessels, fluid is injected, thus separating the vessels from each other and from the sheath and greatly facilitating sharp dissection (Fig A.6).

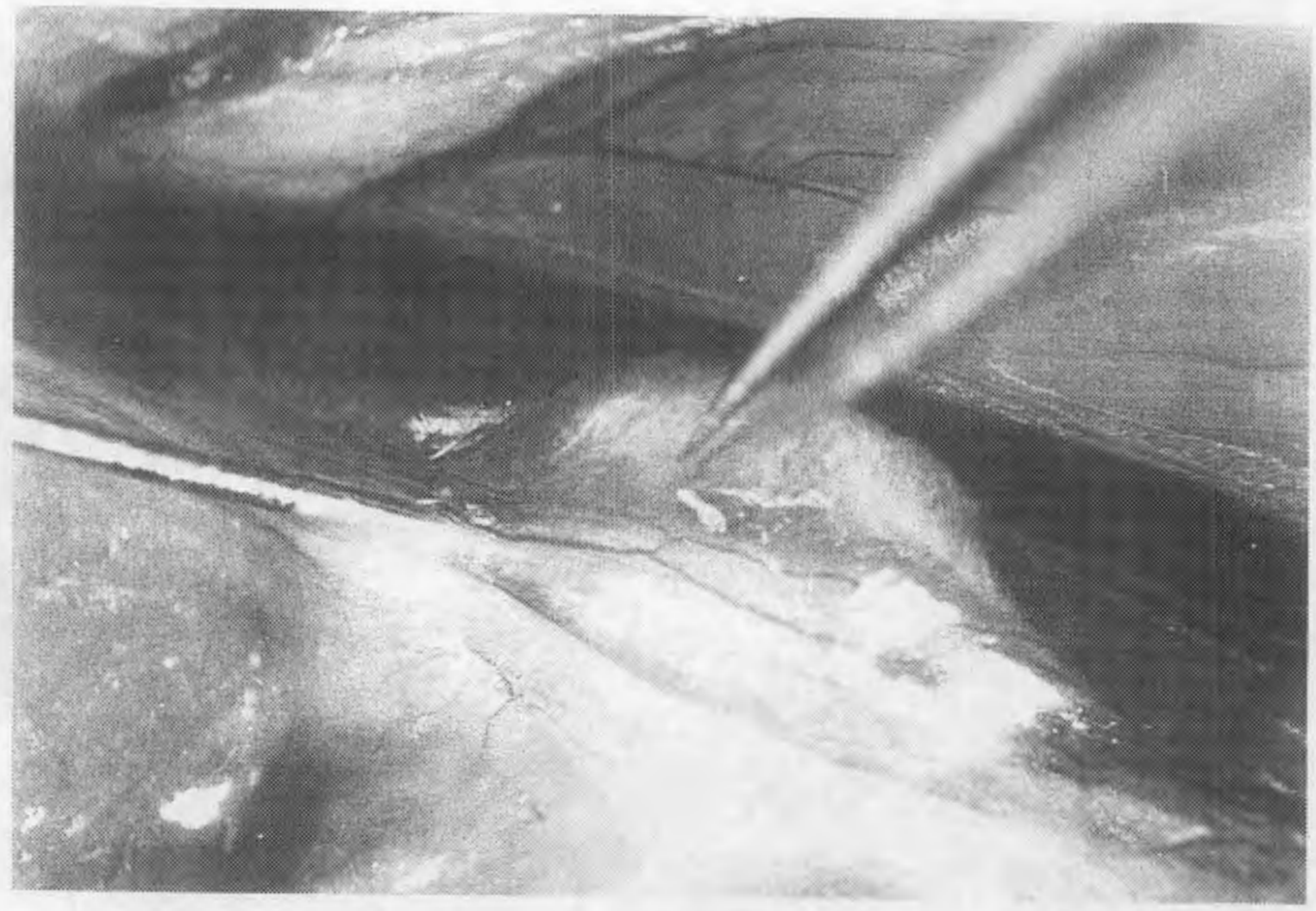

FIGURE A.6

Hydro-dissection by means of the polished needle cannula.

This process is much simplified by the use of the polished cannula. The use of normal injection needles often leads to trauma of a vessel wall by the sharp needlepoint. If required, vasodilators may be added to the dissecting fluid, thereby aiding visualisation of the vessels and their separation from surrounding tissues. 


\section{COUNTERPAESSURE \& BACKWALL PROTECTION}

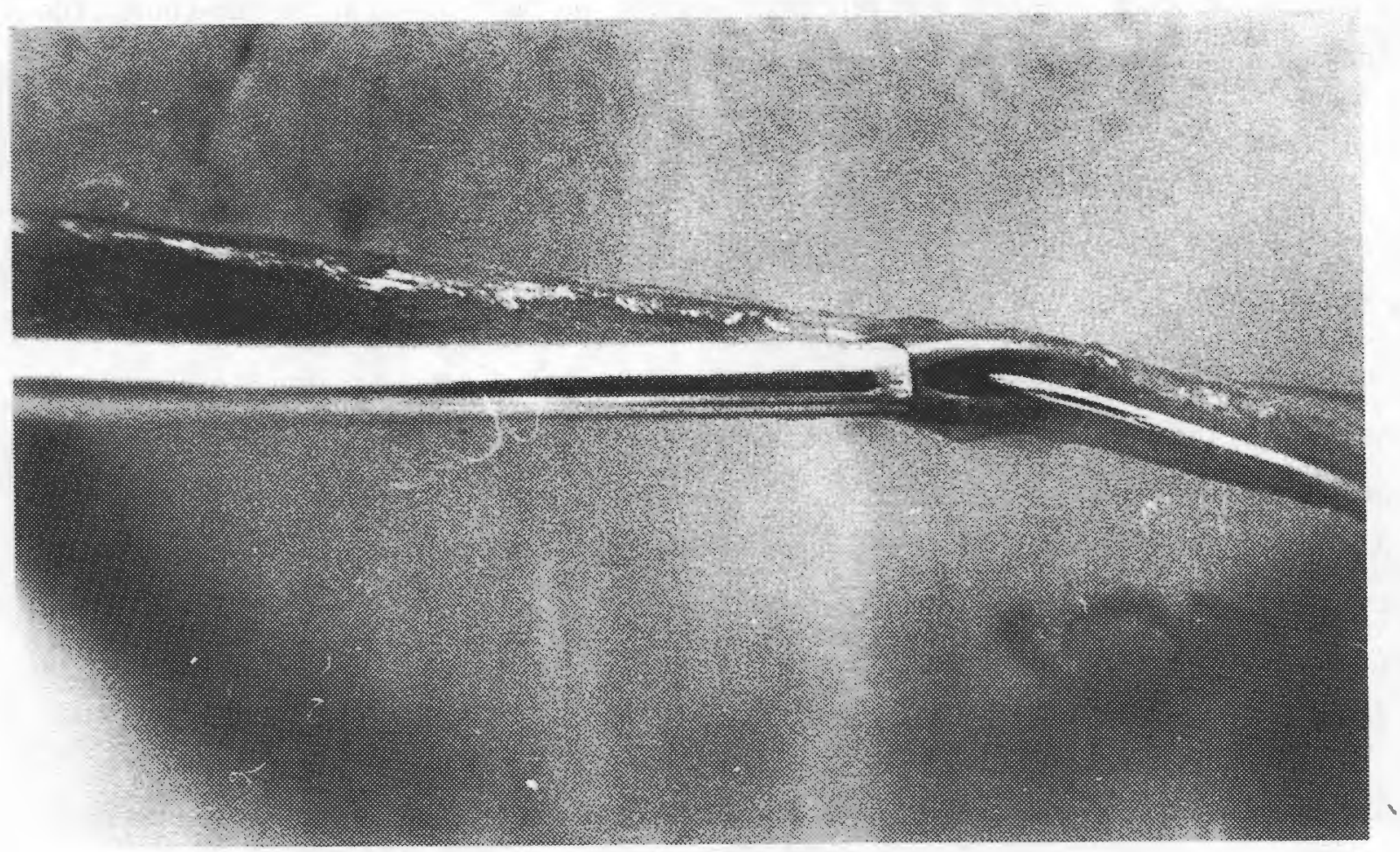

FIGURE A.7

Counterpressure device and backwall protector.

Using a lcc syringe as a handle. the cannula may be inserted into the lumen of the blood vessel. The suturing needle is then passed through the wall of the vessel and into the hollow of the lumen of the cannula which thus acts as a counterpressure (in a way similar to Acland's counterpressure loop (5)). At the same time it "barricades" the backwall with the posterior wall of the cannula (Fig A.7).

\section{HYDRODILATION}

When placing the last one or two sutures in an anastomosis the surgeon may be at greatest risk of picking up the backwall. By passing the cannula through the last small opening (without fear of trauma) and injecting a physiological solution such as Ringer's lactate into the vessel before placing the last suture, the vessel is held open by the fluid, while the backwall is pushed out of the way of the suturing needle. The injection of an irrigating fluid at this stage has the further 
advantages that the lumen will be washed clear of heparin, while air bubbles and any residual debris, which could impede flow in very small vessels, will be removed at the same time. The final suture can then be placed with less risk of including the backwall.

\section{DISCUSSION}

I have used these instruments for the past three years in clinical and experimental microsurgical operations. They have made an enormous difference to the ease with which dissection is performed within neurovascular bundles, by enhancing both the speed and the accuracy of the dissections. Students of basic microsurgical techniques in our laboratory have enthusiastically adopted the needle as an essential part of their dissecting equipment and we see far fewer vascular disruptions during dissection by members of this group than was previously our experience.

As a counterpressure device and backwall protector during suture placement the needle plays a vital part in the suturing technique for the new sleeve anastomosis. When a suture needle is passed from outside through the vessel wall and into the lumen of the cannula, the polished rim of the beveled area provides an encircling, atraumatic counterpressure and, furthermore, the fact that the suture needle ends up within the hollow shaft of the cannula means that the backwall of the vessel is totally protected while the needle is being brought out of the vascular lumen.

We are certain that the abovementioned uses may be applied to many procedures in other surgical fields. We hope that this extremely simple instrument may greatly benefit the surgical practice of those who take the trouble to make it or to obtain it. (Commercial prototypes of this cannula have been produced by SSC, Schaffhausen, Switzerland.) 


\section{APPENDIX B \\ THE PRODUCTION AND USE OF CORROSION CASTS \\ FOR THE EVALUATION OF \\ EXPERIMENTAL MICROVASCULAR ANASTOMOSES}

\section{INTRODUCTION}

The use of corrosion casts in the study of microvascular anastomoses has previously been proposed and used in conjunction with Scanning Electron Microscopy by P. A. van Gelder (196). We were not aware of this proposal when we set out to study microvascular anastomoses by means of corrosion endocasts and we consequently had to employ techniques of our own. The results we obtained by this practical and direct method of evaluation allow us to support the proposal made by Van Gelder.

The practice of injecting solidifying compounds into hollow organs and then removing the tissues by chemical corrosion has been established for more than 300 years. Narat et al (140) have researched the literature and found publications dating from that of Swammerdam (circa 1790), who is recognised as having introduced the method to medical science. Their research of the literature revealed a "density" of publications in the latter part of the 19th century. A wide variety of casting materials, including wax, metal alloys, celluloid, guncotton and latex was used with varying degrees of success. Judging by the amount of effort, patience and perseverance required, the production of corrosion casts in earlier times must have been a character-building experience.

The use of plastics or resins in a liquid monomer form, which can be induced to form solid polymers by the addition of catalysts and promotors, was originated by Batson (16) and has been further developed as more modern materials have become available. The desirable characteristics of a corrosion compound, as set out by Batson, are the following:

1. It should have a minimum of shrinkage. 
2. It should be usable at room temperature.

3. It should be readily injectable by several means.

The availability of new materials has greatly improved the quality of the specimens which can be obtained, but we have found that there has been little discussion of the exact techniques used for the introduction of the material into the tissue specimen. We suspect that there has been little change in these techniques since the earliest times.

We encountered a number of problems, for which we developed our own solutions, in the production of endocasts of rat blood vessels to study microsurgical anastomoses. In this paper we intend to describe a technique which can be easily duplicated and may be of assistance to those who wish to study experimental microvascular anastomoses by means of corrosion endocasts.

\section{MATERIALS AND METHODS}

The material used in the formation of endocasts was Batson's \#17 Anatomical Corrosion Compound (Polysciences Inc.). This material is supplied in a kit form and contains a monomer base solution, a catalyst, a promoter and coloured pigments.

The anatomical models we wished to study were the blood vessels of adult Sprague-Dawley rats $(300 \mathrm{~g}-400 \mathrm{~g})$. In most cases we were interested in the femoral arteries only, but we also had cases in which we wished to study both larger and smaller arteries and veins.

\section{TECHNIQUE FOR ENDOCAST PRODUCTION}

We initially aimed at producing endocasts of the desired anatomical region only, by, for example, dissecting free and cannulating the infrarenal aorta in order to fill the femoral arteries. This, however, proved to be tedious, not conducive to standardisation of technique when other vessels were to be studied and also not always technically simple. The dissection of the aorta may result in nuisance tears in the inferior vena cava, thus complicating the procedure tremendously. 
As a result of the difficulties mentioned above, we decided to develop a standardised method for the production of endocasts of blood vessels which are situated anywhere in the rat. The procedure may be carried out with the aid of $4 X$ loupes or under the lowest power of the operating microscope. An irrigating mixture of normal saline $1 \mathrm{~L}+$ Heparin 1000 units $+20 \mathrm{ml}$ of Xylocaine $2 \%$ is prepared and warmed to 40 degrees $C$. When the irrigating fluid is ready and warm, it is connected to an intravenous administration set and suspended from a drip stand. The intravenous set is run through and connected to a 19-gauge butterfly needle which has been specially polished (cf. pol ished-needle cannula).

The animal is deeply anaesthetised with Ketamine $10 \mathrm{mg} / 100 \mathrm{~g}$ + Acepromazine $5 \mathrm{mg} / 100 \mathrm{~g}$ (body weight) given by intraperitoneal injection, followed by a further injection of Nembutal $0,1 \mathrm{ml} / 100 \mathrm{~g}$ by the intraperitoneal route when the animal is asleep.

The chest is shaved clean and the animal is placed on its back and held in position with soft, loose restraints to ensure that venous return is not occluded by them. An elasticised retractor is placed in readiness on either side of the chest.

Other pieces of equipment which should be ready to hand are:-

1. A small needle-holder or a mosquito forceps armed with $5 / 0$ Dexon on a cardiac needle or a round-bodied needle.

2. A small container of cyano-acrylate adhesive ("crazy-glue" or "superglue") which can be obtained at most hardware stores. The glue works best if an injection needle is fitted over the nozzle, but a fine nozzle is adequate.

3. A few $4 \times 4$ gauze swabs or sponges.

4. A pair of fine Metzenbaum scissors and a pair of heavy-duty straight scissors.

5. A Brown-Adson forceps is useful, but not essential.

6. Suction apparatus or any other effluent-collecting system will facilitate the procedure.

The first part of the procedure should be accomplished fairly rapidly and it is best to check that all the equipment is ready to hand and that the animal is adequately anaesthetised before proceeding. 
When all is ready, the surgeon makes a longitudinal skin incision over the full length of the sternum. The pair of Metzenbaum scissors is used to dissect into the thoracic cavity on the left side of the xiphisternum, while taking care to stay above the diaphragm and not to enter the peritoneal cavity. The pair of straight scissors is used to cut through the length of the sternum. The thoracic cavity is opened and held open by the two lateral elasticised retractors.

The pericardium is opened with the Metzenbaum scissors and the rounded point of the scissors is used to lift the heart forward. The heart is carefully observed while it is beating and the apex of the left ventricle is identified. The Brown-Adson or any similar forceps is then used in the left hand to grab the myocardium at a point roughly $2 \mathrm{~mm}$ to the right of the apex.

By applying slight traction and $20^{\circ}$ to $30^{\circ}$ of anti-clockwise rotation to the heart (Fig B.1), one can clearly see the outline of the pulsating left ventricle. The butterfly needle is positioned 1 $\mathrm{mm}$ to the left of the apex and advanced longitudinally into the left ventricle, while aiming at the left ear of the animal. As soon as the needle is in the ventricle the blood will be seen "flashing" into the tube of the butterfly needle with each pulsation. It is unwise to advance the needle much further after seeing the "flashing", as the interventricular septum may be penetrated.

After making sure that there is no tension on the tube of the butterfly needle, the surgeon, who is still grasping the heart with the left-hand forceps, releases the grip of the right hand on the butterly needle and allows a few drops of irrigant to run into the left ventricle. This usually results in pallor of the heart as clear fluid enters the coronary arteries and is soon followed by cardiac arrest and slight dilation of the left ventricle.

The irrigant is closed off, the needle holder is taken in the right hand and the suture needle is passed through the myocardium from left to right around the butterfly needle (Fig B.2). The lefthand forceps is carefully released and a double-throw knot is made but not pulled tight. A drop of "superglue" is applied to the junction of the myocardium and the butterfly needle and the suture is tied down into the drop of glue. 


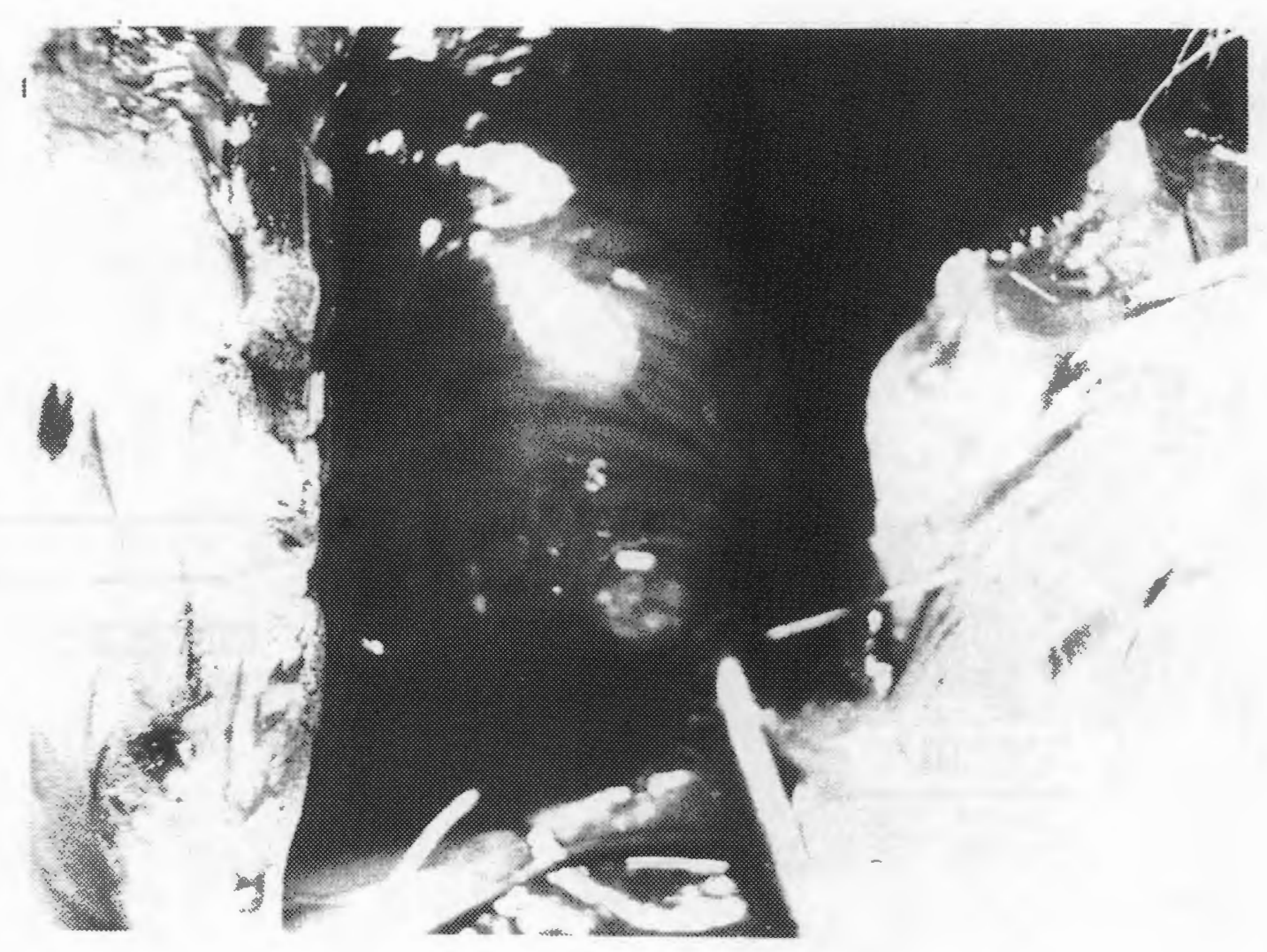

FIGURE B.2

The suture-needle is passed through the myocardium from left to right around the butterfly needle.

The ends of the suture are passed around the needle in opposite directions and tied again on top of the needle and in the drop of glue. In this way the needle and the suture are instantly glued together (Fig B.3). The suture passes through the myocardium and the needle is thus very firmly and stably fixed in place. The irrigation line is again cautiously opened while looking for undue dilation of the left ventricle or sudden bulging of the right ventricle, since this would imply penetration of the interventricular septum. 


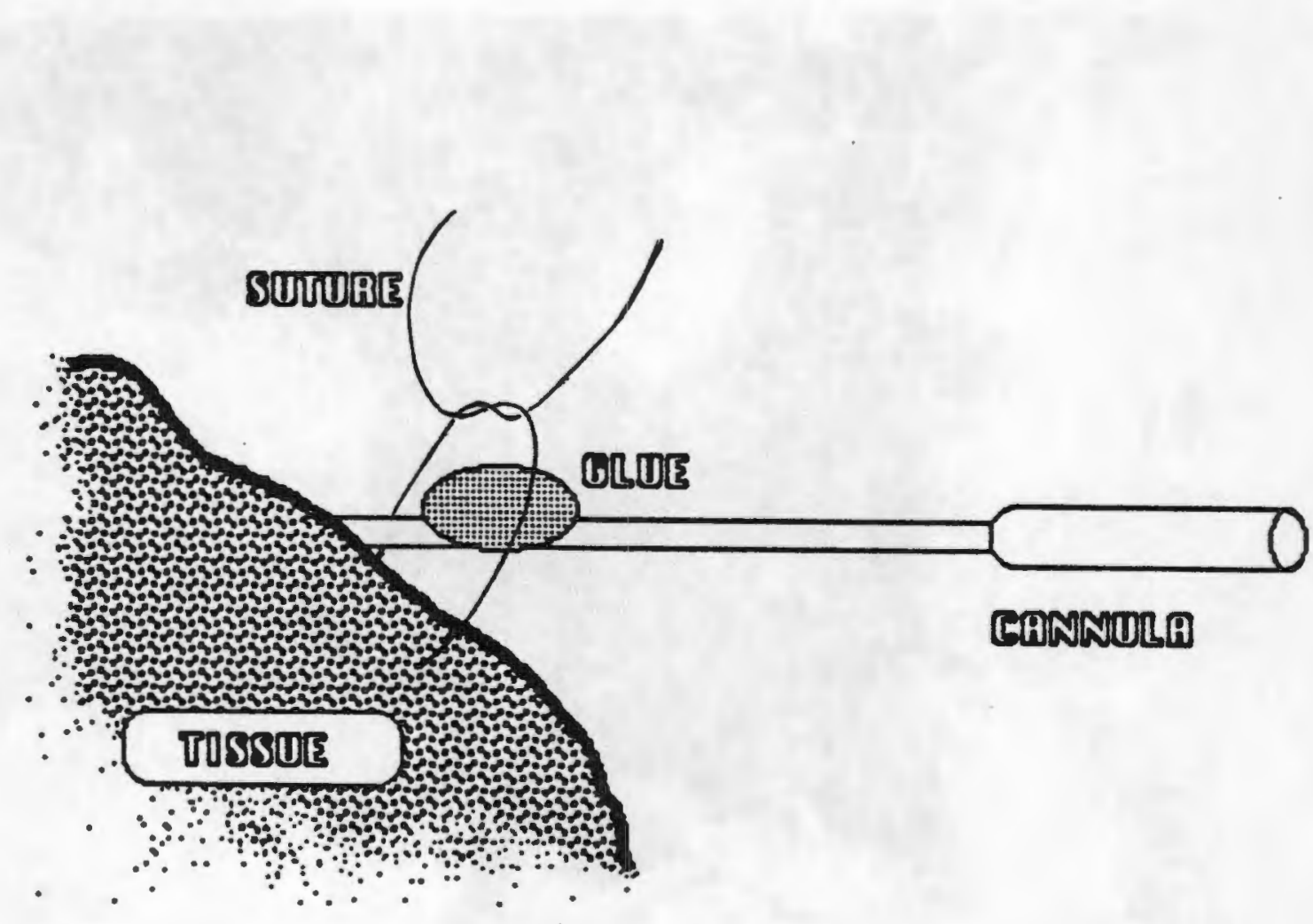

FIGURE B.3

The ends of the suture are passed around the needle in opposite directions and tied again on top of the needle and in the drop of glue.

With the suction apparatus in attendance, the left auricle, which should be starting to dilate, is grasped by means of the Brown-Adson forceps and pulled from its hiding place under the cut edge of the right half of the sternum. The Metzenbaum scissors is used to cut the auricle off and the resulting sudden, severe venous bleeding is removed by the suction apparatus. Having allowed free venous outflow, the irrigant is now set to run as fast as possible until the outflow from the right auricle is completely clear. The animal's eyes, nose and foot pads are checked to see if they are all pale to the point of being off-white in colour. When this point has been reached, the animal is also suitably prepared for other investigations, which will be mentioned in the discussion.

The polymerising resin casting-material should be injected as soon as possible after mixing. It is therefore good practice to check off all the required items against a list before mixing is started. The items required are the following: 
1. Four disposable syringes made of semi-opaque plastic. (The clear plastics tend to react with components of the resin and then to soften). We use TERUMO ${ }^{T M}$ syringes (Terumo Corporation, Tokyo, Japan) and we recommend that one of each of the following sizes be available: $50 \mathrm{cc}, 20 \mathrm{cc}, 5 \mathrm{cc}, 1 \mathrm{cc}$.

2. The resin (at $4^{\circ} \mathrm{C}$ ) already pigmented.

3. The Catalyst.

4. The Promotor.

5. A glass container with a capacity of at least $50 \mathrm{cc}$, for mixing. (The container should be disposable e.g. a pathology specimen bottle.)

6. A few disposable stirring rods.

7. A long, large-bore needle for drawing the mixed compound into the 50cc syringe from the mixing jar and 4 large-bore injection needles.

The resin-casting compound is now prepared by adding catalyst and promotor (ratio: res:cat:prom $=100: 10: 1$ ) and stirring vigorously. We have found that $30 \mathrm{ml}$ of compound is usually more than enough for the arterial system of a large rat and we therefore mix $30 \mathrm{ml}$ of base with $3 \mathrm{ml}$ of catalyst and $0.3 \mathrm{ml}$ of promotor. Great care should be taken not to contaminate the different components of the resin before mixing. We therefore use the $20 \mathrm{cc}$ syringe exclusively for measuring the resin and delivering it to the mixing jar. The 5cc syringe is only used for the catalyst and the 1cc syringe for the promotor. Each syringe is fitted with a large-bore needle which is discarded after the mix. The $20 \mathrm{cc}, 5 \mathrm{cc}$ and $1 \mathrm{cc}$ syringes are kept in separate containers for re-use.

Colouring material should be added to the base at least an hour before mixing to allow complete dispersion of small particles of dye. The compound is kept cold until the time of mixing.

As soon as the compound is homogeneously mixed, the tube of the butterfly needle is clamped off with a haemostat and the IV administration set removed. The compound is drawn up in the 
disposable 50cc syringe and injected into the port of the butterfly needle with slow, steady pressure. Care must be taken that the pressure exerted does not cause any undue bulging of the left ventricle or of the ascending aorta, which should be clearly visible.

If it is deemed necessary, pressure-measuring equipment may be connected to the infusion line, but this point will be dealt with in the discussion. The infusion of compound is continued until the vessels in the toes are seen to fill. When red compound is used in the arteries, the skin begins to take on a natural, healthy appearance. If difficulty is experienced with the detection of these signs, the tips of one or two toes on each paw may be snipped off and the arrival of a telltale red droplet awaited.

The tube of the butterfly set is once again clamped and the syringe removed from the port. A 4 $x 4$ gauze swab is rolled up and placed in the region of the right atrium before relaxing the retractors. This causes some pressure on the atrium, thus preventing further outflow of irrigant and maintaining some back-pressure in the venous system for a short while. The back-pressure sufficiently delays the crossing of the capillary beds by the compound, thus allowing the polymerising process to increase the viscosity. This in turn inhibits free flow through the capillary-bed and thus maintains the volume of the compound remaining in the arterial system.

The injected animal is placed in a bath of ice water $\left(0^{\circ} \mathrm{C}\right)$ to promote slow and even curing of the compound and to conduct away the potentially damaging heat of the exothermic reaction of polymerisation of the resin. We usually keep our specimens in cold water overnight.

If specific vessels are required for study, these may be dissected out within blocks of tissue; otherwise the whole animal may be corroded away. The macerating or corroding fluid we used, was made up of $350 \mathrm{~g}$ of $\mathrm{KOH}$ in $1000 \mathrm{cc}$ of water. The specimen was held down by elastic bands or any other means in a container and covered completely with the macerating solution. The container of plastic or stainless steel is ideally kept in a water bath at $40^{\circ}-50^{\circ} \mathrm{C}$. The macerating solution should occasionally be agitated and may require complete replacement for large specimens.

We found that the removal of the skin of the rat before maceration held advantages in that the process was speeded up considerably. The macerating solution also remained active longer and was more efficient, because of less tissue bulk and less fat in the specimen. The corroded specimen also had a better appearance due to its not being covered in a "haze" of skin capillaries. 
When the specimen appears to be completely cleared of tissue, usually after 24 - 36 hours, it should be carefully washed in warm running water and then allowed to dry or to be blow-dried by compressed air. In many instances a white, patchy film will be seen oyer the vessels. This is the fibrous component of the vessel- walls and it should be removed by placing the specimen in a fresh macerating solution for a further $24-36$ hours. We have found that these fibrous remnants may sometimes be very stubborn but that they can finally be removed by an ultrasonic cleaning bath if all else fails.

\section{VENOUS ENDOCASTS}

The production of whole-body venous endocasts in an animal as small as the rat presents some technical problems. It may be possible to cannulate the very distal veins, but the cannulae would be so fine-bored that the moderately viscous compound would not be able to flow through them quickly enough to fill the venous system before setting or curing. We therefore found it best to do a retrograde filling of the venous system which displays the central system well, but does not fill beyond the inferior superficial epigastric vein because of venous valves. When such a venous endocast is to be made, the auricle is not cut off.

We have tried various methods for cannulating the right atrium, but the results were unreliable owing to the extreme flimsiness of the walls of the large veins and the atrium. We had some success with a method in which the atrium was covered in "superglue" and a piece of supporting soft tissue from the chest wall glued over it, but we finally settled for cannulation of the right ventricle with advance of the cannula to the point where it passed through the tricuspid valve into the right atrium. This cannula is made in exactly the same way as the left ventricular cannula and it is fixed in place in the same way. During the "washing out" procedure it is used as the outflow tract. When corrosion compound is injected through this cannula, the lungs should be packed down with gauze sponges, as some of the material will flow through the pulmonary arteries and cause the lungs to be distended and thus waste corrosion compound. The liver usually takes up a very large portion of the injected compound, thus compensating in volume- requirement for the fact that the peripheral capillaries are not filled from the venous side. Thirty $\mathrm{ml}$ of compound are usually adequate for the venous system of the rat. 
Appendix B - Corrosion Cast Evaluation System

\section{EXAMINATION OF ENDOCASTS}

Endocasts may be used in a number of ways to evaluate the inner surfaces of the vessels which are being investigated. Van Gelder (196) used Scanning Electron Microscopy, but it is also possible to examine specimens and take measurements by light microscopy while using incident light. Direct measurements, using micrometers, may also be possible. We chose to use a photomicrographic method of assessment and measurement. The segments of vessel casts containing the anastomoses were placed in a specially made, axially-rotatable stand (Fig B.4), with a stainless steel rod, $0.9 \mathrm{~mm}$ in diameter, placed parallel to them in all three dimensions. The vessel-cast and standard rod were placed as close as possible to the surface of a plane which lay perpendicular to the optical axis of the microscope.

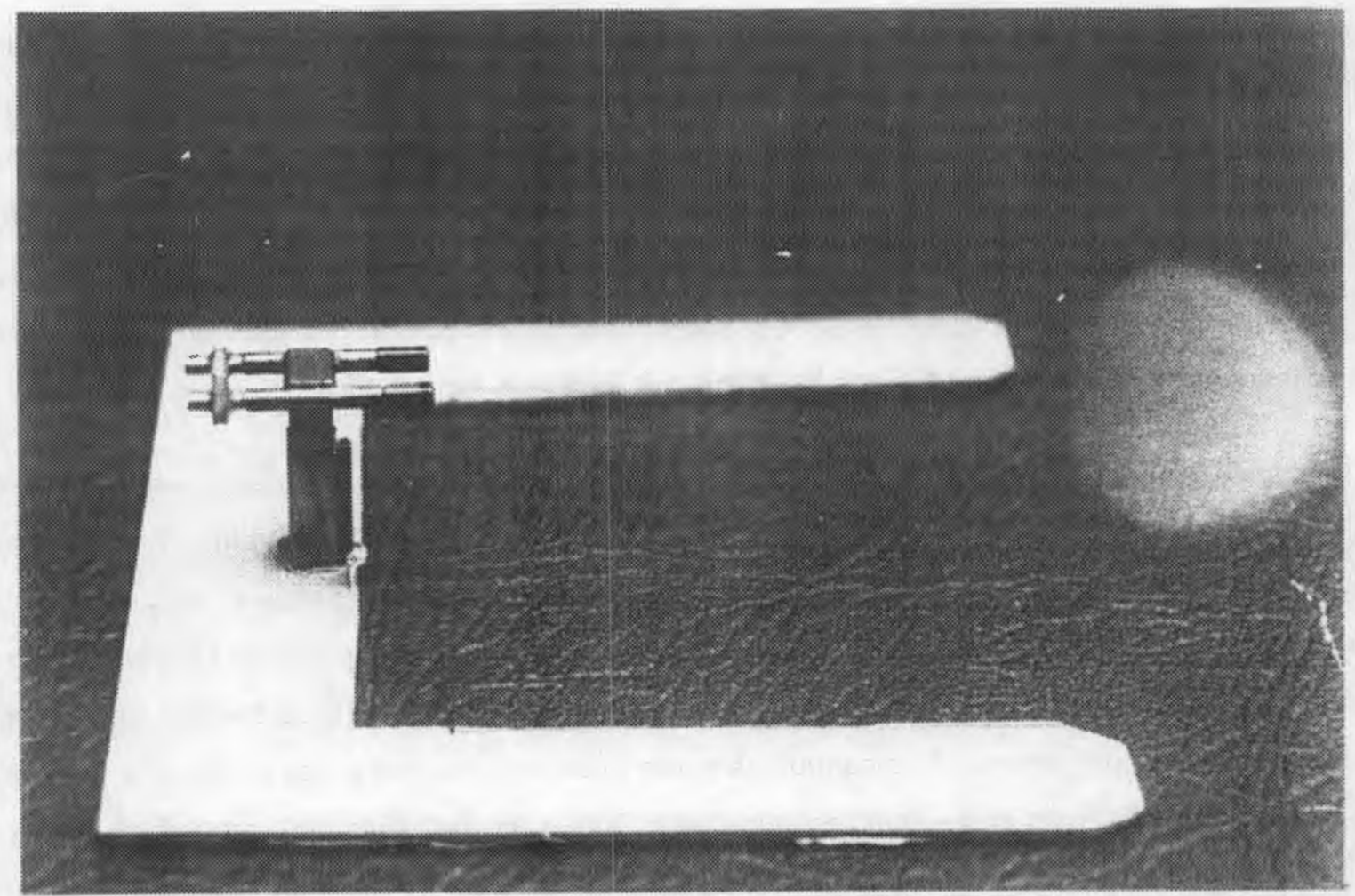

FIGURE B.4

The axially-rotatable stand. specially made for photographing corrosion-cast specimens.

They were then photographed, with surrounding side-lighting, through the microscope against a dark background, which was formed by the shadow in a deep hole in the background material (Fig B.5). 
Each vessel cast was rotated to show its widest and also its narrowest aspects for photographs. The resultant magnified prints (Fig B.6) could be measured easily and accurately by means of vernier calipers and then scaled back to life-size by means of the scale provided by the standard rod.

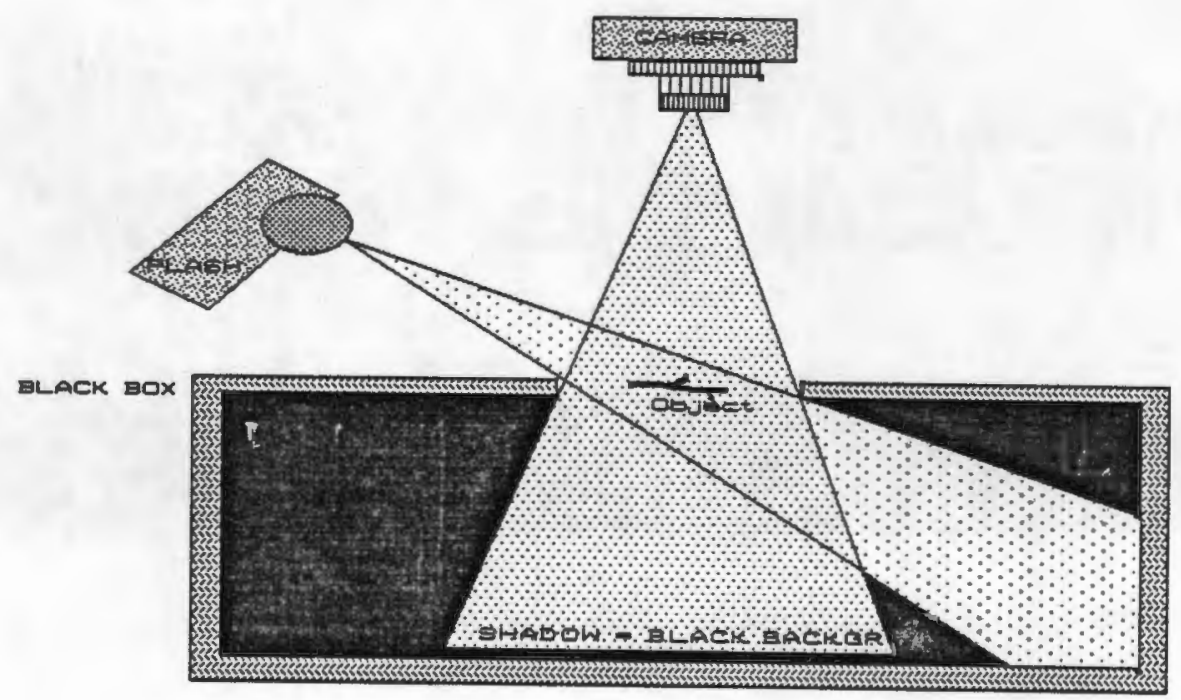

FIGURE B.5

A dark background which was formed by the shadow in a deep hole in the background material

We attempted to determine the shrinkage of the compound by making casts within plastic tubes of known internal diameter and allowing the casts to cure under the same physical conditions as those made in the blood vessels. This was done by placing the filled tubes in the abdominal cavity of the specimen animal during the polymerising process. The diameters of these casts were later compared with those of steel rods which fitted exactly into the lumina of the plastic tubes.

The ability of the compound to mirror surface markings was tested by making casts of the surfaces of two slightly different X-Ray films. A number of other persons were then asked to use the microscope to match cast to surface. 


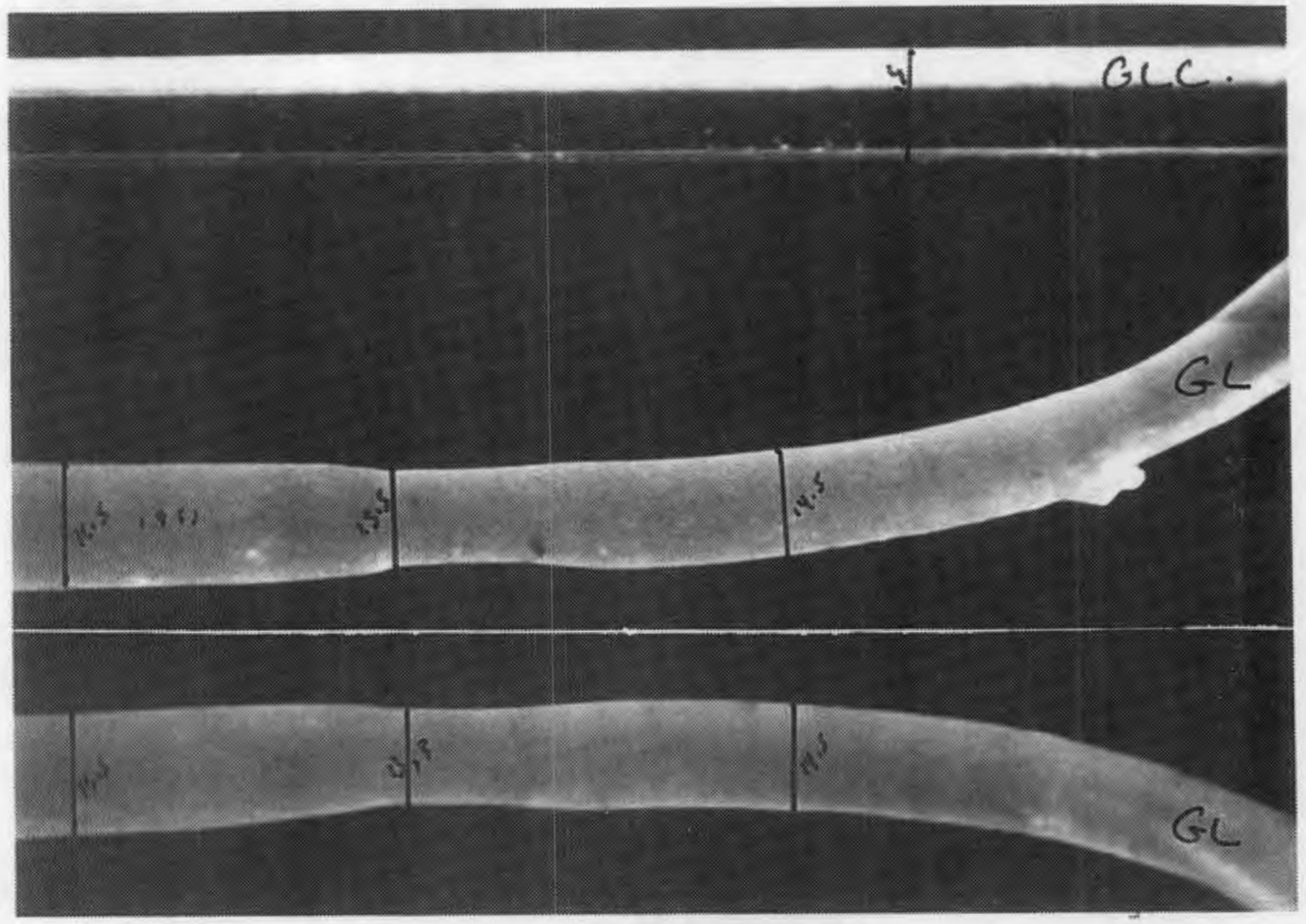

FIGURE B.6

Magnified images of the corrosion casts could be used for obtaining accurate measurements.

\section{RESULTS}

Using the methods described, we performed 32 corrosion cast procedures to examine 58 anastomoses. There were no cases in which we failed to obtain a cast of the desired anatomical area. In every cast it was possible to identify the anastomotic site. At each of the anastomotic sites it was possible to distinguish small irregularities in the "images" of the internal surfaces of the vessels. The three-dimensional demonstration of the shape of the vascular lumen was excellent, providing the possibility of detailed examination from all sides. Areas of stenosis or dilation could be clearly identified, measured and compared. The control casts, which had been made in plastic tubes under physical conditions identical to those of the vascular casts, were photographed beside their steel partners. The photographs were enlarged for measurement 
(Fig 8.7). At vessel diameters of $1 \mathrm{~mm}$ and less with which we were dealing, we could find no detectable shrinkage.

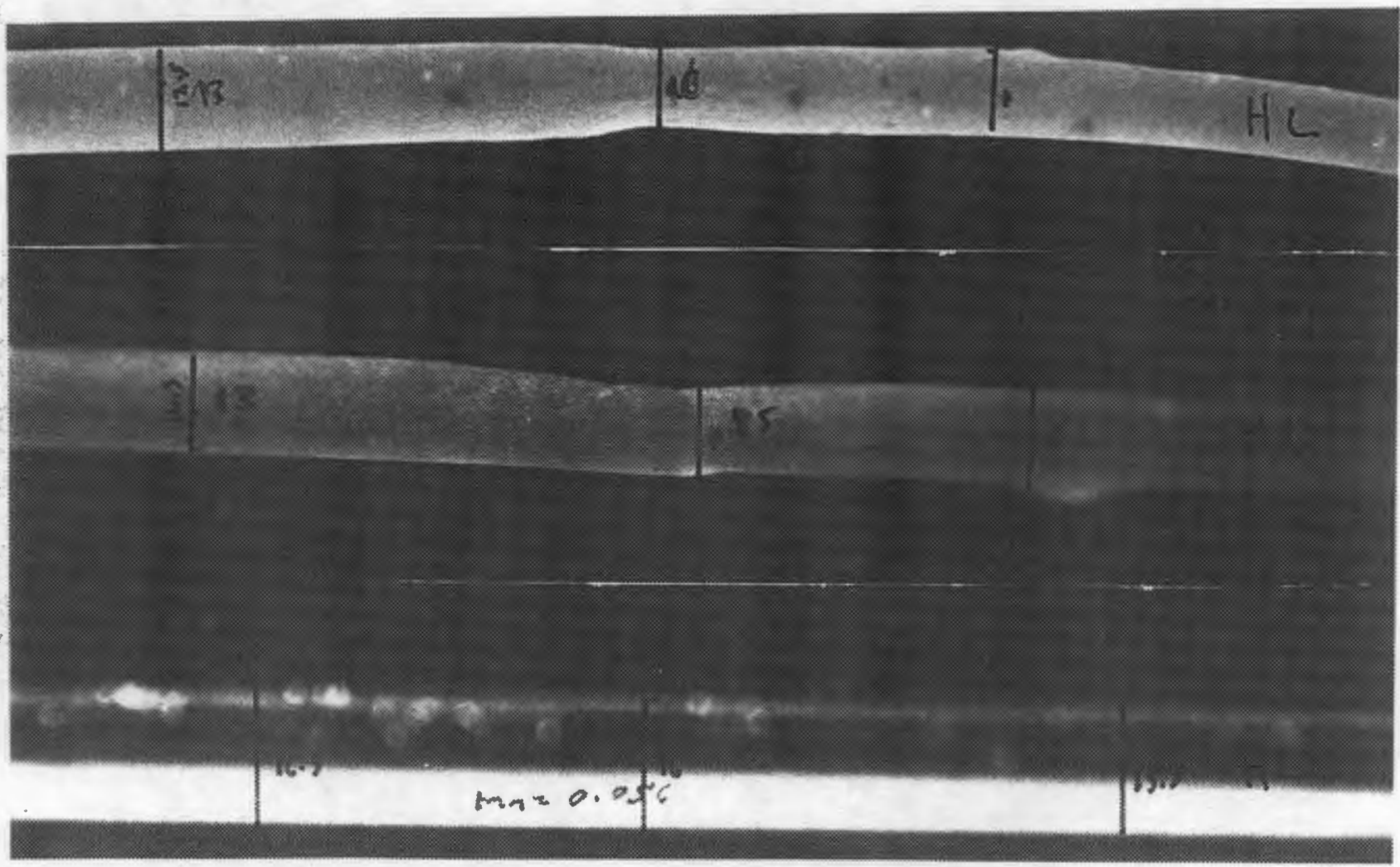

FIGURE 8.7

Measurements were taken trom photographic enlargements

In the surface reproduction test, where two surfaces of slightly differing microscopic texture had to be matched with their respective casts, there were no mistakes in 10 assessments by different individuals. The test surfaces were so similar that matching could only be performed at the highest magnifications of the operating microscope. 


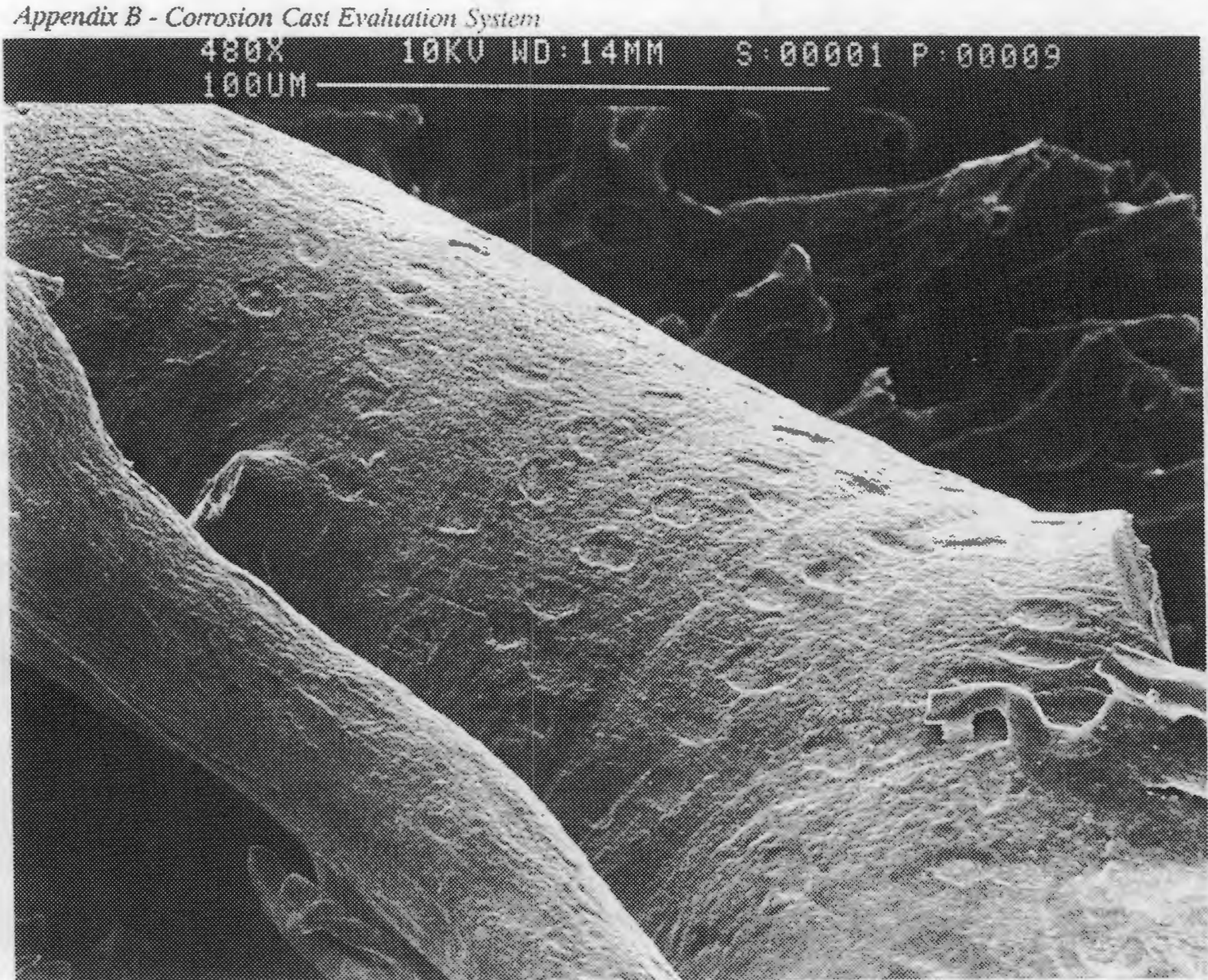

FIGURE B.8

A corrosion cast of a small vessel showing the depressions made by endothelial cell nuclei on the surface of the cast.

Scanning electron microscope (SEM) examination of the casts was subsequently performed and it was possible to distinguish quite clearly the impressions made by the nuclei of endothelial cells which had lined the vessels (Fig B.8).

\section{DISCUSSION}

Many workers have evaluated the morphology of microvascular anastomoses. The methods used have been:

1. direct examination $(1,78)$;

2. microscopic examination of histological sections of the vessel taken at the anastomotic site $(120,121,100,205.122)$; 


\section{SEM examination of suitably prepared vessel segments (109); and \\ 4. Radiographic techniques such as digital subtraction angiography (47).}

Radiographic techniques have a distinct advantage in that advances made in the experimental evaluation of anastomoses can rapidly be applied to the clinical situation. However, the interpretation of radiographic images of structures, or of contrast media within structures, requires a certain degree of inference and extrapolation which must be based on a knowledge of the actual appearance of the structure. An interesting aspect of the study of corrosion casts is the possibility of using a radio-opaque substance mixed with the resin. The radiographic appearance of the specimen may then later be correlated with the three-dimensional appearance of the resin cast after corrosion of the tissues has been effected.

The initial stages of the preparation for corrosion casting may be adapted for other investigative purposes such as tissue fixation for histological or electron microscopic investigation.

I abandoned the use of pressure-measuring equipment during the injection of resin, as it contributed nothing but excessive complication of the procedure. By observing the heart carefully during the injection and avoiding its distension a physiological pressure-control system is virtually being applied.

It is possible that the evaluation of corrosion casts may change some of the topographical concepts we have come to accept on the basis of histological studies. I believe that the distortion of structures by this corrosion-casting method is very much less than that caused by conventional histological preparation.

\section{SUMMARY}

I have described a simple and reliable method for producing corrosion endocasts of the vascular tree in small animals such as the rat and a method for employing these casts to evaluate experimental microvascular anastomoses. In my opinion the use of corrosion casts to perform physical measurements would best be applied to the comparative evaluation of pairs of anastomoses. Under identical physical conditions casts of two different vessels, produced from the same resin mix and injected at a single site, may confidently be compared. The casts which 
Appendix B - Corrosion Cast Evaluation System

are obtained, may be studied in many different ways and they will provide durable records of the surface morphology of the vascular lumina. 


\section{APPENDIX C}

THE CORROSION CAST EVALUATION OF

EXPERIMENTAL MICROVASCULAR ANASTOMOSES

IN RAT FEMORAL ARTERIES

\begin{tabular}{||c|c|c|c|c|c|c||}
\hline $\mathrm{n}=42$ & \multicolumn{3}{|c|}{ ETEA } & \multicolumn{3}{c|}{ SLEEVE } \\
\hline \hline DIAM. (mm) & PROX & ANAS & DIST & PROX & ANAS & DIST \\
\hline \hline MAXIMUM & 1.237 & 1.257 & 1.242 & 1.249 & 1.251 & 1.256 \\
MINIMUM & 0.715 & 0.698 & 0.718 & 0.716 & 0.694 & 0.719 \\
AVERAGE & 0.999 & 1.008 & 1.004 & 1.008 & 0.999 & 1.013 \\
STD.DEV & 0.168 & 0.172 & 0.169 & 0.166 & 0.168 & 0.166 \\
\hline \hline CSA (mm $\left.{ }^{2}\right)$ & & & & & & \\
\hline \hline MAXIMUM & 1.181 & 1.223 & 1.190 & 1.219 & 1.206 & 1.231 \\
MINIMUM & 0.409 & 0.390 & 0.413 & 0.407 & 0.383 & 0.411 \\
AVERAGE & 0.806 & 0.821 & 0.814 & 0.819 & 0.805 & 0.827 \\
STD.DEV & 0.258 & 0.267 & 0.261 & 0.258 & 0.259 & 0.260 \\
\hline
\end{tabular}

FIGURE 6.12

Analysis of results obtained from measurements of corrosion casts. 


\section{APPENDIX D}

\section{UNIVERSITY OF GÖTEBORG DEPARTMENT OF PLASTIC SURGERY}

SAHLGRENSKA SJUKHUSET 41345 GÖTEBORG SWEDEN tel. $031 / 410010$

March 2, 1987

Dr F J Duminy

Department of Microsurgery

Medical School

University of Cape Town

Observatory 7925

Cape Town

South Africa

Dear Dr Duminy:

Thank you for your letter of February 4. There is absolutely

\section{Body of letter memoved}

So in conclusion, thank you and I sincerely wish you all the luck for a successful dissertation and a happy future. I will look you up when ever (if ever) I come to your part of the world.

With my best personal regards,
Claes Lauritzen

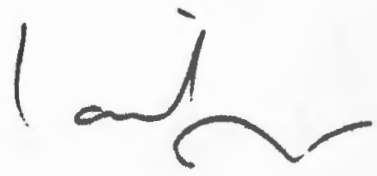

P.S. I forgot to say, I think your method is the ultimate sophistication of the sleeve. I would use it myself. 


\section{BIBLIOGRAPHY}

The more I read the more I meditate; and the more I acquire, the more I am enabled to affirm that I know nothing.

Voltaire (1764)

\section{$\underline{A}$}

\section{Acland R:}

Signs of patency in small vessel anastomosis.

Surgery 72: 744-748, 1972.

2. Acland R.D., Lubbers L.L., Grafton R.B. and Bensimon R.: Irrigating solutions for small vessel surgery - a histologic comparison. Plast. Reconstr. Surg. 65(4):460-465, 1980.

3. Acland R.D. and Trachtenberg L.:

The histopathology of small arteries following experimental microvascular anastomosis.

Plast. Reconstr. Surg. 59: 868-875, 1977.

4. Acland R.D.:

Photomicrography through the operating microscope.

Plast. Reconstr. Surg. 60(5):730-734, 1977.

\section{Acland R.D.:}

New instruments for microvascular surgery.

Brit. J. Surg. 59(3):181-184, 1972.

\section{Acland R.D.:}

A new needle for microvascular surgery.

Surgery. 71(1):130-131, 1972. 
7. Acland R.D.:

Microvascular anastomosis: A device for holding stay sutures and a new vascular Clamp.

Surgery. 75(2): 185-187, 1974.

8. Acland R.D.:

Thrombus formation in microvascular surgery: An experimental study of the effects of surgical trauma.

Surgery. 73(5):766-771, 1973.

\section{Acland R.D.:}

Notes on the handling of ultrafine suture material.

Surgery. $77(4): 507-511,1975$.

10. Acland R.D.:

Preventing thrombosis in microvascular surgery by the use of magnesium sulphate. Br. J. Plast. Surg. 25:292-299, 1972.

11. Alpert B.S., Buncke H.J. and Brownstein M.:

Replacement of damaged arteries and veins with vein grafts when replanting crushed, amputated fingers.

Plast. Reconstr. Surg. 61(1):17-22, 1978.

12. Asko-Seljavaara S., Pitknen J. and Sundell B.:

Microvascular free flaps in early reconstruction of burns in the hand and forearm.

Scand. J. Plast. Reconstr. Surg. 18:139-144, 1984.

\section{$\underline{B}$}

15. Ballantyne D.L., Reiffel R.S. and Harper A.D.:

Asystematic learning program for microvascular technique.

Plast. Reconstr. Surg. 65(1):80-82, 1980.

16. Batson O.V.

Corrosion specimens prepared with a new material.

Anat. Rec. 121: 425, 1955 (abstract)

17. Baudet J., Guimberteau J.C. and Nascimento E.:

Successful clinical transfer of two free thoracodorsal axillary flaps.

Plast. Reconstr. Surg. 58: 680-688, 1976 
18. Baumeister R.G., Siuda S., Bohmert H. and Moser E.:

A microsurgical method for the reconstruction of interrupted lymphatic pathways.

Scand. J. Plast. Reconstr. Surg. 20:141-146, 1986.

19. Bell L.D., Blair W.F., Pedersen D.R. and Gabel R.H.:

A Hemodynamic Evaluation of Cuffed Microarteriorhaphy.

Microsurgery 6:106-112, 1985.

\section{Booz Von K.H.}

Experimentelle Untersuchungen zum Problem der kontraktil aktiven V.portae des Meerschweinchens.

Anat. Anz. Bd. 113: 68-80, 1963.

21. Buncke H.J. and Blackfield H.M.:

The vasoplegic effects of chlorpromazine.

Plast. Reconstr. Surg. 31:353-362, 1963.

22. Buncke H.J. and Schulz W.P.:

Experimental digital amputation and reimplantation.

Plast. Reconstr. Surg. 36: 62-70, 1965. .

23. Buncke HJ., Buncke C.M., and Schulz W.P.:

Immediate Nicoladoni procedure in the rhesus monkey, or hallux-to-hand

transplantation, utilizing microminiature vascular anastomoses.

Br. J. Plast. Surg. 19:332-337, 1966.

24. Buncke H.J. and Schulz W.P.:

Total ear replantation in the rabbit using microminiature vascular anastomoses.

Br. J. Plast. Surg. 19: 15-22, 1966.

\section{Buncke H.J.}

Toe digital transfer.

Clin. Plast. Surg. 3: 49-54, 1976.

26. Buncke H.J., Furnas D.W., Gordon L. and Achauer B.M.:

Free osteocutaneous fiap from a rib to the tibia.

Plast. Reconstr. Surg. 59(6):799-805, 1977.

\section{Buncke H.J., Furnas D.W. [Editors] :}

Symposium on clinical frontiers in reconstructive microsurgery. [Proceedings of the Symposium of the Plastic Surgery Educational Foundation of the American Society of Plastic and Reconstructive Surgeons Inc., held in Anaheim, California, June 2528, 1981] The C.V.Mosby Company, St.Louis Toronto 1984 
- Bibliography -

28. Buncke H.J., McLean D.H., George P.T., Creech B.J., Chater N.L. and Commons G.W.: Thumb replacement: Great toe transplantation by microvascular anastomosis. Br. J. Plast. Surg. 26:194-201, 1973.

\section{$\underline{\mathbf{C}}$}

29. Caduff J.H., Fischer L.C. and Burri P.H.:

Scanning electron microscope study of the developing microvasculature in the postnatal rat lung.

Anat. Record 216: 154-164, 1986.

30. Caffee H.H. and Ward D.:

Bipolar coagulation in microvascular surgery.

Plast. Reconstr. Surg. 78(3):374-377, 1986.

31. Carrel A. and Guthrie C.C.:

Anastomosis of blood vessels by the patch method and transplantation of the kidney.

JAM A 47: 1648, 1906.

32. Carrel A. and Guthrie C.C.:

Complete amputation of the thigh with replantation.

Am. J. Med. Sci. 131: 297-301, 1906.

33. Carrel A. :

Ultimate results of aortic transplantations.

J. Exp. Med 15: 389-392, 1912.

\section{Carrel A.}

The surgery of blood vessels.

Bull.Johns Hopkins Hosp. 18: 18-28, 1907.

35. Carrel A.

La technique opratoire des anastomoses vasculaires et la transplantation des viscres.

Lyon Med. 98: 859-864, 1902. The operative technique of vascular anastomosis and transplantation of organs. 
(English translation Clin. Orth. Vol 29, 1963)

36. Chase R.A.:

Expanded clinical and research uses of composite tissue transfers on isolated vascular pedicles.

Am. J. Surg. 114: 222-224, 1967.

37. Chen C.W., Chien Y.C., Pao Y.S. and Lin C. T.:

Reattachment of traumatic amputations: A summing up of experience.

Chin. Med. J. 5: 392, 1967.

38. Chen C.W.:

Replantation of severed limbs and fingers.

Chin. Med. J. 1: 3, $1973 .$.

39. Cheng G., Pan D., Qu Z., Lin B. and Yang Z.:

Replantation of avulsively amputated thumb: a report of 15 cases.

Ann Plast Surg. 15(6): 474-480, 1985.

40. Chignier E. and Eloy R.

Adventitial resection of small artery provokes endothelial loss and intimal hyperplasia.

Surg., Gyne. \& Obstet. 163:327-334, 1986.

41. Choi H.K., Stowe N. and Novic A.C.:

Comparison of end-to-end and telescoped arterial anastomoses in renal transplantation in rats.

J. Microsurg. 3: 85-88, 1982.

42. Chow S.P., Yang K.F., Luk D.K. and So Y.C.:

Experimental microarterial grafts: The use of freeze-dried human placental vessels as heterografts.

Plast. Reconstr. Surg. 75(5):703-706, 1985.

43. Christie B.A., Ketharanathan V. and Perloff LJ.:

Minute vascular replacements.

Arch Surg. 117:1290-1294, 1982.

44. Clark E.B., Rooney P.R., Martini D.R, and Rosenquist G.C.:

Plastic casts of Embryonic respiratory and cardiovascular system: a technique.

Teratology 19: 357-360, 1979.

45. Cobbett J.R.

Small vessel anastomosis: a comparison of suture techniques.

Br. J. Plast. Surg. 22: 16-20, 1967. 
46. Colen J.W., Gonzales F.P. and Buncke H.J.:

The relationship between the number of sutures and the strength of microvascular anastomoses.

Plast. Reconstr. Surg. 64: 325-329, 1979.

47. Cook A.F., Grossman J.A.I., Herpy E.S. and Dinner M.I.:

A Method for quantitative analysis of lumen changes in microvascular anastomoses. J. Microsurg. 3:54-55, 1981.

48. Crafoord C. and Nylin, G.:

Congenital coarctation of the aorta and its surgical treatment.

J. Thorac. Surg. 14: 347-361, 1945.

49. Cuadros C.L.:

One hundred percent patency of one -millimetre polytetrafluoroethylene (Gore-tex) grafts in the carotid arteries of rats.

Microsurgery 5:000-000, 1983. (copy of galley proof)

$\underline{\mathbf{D}}$

52. Daniel R.K. and Taylor, G.I.:

Distant transfer of an island flap by microvascular anastomosis.

Plast. Reconstr. Surg. 52: 111-116, 1973.

53. Daniel R.K., Terzis J. and Midgely R.D.:

Restoration of sensation to an anasthetic hand by a free neurovascular flap from the foot.

Plast. Reconstr. Surg. 57: 275-280, 1976.

\section{Davies D.M.:}

A world survey of anticoagulation practice in clinical microvascular surgery.

Br. J. Plast. Surg. 35: 96-99, 1982.

55. Dollinger R.K. and Armstrong P.B. :

Scanning electron microscopy of injection replicas of the chick embryo circulatory system.

J. Microsc. 102: 179-186, 1974.

56. Dorrance G.M.:

An experimental study of suture of arteries with a description of a new suture.

Ann. Surg. 44:409-424, 1906. 
57. Dorfler J.:

Uber Arteriennaht.

Beitrage z. Klin. Chir. 25:781, 1899.

\section{$\underline{E}$}

59. Eisenhardt H.J., Hennecken H., Klein P.J. et al:

Experiences with different techniques of microvascular anastomosis.

J. Microsurg. 1:341-350, 1980.

60. Essien E.M., Cazenave J.P., Moore S. and Mustard J.F.:

Effect of heparin and thrombin on platelet adherence to the surface of rabbit aorta. Thrombosis Research 13: 69-74, 1978. .

63. Floyd H.D, Kerstein M.D.:

Successful vascular reconstruction, determinants of disability.

American Surgeon 52:91-92, 1986.

64. Foucher G., Merle M., Maneaud M. and Michon J.:

Microsurgical free partial toe transfer in hand reconstruction: A report of 12 cases.

Plast. Reconstr. Surg. 65(5):616-626, 1980.

65. Fronticelli C.M., Bargoni A., Discalzo L. et al:

Telescopic anastomosis of the common bile duct in the rat.

Microsurg. 4: 115-119, 1983.

$\underline{\mathbf{G}}$

68. Geter R.K., Winters R.R.W., Puckett C.L.:

Resolution of experimental microvascular spasm and improvement in anastomotic patency by direct topical agent application.

Plast. Reconstr. Surg. 77(1): 105-115, 1986. 
69. Glimelius B., Busch C. and Hook M.:

Binding of heparin on the surface of cultured human endothelial cells.

Thrombosis Research 12:773-779, 1978. .

70. Godina M.:

Arterial autografts in microvascular surgery.

Plast. Reconstr. Surg. 78:293-294, 1986.

71. Guthrie C.C.:

"Blood Vessel Surgery and its Applications"

Edward Arnold, London 1912.

(Reprinted by University of Pittsburgh Press 1959).

\section{H}

72. Haimovici $\mathrm{H}_{\text {.: }}$

Matas lecture: The early pioneers in vascular surgery and their legacy.

J. Cardiovas. Surg. 25: 275-286, 1984.

73. Hall R.F. and Watt D.H.:

Osseous changes due to $a$ false aneurysm of the proper digital artery: A case report.

J. Hand Surg. 11A(3):440-442, 1986.

74. Harashina $T$. and Irigaray A.

Expansion of smaller vessel diameter by fish-mouth incision in microvascular anastomosis with marked size discrepancy.

Plast. Reconstr. Surg. 65(4):502-503, 1980.

75. Harii $\mathbf{K}$, Ohmori $\mathbf{K}$, and Ohmori S.:

Free deltopectoral skin flaps.

Br. J. Plast. Surg. 27:231-239, 1974.

76. Harris G.D., Finseth F. and Buncke H.J.:

Posterior-wall-first microvascular anastomotic technique.

Br. J. Plast. Surg. 34: 47-49, 1981.

77. Harris G.D., Finseth F. and Buncke H.J.:

The microvascular anastomotic autogenous cuff.

Br. J. Plast. Surg. 34: 50-52, 1981. 
78. Hayhurst J.W. and O'Brien B.McC.:

An experimental study of microvascular technique, patency rates and related factors.

Br. J. Plast. Surg. 28: 128-131, 1975.

79. Hedberg S.E.:

Suture anastomosis of small vessel following relief of spasm by hydrostatic pressure dilation.

Ann. Surg. 155: 51-59, 1965.

80. Heden P.G., Hamilton R., Arnander C. and Jurrel G.:

Laser Doppler surveillance of the circulation of free flaps and replanted digits.

Microsurgery 6(1):11-19, 1985.

81. Hess F.:

History of (Micro)Vascular Surgery and the Development of Small-Caliber Blood

Vessel Prostheses (With some notes on patency rates and re-endothelialization).

Microsurgery 6(2):59-69, 1985.

82. Hiebert L.M. and Jaques L.B.:

The observation of heparin on endothelium after injection.

Thrombosis Research 8: 195-199, 1976.

83. Holmin T., Buchholtz B., Flati G. et al :

A simplified method for total arterialization of the liver in rats.

Microsurg. 4: 57-60, 1983.

84. Huang G-K.,Hu R-Q., Miao H., Yin Z-Y., Lan T-D. and Pan G-P.:

Microvascular free transfer of iliac bone based on the deep superior branches of the superior gluteal vessels.

Plast. Reconstr. Surg. 75(1):68-74, 1985.

85. Huang C.-D., Chow S.P. and Chan C.W.:

Experience with anastomoses of arteries approximately $0.2 \mathrm{~mm}$ in external diameter.

Plast. Reconstr. Surg. 69(2): 299-305, 1982.

86. Hubbard J.C.:

Arteriovenous anastomosis.

Ann. Surg. 44:559-562, 1906.

87. Hyland W.T., Botens S.R. and Minasi J.S.:

A re-appraisal and modification of the Lauritzen technique of microvascular anastomoses.

Br. J. Plast. Surg. 34: 451-453, 1981. 
- Bibliography -

88. Henderson H.P. and Sully L.

Problems encountered in the replantation of an avulsed scalp: use of leeches in replantation surgery.

Br. J. Plast. Surg. 36:219, 1983.

90. Ikuta Y, Kubo T. and Tsuge K.:

Free muscle transplantation by microsurgical technique to treat severe Volkmann's contracture.

Plast. Reconstr. Surg. 58: 407-411, 1976.

\section{$\underline{\mathbf{J}}$}

93. Jacobson J.H.:

Microsurgical technique in repair of the traumatized extremity.

Clin. Orthop. 29: 132-145, 1963.

\section{Jacobson J.H. and Suarez E.L.:}

Microsurgery in anastomosis of small vessels.

Surg. Forum 11: 243-245, 1960.

\section{$\underline{\mathbf{K}}$}

97. Kamada N. and Calne R.Y.:

Orthotopic liver transplantation in the rat.

Transplantation 28(i):47-50, 1979.

98. Kleinert H.E. and Kasdan M.L.:

Salvage of devascularized upper extremities Including studies on small vessel anastomosis.

Clin. Orthop. 29: 29-38, 1963.

99. Komatsu S. and Tamai S.:

Successful replantation of a completely cut-off thumb.

Plast. Reconstr. Surg. 42: 374-377, 1968. 
100. Krag C., Holck S., De Rose G., Lyczakowski T. and Freeman C.R.: Healing of microvascular anastomoses.

Scand. J. Plast. Reconstr. Surg. 16:267-274, 1982.

101. Krag C. and Lavendt E.:

Late patency of clinical microvascular anastomoses to free composite tissue transplants.I. Angiographical Aspects.

Scand. J. Plast Reconstr Surg. 19: 65-72, 1985.

102. Krag C., Hesselfeldt-Nielsen J. and Gothgen I.:

Late patency of clinical microvascular anastomoses to free composite tissue transplants. II. Hemodynamical Aspects.

Scand. J. Plast Reconstr Surg. 19: 73-79, 1985.

103. Krag C:

Experience with transplantation of composite tissues by means of microsurgical vascular anastomoses. (I Indications, Techniques and Early results).

Scand. J. Plast. Reconstr. Surg. 19:135-155, 1985.

104. Krag C:

Experience with transplantation of composite tissues by means of microsurgical vascular anastomoses. (II Late results and comments).

Scand. J. Plast. Reconstr. Surg. 19:157-173, 1985.

105. Krizek T.J., Tani R., Desprez J.D. and Kiehn C.L.:

Experimental transplantation of composite grafts by microsurgical vascular techniques.

Plast. Reconstr. Surg. 36: 538-546, 1965.

$\underline{\mathbf{L}}$

107. Lauritzen Claes :

The Microvascular Sleeve Anastomosis (Thesis).

The Laboratory of Experimental Biology, Dept. of Anatomy and Dept. of Plastic Surgery,

University of Gteborg, Sweden. Gteborg 1979.

108. Lauritzen $C$. and Bagge U.:

A technical and biomechanical comparison between two types of microvascular anastomoses: an experimental study in rats.

Scand. J. Plast. Reconstr. Surg. 13: 417-421, 1979. 
109. Lauritzen C. and Hansson H.A.:

Microvascular repair after the sleeve anastomosis: an ultrastructural study in the rat femoral vessels.

Scand. J. Plast. Reconstr. Surg. 14: 65-70, 1980.

110. Lauritzen C., Fogdestam I., Hamilton R. and Johannson B.:

The sleeve anastomosis in clinical microsurgery: case report.

Scand. J. Plast. Reconstr. Surg. 13: 477-479, 1979

111. Lauritzen C., Johannson B.R. and Eriksson E.:

Long-term study of the sleeve anastomosis: an experimental study in the rabbit renal artery.

Scand. J. Plast. Reconstr. Surg. 14: 165-169, 1980.

112. Lauritzen C.:

A new and easier way to anastomose microvessels.

Scand. J. Plast. Reconstr. Surg. 12: 291-294, 1978.

113. Lauritzen $\mathrm{C}$.:

Discussion of: A histologic comparison of experimental microarterial end-in-end (sleeve) and end-to-end anastomoses. (Wieslander J.B. and Rausing A.)

Plast. Reconstr. Surg. 73: 286-287, 1984.

114. Lauritzen C.:

Sleeve anastomosis [Letter]

Plast. Reconstr. Surg. : 424,Sept 1983.

115. Lauritzen C.:

A presentation of the sleeve anastomosis.

Dept. of Plastic Surgery, Sahlgrenska sjukhuset, University of Gteborg, Gteborg, Sweden. 1979, 49-51.

116. Lauritzen C.G.:

The sleeve anastomosis revisited.

Ann. Plast. Surg. 13(2):145-149, 1984.

117. Lauritzen $C_{\text {: }}$

Experimental studies on absorbable vascular grafts for microsurgery.

Scand. J. Plast. Reconstr. Surg. 17:133-135, 1983.

118. Lee S., Frank D.H. and Choi S.Y.:

Historical review of small and microvascular vessel surgery.

Ann. Plast. Surg. 11: 53-62, 1983. 
119. Lee S.,(lee2) Charters A.C., Chandler J.G. Orloff M. J.: A technique for orthotopic liver transplantation in the rat. Transplantation 16(6): 664-669, 1973

120. Lidman D. and Daniel R.K.:

The normal healing process of microvascular anastomoses.

Scand. J. Plast. Reconstr. Surg. 15:103-110, 1981.

121. Lidman D. and Daniel R.K.

Evaluation of clinical microvascular anastomoses - Reasons for failure.

Ann Plast Surg. 6(3):215-223, 1981.

122. Lidman D., Lyczakowski T. and Daniel R.K.

The morphology and patency of arterial and venous microvascular anastomoses throughout the first post-operative year.

Scand. J. Plast. Reconstr. Surg. 18:187-192, 1984.

\section{$\underline{\mathbf{M}}$}

124. Malt R.A. and McKhann C.F.:

Replantation of severed arms.

J.A.M.A. 189: 716-722, 1964.

125. Man D. and Acland R.D.:

Continuous suture techniques in microvascular end-to-end anastomosis.

J.Microsurg. 2:238-243, 1981.

126. Mann F.C., Priestley J.T., Markowitz J. et al:

Transplantation of the intact mammalian heart.

Arch. Surg. 26: 219-227, 1933.

127. Margic K:

Early changes in dissected small vessels: Experimental study on rat arteries and veins.

Plast. Reconstr. Surg. 75(3):375-382, 1985.

128. Mauser R. and Mauser R.:

$\mathrm{Hf}$-sonographische analyse des postoperativen blutstromungsverhaltens nech mikrovaskularen anastomosen.

Handchir. Mikrochir. Plast. Chir 17(5):251-254, 1985. 
129. Maxwell G.P., Szabo Z. and Buncke H.J.:

Aneurysms after microvascular anastomosis, Incidence and pathogenesis in experimental animals.

Plast. Reconstr. Surg. 63(6):824-829, 1979.

130. McLean D.H. and Buncke H.J.:

Autotransplantation of omentum to a large scalp defect with microsurgical revascularization.

Plast. Reconstr. Surg. 49: 268-273, 1972.

131. Meier W.E.:

Zum anastomosenproblem kleiner Arterien.

Helv. Chir. Acta 45: 167-170, 1978.

132. Merkel J.C., Zook E.G. and Russel R.C.:

Cuffing techniques in microarterial surgery.

J. Hand Surg. 9A(1):76-82, 1984.

133. Meyer V.E., Smahel J. and Donski P.:

Microvascular anastomosis using the telescope principle: experimental study.

Int. J. Microsurg. 2: 81-86, 1980.

134. Minderjahn A and Dahm H.H.:

Scanning electron microscope observations of microvascular anastomosis in the rat carotid artery.

J. Max.fac. Surg. 7:225-234, 1979.

135. Murphy J.B:

Resection of arteries and veins injured in continuity, end-to-end suture:

experimental and clinical research.

Med. Record 51: 73-88, 1897.

136. Melka J., Charbonneau R. and Bosse J.-P.:

Experimental evaluation of microarterial grafts in rats and rabbits : Long-term histological studies.

Plast. Reconstr. Surg. 63(2):245-249, 1979.

\section{$\underline{\mathbf{N}}$}

137. Nakayama Y., Soeda S., lino T. and Uchida A.:

Is the sleeve anastomosis a risky technique?

Br. J. Plast. Surg. 40:288-294, 1987 
138. Nakayama $K$., Tamiya $T$., Yamamoto $K$. and Akimoto $S$.:

A simple new apparatus for small vessel anastomosis.

Surgery 52: 918-931, 1962.

139. Nakayama $K$, Yamamoto $K$. and Makino $H$.:

A new vascular anastomosing instrument and its clinical application.

Clin. Orthop. 29: 123-131, 1963.

140. Narat J.K., Loef J.A. and Narat M.:

On the preparation of multicolored corrosion specimens.

Anat. Record 64(2):155-159

141. Nieuborgh L:

Assessing the patency of microvascular anastomoses.

Br. J. Plast. Surg. 32:151-152, 1979.

142. Nordgren S. and Cohen Z:

Arteriovenous shunt using the sleeve technique in the dog.

Surg. Gynecol. Obstet. 158: 231-232, 1984.

143. Nylen C.O.:

The otomicroscope and microsurgery, 1921-1971.

Acta Otolaryngol. 73: 453-454, 1972.

\section{$\underline{0}$}

146. O'Brien B.McC., MacLeod A.M., Hayhurst J.W. and Morrison W.A.

Successful transfer of a large island flap from the groin to the foot by microvascular anastomoses.

Plast. Reconstr. Surg. 52:271-278, 1973.

147. O'Brien B.McC.:

Microvascular Reconstructive Surgery.

Churchill Livingstone, Edinburgh, London, New York 1977.

148. O'Brien C.J., Wilson E.A., Velkou D., Harris J.P. and May J.:

Experimental microvascular Polytetraflouroethylene grafts: 6-month patency.

Plast. Reconstr. Surg. 76(5):748-752, 1985. 
149. Pagnanelli D.M., Pait T.G., Rizzoli H.V. and Kobrine A.I.:

Scanning electron micrographic study of vascular lesions caused by microvascular needles and suture.

J. Neurosurg. 53: 32-36, 1980.

150. Pennington D.G., Lai M.F. and Pelly A.D.:

Successful replantation of a completely avulsed ear by microvascular anastomosis. Plast. Reconstr. Surg. 65(6):820-823, 1980.

151. Peters C.R., McKee D.M. and Berry B.E.: Pharyngoesophogeal reconstruction with revascularised jejunal transplants. Am. J. Surg. 121: 675-678, 1971.

152. Petry J.J., French T.S. and Wortham K.A.:

The Effect of the "Patency test" on arterial endothelial surface.

Plast. Reconstr. Surg.77(6):960-963, 1986.

153. Pupini G.:

Contribution to experimental blood vessel anastomosis.

Clin Chirurgica. 35: 1165, 1932.

154. Payr E.

Beitrage zur technik der blutgefasse und nervennaht nebst mittheilungen uber die vervendung eines resorbirbaren metalles in der chirurgie.

Arch. Klin Chir. 62:67, 1900. (Eng Abstr)

\section{Q}

156. Quillen C.G., Shearin J.C. and Georgiade N.G.:

Use of the latissimus dorsi myocutaneous island flap for reconstruction of the head and neck area.

Plast. Reconstr. Surg. 62: 113-117, 1978. 
159. Rao V.K., Morrison W.A., Angus J.A. and O'Brien B.McC.:

Comparison of vascular hemodynamics in experimental models of microvascular anastomoses.

Plast. Reconstr. Surg. 71(2):241-247, 1983.

160. Rao V.K., Nightingale G. and O'Brien B.McC.:

Scanning electron microscope study of microvenous grafts to artery.

Plast. Reconstr. Surg. 71(1):98-106, 1983.

161. Rich N.M. and Spencer F.C.:

Vascular Trauma.

W.B. Saunders Co. Philadelphia. 1978.

162. Russell R.C., Van Beek A.L., Zook E.G. and Jacobs S.C.:

The growth potential of small arteries repaired by microsurgical technique.

Ann Plast Surg. 17(1):34-38, 1986.

164. Sauvage L.R. and Wood S.J.:

Vascular repair in the traumatised extremity.

Clin. Orthop. 29: 72-82, 1963.

165. Sawyer P.N. and Pate J.W.:

Bio-electric phenomena as an etiologic factor in intravascular thrombosis.

Am. J. Physiol. 175:103-107, 1953.

166. Sawyer P.N., Pate J.W. and Weldon C.S.:

Relations of abnormal and injury electric potential differences to intravascular thrombosis.

Am. J. Physiol. 175:108-112, 1953.

167. Schenck R.R., Weinrib H.P. and Labanauskas I.G.:

The external ring technique for microvascular anastomosis.

J. Hand Surg. 8: 105-108, 1983.

168. Schneider P.R., Pribaz J. and Russell R.C.:

Microvenous graft length determination for arterial repair.

Ann Plast Surg. 17(4):292-298, 1986. 
169. Seidenberg B., Hurwitt E.S. and Carton C.A.:

The technique of anastomosing small arteries.

Surg. Gynecol. Obstet. 106: 743-746, 1958.

170. Serafin $D_{\text {.: }}$

Microsurgery: past, present and future.

Plast. Reconstr. Surg. 66(5): 781-785, 1980.

171. Shaffer J.W., Kling T.F., Wilber G., Field G.A. and Williams H.J.

Patency of anastomosed arteries and veins subjected to reapplication of microvascular clamps.

J. Hand Surg. 9A(1): 73-75, 1984.

172. Silverberg B., Banis J.B., Verdi G.D., Acland R.D.:

Microvascular reconstruction after electrical and deep thermal injury.

J. Trauma 26(2):128-134, 1986.

173. Sinclair S.:

The importance of topical heparin in microvascular anastomoses: a study in the rat.

Br. J. Plast. Surg. 33: 422-426, 1980.

174. Smith A.R., Van Alphen B., Faithfull N.S. and Fennema M.:

Limb preservation in replantation surgery.

Plast. Reconstr. Surg. 75(2):227-237, 1985.

175. Snyder C.C. and Knowles R.P.:

Autoplantation of extremities.

Clin. Orthop. 29: 113-121, 1963.

176. Stamatopoulos C., Biemer E., Stock W. and Zechner W. :

Microvascular anastomosis by invagination: an experimental study.

J. Cardiovas. Surg. 23: 130-134, 1982.

177. Strauch B. and Murray D.E.:

Transfer of composite graft with immediate suture anastomosis of its vascular pedicle measuring less than $1 \mathrm{~mm}$ in external diameter using microsurgical techniques.

Plast. Reconstr. Surg. 40: 325-329, 1967.

178. Sully L., Nightingale M.G., O'Brien B.McC. et al:

An experimental study of the sleeve technique in microarterial anastomoses.

Plast. Reconstr. Surg. 70: 186-192, 1982. 


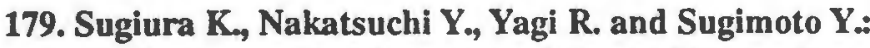

A new method for venous interposition grafts using fibrin glue.

Microsurgery 6:125-128, 1985.

\section{Sykes P.J.:}

Application of microvascular surgery to plastic surgery

J. Royal Soc. Med. 73: 55-59, 1980.

182. Tajima S.:

Trap-door technique in the background for holding stay sutures in the microvascular anastomosis.

Plast. Reconstr. Surg. 66 (3): 463-465, 1980

183. Takayanagi S. and Ota C.:

Influence of dryness of the vessel on its patency.

Ann Plast Surg. 12:525-527, 1984.

184. Taylor G.I., Miller G.D.H. and Ham F J:

The free vascularized bone graft: A clinical extension of microvascular techniques.

Plast. Reconstr. Surg. 55: 533-544, 1975

185. Thal A, Perry J.F., Miller F.A. et al:

Direct suture anastomosis of the coronary arteries.

Surgery 40: 1023-1029, 1956.

186. Thiede A, Lutjohann $\mathrm{K}$. Beck $\mathrm{C}$, et al :

Absorbable and nonabsorbable suture microsurgery: standardized comparable studies in rats.

J. Microsurg. 1: 216, 1979.

187. Thoma A.:

Storage of a free forearm flap for 55 hours.

Plast. Reconstr. Surg. 78(1):91-93, 1986.

188. Thurston J.B., Buncke H.J., Chater N.L. and Weinstein P.R.:

A scanning electron microscope study of micro-arterial damage and repair Plast. Reconstr. Surg. 57: 197-203, 1976. 
189. Tizian C.:

A new microvascular substitute (inside diameter $1 \mathrm{~mm}$ ): Evaluation and early patency rates.

Angiology 31(12):801-819, 1980.

190. Torrance G.:

Suture of the Brachial artery.

Ann. Surg. 40:107-108, 1904.

195. Vale B.H., Frenkel A., Trenka-Benthin S. and Matlaga B.F.:

Microsurgical anastomosis of rat carotid arteries with the $\mathrm{CO} 2$ laser.

Plast. Reconstr. Surg. 77(5): 759-766, 1986.

196. Van Gelder P.A. and Klopper P.J.:

Healing of microvascular arterial anastomosis as seen on corrosion casts by scanning electron microscopy.

Plast. Reconstr. Surg. 64: 59-64, 1979.

197. Vilkki S.K.

Microvascular sleeve anastomosis in clinical replantation.

Scand J Plast Reconstr Surg (Suppl) 19:71, 1982.

198. Van Beek A.L., Wavak P.L. and Zook E.G.:

Microvascular surgery in young children.

Plast.Reconstr. Surg. 63(4):457-462, 1979.

\section{$\underline{\mathbf{W}}$}

201. Watters W.B. and Buck R.C. :

An improved simple method of specimen preparation for replicas or scanning electron microscopy.

J. Microsc. 94: 185, 1971.

\section{Watts S.H.:}

The suture of blood vessels. Implantation and transplantation of vessels and organs. An historical and experimental study.

Johns Hopkins Hosp. Bull. 18: 153-179, 1907. 
203. Wieslander J.B., Mecklenburg C.V. and Aberg M.:

Endothelialization following end-to-end and end-in-end (sleeve) microarterial anastomoses.

Scand J Plast Reconstr Surg. 18:193-199, 1984.

204. Wieslander J.B. and Aberg M.:

Blood flow in small arteries after end-to-end and end-in-end anastomoses: an experimental quantitative comparison.

J. Microsurg. 2: 121-125, 1980.

205. Wieslander J.B. and Aberg M.:

Stenosis following end-in-end microarterial anastomosis: an angiographic comparison with the end-to-end technique.

J. Microsurg. 3:151-155, 1982.

206. Wieslander J.B. and Rausing A. :

A histologic comparison of experimental microarterial end-in-end (sleeve) and endto-end anastomoses.

Plast. Reconstr. Surg. 73: 279-285, 1984.

207. Wieslander J.B., Aberg M. and Dougan P.:

Accumulation of isotope labelled platelets in small arteries after end-to-end and endin-end anastomoses in the rabbit.

Br. J. Plast. Surg. 35: 158-162, 1982.

208. Williamson C.S. and Mann F.C.:

Functional survival of autogenous and homogenous transplants of blood vessels.

An experimental study.

Arch Surg. 54: 529-540, 1947.

\section{ADDENDA}

209. Cobbett J.R.

Free Digital Transfer (Report of a case of transfer of a Great Toe to replace an amputated thumb)

J.Bone and Joint Surgery 51B: 677-679, 1969

210. Duminy Francois J.

A new microvascular Sleeve anastomosis

J. Surgical Research 46: 189 - 194, 1989 


\section{- Bibliography -}

211. De Bakey Col. M.E. and Simeone Col. F.E.:

Battle injuries of the arteries in World War II

Ann. Surg. 123:534-573, 1946

212. Yasargil M.G.:

Experimental small vessel surgery in the dog including patching and grafting of cerebral vessels and the formation of functional extracranial shunts. C.V.Mosby \& Co St Louis 1967 pp 87-126 